



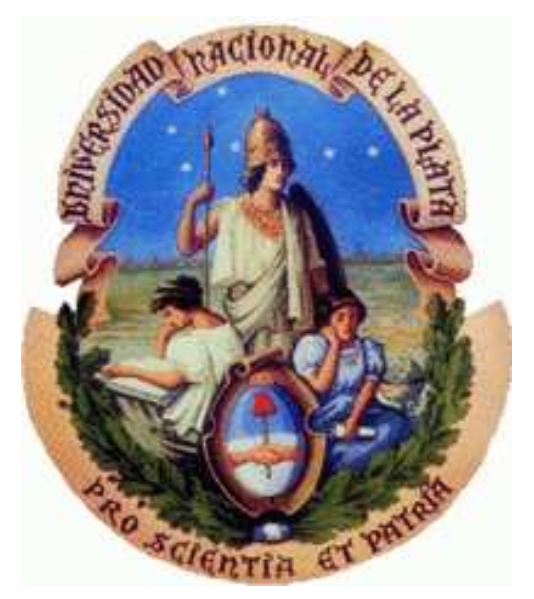

Universidad Nacional de La Plata

\section{EVOLUCIÓN DE GALAXIAS EN CÚMULOS REVELADA A TRAVÉS DE LA RELACIÓN COLOR MAGNITUD}

Tesis para optar por el grado de Doctora en Astronomía

presentada por

Lic. Noelia Jiménez

Directora: Dra. Sofía A. Cora

Febrero de 2012

CON I CET

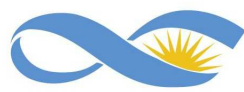


(Reverso de la Tapa) 
Dedico esta tesis a mis padres Virginia y Ricardo, A la memoria de mis abuelos Ñaña y Atico, que se fueron sin permiso. Y al Tata, el abuelo que no llegué a conocer. Además la dedico a mi abuela, Coca, que sigue siguiendo, por suerte. 
[..] Magro y recio a la vez, el astrofísico mostraba empero un aire de laxitud general, como si hubiese desertado viejas y tirantes consignas: la mano que me tendió era blanda y húmeda como el cefalotórax de un molusco; pero en sus ojos grises ardían y se disipaban relámpagos de no fácil diagnóstico. Se dejó caer en un sofá, y la Enviada tomó asiento a su derecha.

- ¿Es usted un científico? - me preguntó él en tono descorazonado.

— El señor Bermúdez es un hombre de letras - le aclaró Bermúdez.

- Menos mal- sentenció Frobenius. Y añadió, en un arranque de ira

— La Ciencia carece de todo "valor explicativo". ¡NO revela un corno! La Enviada le tomó una mano y le dijo:

- ¡Acuérdese, doctor!

- Urania - repuso él-, no estoy agitado. Pero una ciencia arranca de la duda y se dirige a la duda por entre la duda, es un infierno, y uno muy caro. Una verdad que no sea "indudable" no es una verdad. ¿Qué hacemos nosotros? ¿Me dirás que Ciencia? No, señor: nosotros hacemos algo así como el turismo de la duda.

- ¿Quién lo niéga?-le susurró la enviada como si tranquilizase a un niño. El doctor Frobenius dejó caer lentamente su cabeza en el regazo de la mujer:

- Urania - le dijo-, ese metalúrgico tendrá sus cálculos esta noche. ¿Para qué diablos necesitará él todos esos millones de galaxias? ¡A mí me revientan!

Cerró los ojos, y acomodando su cabeza en los muslos de la Enviada pareció adormecerse. Un valet de chaqueta blanca entró como un espectro en el living comedor. Y lentamente comenzó a disponer la mesa en un ángulo del recinto. Sonriendo como lo haría un número, la Enviada nos aconsejó:

- Cenen ustedes.

El doctor Frobenius tomó arriba su vaso de jugo de tomates. Mientras el valet continuaba preparando la mesa, demoré mis ojos en Urania y en el hombre que ya dormía en su regazo. Frobenius tenía razón: con sus pechos de Aritmética, sus muslos pitagóricos y sus manos de abrir compases, la Enviada Número Dos era una imagen viviente de la Astronomía.

Leopoldo Marechal, El Banquete de Severo Arcángelo, 1965. (fragmento) 



\section{Índice general}

$\begin{array}{lr}\text { 1. Introducción } & \mathbf{3}\end{array}$

1.1. Breve reseña histórica . . . . . . . . . . . . . . . . . . . 3

1.2. Contexto y nociones preliminares . . . . . . . . . . . . . . . 4

1.2.1. Secuencia de Hubble-Jeans . . . . . . . . . . . . . . . . . . . 4

1.2.2. Cúmulos y grupos de galaxias . . . . . . . . . . . . . . . . . 4

1.2.3. Relación Color Magnitud . . . . . . . . . . . . . 5

2. Modelo de formación de galaxias $\quad 11$

2.1. Paradigma $\Lambda$ CDM y la formación de estructuras . . . . . . . . . . . . . . 11

2.2. Simulaciones de cúmulos de galaxias . . . . . . . . . . . . . . . . . 12

2.3. Modelo Semianalítico SAG: física de los bariones o "gastrofísica" . . . . . 16

2.3.1. Gas caliente, enfriamiento radiativo, formación estelar y feedback por supernovas . . . . . . . . . . . . . . . . 17

2.3.2. Enriquecimiento químico . . . . . . . . . . . . . . 18

2.3.3. Inestabilidades de disco . . . . . . . . . . . . . . . . . . . . . . . . 19

2.3.4. Fusiones de galaxias . . . . . . . . . . . . . . . . . . . . . 19

2.3.5. Feedback por Núcleos Galácticos Activos . . . . . . . . . . . . . . 21

2.3.6. Modelado de discos galácticos . . . . . . . . . . . . . . . . . 22

2.3.7. Propiedades espectro fotométricas de las galaxias . . . . . . . . . 22

2.3.8. Extinción por polvo interestelar . . . . . . . . . . . . . . 25

2.3.9. Calibración de SAG . . . . . . . . . . . . . 26

3. La Relación Color Magnitud $\quad 29$

3.1. Criterios de selección de galaxias que forman la RCM modelada . . . . . . 29

3.1.1. Criterio de selección basado en la bimodalidad de la distribución de colores ..................... . . 30

3.1.2. Criterio de selección por tipo morfológico . . . . . . . . . . . . 30

3.1.3. Criterio de selección por formación estelar específica . . . . . . . . 33

3.1.4. Discusión sobre los criterios de selección de galaxias . . . . . . . . 34

3.2. Transformaciones entre sistemas fotométricos . . . . . . . . . . . . 36

3.3. La pendiente de la RCM en diferentes bandas fotométricas . . . . . . . . . 39

3.4. Metalicidad de las galaxias de la RCM . . . . . . . . . . . . . . . . 44

4. Procesos físicos involucrados en el desarrollo de la RCM $\quad 47$

4.1. Formación y acreción de la masa estelar . . . . . . . . . . . . . . . . 47

4.2. Definición de nuevas variables . . . . . . . . . . . . . . . . . 50

4.3. Evolución de las fracciones de masa . . . . . . . . . . . . . . . . 51

4.3.1. Influencia de la resolución numérica . . . . . . . . . . . . . . . . . . . . . . . . 56

4.3.2. Influencia del umbral de la fracción de gas . . . . . . . . . . . . . . . . . . . 58

4.4. Evolución de la metalicidad . . . . . . . . . . . . . . . . . . . . 59 
5. Factores que determinan el quiebre de la RCM 63

5.1. Consideraciones respecto de las variables introducidas en SAG . . . . . . 63

5.2. Agregación de masa desde $z=1 \ldots \ldots \ldots$. . . . . . . . . 68

5.3. Comparación con resultados observacionales . . . . . . . . . . . . . 68

5.3.1. Fracciones de masa formada in situ . . . . . . . . . . . . . . . 68

5.3.2. Tasas de fusiones . . . . . . . . . . . . . . . . . . 69

5.4. Contribución de metales desde $z=1 \ldots \ldots \ldots 72 \ldots \ldots 72 \ldots \ldots$

5.5. Discusión de los resultados: Influencia del ambiente . . . . . . . . . . . 73

6. Nuevas implementaciones en SAG: Efectos sobre la RCM $\quad 77$

6.1. Nuevo modelo de extinción por polvo . . . . . . . . . . . . . . . 77

6.1.1. Efectos de la extinción sobre la RCM . . . . . . . . . . . . . . 78

6.1.2. Efectos sobre la Relación Luminosidad Metalicidad . . . . . . . . . 83

6.2. Efectos de la fase TP-AGB en las galaxias . . . . . . . . . . . . . . 83

6.2.1. Nuevos modelos evolutivos de síntesis de poblaciones . . . . . . . . 86

6.2.2. Fase TP-AGB en modelos semianalíticos . . . . . . . . . . . . 86

6.2.3. Efectos de la fase TP-AGB en la RCM . . . . . . . . . . . . . . 87

6.3. Nueva formulación para la formación estelar . . . . . . . . . . . . . . . 88

6.3.1. Influencia de la nueva formulación de SF en la RCM . . . . . . . . 89

$\begin{array}{ll}\text { 7. Conclusiones } & 101\end{array}$

7.1. Agradecimientos . . . . . . . . . . . . . . . . . . . 113 


\section{RESUMEN}

En este Trabajo de Tesis se investigan los procesos involucrados en el desarrollo de la Relación Color Magnitud (RCM) de galaxias en cúmulos. Esta relación comprende galaxias de tipo temprano y corresponde a la secuencia roja (SR) observada en el plano color magnitud. La distribución de galaxias en este plano es bimodal en los colores y parte de las galaxias se distribuyen en una zona difusa llamada "nube azul". La SR está formada mayormente por galaxias pobres en gas con baja formación estelar, prototípicamente galaxias de tipo temprano. Estas galaxias constituyen el objeto de estudio de la presente investigación y la relación color magnitud que las caracteriza será referida como RCM. Esta relación puede entenderse como una correlación entre la masa y la metalicidad de las galaxias, siendo aquéllas más masivas y luminosas, las más metálicas y rojas. Usualmente, se ha utilizado una recta para ajustar la forma funcional de la RCM, que se extiende desde las galaxias elípticas enanas hasta las gigantes. Sin embargo, observaciones recientes muestran que las galaxias más luminosas de la RCM $\left(M_{R} \sim M_{V} \sim M_{T 1} \lesssim-20\right.$, de masa $\left.\mathrm{M} \sim 10^{10} M_{\odot}\right)$, se separan del ajuste lineal seguido por las galaxias menos luminosas mostrando colores prácticamente constantes. Esto resulta en un quiebre del extremo brillante de la RCM hacia colores más azules. En este trabajo se pretende explicar el origen de este quiebre y contribuir a la comprensión de la formación de galaxias en ambientes de cúmulos. Para ello, utilizamos una técnica híbrida que combina simulaciones hidrodinámicas de $N$-cuerpos con un modelo semianalítico de formación de galaxias que tiene en cuenta los procesos físicos que afectan a los bariones. La RCM resultante en diferentes bandas fotométricas se encuentra en buen acuerdo con la tendencia lineal observada. El quiebre en el extremo brillante emerge naturalmente del modelo como resultado de todos los procesos físicos incluídos. Debido a que las galaxias luminosas tiene un dispersión pequeña de edades $\left(1,0 \times 10^{10}\right.$ años $<t<1,2 \times 10^{10}$ años $)$, la metalicidad tiene un rol predominate en la determinación de sus colores. Las abundancias químicas reflejan los procesos físicos involucados en la evolución de las galaxias (brotes estelares, y fusiones "secas" y "húmedas" caracterizadas por bajo y alto contenido de gas, respectivamente). Encontramos que la evolución de las galaxias en el extremo brillante de la RCM está regulada desde $z \sim 2$ principalmente por fusiones menores y mayores de tipo "secas". Por otro lado, las fusiones menores y mayores "húmedas" son relevantes para la determinación de las propiedades de galaxias de menor luminosidad (por debajo del quiebre). Las fusiones galácticas contribuyen con grandes fracciones de masa estelar proveniente de las galaxias satéites $(\sim 10-20$ por ciento), pero las metalicidad de las mismas es $\sim 0,2$ dex más bajas que la metalicidad media de las galaxias centrales con las cuales se fusionan. El efecto de las fusiones secas es, por tanto, incrementar la masa de las galaxias del extremo brillante sin alterar significativamente sus metalicidades. Luego, galaxias muy luminosas muestran colores similares que resultan más azules que los que corresponderían si la formación estelar fuese más elevada o, de manera equivalente, si se extrapolara el comportamiento de las galaxias menos luminosas. Esto explica el quiebre en la RCM, que pone de manifiesto un camino evolutivo diferente para las galaxias más y menos masivas. Hallamos resultados similares al estudiar cúmulos con diferentes masas viriales $\left(10^{14}-10^{15} M_{\odot}\right)$, en acuerdo con la universalidad de la RCM hallada en diferentes muestras observacionales. Para evaluar la robustez de nuestras conclusiones, consideramos otras implementaciones en el modelo semianalítico que incluyen un nuevo modelo de extinción por polvo interestelar, nuevas librerías sintéticas de espectros de poblaciones estelares, y una formulación alternativa que regula la formación estelar, encontrando en todos los casos que nuestros resultados no se alteran significativamente, dado que el quiebre en el extremo brillante de la RCM está presente. 


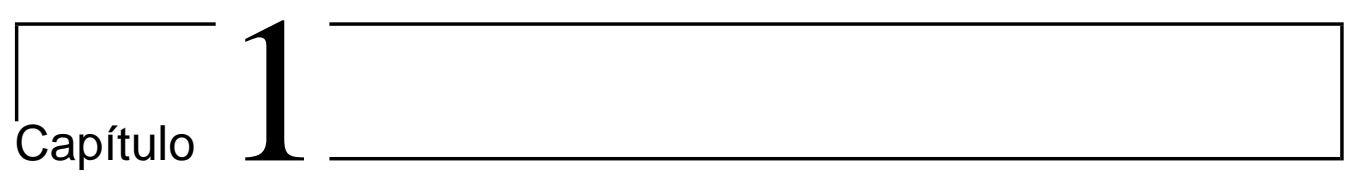

\section{Introducción}

\subsection{Breve reseña histórica ${ }^{1}$}

La existencia de objetos brillantes y difusos en el cielo era un hecho bien conocido ya a fines del siglo XVIII. Estos objetos se diferenciaban de las estrellas porque no podían ser resueltos como fuentes puntuales con los instrumentos de observación de la época. Se los describía como pequeñas nubes. El astrónomo francés Charles Messier, interesado en la búsqueda de cometas, fue el primero en catalogar las "nébulas", para evitar confundirlas con sus objetos de estudio. Este catálogo, "Catalogue des Nébuleuses et des amas d'Étoiles, que l'on découvre parmi les Étoiles fixes sur l'horizon de Paris", fue realizado entre los años 1771 y 1784. Galaxias del Grupo Local, como Andrómeda (M31), llevan hoy en día nombres dados por Messier. Más tarde, en 1864, el astrónomo John Herschel publicó un catálogo que fue actualizado por John Dreyer en 1888. El catálogo "The New General Catalogue of Nebulae and Clusters of Stars (NGC)", junto con dos anexos más "Index Cataloge", presentaba un total de 15000 objetos nebulares clasificados. Las iniciales de los catálogos se utlizan hasta nuestros días para la denominación de objetos celestes; así pues, NGC185 es el nombre de una galaxia elíptica enana en la Constelación de Casiopea.

La naturaleza de las nebulosas fue un tema controversial en la Astronomía del siglo pasado. En 1920, la Academia Nacional de Ciencias de Washington invitó a los dos astrónomos más ilustres de la época, Harlow Shapley y Heber Curtis, a debatir sobre el tema. Shapely sostenía que las nebulosas eran objetos que se ubicaban dentro de la Via Láctea, mientras que Curtis sostenía la idea de que estos objetos eran "universos islas", externos, individuales y similares a la Via Láctea. Esta reunión se conoció luego en la comunidad astronómica como "El Gran Debate" que, irónicamente, tuvo como ganador a Harlow Shapely.

No fue sino hasta 1925, cuando Edwin Hubble utilizó estrellas cefeidas para estimar las distancias a las nebulosas, que se supo que Curtis estaba en lo cierto. Hubble demostró que muchas de las nebulosas eran objetos separados de la Via Láctea, individuales y de tamaños comparables a ella. Esto marcó el inicio de la Astronomía Extragaláctica. Gracias a los instrumentos que se fueron desarrollando con el paso del tiempo, se hizo cada vez más sencillo estudiar a las las "galaxias". Los primeros esfuerzos estuvieron abocados a categorizar y ordenar los diferentes tipos de galaxias. Sir James Jeans fue el primero en presentar un diagrama de clasificación en forma de diapasón, que separaba a las galaxias en "tipo temprano" y "tipo tardío". Este esquema de clasificación realizado en 1928 fue luego popularizado por Hubble en su famosa publicación de 1936. Dicha clasificación sigue vigente en nuestros días.

En 1910, Hertzsprung \& Russell mostraron que contraponer las magnitudes y los

\footnotetext{
${ }^{1}$ Basada en Mo, van den Bosch \& White (2011)
} 
colores de las estrellas reflejaba una distribución no uniforme en ese plano de color magnitud. El estudio de las características particulares del diagrama color magnitud llevó a los astrónomos a especular sobre la idea de que, a través del mismo, se evidenciaban procesos evolutivos en las estrellas. Esta idea resultó ser correcta. Siguiendo con estos lineamientos se desarrolló, más tarde, un conjunto de relaciones de escala de las galaxias, esto es, relaciones entre dos o más de sus propiedades, de manera que las mismas permitiesen obtener información sobre las galaxias. Los estudios pioneros de Visvanathan \& Sandage (e.g. 1977); Sandage \& Visvanathan (e.g. 1978); Bower, Kodama \& Terlevich (e.g. 1998) utilizaron el diagrama color magnitud de las galaxias de grupos, cúmulos y campo, como una herramienta para analizar los procesos evolutivos galácticos.

En esta Tesis, exploramos una región del diagrama color magnitud de galaxias, la Relación Color Magnitud (RCM), con el objetivo de explicar los procesos evolutivos de las galaxias que forman la relación, enfocándonos en las galaxias del extremo brillante de la RCM.

\subsection{Contexto y nociones preliminares}

\subsubsection{Secuencia de Hubble-Jeans}

El esquema de clasificación de galaxias propuesto por Hubble, basado en las ideas de Jeans, separa a las galaxias en dos grandes grupos. Las galaxias de tipo "tardío", a la derecha del diagrama, y las galaxias de tipo "temprano", en la izquierda, conectadas por la clase de galaxias lenticulares o S0.

El grupo de las galaxias de tipo temprano comprende galaxias elípticas que presentan isofotas suaves, casi elípticas, y se subdividen en siete tipos El grupo de las galaxias tardías, por su parte, comprende galaxias de disco, con brazos espirales. Las mismas se subdividen en dos grupos, las que presentan barras o "galaxias barradas" y las que no, llamadas "galaxias normales". A su vez, dentro de cada rama, se subdividen en tres clases de acuerdo a cúan comprimidos se ven los brazos espirales, la fracción de luz del bulbo central y el grado con el que pueden resolverse los brazos en estrellas individuales, regiones HII y senderos de polvo. Al final de la secuencia de Hubble-Jeans se ubican las galaxias irregulares que no presentan simetría alguna, no están dominadas por un bulbo ni por un disco.

En la figura 1.1 puede verse una representación esquemática del la secuencia morfológica con la conocida forma de diapasón. Notablemente, la secuencia se correlaciona con otras propiedades de las galaxias. Se trata, pues, de una clasificación que no es meramente descriptiva, sino que resulta fundamentada en propiedades físicas. Las galaxias elípticas y lenticulares se agrupan juntas bajo el término de galaxias de tipo temprano. Las mismas presentan escasa cantidad de gas frío y polvo, tienen colores fotométricos rojos asociados a poblaciones estelares viejas, y su distribución superficial de brillo es elíptica. Las galaxias elípticas presentan colores y metalicidades bien correlacionados con su luminosidad. Las galaxias espirales e irregulares, se agrupan en lo que se denomina galaxias de tipo tardío. Éstas tiene brazos espirales donde continuamente se forman nuevas estrellas, y poseen grandes cantidades de polvo, regiones HII, y gas molecular; sus colores fotométricos son azules y sus perfiles de brillo superficial se aproximan con una función exponencial, que se asocia a discos delgados.

\subsubsection{Cúmulos y grupos de galaxias}

Los grupos y cúmulos de galaxias son las estructuras virializadas más grandes del Universo y constituyen excelentes laboratorios de estudio de las propiedades y la formación de sus miembros. Los cúmulos pueden clasificarse de acuerdo a su forma, riqueza y tipo de galaxias dominantes, entre otras posibilidades. La división entre grupos y cúmulos no 


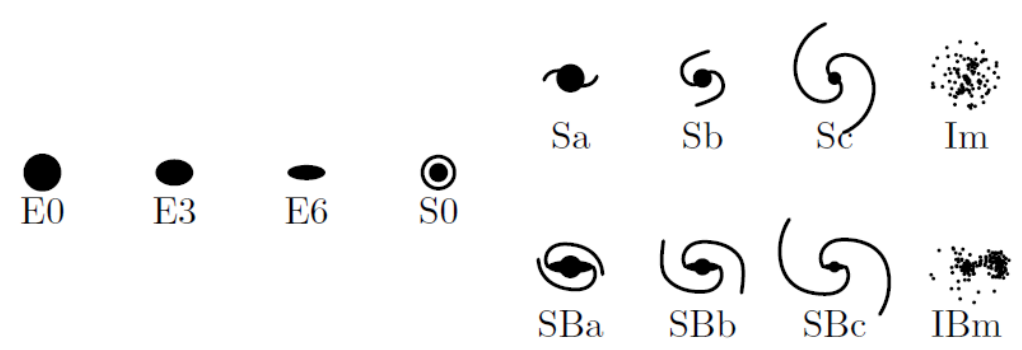

Figura 1.1: Secuencia de Hubble en forma esquemática. Las galaxias de tipo "tardío", a la derecha del diagrama, y las galaxias de tipo "temprano", en la izquierda, conectadas por la clase de galaxias lenticulares o S0.

es clara; un cúmulo de galaxias tiene $50-1000$ miembros brillantes, una masa promedio de $10^{14}-10^{15} M_{\odot}$, y un tamaño de unos pocos $(2-10)$ Mpc de diámetro. Generalmente, los cúmulos son ricos en galaxias de tipo temprano. Los grupos de galaxias, por otra parte, tienen de 3 a 30 galaxias luminosas, su diámetro medio es de 0,1-1 Mpc, y sus masas típicas varían en el rango $10^{12,5}-10^{14} M_{\odot}$. La figura 1.2 muestra la distribución de galaxias, gas caliente y materia oscura de un cúmulo de galaxias que evidencia claros indicios que el mismo se formó a partir de la fusión de cúmulos menores.

\subsubsection{Relación Color Magnitud}

En la actualidad, es un hecho bien establecido que las galaxias forman una distribución bimodal en el plano color magnitud. El diagrama color magnitud (DCM) presenta, por un lado, una estrecha correlación entre los colores y las magnitudes de las galaxias, conocida como "Relación Color Magnitud" (RCM) o Secuencia Roja (SR), mientras que también exhibe una región difusa llamada "nube azul". La RCM está constituida básicamente por galaxias deficientes en gas, con bajos niveles de formación estelar, v.g. galaxias de tipo temprano (ETG, según siglas en inglés, Early Type Galaxies) ${ }^{2}$, mientras que las galaxias de tipo tardío son típicamente objetos de la nube azul. Un ejemplo de la bimodalidad en los colores puede verse en el diagrama color magnitud de la figura 1.3, correspondiente a un relevamiento del Sloan Digital Sky Survey (SDSS) con galaxias en el rango de redshift ${ }^{3}$ $z<0,1$. La figura fue extraída del trabajo de Baldry et al. (2006), quienes estudian la distribución de galaxias en el DCM, sin efectuar cortes por morfología, ajustándolas con distribuciones gaussianas. Encuentran que la RCM no está bien ajustada por una recta. Los autores proponen un ajuste en función de una recta más una función hiperbólica. El punto de transición de la RCM está alrededor de la magnitud $M_{r}=-19,8$, que coincide con una masa típica de $2 \times 10^{10} M_{\odot}$. La bimodalidad en los colores del DCM persiste hasta por lo menos $z \sim 1$ (Strateva et al., 2001; Blanton et al., 2003; Baldry et al., 2004, 2006).

Existe una gran controversia respecto a cuáles son los mecanismos responsables de la evolución de las galaxias hacia la RCM. Los procesos físicos usualmente involucrados pueden separarse en internos y externos. Los primeros incluyen los procesos de formación estelar pasiva e inestabilidades de disco, mientras que los segundos involucran interacciones entre galaxias y fusiones galácticas (Lin et al., 2010; Robaina et al., 2010), efectos ambientales como interacciones gravitatorias rápidas ("acoso galáctico" o "galaxy harassment" como es conocido en la literatura, Moore et al. 1999), remoción del reservorio de

\footnotetext{
${ }^{2}$ Notar que varios términos serán referidos según sus siglas en inglés por familiaridad con la literatura científica, a saber, ETGs, SF, SFR, AGN, IMF, LF

${ }^{3}$ Se mantendrá la nomenclatura en inglés de varios términos, como redshift, feedback, ram pressure stripping, track evolutivo; los mismos se mostrarán en itálica sólo la primera vez que aparecen.
} 


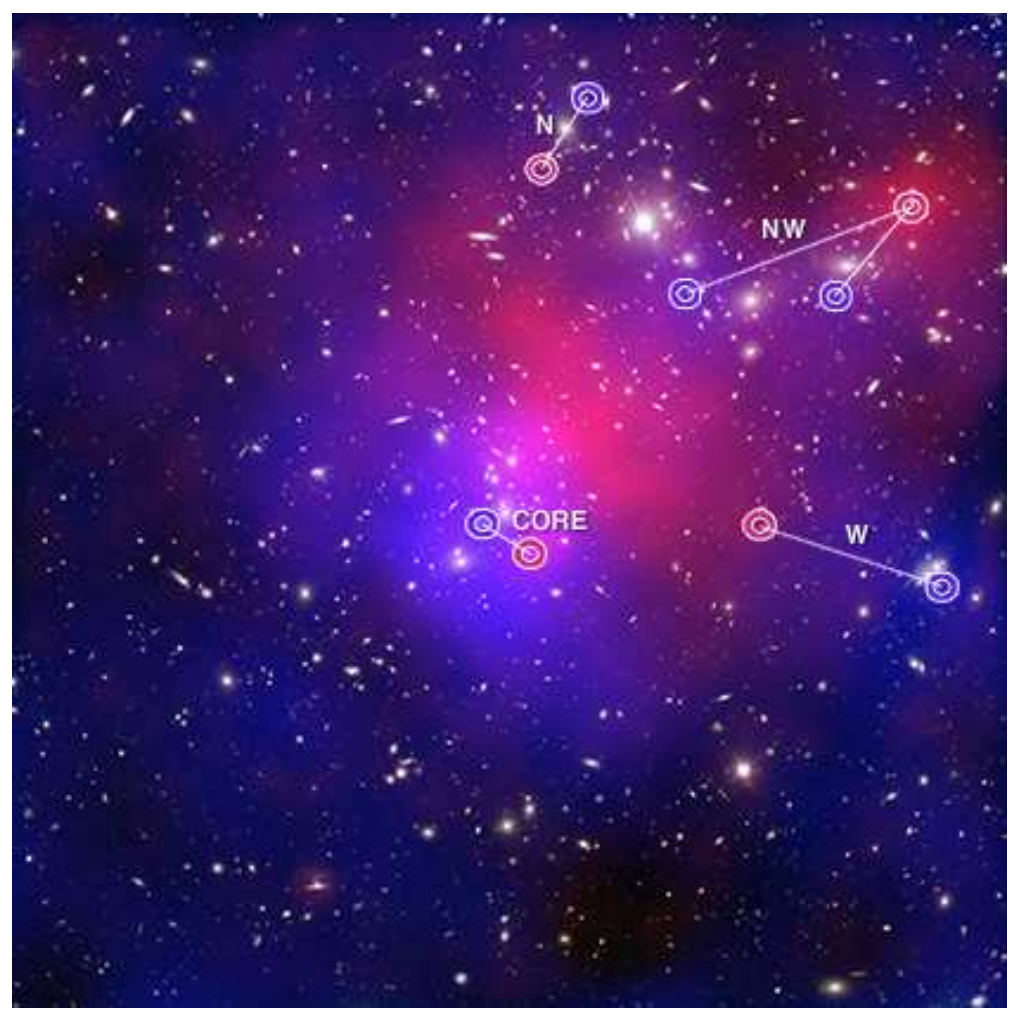

Figura 1.2: Imagen compuesta del cúmulo de galaxias Abell 2744, denominado cúmulo de Pandora, que combina datos del Hubble Space Telescope, del Very Large Telescope perteneciente al European Southern Observatory, del telescopio Japonés Subaru, y del Observatorio de rayos-X Chandra. Los dos primeros aportan las imágenes de galaxias, las cuales constituyen $<5$ por ciento de la masa total del cúmulo. El gas caliente, emisor de rayos-X y detectado por Chandra, aparece en color rojo; el mismo representa $\sim 20$ por ciento de la masa del cúmulo. La distribución de la materia oscura, inferida a partir del fenómeno de lente gravitacional, es coloreada en azul. Este cúmulo gigante parece ser el resultado de la fusión de al menos cuatro cúmulos más pequeños. Los datos sugieren que la compleja colisión ha separado parte del gas caliente, que interactúa durante la colisión, de la materia oscura, de modo que estas dos componentes aparecen apartadas entre sí y de las galaxias visibles. 


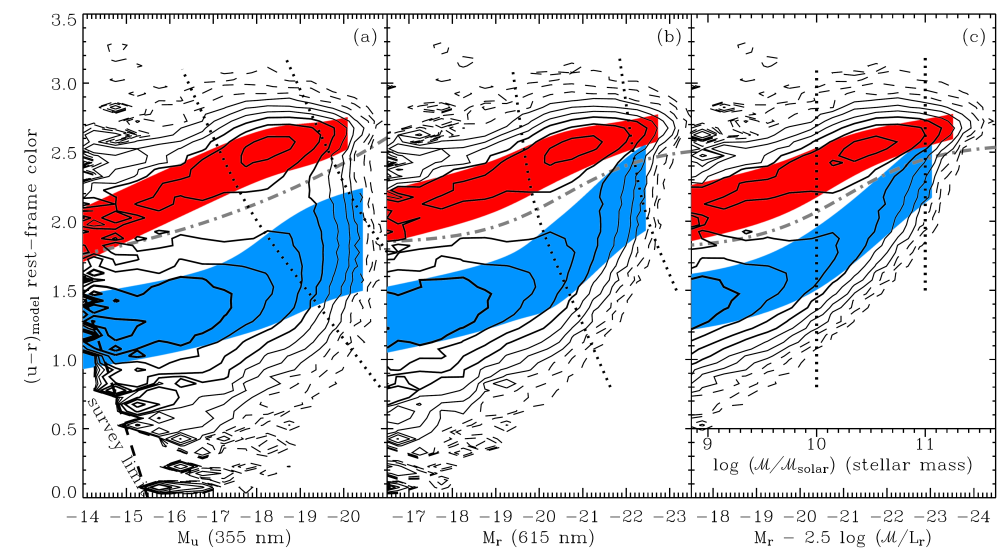

Figura 1.3: Diagramas color magnitud tomados del trabajo de Baldry et al. (2006), basados en el relevamiento de SDSS para $z<0,1$. Los contornos en líneas punteadas y llenas representan densidades de galaxias, espaciadas logarítmicamente con 4 contornos por cada factor 10. Las líneas punteadas, en sentido vertical, representan galaxias de la misma masa estelar correspondiente a $10^{10-11} M_{\odot}$. Las regiones coloreadas representan valores medios para la secuencia roja y azul, más una dispersión de $\pm 1 \sigma$.

gas caliente (denominado "estrangulación" Larson et al. 1980; Balogh et al. 2000; Kawata \& Mulchaey 2008), y la extracción por presión hidrodinámica ("ram pressure stripping", RPS) del gas galáctico (Roediger, 2009; Tecce et al., 2010).

La relación color magnitud puede ser entendida como una relación entre la masa y la metalicidad. Las galaxias más luminosas (y por lo tanto, más masivas) poseen pozos de potencial profundos, los cuales son capaces de retener los metales eyectados por los eventos de supernovas y vientos estelares. La RCM parece ser universal, dado que es seguida tanto por las galaxias de campo como por las de grupos y cúmulos de galaxias (e.g. Visvanathan \& Sandage, 1977; Sandage \& Visvanathan, 1978; Reda et al., 2004; López-Cruz et al., 2004; Reda et al., 2005; de Rijcke et al., 2009). Sin embargo, una mayor proporción de galaxias rojas se encuentra en ambientes de alta densidad.

El estudio de la evolución de la pendiente y la dispersión de la RCM provee restricciones a la historia de formación estelar de las ETGs. Bower, Kodama \& Terlevich (1998) encuentran que se puede reproducir la dispersión observada de la RCM en cúmulos de galaxias locales si se supone que la mayoría de las estrellas en estas galaxias se formaron en épocas tempranas $(z>1)$, con un crecimiento posterior y moderado de la masa causado por fusiones galácticas y una residual formación estelar (SF, según siglas en inglés, Star Formation). Este escenario es consistente con modelos jerárquicos de formación de estructuras.

En este contexto, usando un modelo semianalítico de formación de galaxias, Menci et al. (2008) obtienen una ceñida RCM para las galaxias de cúmulos, definida en tiempos tan tempranos como $z \sim 1,2$. En valores similares de redshift, Kaviraj et al. (2005) encuentran que la pendiente de la RCM de los cúmulos de galaxias cambia apreciablemente respecto al valor mostrado en el presente, aunque la evolución de la pendiente en el rango de redshift $0<z<0,8$ es prácticamente despreciable si se tienen en cuenta los errores de medición.

Observaciones detalladas de la RCM de cúmulos de galaxias (ver Mei et al. 2009, y referencias allí citadas) muestran que no hay una evolución significativa del punto de cero, de la pendiente o la dispersión de la RCM, luego de $z \sim 1,3$. En contraste con estos resultados, Stott et al. (2009) encuentran evolución en los valores de la pendiente de la RCM entre $z \sim 0,5$ y la época actual, en base a observaciones de cúmulos de galaxias en 
el rango del óptico e infrarrojo cercano. Los autores atribuyen esta evolución a la caída de galaxias en el núcleo del cúmulo, que se transforman en galaxias de la secuencia roja.

Las simulaciones hidrodinámicas también han sido usadas para analizar las propiedades de la RCM a $z=0$. Saro et al. (2006) evaluan el impacto de la función inicial de masa estelar (IMF, según siglas en inglés, Initial Mass Function), encontrando que la función de Salpeter permite reproducir tanto la pendiente como la normalización de la RCM para galaxias que habitan en cúmulos. Del análisis de simulaciones de grupos y cúmulos de galaxias, Romeo et al. (2008) concluyen que la forma de la secuencia roja está principalmente determinada por la SF específica en todos los ambientes. Estos autores encuentran que las galaxias evolucionan hacia una secuencia "muerta", justo después de que agotan el reservorio de gas frío del cual forman estrellas. Las galaxias más débiles de los cúmulos mantienen una tasa de SF significativa hasta épocas recientes, y por ello se distribuyen de manera menos acotada en torno a la RCM.

Generalmente, se ha utilizado una relación lineal para ajustar la correlación entre luminosidad y color de las galaxias de la RCM, que se extiende desde las galaxias elípticas enanas a las elípticas gigantes. Sin embargo, la dispersión de estas relaciones aumenta a menores luminosidades, tal como resulta evidente de las observaciones de varios cúmulos galácticos (Coma, Secker et al. 1997; Perseus, Conselice et al. 2003; Fornax, Hilker et al. 2003, Karick et al. 2003, Mieske et al. 2007; Hydra, Misgeld et al. 2008; Virgo, Lisker et al. 2008; Antlia, Smith Castelli et al. 2008).

Algunas de estas relaciones parecen ser consistentes con un cambio en la pendiente al pasar del extremo débil al extremo brillante de la RCM. Este tipo de ajuste ya fue sugerido por de Vaucouleurs (1961) para el cúmulo de Virgo y fue confirmado posteriormente por Ferrarese et al. (2006) Más recientemente, basados en los datos del SDSS, Janz \& Lisker (2009) encontraron, también para el cúmulo de Virgo, una relación no lineal que puede ser descripta como una forma de "S" a lo largo de todo el rango de magnitudes, con las galaxias más brillantes $\left.\left(-21 \lesssim M_{\mathrm{B}} \lesssim-19\right)\right)$ mostrando un color casi constante. Para el cúmulo de Hydra, Misgeld et al. (2008) ajustan una relación lineal a su RCM, sin embargo, es evidente un cambio de pendiente en el extremo brillante de la relación.

A partir de estudios de grandes muestras de galaxias de tipo temprano en el SDSS (e.g. Baldry et al., 2004, 2006; Skelton et al., 2009), surge evidencia adicional de una inclinación hacia colores más azules en el extremo brillante de la RCM. Los resultados de Skelton et al. (2009) corresponden a una RCM del universo local, obtenida promediando sobre todos los ambientes. Utilizando como ajuste de la RCM la combinación de una recta y una función de tangente hiperbólica (que da la forma de una "S"), Baldry et al. (2006) examinan la dependencia del mismo con las posiciones medias de la RCM y de la nube azul, encontrando que, a diferencia de lo que sucede con la fracción de galaxias rojas, el ajuste a la RCM no varía fuertemente con el ambiente.

En cuanto a las propiedades de las galaxias más brillantes que pueblan la RCM, existen observaciones recientes que apoyan la existencia de una fuerte evolución con el tiempo cósmico de las propiedades estructurales de las galaxias esferoidales más masivas (i.e., con masas estelares de $\left.M_{\star} \gtrsim 10^{11} M_{\odot}\right)$. Las mediciones de los tamaños de las galaxias elípticas a altos redshifts $(z \gtrsim 1,5)$ revelan que estos objetos son mucho más pequeños (por un factor de $\sim 2$ a $\sim 4$ ) que sus contrapartes locales de masas similares (Daddi et al. 2005). Este resultado ha sido confirmado subsecuentemente por varios estudios, aunque las galaxias de tipo temprano más masivas $\left(M_{\star} \gtrsim 2,5 \times 10^{11} M_{\odot}\right)$ parecen haber alcanzado su estructura dinámica e interna más rápido y temprano que aquellas ETGs de menor masa, a igual redshift (Mancini et al., 2010). Por otro lado, se encuentra una evolución moderada en la dispersión de velocidades entre las galaxias más masivas (Cenarro \& Trujillo, 2009). En promedio, la dispersión de velocidades y la densidad de masa superficial de galaxias masivas a altos redshifts son similares a aquéllas encontradas en las ETGs locales más densas (Cappellari et al., 2009). Estas evidencias observacionales sugieren que los escenarios de crecimiento "de adentro hacia afuera" son plausibles. En estos escenarios, las galaxias compactas a altos redshifts conforman el centro de las galaxias cercanas elípticas normales (van Dokkum et al., 2010). 
A partir de muestras de pares de galaxias obtenidos de diferentes relevamientos, se ha encontrado que las ETGs masivas locales sufren en promedio $\sim 0,5$ fusiones galácticas desde $z \sim 0,6-0,7$ en adelante (Bell et al., 2006b; Robaina et al., 2010). Estas fusiones galácticas ocurren generalmente entre galaxias que ya están en la secuencia roja, por lo que involucran galaxias de tipo temprano que contienen poco gas frío y mucho polvo (Whitaker \& van Dokkum, 2008; McIntosh et al., 2008). Estas fusiones galácticas mayores no disipativas (deficientes en gas, y por lo tanto llamadas fusiones "secas") son un canal importante para la formación y evolución de las galaxias más brillantes de los cúmulos (BCGs, según sigla en inglés, Brightest Cluster Galaxies) (Liu et al., 2009). Sin embargo, la tasa de fusiones mayores secas es demasiado baja para explicar completamente la formación de galaxias esferoidales (y la secuencia roja) desde $z \sim 1$ (Bundy et al., 2009). Las fusiones secas menores favorecerían el crecimiento de las BCGs tal como se puede inferir a partir de la evolución de la relación entre la dispersión de velocidades y la masa estelar (Bernardi, 2009). La hipotétsis de las fusiones menores también parece ser una explicación plausible para las ETGs, en redshifts intermedios $(0,1<z<0,8)$, tal como lo muestran Nierenberg et al. (2011). Ellos encuentran que las ETGs están rodeadas, típicamente, por varias galaxias satélites. Las fusiones menores son posibles responsables de la evolución del tamaño de las galaxias de tipo temprano masivas (Bezanson et al., 2009). Además, Kaviraj et al. (2008) demuestran que el bajo nivel de formación estelar en la población de ETGs a redshifts intermedios es muy posiblemente debida a la acción de las fusiones galácticas menores.

La influencia de las fusiones galácticas en la evolución de las ETGs, inferida de datos observacionales, está en acuerdo con las predicciones de los modelos de formación de galaxias. Utilizando una técnica semianalítica, Khochfar \& Burkert (2003) encuentran que la última fusión mayor de las galaxias elípticas brillantes del Universo local $\left(M_{B} \lesssim-21\right)$ ocurren preferentemente entre galaxias dominadas por bulbos. Las fusiones mayores son responsables de duplicar la masa estelar de galaxias en el extremo brillante de la RCM desde $z \sim 1$ (Eliche-Moral et al., 2010). Por otro lado, Bournaud, Jog \& Combes (2007) estudian el efecto de múltiples fusiones menores en la evolución de las ETGs mediante un código numérico de $N$-cuerpos, encontrando que las fusiones menores repetidas pueden formar galaxias elípticas sin la necesidad de involucrar fusiones mayores, los cuales son menos frecuentes que las fusiones menores a redshifts moderados. Los remanentes de estas fusiones múltiples tienen propiedades que dependen más de la masa total acretada durante las fusiones que del cociente de masas de cada fusión en particular. Este resultado también es respaldado por simulaciones hidrodinámicas (Naab et al., 2007, 2009), de las cuales se desprende que el crecimiento de las galaxias masivas $\left(M_{\star} \gtrsim 10^{11} M_{\odot}\right)$ mediante una serie de muchas fusiones menores se vuelve más importante que el crecimiento por fusiones mayores. Las fusiones menores, pues, resultan ser la principal fuente en la evolución tardía de los tamaños y densidades de las ETGs. Otro posible mecanismo físico responsable de la evolución del tamaño de las ETGs es la expulsión de una importante fracción de bariones, todavía en forma gaseosa, mediante la actividad de quásars (Fan et al., 2010).

A partir de un modelado semianalítico, Khochfar \& Silk (2006) encuentran que las galaxias masivas a altos redshifts se forman en fusiones ricas en gas, mientras que galaxias de igual masa a bajos redshifts son el resultado de fusiones pobres en gas. Estas consideraciones les permiten reproducir la evolución observada del tamaño de los objetos esferoidales masivos con el redshift. La disipación asociada con las grandes fracciones de gas es el factor más importante en la determinación de los tamaños de las galaxias esferoidales, tal como ha sido encontrado por Hopkins et al. (2009) con la utilización de simulaciones hidrodinámicas. Complementariamente, utilizando métodos semianalíticos, De Lucia \& Blaizot (2007) encuentran que las fusiones menores secas tardías $(z<0,5)$ juegan un rol importante en el ensamblado de masas de las BCGs, las cuales adquieren la mitad de su masa mediante la agregación de galaxias más pequeñas.

Es claro que diferentes tipos de fusiones tienen un enorme impacto en la evolución de las ETGs, afectando sus morfologías y propiedades dinámicas, así como su historia de 
formación estelar. Muchos trabajos se han dedicado a estudiar estos aspectos, pero sólo unos pocos han analizado el rol de las fusiones en el desarrollo de la RCM (e.g. Skelton et al., 2009; Bernardi et al., 2010). Por medio de un modelo muy simple, combinado con los árboles de fusiones de galaxias extraídos de un modelo semianalítico, Skelton et al. (2009) encuentran una explicación para el apartamiento hacia colores azules del extremo brillante de la RCM. Los autores sostienen que el mismo se debe a que existe una fracción mayor de fusiones menores secas sufridas por las galaxias brillantes en relación a las débiles. El color de las galaxias no debería cambiar debido a que en las fusiones secas no se produce formación estelar. Por ello, las galaxias se mueven a lo largo de la RCM a medida que la masa se incrementa, mientras los colores permanecen fijos (Bernardi et al., 2007). En esta Tesis, investigamos este aspecto en detalle para encontrar una demostración o una falsación a dicha hipótesis, según sea el caso.

El apartamiento del extremo brillante de la RCM con respecto a la tendencia marcada por el ajuste lineal a toda la relación motiva este Trabajo de Tesis. La RCM constituye una de las mayores herramientas para evaluar los modelos de formación de galaxias ya que la evolución de las galaxias se evidencia a través de la misma. Nuestro objetivo es contribuir al entendimiento de los procesos físicos involucrados en el desarrollo de la RCM de cúmulos de galaxias, y del comportamiento especial mostrado por su extremo brillante. Nuestro método de trabajo incluye la aplicación de un modelo semianalítico de formación y evolución de galaxias SAG (Cora, 2006; Lagos, Cora \& Padilla, 2008; Tecce et al., 2010, acronismo para Semi-Analytic Galaxies;) a un conjunto de simulaciones cosmológicas de cúmulos de galaxias (Dolag et al., 2005).

Esta Tesis se diagrama de la siguiente forma. En el capítulo 2 se describe el marco cosmológico en el que se desarrolla esta investigación, y se describen las simulaciones de cúmulos de galaxias utilizadas, y los principales procesos físicos incluidos en el modelo semianalítico empleado. En el capítulo 3 se presenta la RCM obtenida según diferentes criterios de selección de galaxias de tipo temprano, y se discute la comparación de las pendientes de ajuste a la RCM en varias bandas fotométricas con valores reportados por estudios observacionales. A fin de identificar qué mecanismos son responsables de las diferentes características mostradas por la RCM y, en particular, del quiebre en el extremo brillante, en el capítulo 4 presentamos el estudio de la evolución de las masas y metalicidades de la componente estelar de cada galaxia como resultado de diferentes tipo de eventos que conducen a la formación y acreción de estrellas. A partir de los resultados obtenidos en este capítulo, en el capítulo 5 se evaluan las caraterísticas de la masa estelar acumulada en las galaxias desde $z=1$ al presente, a fin de poder explicar claramente la razón física de la presencia del quiebre de la RCM. El capítulo 6 está dedicado al estudio del impacto sobre las propiedades de la RCM de nuevas implementaciones incluidas en el modelo semianalítico SAG, referidas a la corrección por polvo de las magnitudes resultantes, a la modificación de esas magnitudes por incluir la emisión de estrellas en la fase TP-AGB, y a la consideración de una formulación alternativa para describir el proceso de formación estelar. Finalmente, presentamos nuestras conclusiones en el capítulo 7 .

Cabe destacar que el análisis y los resultados descriptos en los capítulos 3,4 , y 5 se desprenden del trabajo de Jiménez et al. (2011). 


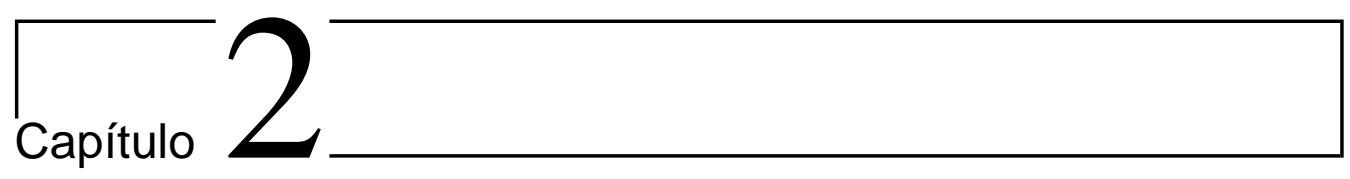

\section{Modelo de formación de galaxias}

En este trabajo investigamos las razones físicas que determinan las características de la RCM de cúmulos de galaxias, enfocando nuestra atención en el comportamiento del extremo brillante de esta relación. El estudio se aborda desde un punto de vista teórico, utilizando como herramienta una técnica numérica híbrida que combina simulaciones cosmológicas no radiativas $N$-Body/SPH (Smoothed Particle Hydrodynamics) de cúmulos de galaxias con el modelo semianalítico de formación de galaxias SAG . En este capítulo se detallan las simulaciones de cúmulos de galaxias utilizadas, y se describen los principales procesos físicos incluidos en el modelo semianalítico empleado que afectan a la componente bariónica, y el marco cosmológico en el que se aplica.

\subsection{Paradigma $\Lambda$ CDM y la formación de estructuras ${ }^{1}$}

El modelo estándar de formación y evolución del Universo es el resultado de una exitosa combinación de modelos pertenecientes a la Cosmología y a la Astronomía. El paradigma vigente propone un Universo de materia oscura fría con constante cosmológica, llamado según sus siglas en inglés $\Lambda$ Cold Dark Matter $(\Lambda \mathrm{CDM})$. Este escenario ha integrado armoniosamente teorías cosmológicas (Teoría del Big Bang y de Inflación), modelos físicos de partículas (Modelo Estándar y sus extensiones), modelos astrofíscos (crecimiento de estructuras cósmicas por efectos gravitatorios, crecimiento jerárquico, "gastrofísica"), y fenomenología (anisotropías del Fondo Cósmico de Radiación, materia oscura no bariónica, energía oscura de naturaleza repulsiva, geometría plana y propiedades galácticas).

Los picos acústicos detectados en el espectro de potencias del Fondo Cósmico de Microondas (CMB, según siglas en inglés, Cosmic Microwave Background) mostraron un acuerdo perfecto con las predicciones del modelo $\Lambda$ CDM. En el año 2003, los datos del satélite WMAP no sólo confirmaron este resultado sino que hicieron más precisa la determinación de los parámetros cosmológicos. Los valores obtenidos están en un acuerdo notablemente bueno con medidas independientes; la densidad de bariones acuerda con la predicha según los modelos de nucleosíntesis primordial, la constante de Hubble $H_{0}$ se corresponde con la hallada por medidas directas, la densidad de energía oscura acuerda con la obtenida de mediciones de supernovas tipo Ia, y la estructura a gran escala del Universo observada está de acuerdo con los resultados de grandes muestreos de galaxias y lentes gravitacionales débiles (ver Spergel et al. 2003).

En este escenario, las estructuras se forman a partir de perturbaciones iniciales a un medio homogéneo e isotrópico en expansión. Las mismas se relacionan con las fluctuaciones cuánticas del campo escalar, creadas en el período inflacionario del Universo.

\footnotetext{
${ }^{1}$ Basado parcialmente en Avila Rees (2007) y Mo, van den Bosch \& White (2011)
} 
Las pequeñas perturbaciones crecen amplificadas por la gravedad, de acuerdo a una ley de potencias (en un universo de Einstein-de Sitter). Cuando la densidad de materia del Universo iguala a la densidad de radiación $\left(z_{e q}\right)$, las pertubaciones de materia oscura comienzan a crecer. En el momento del desacople de la materia y la radiación, la materia bariónica aumenta más todavía el crecimiento de las sobredensidades. Éstas, eventualmente, alcanzan un valor crítico y se desacoplan de la expansión del Universo, formando estructuras que colapsan y se virializan. Las estructuras mayores se forman mediante el ensamblaje de estructuras menores, en lo que se denomina un escenario de crecimiento jerárquico o "bottom up".

Press \& Schechter (1974) desarrollan un formalismo para calcular en este régimen la función inicial de masa en función del tiempo (por unidad de volumen comóvil) de los objetos que se desprenden de la expansión general del Universo y colapsan, llamados "halos de materia oscura". Los autores consideran una distribución de densidad de fluctuaciones gaussianas. El crecimiento y colapso de estas estructuras cósmicas es un proceso altamente no lineal, sin simetrías y de una gran escala dinámica. Por ello, simulaciones de $N$-cuerpos son necesarias para su estudio. De los resultados numéricos es posible extraer las historias de formación de los halos de materia oscura.

Un importante resultado de las simulaciones de $N$-cuerpos es la demostración de la existencia de "subhalos", que son halos de materia oscura existentes dentro del radio virial de halos más grandes. Los subhalos de materia oscura sobreviven como estructuras ligadas al colpaso gravitacional de estructuras de niveles de jerarquía más altos. Los subhalos sufren de una pérdida importante de masa debida a las fuerzas de marea. Los torques inducidos por estas fuerzas imprimen momento angular tanto a la materia oscura como a la bariónica. Esta última, inicialmente en forma gaseosa, se enfría condensándose en las regiones centrales, en principio conservando su momento angular, y favoreciendo la formación de las galaxias.

Desde el principio de los años 90 se han investigado las propiedades de los halos de materia oscura a través de dos técnicas numéricas complementarias. Por un lado, existen modelos semianalíticos, originalmente desarrollados por White \& Frenk (1991) y más tarde refinados por numerosos estudios (Kauffmann, White \& Guiderdoni, 1993; Cole et al., 1994; Somerville \& Primack, 1999; Kauffmann et al., 1999; De Lucia et al., 2004; Croton et al., 2006; Cora, 2006; Somerville et al., 2008; Lagos et al., 2008; Guo \& White, 2009). Estos modelos utilizan información sobre la estructura y el ensamblaje de los halos de materia oscura fría para modelar los pozos de potencial dentro de los cuales se forman y evolucionan las galaxias, tratando los procesos físicos (enfriamiento del gas, formación estelar, feedback de supernovas y núcleos galácticos activos), en forma semianalítica. Por otro lado, en el marco dado por $\Lambda$ CDM, Navarro \& White (1994) realizaron por primera vez simulaciones hidrodinámicas a partir de condiciones iniciales cosmológicas. Desde entonces, la resolución y el poder de cómputo de los cúmulos de computadoras ha aumentando enormemente, permitiendo avanzar en el conocimiento de la formación de las galaxias.

\subsection{Simulaciones de cúmulos de galaxias}

En este trabajo utilizamos simulaciones cosmológicas no radiativas $N$-Body/SPH de cúmulos de galaxias, provistas por el Dr. Klaus Dolag (Dolag et al., 2005). Las mismas proporcionan las historias de formación de los halos y subhalos de materia oscura, permitiendo armar los árboles de fusiones (merger trees) a lo largo de la evolución del Universo. Esta información es usada por el código semianalítico para generar la población de galaxias.

Los cúmulos utilizados se seleccionan inicialmente de una simulación que involucra solamente a la materia oscura, con una caja de tamaño de $480 h^{-1} \mathrm{Mpc}$ (Yoshida et al., 2001) en un modelo cosmológico caracterizado por los parámetros $\Omega_{\mathrm{m}}=0,3, \Omega_{\Lambda}=0,7$, 


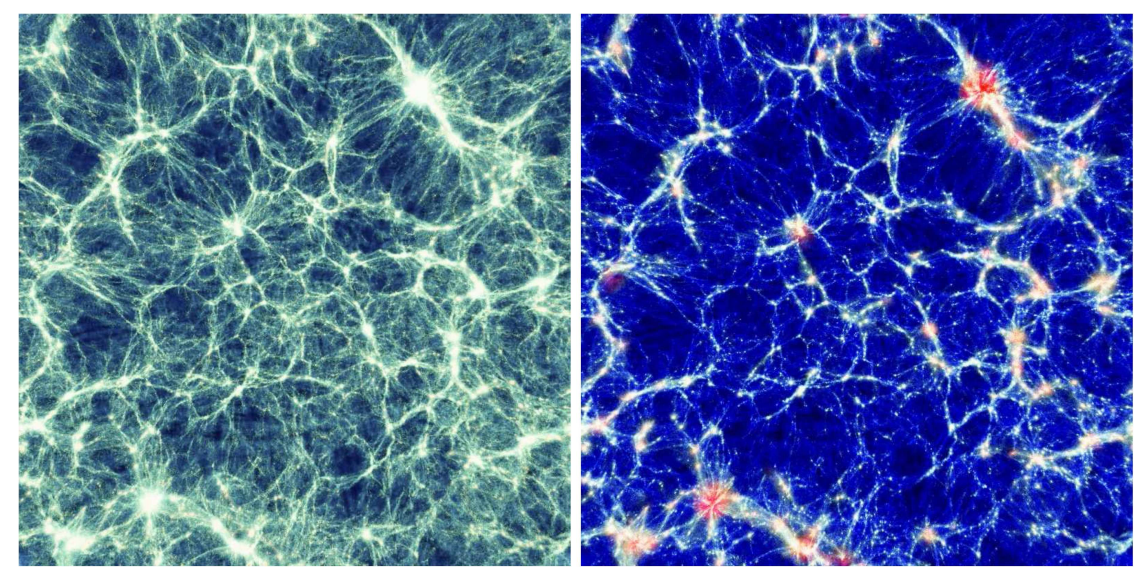

Figura 2.1: Mapa de la densidad de gas en toda la caja de la simulación cosmológica a $z=0$. Para atravesar la caja de lado a lado, un rayo de luz tardaría cerca de $10^{9}$ años. El corte tiene una profundidad de $12 h^{-1} \mathrm{Mpc}$. La estructura más grande que se forma en esta simulación es un cúmulo de galaxias, que puede verse en el margen superior derecho de ambos paneles (Borgani et al., 2004; Dolag et al., 2005). Panel izquierdo: Se muestran regiones de baja y alta densidad de partículas en colores oscuros y claros, respectivamente. Panel derecho: Se aprecia la temperatura del gas dentro de las estructuras cosmológicas, donde el color rojo representa el gas más caliente.
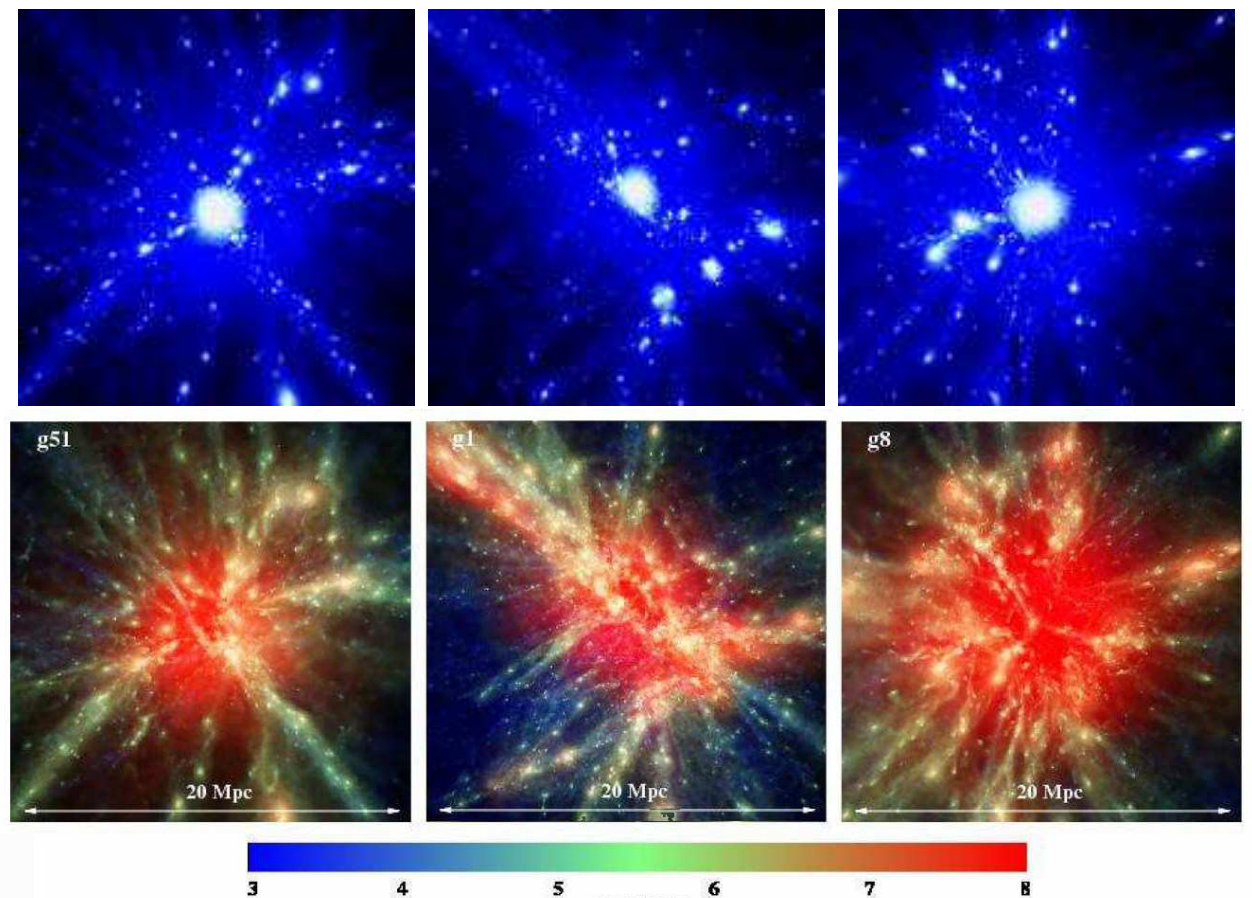

4

5

$\log (\mathbf{T})[\mathbf{K}]$

Figura 2.2: Simulaciones hidrodinámicas no radiativas de la región central de los cúmulos masivos, set C15, realizadas con el código SPLOTCH. En los paneles superiores se muestra la distribución de la materia oscura y gas de los cúmulos g51, g1 y g8. En los paneles inferiores se muestra la temperatura del gas para los mismos cúmulos. (Créditos: http://www.mpa-garching.mpg.de/galform/data). 

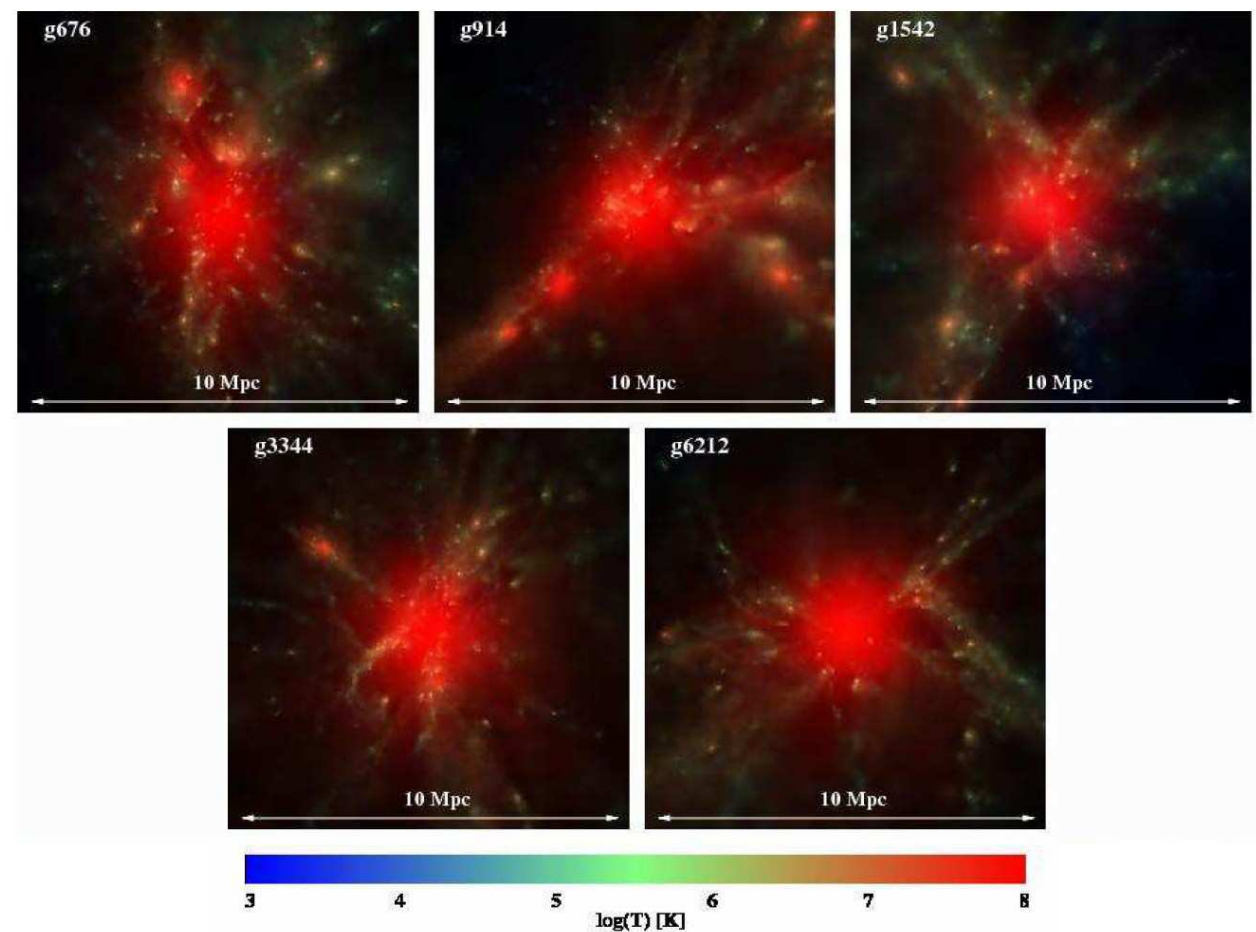

Figura 2.3: Simulaciones hidrodinámicas no radiativas de la región central de los cúmulos menos masivos, set C14. En los paneles superiores se vizualiza la temperatura del gas para los cúmulos g676, g914, g1542, mientras que en los paneles inferiores se presentan las distribuciones de temperatura correspondientes a los cúmulos g3344 y g6212. 


\begin{tabular}{lrr} 
Cúmulo & $R_{\text {vir }}(\mathrm{Mpc})$ & $M_{\text {vir }}\left(10^{14} M_{\odot}\right)$ \\
\hline \hline $\mathrm{C} 14_{1}$ (g1542) & 1,04 & 1,30 \\
$\mathrm{C} 14_{2}$ (g3344) & 1,07 & 1,39 \\
$\mathrm{C} 14_{3}$ (g6212) & 1,06 & 1,31 \\
$\mathrm{C} 14_{4}$ (g676) & 1,06 & 1,33 \\
$\mathrm{C} 14_{5}$ (g914) & 1,09 & 1,43 \\
$\mathrm{C} 15_{1}$ (g1) & 2,50 & 17,80 \\
$\mathrm{C} 15_{2}$ (g8) & 2,89 & 27,56 \\
$\mathrm{C} 15_{3}$ (g51) & 2,37 & 15,30
\end{tabular}

Cuadro 2.1: Propiedades de los cúmulos de galaxias simulados por Dolag et al. (2005). Se muestran las masas y radios viriales a $z=0$ de los cúmulos centrales de cada simulación; las simulaciones que contienen a los cúmulos $g 1$ y $g 8$ tienen, a su vez, cúmulos satélites de masas del orden de $10^{13-14} M_{\odot}$.

$\mathrm{H}_{\mathrm{o}}=70 \mathrm{~km} \mathrm{~s}^{-1} \mathrm{Mpc}^{-1}, \Omega_{\mathrm{b}}=0,039$ para el parámetro de densidad bariónica, y $\sigma_{8}=0,9$ para la normalización del espectro de potencias. Los valores de $\Omega_{m}$ y $\sigma_{8}$ son levemente mayores que los obtenidos del análisis de los datos del quinto año del satélite WMAP (Dunkley et al., 2009), pero aún así son consistentes con las cotas observacionales actuales.

Consideramos dos clases de cúmulos extraídos de la simulación original. Por un lado, el set de cúmulos C14 que contienen cúmulos de masas viriales en el rango $\simeq(1,1-1,2) \times$ $10^{14} h^{-1} \mathrm{M}_{\odot}$ y se compone de cinco miembros a los que denominamos g1542, g3344, g6212, g676 y g914. Por otro lado, consideramos el set de cúmulos C15 de masas viriales en el rango $\simeq(1,3-2,3) \times 10^{15} h^{-1} \mathrm{M}_{\odot}$, que se compone de tres miembros denominados g1, g8, y g51. En la tabla 2.1 se muestran algunas de las propiedades de los estos cúmulos (ver Dolag et al., 2005, para más detalles).

Las regiones lagrangianas que rodean a los cúmulos seleccionados han sido resimuladas con una mayor resolución de masa mediante la técnica "Zoomed Initial Condition" (Tormen et al., 1997) y el código Tree-SPH GADGET-2 (Springel et al., 2005). La resolución de masa es la misma para todas las simulaciones, con masas de materia oscura y partículas de gas de $m_{\mathrm{dm}}=1,13 \times 10^{9} h^{-1} \mathrm{M}_{\odot}$ y $m_{\text {gas }}=1,69 \times 10^{8} h^{-1} \mathrm{M}_{\odot}$, respectivamente. Para la resolución de fuerza, el suavizado del potencial gravitacional de Plummer se fija en $\varepsilon=5 h^{-1} \mathrm{kpc}$ en unidades físicas a redshift $z \leq 5$, mientras que se cambia a unidades comóviles a redshifts más altos, adoptando el valor $\epsilon=30 h^{-1} \mathrm{kpc}$. La figura 2.1 muestra la distribución de gas de una simulación típica de la cual se extraen los cúmulos para ser resimulados. Los cúmulos $\mathrm{C} 15$ y C14 son mostrados en las figuras 2.2 y 2.3 , respectivamente.

Aunque las simulaciones son hidrodinámicas, sólo utilizamos la información cinemática provista por las partículas de materia oscura para identificar los halos de materia oscura. Con este propósito, consideramos que las partículas de materia oscura tienen sus masas incrementadas a su valor original, ya que el gas fue introducido en la región de alta resolución separando cada partícula en una de gas y otra de materia oscura. Los halos de materia oscura son identificados como grupos de partículas virializadas mediante el algoritmo "friends-of-friends" (FOF). El algortimo SUBFIND de (Springel et al., 2001) se aplica luego a estos grupos identificados por el FOFpara encontrar subestructuras de materia oscura ligadas dentro de los halos, a los que denominamos subhalos de materia oscura. Estos subhalos son los sitios de formación de galaxias. Extrayendo de todas las simulaciones la información sobre los halos y subhalos, se contruye el árbol de fusiones, para lo cual se tienen en cuenta los subhalos con 10 o más partículas ligadas, ya que grupos con menos partículas resultan dinámicamente inestables (Kauffmann et al., 1999). Con los árboles de fusiones podemos conocer la historia de formación de los halos y sub- 
halos a lo largo de la evolución del Universo. Cada simulación tiene 92 salidas separadas por un intervalo de tiempo de $\sim 200 \mathrm{Myr}$, comenzando en redshift $z \sim 60$ hasta $z=0$.

\subsection{Modelo Semianalítico SAG: física de los bariones o "gastrofísi- ca"}

Durante su evolución, las galaxies pueden adquirir masa estelar por formación de estrellas a partir del gas frío disponible, tanto en un modo pasivo o quiescent, o mediante brotes estelares disparados por inestabilidades de disco y/o fusiones de galaxias (mergers). Nos referimos al primer modo como "modo pasivo", para diferenciarlo de la SF más violenta que tiene lugar durante los brotes estelares. Durante las fusiones, las galaxias también pueden aumentar su masa debido a la acreción de los sistemas satélites. El contenido de gas frío de las galaxias proviene de los flujos de gas frío o cooling flows generados a partir del enfriamieto radiativo del gas caliente contenido en el halo de materia oscura en el que reside la galaxia; el gas frío acretado pasa a formar parte del disco galáctico. Subsecuentemente, la masa de la galaxia puede ser reducida debido al reciclado producido por vientos estelares y explosiones de supernovas. Estas últimas también reducen el gas frío disponible para la posterior formación estelar a través de efectos de retroalimentación (feedback) de energía. La actividad de núcleos galácticos activos (AGN, según su sigla en inglés, Active Galactic Nuclei) inyecta energía en la fase caliente y reduce el flujo de gas frío recibido por la galaxia, regulando así el proceso de formación estelar. La tasa de enfriamiento del gas caliente no sólo depende de la temperatura sino también del contenido de metales que son aportados por las supernovas y los vientos estelares. La compleja combinación de estos procesos se ve reflejada en las propiedades de las galaxias, tales como metalicidades y luminosidades. Todos estos procesos pueden ser tenidos en cuenta en un modelo semianalítico de formación de galaxias. La principal ventaja de esta herramienta numérica con respecto a las simulaciones de $N$ - cuerpos hidrodinámicas completamente autoconsistentes reside en el hecho de que los modelos semianalíticos permiten alcanzar un rango dinámico más grande a un costo computacional mucho más bajo.

El modelo semianalítico de formación de galaxias SAG usado en este trabajo está basado en el código descripto en Lagos et al. (2008). Los procesos físicos que se consideran en este modelo comprenden enfriamiento radiativo del gas caliente del halo, formación estelar en modo pasivo y en brotes estelares estelares causados por inestabilidades de disco y/o fusiones galácticas, y feedback por explosiones de supernova y por la acción de AGNs, entre otros aspectos. El modelado del proceso de feedback de AGN corresponde a la mejora implementada en el trabajo de Lagos et al. (2008), el cual se basa en el modelo semianalítico presentado por Cora (2006). Este último simula el resto de los procesos antes mencionados siguiendo las propuestas de Springel et al. (2001), quienes cedieron la versión original del código sobre la que se continuó trabajando. El modelo de Cora (2006) introduce una mejora importante con respecto al trazado de la circulación de metales entre diferentes componentes bariónicas, esto es, gas caliente y difuso, gas frío y estrellas, teniendo en cuenta la evolución de la masa de los diferentes elementos químicos. La versión del modelo semianalítico SAG usada en este trabajo implementa además un detallado cálculo del radio de escala de los discos galácticos, descripto en Tecce et al. (2010).

A continuación se describe el modelado de los procesos físicos incluidos en SAG . Debido a que los mismos no son del todo bien comprendidos, se utilizan parámetros libres que regulan el efecto de cada uno de ellos y que luego se ajustan, con el fin de reproducir diferentes relaciones observadas que que involucran propiedades de galaxias locales y a alto redshift. 


\subsubsection{Gas caliente, enfriamiento radiativo, formación estelar y feedback por su- pernovas}

De acuerdo a la información provista por los árboles de fusiones, se calcula la masa de gas caliente del halo de materia oscura para cada salida de la simulación de $N$-cuerpos en las cuales se aplica el modelo semianalítico. Debido a que el gas caliente tiene inicialmente la misma distribución espacial que la materia oscura, la masa virial se ve afectada por los procesos evolutivos de agregación jerárquica y cambia de un paso al siguiente. Cuando una fracción del gas caliente se enfría, los procesos de formación estelar comienzan a partir del gas frío disponible. La masa del gas caliente contenida dentro de cada halo FOF está dada por la expresión

$$
M_{\mathrm{hot}}=f_{b} M_{\mathrm{vir}}-\sum_{i=1}^{N_{\mathrm{FOF}}}\left(M_{\mathrm{Stellar}, i}+M_{\mathrm{ColdGas}, i}+M_{\mathrm{BH}, i}\right)
$$

siendo $M_{\text {Stellar }}$ la masa estelar, $M_{\text {ColdGas }}$ la masa de gas frío y $M_{\mathrm{BH}}$ la masa de agujeros negros centrales, para cada una de las $N_{\mathrm{FOF}}$ galaxias de los grupos FOF considerados en cada paso de la simulación. El factor $f_{b}$ es la fracción bariónica. La masa virial está dada por $M_{\mathrm{vir}}=100 H^{2} R_{v i r}^{3} / G$. Esta es la masa encerrada en un radio virial $R_{\mathrm{vir}}$, que se define como el radio de una esfera con densidad media $200 \rho$ crit, centrada en la partícula más masiva del grupo. Aquí, $\rho$ crit es la densidad crítica y la velocidad virial está dada por $V_{\mathrm{vir}}^{2}=G M_{\mathrm{vir}} / R_{\mathrm{vir}}$.

Se considera que el gas caliente tiene una distribución de tipo esfera isoterma. La masa de gas que se enfría en cada paso de la simulación, $M_{\text {cool }}$, está dada por la tasa de enfriamiento

$$
\frac{\mathrm{d} M_{\text {cool }}}{\mathrm{d} t}=4 \pi \rho_{g} r_{\text {cool }}^{2} \frac{\mathrm{d} r_{\text {cool }}}{\mathrm{d} t}
$$

donde $r_{\text {cool }}$ es el radio de enfriamiento. Esta cantidad se define como el radio dentro del cual el gas tiene un tiempo de enfriamiento menor a su tiempo dinámico. A su vez, el tiempo de enfriamiento local se define como el cociente entre el contenido específico de energía del gas y la tasa de enfriamiento por unidad de volumen. Esta última cantidad, $\Lambda(T, Z)$, depende fuertemente de la abundancia química $Z$ del gas y de la temperatura virial del halo. En el cálculo del tiempo de enfriamiento del gas, se emplean funciones de enfriamiento dependientes de la metalicidad, dadas por Sutherland \& Dopita (1993).

Cuando una galaxia que se convierte en satélite de un grupo o cúmulo sufre una remoción de todo el gas caliente que se encuentra atrapado en su pozo de potencial. A este proceso se lo llama "estrangulación" (Larson et al., 1980; Balogh et al., 2000; Kawata \& Mulchaey, 2008; Prescott et. al, 2011). En este escenario, la masa de gas y de metales contenidos en la fase de gas caliente de las galaxias satélites pasan al reservorio de gas caliente de la galaxia central del halo correspondiente.

En SAG, como en otros modelos semianalíticos, el enfriamiento radiativo sólo tiene lugar en las galaxias centrales del halo FOF . El enfriamiento da lugar a la formación de estrellas según una tasa dada por

$$
\frac{\mathrm{d} M_{\text {stars }}}{\mathrm{d} t}=\alpha \frac{M_{\text {ColdGas }}}{t_{\mathrm{dyn}}},
$$

donde $\alpha$ es un parámetro adimensional con el que se regula la eficiencia de formación estelar y $t_{\text {dyn }}=R_{\text {disc }} / V_{\text {disc }}$ es el tiempo dinámico de la galaxia, en cuya definición intervienen el radio del disco galáctico y su velocidad circular. La velocidad del disco, $V_{\text {disc }}$ se aproxima considerando que es un 25 por ciento más grande que la velocidad virial $V_{\text {vir }}$ definida más arriba. 
En el modelo SAG adoptamos la eficiencia de formación estelar variable introducida por De Lucia et al. (2004), que depende de propiedades del halo de Dark Matter (DM),

$$
\alpha\left(V_{\mathrm{vir}}\right)=\alpha_{0}\left(\frac{V_{\mathrm{vir}}}{220 \mathrm{~km} \mathrm{~s}^{-1}}\right)^{n}
$$

donde $\alpha_{0}$ y $n$ son dos parámetros libres. Cada evento de formación estelar genera una masa $\Delta M_{\text {star }}=M_{\text {star }} \Delta t_{\text {out }} / N_{\text {steps }}$, donde $N_{\text {steps }}=50$ son subdivisiones del paso de la simulación, de igual duración, utilizadas para integrar las ecuaciones diferenciales que describen la física de los bariones, y $t_{\text {out }}$ es el intervalo entre dos salidas sucesivas de la simulación.

En cada evento de formación estelar se produce un cierto número $\eta_{\mathrm{CC}}$ de supernovas de tipo colapso de núcleo (CCSN, según sus siglas en inglés, core-collapse supernovae) de tipos Ib/c y II, que se originan a partir de estrellas con masas mayores que $8 M_{\odot}$; esta cantidad depende de la función inicial de masa (IMF) adoptada. La IMF da la distribución del número de estrellas nacidas en un determinado rango de masas. En SAG, se utiliza la IMF propuesta por Salpeter en 1955 cuya forma funcional es

$$
N(m) d m \propto m^{-2,35} d m
$$

Al normalizar esta IMF entre 0,1 y $100 M_{\odot}$, el número de supernovas por cada masa solar de estrellas formadas es $\eta_{\mathrm{CC}}=6,3 \times 10^{-3} M_{\odot}^{-1}$. Cada CCSN libera una energía $E_{\mathrm{CC}}=1,2 \times 10^{51} \mathrm{erg} \mathrm{s}^{-1}$ (Woosely \& Weaver, 1995). Esta energía liberada calienta nuevamente parte del gas frío de la galaxia, en una cantidad dada por

$$
\Delta M_{\text {reheat }}=\frac{4}{3} \epsilon \frac{\eta_{\mathrm{CC}} E_{\mathrm{CC}}}{V_{\mathrm{vir}}^{2}} \Delta M_{*}
$$

donde $\epsilon$ es un parámetro adimensional que regula la eficiencia de feedback por CCSN. El destino de este gas recalentado puede modelarse. Si la fase caliente se mantiene dentro del halo de DM anfitrión, estamos en un "esquema de retención", si abandona el halo es un "esquema de eyección". Más detalles y otros modelados posibles pueden verse en el trabajo de De Lucia et al. (2004).

\subsubsection{Enriquecimiento químico}

En el modelo SAG, el gas primordial del cual se forman las estrellas está compuesto por un 76 por ciento de hidrógeno y un 24 por ciento de helio. A medida que la evolución estelar tiene lugar, las estrellas contaminan las fases fría y caliente del gas con metales eyectados en procesos evolutivos tales como pérdidas de masa por vientos estelares y explosiones de supernova. Este enriquecimiento químico afecta las tasas de enfriamiento del gas caliente de la galaxia, sensibles a la metalicidad. La tasa de enfriamiento del gas condiciona, a su vez, la tasa de formación de estrellas que tiene lugar a partir del gas frío disponible. Las tasas de pérdida de masa y la contribución de metales debidas a la evolución estelar dependen de los rangos de masa de las estrellas involucradas. Para estrellas de masas bajas e intermedias $\left(0,8 M_{\odot} \leq M \leq 5-8 M_{\odot}\right)$, se tienen en cuenta los resultados de los modelos de Marigo (2001); para el resto de las masas estelares $\left(0,8-5 M_{\odot} \leq M \leq 100 M_{\odot}\right)$, se adoptan los yields estelares dados por Portinari et al. (1998).

Las estrellas más masivas, dan lugar a explosiones de supernovas core collapse (SNCC), principales productoras de los elementos $\alpha$. Por otra parte, las supernovas tipo Ia contribuyen al enriquecimiento químico del medio interestelar (ISM, según siglas en inglés, 
Interstellar Medium) con elevada producción de hierro $\left(\sim 0,6 M_{\odot}\right)$. Adoptamos la formulación de nucleosíntesis propuesta por Iwamoto et al. (1999), para modelar la contribución química de estos eventos. En nuestros cálculos, seguimos la producción de 8 elementos químicos: $\mathrm{H},{ }^{4} \mathrm{He},{ }^{12} \mathrm{C},{ }^{14} \mathrm{~N},{ }^{16} \mathrm{O},{ }^{24} \mathrm{Mg},{ }^{28} \mathrm{Si} \mathrm{y}{ }^{56} \mathrm{Fe}$, generados por las estrellas con masas distribuidas en 27 rangos, desde $0,8 M_{\odot} \leq M \leq 100 M_{\odot}$. Se utilizan los tiempos de vida estelares dados por Padovani \& Matteucci (1993) para estimar la escala temporal de retorno a las distintas fases del ISM de los metales sintetizados y eyectados por todas las posibles fuentes. Los detalles de este modelo de enriquecimiento químico puede verse en el trabajo de Cora (2006).

\subsubsection{Inestabilidades de disco}

Se considera que los discos galácticos se forman a partir del enfriamiento del gas caliente contenido en los halos de materia oscura. Las fuerzas de marea imparten un momento angular a todo el material, incluyendo al gas, de manera que el gas frío, sin presión de sustentación, naturalmente se acomoda en una configuración de disco. Debido a que las galaxias espirales típicamente tienen discos con una distribución superficial de brillo de tipo exponencial, es común modelar al disco de las galaxias con una distribución superficial de la forma

$$
\Sigma(R)=\Sigma_{0} \exp \left(-R / R_{\mathrm{d}}\right),
$$

donde $R_{\mathrm{d}}$ es el radio de escala del disco, y la densidad superficial $\Sigma_{0}$ se calcula de acuerdo a la masa total del disco (Binney \& Tremaine, 1987).

Cuando un disco es lo suficientemente masivo como para que su autogravedad sea dominante, se torna inestable frente a pequeñas perturbaciones generadas por satélites menores o subestructuras de materia oscura. Para modelar esta inestabilidad del disco, adoptamos el criterio propuesto por Cole et al. (2000) que define la cantidad

$$
\varepsilon=\frac{V_{\mathrm{disc}}}{\left(G M_{\mathrm{disc}} / R_{\mathrm{d}}\right)^{1 / 2}} .
$$

Si $\varepsilon<\varepsilon_{\text {disc }}$, entonces el disco se vuelve inestable, siendo $\varepsilon_{\text {disc }}$ uno de los parámetros libres

del modelo que adopta valores cercanos a la unidad. Este mecanismo de inestabilidad de disco es capaz de producir galaxias elípticas; todo el gas y todas las estrellas del disco se transfieren al bulbo y, adicionalmente, el gas se consume en un brote de formación estelar que también contribuye a aumentar la masa del bulbo.

\subsubsection{Fusiones de galaxias}

En un escenario jerárquico de formación de estructuras, las fusiones entre galaxias son una consecuencia natural de las fusiones entre los halos de materia oscura en los que éstas residen. Las fusiones juegan un rol preponderante en la determinación de propiedades de las galaxias tales como la masa y la morfología. El catálogo de galaxias que utilizamos en este trabajo se construye aplicando el modelo seminanalítico a los árboles de fusión de los subhalos de materia oscura, extraídos de las simulaciones de cúmulos. Luego de la identificación de las subestructuras de materia oscura dentro de los grupos FOF, el subhalo más grande del grupo FOF será el que albergue a la galaxia central del grupo. La misma se localiza en la posición de la partícula del grupo más ligada gravitacionalmente. Estas galaxias se denominan "galaxias centrales" o "galaxias tipo 0". Las galaxias centrales de otros subhalos más pequeños contenidos dentro del mismo grupo FOF se llaman "galaxias del halo" o "galaxias tipo 1". Los subhalos que albergan a estas galaxias permanecen intactos luego de caer dentro de estructuras mayores. Existe un tercer grupo 
de galaxias llamadas "galaxias satélites" o "galaxias de tipo 2", que son aquéllas que se generan cuando dos subhalos se fusionan y la galaxia central del subhalo más pequeño pasa a ser satélite del subhalo remanente. Estas galaxias quedan huérfanas de su subhalo y se fusionan, eventualmente, con la galaxia central del subhalo remanente luego de que haya transcurrido un tiempo dado por la escala de tiempo de fricción dinámica (Binney \& Tremaine, 2008).

Las fusiones de galaxias se clasifican como fusiones mayores o menores según el valor del cociente entre las masas bariónicas, $M^{\text {sat }}$ y $M^{\text {central }}$, de la galaxia satélite acretada y la galaxia central; la masa bariónica es la suma de la masa de estrellas y la masa de gas de la galaxia. De este modo resulta definido el cociente

$$
f_{\text {merge }}=M^{\text {sat }} / M^{\text {central }} .
$$

Una fusión es "mayor" cuando el cociente $f_{\text {merge }}>0,3$. En caso contrario, se considera que tenemos una fusión "menor".

En las fusiones mayores, todas las estrellas presentes en las galaxias colisionantes se acomodan en un esferoide. La perturbación provocada por la fusión conduce al gas frío hacia el bulbo del remanente y lo comprime, dando lugar a un brote de formación estelar que consume todo el gas. De este modo, las fusiones mayores generan galaxias elípticas.

En las fusiones menores, todas las estrellas de la galaxia satélite se agregan al bulbo del remanente, mientras que el disco de estrellas de la galaxia más grande permanece sin alteraciones. La presencia de un brote estelar durante una fusión menor depende de la fracción de masa de gas presente en la galaxia central, dada por el cociente

$$
f_{\text {ColdGas }}^{\text {central }}=\frac{M_{\text {ColdGas }}^{\text {central }}}{M_{\text {disc }}^{\text {central }}}
$$

donde $M_{\text {disc }}=M_{\text {Stellar }}-M_{\text {Bulge }}+M_{\text {ColdGas }}$ es la masa del disco, definida en términos de la masa de la componente estelar de la galaxia, $M_{\text {stellar}}$, de la masa del bulbo (formado sólo por estrellas), $M_{\text {Bulge }}$, y la masa de gas frío de la galaxia, $M_{\text {ColdGas. Si } f_{\text {ColdGas }}^{\text {central }}>}$ $f_{\text {gas,minor }}$, todo el gas frío de las galaxias colisionantes se consume en un brote estelar y las estrellas formadas se agregan al bulbo del remanente. Este parámetro umbral, $f_{\text {gas,minor }}$, es un parámetro libre motivado por las simulaciones de Hernquist \& Mihos (1995), quienes hallan que las galaxias de disco ricas en gas tienen más probabilidades de generar un brote estelar bajo la interacción con pequeños satélites (Malbon et al., 2007). Las fusiones menores en las cuales se producen brotes estelares se llaman "fusiones menores húmedas" (minor wet mergers). En estos eventos, la masa estelar de la galaxia central se incrementa gracias a que suma las contribuciones estelares de las nuevas estrellas producidas durante la fusión, más las estrellas de la galaxia satélite acretada. En cambio, si $f_{\text {ColdGas }}^{\text {central }}<$ $f_{\text {gas,minor }}$, no se produce formación estelar durante la fusión menor y, por lo tanto, se habla de "fusiones menores secas" (minor dry mergers). Más aun, los brotes estelares no tienen lugar si la galaxia satélite es muy pequeña comparada con la galaxia central con la que se fusiona. Es decir, si $f_{\text {merge }}<f_{\text {burst }}$, con $f_{\text {burst }}=0.05$, no se producirá un brote estelar en la coalescencia de las galaxias, sin importar la cantidad de gas disponible en la galaxia central. Este tipo de evento también es considerado como una fusión menor seca.

Para el propósito del presente estudio, clasificamos también a las fusiones mayores en "secas" y "húmedas". Esta clasificación no afecta el funcionamiento del código SAG, y se realiza con el sólo objeto de identificar los procesos responsables de la evolución galáctica. Una vez que se ha identificado en el modelo una fusión mayor, la clasificamos como húmeda o seca, dependiendo de la fracción de gas del disco del remanente. Luego,

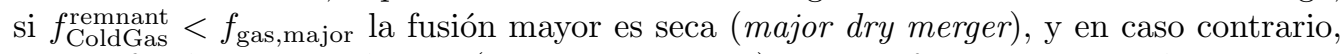
es una fusión mayor húmeda (major wet merger), siendo $f_{\text {gas,major }}$ un parámetro libre del modelo. 


\subsubsection{Feedback por Núcleos Galácticos Activos}

Un aspecto importante de las inestabilidades de disco e interacciones entre galaxias, especialmente, de las fusiones húmedas, es que pueden ser detonantes de brotes estelares y de la actividad de los AGN. El gas de los discos galácticos, a causa de la perturbación inducida por la fusión, pierde presión de sustentabilidad y fluye hacia el agujero negro central de la galaxia, motor del AGN, aumentando la actividad de éste. Existe una creciente evidencia observacional y teórica sobre la importancia de los AGN en la evolución galáctica. Los AGN son productores de grandes cantidades de energía que se inyectan en la galaxia a través de "jets" o flujos de material muy colimado. Se los considera responsables de reducir, o incluso, suprimir los flujos de gas frío de las galaxias residentes en halos masivos, afectando la formación estelar, y por ende, las masas estelares, las luminosidades y los colores (e.g. Croton et al. 2006; Malbon et al. 2007; Lagos et al. 2008). Este hecho permite reducir el número de galaxias en el extremo brillante de la función de luminosidad, permitiendo obtener un mejor acuerdo con los datos observados. Lograr esta mejora ha sido uno de los principales motivos de la inclusión de feedback de AGN en los modelos de formación de galaxias.

La conexión entre la evolución de las galaxias y los AGN se pone de manifiesto a través de correlaciones entre el agujero negro central y las propiedades de la galaxia en la que éste reside. Se han hallado correlaciones de la masa del agujero central de las galaxias con la masa del bulbo de las galaxias (Marconi \& Hunt, 2003; Haring \& Rix, 2004), la luminosidad en las bandas $K-, B-$ y $V-$ (Marconi \& Hunt 2003) y la velocidad de dispersión de las estrellas en el bulbo (Ferrarese \& Merrit, 2000).

En el modelo SAG, los AGN se producen a través del crecimiento de los agujeros negros centrales (ver Lagos et al., 2008), que tiene lugar durante la acreción de gas frío en el proceso de enfriamiento del gas (modo radio), y como consecuencia de la caída de gas hacia el centro galáctico a raíz de los eventos de fusión o de inestabilidad de disco (modo quasar). En los eventos de fusión consideramos que los agujeros negros centrales se fusionan instantáneamente, y la masa del agujero negro de la galaxia satélite se agrega a la masa del agujero negro de la galaxia central. Durante los eventos que dan lugar a brotes estelares, la masa de gas acretada por el agujero negro es proporcional a la masa de gas presente en la galaxia

$$
\Delta M_{\mathrm{BH}, \text { merger }}=f_{\mathrm{BH}} \frac{M_{\text {sat }}}{M_{\text {central }}} \frac{M_{\text {ColdGas }}}{1+\left(200 \mathrm{~km} \mathrm{~s}^{-1} / V_{\text {vir }}\right)^{2}},
$$

donde $M_{\text {ColdGas }}$ es la masa de gas de la galaxia central después que se ha agregado la masa de la galaxia satélite, es decir, la masa del gas del remanente. El crecimiento del agujero negro a través de la inestabilidad del disco también está representado en esta ecuación si se cambia el cociente $M_{\text {sat }} / M_{\text {central }}$ por 1, ya que este proceso es una interacción dinámica propia de la galaxia. El valor del parámetro libre $f_{\mathrm{BH}}$ se ajusta de manera de reproducir la relación observada entre masa de bulbo y masa del agujero negro. El otro modo de crecimiento de los agujeros negros (modo radio) tiene lugar cuando se ha establecido un halo de gas caliente alrededor de la galaxia anfitriona a partir del cual se producen los flujos de gas frío. Se considera que tasa de acreción de masa del agujero negro es continua y se describe mediante el siguiente modelo fenomenológico

$$
\dot{M_{\mathrm{BH}}}=\kappa_{\mathrm{AGN}} \frac{M_{\mathrm{BH}}}{10^{8} M_{\odot}} \frac{f_{\mathrm{hot}}}{0,1}\left(\frac{V_{\mathrm{vir}}}{200 \mathrm{~km} \mathrm{~s}^{-1}}\right)^{3} .
$$

donde $M_{\mathrm{BH}}$ es la masa del agujero negro, $f_{\text {hot }}=M_{\mathrm{hot}} / M_{v i r}$ es la fracción de masa total del halo en forma de gas caliente, y $\kappa_{\mathrm{AGN}}$ es un parámetro libre. Cabe destacar que debido al proceso de estrangulación, sufrido por las galaxias satélites, las mismas no disponen de 
un reservorio de gas caliente como las galaxias centrales, por lo que sus agujeros negros no experimentan este tipo de crecimiento.

A través de la tasa de acreción del agujero negro, puede calcularse su luminosidad en términos de $\eta_{\mathrm{BH}}$, la eficiencia estándar de producción de energía cerca del horizonde de eventos del agujero negro, por medio de la expresión

$$
L_{\mathrm{BH}}=\eta_{\mathrm{BH}} \dot{M_{\mathrm{BH}}} c^{2},
$$

donde $c$ es la velocidad de la luz. El calentamiento mecánico generado por la acreción del agujero negro (la luminosidad $L_{\mathrm{BH}}$ ) inyecta suficiente energía en el medio cincundante como para regular el enfriamiento del gas, reduciendo e incluso detiendo completamente los flujos de gas frío recibidos por la galaxia.

\subsubsection{Modelado de discos galácticos}

El modelo SAG utilizado en este trabajo incluye un detallado métodqo para calcular el tamaño de los discos de las galaxias desarrollado en el trabajo de Tecce et al. (2010), el cual sigue los lineamientos presentados en (2009). El procedimiento adoptado considera que el proceso de formación de discos sólo tiene lugar en las galaxias centrales. Cuando una galaxia se convierte en satélite, la acreción de gas frío se detiene a causa de la estrangulación, de manera que su disco no puede continuar creciendo. Entonces, el valor del radio de escala $R_{\mathrm{d}}$ queda fijado en el valor que tenía la última vez que la galaxia en cuestión fue identificada como galaxia central. Para cada paso de tiempo de la simulación y para cada galaxia que fue identificada como central, se determinan valores característicos como la masa de estrellas y gas frío de la galaxia, y la masa virial, concentración y parámetro de spin de su halo FOF anfitrión. A partir de estos valores, se calcula el valor de $R_{\mathrm{d}}$ en un dado redshift $z$, mediante un esquema de interpolaciones polinomiales sucesivas (ver Tecce et al. 2010).

\subsubsection{Propiedades espectro fotométricas de las galaxias}

Para estimar las luminosidades y colores de las galaxias se deben calcular sus propiedades espectro fotométricas. La luz recibida proveniente de las galaxias está compuesta por la luz de cientos de miles de estrellas $\left(10^{11}-10^{12}\right)$, cada una con cierta edad, masa y composición química. A efectos de poder interpretar lo que vemos y vincular la luz de las galaxias con las poblaciones estelares que las componen, y con la formación y evolución de las mismas, adoptamos una técnica que considera la caracterización de la fuente emisora mediante su distribución espectral de energía (SED, según siglas en inglés, Spectral Energy Distribution). Esta distribución espectral es una función $F \lambda$ que nos da la energía total de los fotones emitidos por la fuente en cierto rango de longitudes de onda $(\lambda, \lambda+$ $\mathrm{d} \lambda)$.

Interpretar la SED requiere conocer la emisión de cada una de las estrellas que forman las poblaciones estelares de una galaxia. En esto hay una ventaja, pues si bien la historia de formación de las galaxias continua siendo objeto de debate y estudio, la teoría de evolución estelar es mejor entendida. Con los modelos vigentes de evolución estelar se puede calcular con gran precisión la evolución de la luminosidad, color y el espectro integrado de una estrella a lo largo de toda su vida, dada su masa inicial y composición química primordial. Además, se pueden estimar las tasas de pérdida de masa, energía y metales, cantidades que son devueltas por las estrellas al medio interestelar. Estos son los ingredientes fundamentales del enriquecimiento químico de las poblaciones estelares subsiguientes, y por ende, de la evolución química de las galaxias.

Para reproducir el espectro de las poblaciones estelares presentes en una galaxia, se consideran modelos evolutivos de síntesis de poblaciones estelares. En estos modelos, los 
parámetros a ajustar son la tasa de formación estelar (SFR, según siglas en inglés, Star Formation Rate), y la IMF. En algunos casos, también se requiere conocer la tasa de enriquecimiento químico. Los modelos de síntesis de poblaciones hacen hipótesis sobre la evolución de estos parámetros con el tiempo y calculan la distribución, dependiente de la edad, de las estrellas en el diagrama Hertzsprung-Russell (HR). Una reproducción de este diagrama puede verse en la figura 6.5. A partir de las posiciones en el diagrama HR de estrellas de diferentes masas y composiciones químicas pero de la misma edad, se obtienen las "isocronas". Los modelos evolutivos de síntesis de poblaciones hacen uso de estas isocronas para estimar la evolución del espectro integrado de una población estelar. El método de síntesis de isocronas se basa en que las poblaciones estelares, cualquiera sea su SFR, se pueden describir como una sucesión de brotes estelares instantáneos, usualmente llamados "poblaciones estelares simples" (SSPs, según siglas en inglés, Simple Stellar Populations).

Suponiendo una IMF independiente del tiempo, la SED de una población estelar a tiempo $t$, caracterizada por cierta SFR, $\psi(t)$, y enriquecimiento químico $\zeta(t)$, está dada por

$$
F_{\lambda}(t)=\int_{0}^{t} \psi\left(t-t^{\prime}\right) S_{\lambda}\left[t^{\prime}, \zeta\left(t-t^{\prime}\right)\right] d t^{\prime}
$$

donde la función $S_{\lambda}\left(t^{\prime}, \zeta\left(t-t^{\prime}\right)\right)$ es la potencia radiada por una SSP de edad $t^{\prime}$ y metalicidad $\zeta\left(t-t^{\prime}\right)$ por unidad de longitud de onda $\lambda$, y por unidad de masa. Esta función, $S_{\lambda}$ se obtiene sumando el espectro de todas las estrellas que definen la isocrona de la SSP de edad $t^{\prime}$, con la metalicidad $\zeta\left(t-t^{\prime}\right)$.

El segundo ingrediente de los modelos evolutivos de síntesis poblacional es la librería de espectros individuales que se utiliza para describir las propiedades de las estrellas en cualquier posición del diagrama HR. Existen dos enfoques diferentes para obtener estas librerías. El primero es observacional, donde se obtiene una librería mediante la muestra de estrellas del vecindario solar. Como consecuencia, la muestra no es completa en los rangos de masa y metalicidad. Para obtener el espectro de una estrella fuera de la muestra, se interpolan o extrapolan, según convenga, los datos de la muestra, introduciendo errores significativos para estrellas diferentes a las del vecindario solar. El otro enfoque consiste en intentar predecir por otros métodos la SED de las estrellas. Mediante modelos de atmósferas estelares que calculan la transferencia radiativa a través de la superficie de la atmósfera, se obtiene la intensidad específica de la radiación emergente de la superficie de la atmósfera de las estrellas, en un amplio rango de masas, metalicidades, temperaturas efectivas y gravedad superficial (Kurucz, 1992; Allard \& Hauschildt, 1995).

El modelo evolutivo de síntesis de población estelar utilizado en SAG, del cual se obtienen las magnitudes de las galaxias en distintas bandas del espectro, es el de Bruzual \& Charlot (2003, en adelante nos referiremos a este trabajo como BC03). En el mismo, se utilizan tres librerías de tracks evolutivos calculados por distintos modelos de evolución estelar en distintos rangos de metalicidad; éstos son Padova 1994, Padova 2000 y Geneva. A su vez, se hace uso de diferentes librerías de espectros estelares, siendo dos de ellas observacionales (STELIB y Pickles) y una teórica (BaSel).

Las librerías de tracks evolutivos no incluyen de manera consistente la evolución de estrellas en la rama asintótica de las gigantes, en su fase de pulsos térmicos (TPAGB, según siglas en inglés, Thermally Pulsating Asymptotic Giant Branch). Tampoco se incluye el espectro de estas estrellas en ninguna de las tres librerías espectrales. Esta fase evolutiva es especialmente complicada de modelar. En las librerías de BC03, se utilizan aproximaciones de los espectros y formulaciones observacionalmente motivadas de estas estrellas TP-AGB, basados en modelos y datos de la Galaxia.

BC03 estudian la evolución de los colores $(B-V)$, y $(V-K)$ y del cociente masa luminosidad $M / L_{V}$, de una SSP estándar, considerando diferentes rangos de metalicidad estelar, $Z=0,004, Z=0,02$ y $Z=0,05$. Concluyen que a edades fijas, el efecto principal del aumento de la metalicidad es enrojecer los colores y aumentar el cociente masa 


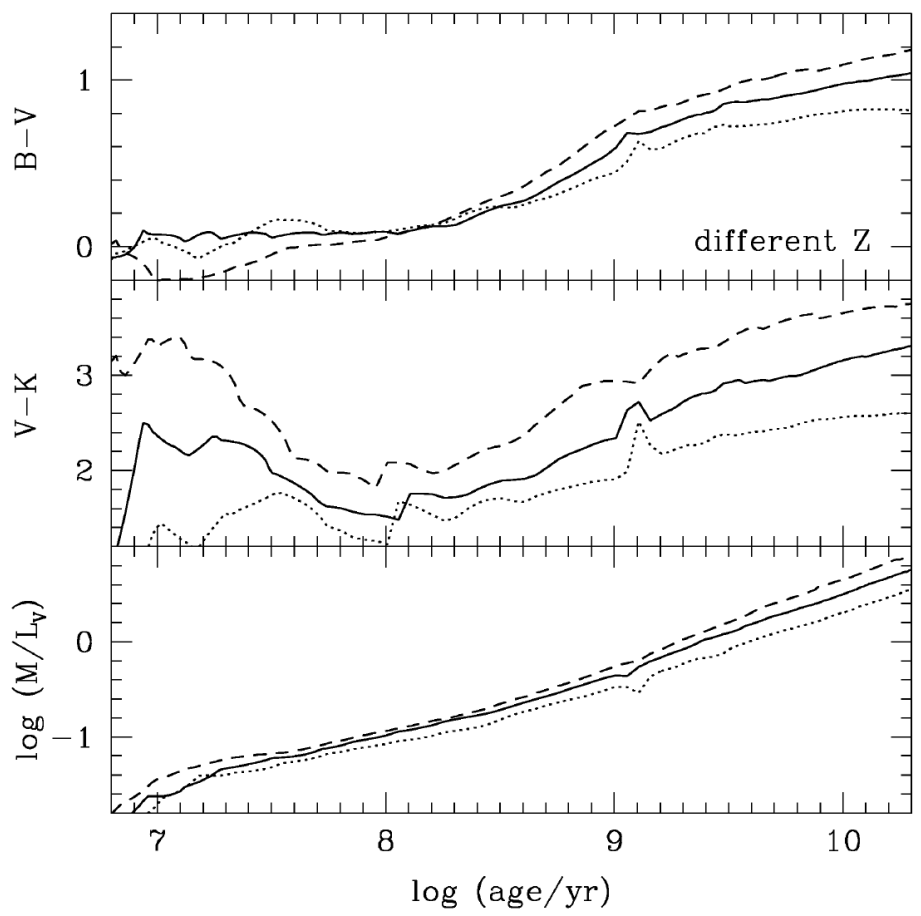

Figura 2.4: Evolución de los colores $B-V$ y $V-K$ y el cociente masa luminosidad $M / L_{V}$ de una SSP estándar, según las metalicidades $Z=0,004$ (línea punteada), $Z=0,02$ (línea sólida) y $Z=0,05$ (línea a trazos). Esta figura fue extraída del trabajo de Bruzual \& Charlot (2003) (figura 1). 
luminosidad $M / L_{V}$, tal como se aprecia de la figura 2.4.

A partir del uso del los modelos de síntesis de poblaciones estelares de $\mathrm{BC} 03$, el modelo SAG proporciona las magnitudes absolutas de las galaxias en el sistema de Johnson Morgan $(U, B, V, K, R, I)$ y las bandas $u, g, r, i, z$ del SDSS (Fukugita et al., 1995). La predicción final del espectro de las galaxias deberá incluir una formulación adecuada del efecto de atenuación por polvo interestelar.

\subsubsection{Extinción por polvo interestelar}

En el medio interestelar existe una componente de material sólido formado por granos de polvo. Los efectos de esta componente sobre la SED, (componente estelar, únicamente) no fueron evidenciados sino hasta 1930, cuando Robert Julius Trumpler mediante el estudio de cúmulos abiertos de la Galaxia, demostró de manera fehaciente la existencia de un medio interestelar capaz de atenuar fuertemente la luz proveniente de las estrellas. La gran opacidad del medio que compone el ISM puede apreciarse mediante el siguiente experimento mental: si comprimiésemos el medio ISM (densidad promedio, $\left.\sim 10^{-24} \mathrm{gr} / \mathrm{cm}^{3}\right)$ a la densidad típica del aire en La Tierra $\left(\sim 1,3 \times 10^{-3} \mathrm{gr} / \mathrm{cm}^{3}\right)$, hallaríamos que la visibilidad de los objetos que nos rodean está limitada aproximadamente a un metro de distancia.

Los mecanismos de formación de las partículas de polvo comprenden distintas etapas de la evolución estelar. Evidencias observacionales sugieren que a alto redshift $(z>4)$ los granos de polvo se producen mayormente en eventos de supernova CCSN (Maiolino et al., 2004), mientras que a bajo redshift la producción de los granos de polvo está localizada en las envolturas de estrellas en la rama asintótica de las gigantes (AGB, según sus siglas en inglés, Asymptotic Giant Branch). Luego, las partículas son distribuidas en el ISM mediante vientos estelares.

Los granos de polvo varian en tamaño, los mismos pueden ser granos de unos pocos micrones hasta moléculas formadas por 100 átomos. Su composición química está dada principalmente por silicatos, grafitos e hidrocarbonos políciclicos aromáticos (PAHs, según sus siglas en inglés, Polycyclic aromatic hydrocarbons. La presencia del polvo modifica la SED intrínseca de las galaxias debido a que la absorción y dispersión de la luz es mayor a longitudes de onda corta, en el rango de ultravioleta (UV): la energía absorvida por los granos de polvo es luego reemitida en el rango del infrarrojo (IR), produciendo un enrojecimiento del espectro de la fuente.

La atenuación de la intensidad de la fuente debida a la interacción con el polvo se describe mediante una ley exponencial. Esta ley vincula la intensidad intrínseca de la fuente, $I_{\lambda}^{0}$, en cierta longitud de onda $\lambda$, la intensidad observada en la línea de la visual luego de la interacción con el polvo, $I_{\lambda}$, y la profundidad óptica del medio, $\tau_{\lambda}$ : esta última contiene información sobre las propiedades del polvo a lo largo de la línea de la visual. Matemáticamente, la ley puede expresarse como

$$
I_{\lambda}=I_{\lambda}^{0} \exp \left(-\tau_{\lambda}\right) .
$$

La extinción efectiva $A_{\lambda}$, en cierta longitud de onda $\lambda$, puede definirse en función de las magnitudes de la siguiente manera

$$
A_{\lambda} \equiv m_{\lambda}-m_{\lambda_{0}}=-2,5 \log \left(\mathrm{L}_{\lambda} / \mathrm{L}_{\lambda_{0}}\right) .
$$

Generalmente, para nuestra Galaxia y otras galaxias del Grupo Local se establece una ley empírica de extinción, $k(\lambda)$, basada en observaciones directas 


$$
k(\lambda)=R_{V} \frac{A_{\lambda}}{A_{V}}
$$

donde $R_{V} \equiv A_{V} / E(B-V)$, siendo $E(B-V)=A_{B}-A_{V}$ el exceso de color medido entre las bandas $B$ y $V$, y $A_{V}$ es la extinción en la banda $V$, dada por la expresión 2.16. El cociente entre polvo y gas a lo largo de la línea de la visual, $\eta$, se expresa en términos de la opacidad $\tau_{V}$, y de la densidad columnar del gas neutro $N_{21}$ (en unidades $10^{21} \mathrm{~cm}^{-2}$ ), independientemente de la forma que adopte el hidrógeno, ya sea molécular $\left(\mathrm{H}_{2}\right)$ o átomico neutro $(\mathrm{HI})$. La expresión resulta

$$
\eta=\tau_{V} / N_{21} \sim 0,921 R_{V} E(B-V) / N_{21}
$$

Conociendo $k(\lambda), R_{V}$ y $\eta$, se puede determinar la atenuación por polvo $A_{\lambda}$, utilizando observaciones de la densidad columnar del hidrógeno neutro a lo largo de la línea de la visual. La ley de extinción, $k(\lambda)$, es diferente según las particularidades del polvo de cada galaxia. Para la Vía Láctea se obtiene un valor de $R_{V} \sim 3,1$, mientras que para la Nube Mayor de Magallanes resulta $R_{V} \sim 2,7$ (Cardelli et al., 1989).

En SAG, se modela la extinción por polvo solamente para los discos de las galaxias de la simulación, siguiendo una formulación observacionalmente motivada, sugerida por Kauffmann et al. (1999). La misma hace uso de una correlación observada por Wang \& Heckman (1996), quienes hallan una vinculación entre cantidades como la luminosidad de la galaxia y la profundidad óptica del polvo en un disco galáctico visto de frente. La expresión en la banda $B$ de dicha correlación adopta la forma

$$
\tau_{B}=(0,8 \pm 0,3)\left(\frac{L_{B}}{L_{B} *}\right)^{0,5}
$$

llamamos $L_{B}$ a la luminosidad intrínseca (no atenuada) de la galaxia en la banda $B$ y $L_{B} *$ a la luminosidad observada de una galaxia de magnitud $M_{B}=-19,6+5 \log (\mathrm{h})$.

Se utiliza el cociente $\tau_{\lambda} / \tau_{B}$ para calcular la extinción en otras bandas fotométricas, aplicando la curva de extinción de la Galaxia dada por Cardelli et al. (1989).

El modelado se completa proponiendo una dependencia de la atenuación con el ángulo de la observación. La geometría más simple supone un disco galáctico de capas delgadas planas y paralelas con una distribución uniforme de polvo y estrellas. La línea de la visual forma un ángulo $\theta$ con respecto a la inclinación del disco, que el modelo SAG asigna de manera aleatoria a cada galaxia. La extinción efectiva de la fuente expresada en magnitudes resulta

$$
A_{\lambda}=-2,5 \log \left(\frac{1-\exp \left(-\tau_{\lambda} \sec \theta\right)}{\tau_{\lambda} \sec \theta}\right)
$$

(Guideroni \& Rocca-Volmerange, 1987). Existen en la literatura diferentes soluciones analíticas para diversas configuraciones geométricas, como por ejemplo, el caso de un elipsoide oblado, estudiado por Devriendt et al. (1999).

\subsubsection{Calibración de SAG}

Los procesos físicos incluidos en SAG, descriptos a lo largo de esta sección, involucran parámetros libres que deben ser ajustados a fin de reproducir simultáneamente la mayor cantidad propiedades observadas de las galaxias. Algunas de las correlaciones con las 


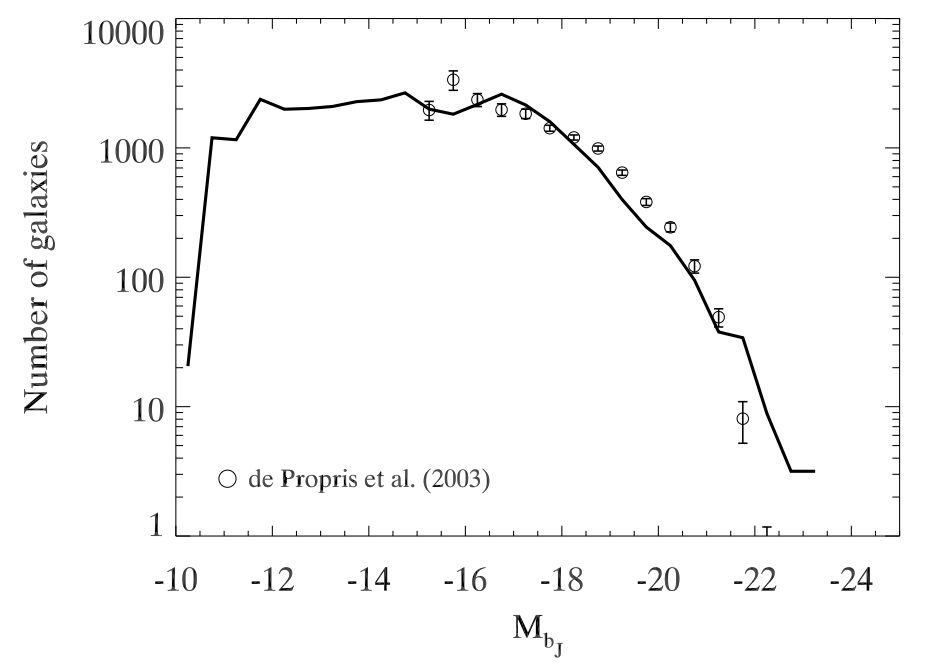

Figura 2.5: Función de luminosidad compuesta en la banda $b_{j}$ para todos los cúmulos simulados. Los símbolos con barra de error corresponden a observaciones de 60 cúmulos del Universo local de DePropris et al. (2003).

cuales se calibra el modelo son la función de luminosidad, las relaciones entre la masa del agujero negro central y el bulbo de la galaxia en la que reside, la relación color magnitud, la relación masa metalicidad, la relación luminosidad metalicidad, y la relación entre el tamaño y la luminosidad del disco galáctico, la función de luminosidad de los quásares, y las funciones de masa en diferentes redshifts, entre otras.

Los valores de los parámetros libres adoptados en este trabajo son: $\alpha_{0}=0,1$ y $n=$ 2,2 que intervienen en la definición de eficiencia variable de formación estelar, ecuación (2.4); $\epsilon=0,1$ para la eficiencia de feedback por supernovas, ecuación $(2.6) ; \varepsilon_{\text {disc }}=0,8$ para evaluar la inestabilidad de disco, ecuación (2.7); $f_{\text {gas,minor }}=0,6, f_{\text {burst }}=0,05 \mathrm{y}$ $f_{\text {gas,major }}=0,4$, para las fracciones que intervienen en la definición y caracterización de los distintos tipos de fusiones (ver sección 2.3.4); y $f_{\mathrm{BH}}=0,04$ y $K_{\mathrm{AGN}}=1 \times 10^{-3}$ para los factores que regulan el crecimiento del agujero negro, ecuaciones (2.11) y (2.12). Con respecto a los posibles valores del parámetro $f_{\text {gas,major }}$, discutiremos su relevancia en la interpretación de las propiedades de las galaxias de la RCM en el capítulo 4.

Las figuras 2.5 y 2.6 muestran, respectivamente, la función de luminosidad en la banda $b_{j}$ y la relación entre las masas del agujero negro y del bulbo, resultantes de aplicar el modelo SAG calibrado con los parámetros antes mencionados a las simulaciones de cúmulos consideradas en este trabajo. 


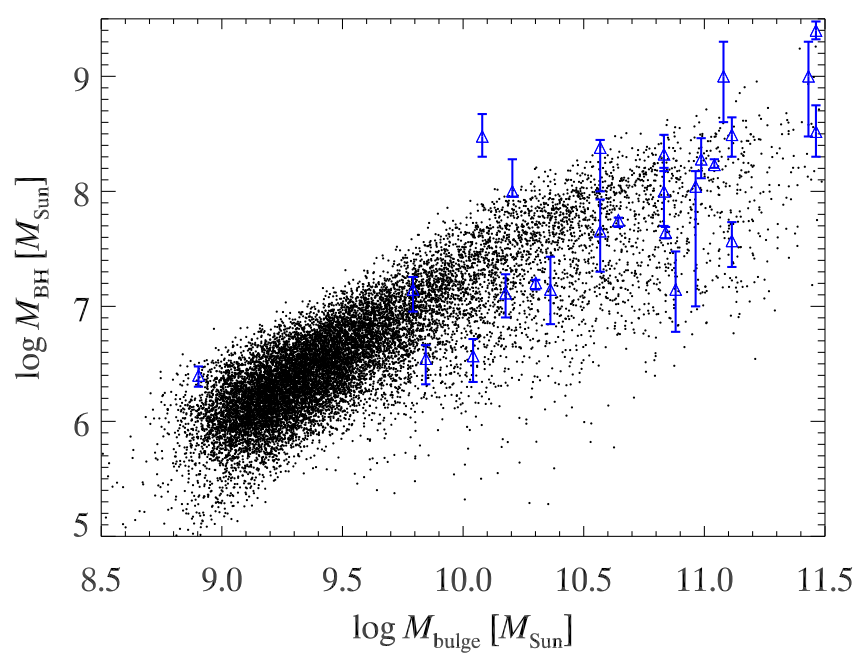

Figura 2.6: Relación entre las masas del agujero negro central y del bulbo de las galaxias para el cúmulo $C 15_{3}$ (g51). Los símbolos con barras de error son muestras observacionales de Haring \& Rix (2004). 


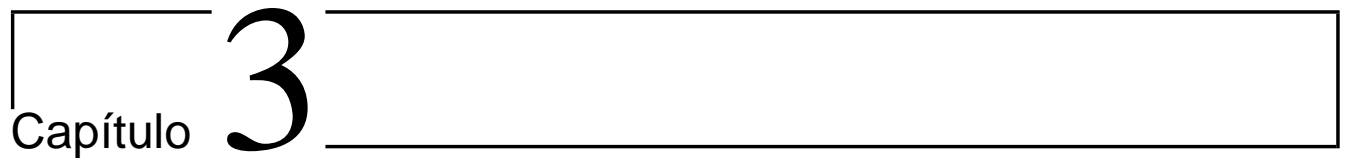

\section{La Relación Color Magnitud}

A partir de la aplicación del modelo semianalítico SAG calibrado para reproducir numerosas propiedades observadas de las galaxias, tanto locales como a alto redshift, se obtiene la RCM en distintas bandas fotométricas, utilizando diferentes criterios de selección de galaxias de tipo temprano. En este capítulo discutimos las características de las RCM resultantes. Además, analizamos las similitudes y diferencias con relaciones observadas. Con este propósito, se estiman las pendientes de rectas de ajustes a toda la relación y a sus extremos débil y brillante, y se comparan con las reportadas por estudios observacionales. Se analiza, además, la relación luminosidad metalicidad definida por las galaxias de la RCM simulada. Este análisis permite concluir que es factible hacer uso de las historias químicas de las galaxias generadas por el modelo para ayudar a entender las características de la RCM y las razones físicas que dieron lugar a las mismas.

\subsection{Criterios de selección de galaxias que forman la RCM modela- da}

El modelo semianalítico SAG, produce una población de galaxias que puede clasificarse de acuerdo a características tales como el tipo morfológico, la tasa de formación estelar específica, el color integrado o la metalicidad. Una vez conocida la distribución de galaxias en el diagrama color magnitud, seleccionamos a los miembros de la RCM de acuerdo a algún criterio, y estudiamos las características principales de las galaxias que la componen, así como las propiedades de la RCM misma. Existen en la literatura diversos criterios de clasificación de las galaxias en la RCM. Los estudios sobre la bimodalidad en los colores del diagrama color magnitud muestran que la distribución de galaxias presenta dos picos, uno rojo y otro en azul. Las galaxias distribuidas alrededor del primero son galaxias rojas, con baja formación estelar y tipos morfológicos más tempranos que Sa, mientras que alrededor del segundo pico se encuentran galaxias más tardías que las $\mathrm{Sb}$ con formación estelar activa. Esta bimodalidad en los colores se extiende hasta $z \sim 1$ (ver captítulo 1). Luego, las galaxias de la RCM pueden seleccionarse de acuerdo a los colores, la formación estelar o la morfología. Investigamos aquí la RCM resultante de aplicar distinos criterios de selección a las galaxias simuladas dadas por SAG, para esclarecer si existe una dependencia entre los diferentes criterios de selección y las características generales de la RCM. Si bien las magnitudes de las galaxias del modelo SAG están dadas en el sistema Johnson-Morgan, en esta sección mostramos las RCM en el sistema de magnitudes fotométricas de Washington $\left(C, T_{1}\right)$; elegimos este sistema por ser especialmente sensible a las metalicidades de las galaxias (ver sección 3.2). 


\subsubsection{Criterio de selección basado en la bimodalidad de la distribución de colo- res}

Primeramente, seleccionamos galaxias por color adoptando el criterio empírico dado por Bell et al. (2004), obtenido a partir de galaxias en el rango de redshift $0,2>z>1,1$ detectadas en el relevamiento COMBO-17 (Classifying Objects by Medium-Band Observations in 17 Filters). Estos autores hacen uso de la bimodalidad de colores en el diagrama color magnitud, que es observada hasta $z \approx 1$, y establecen una separación dependiente del redshift entre la secuencia roja y la azul. La distribución de colores de las galaxias se analiza en el plano $U-V$ versus $V$ debido a que el color $U-V$ abarca el quiebre en $4000 \AA$ resultando sensible a los cambios de edad y metalicidad de las poblaciones estelares de las galaxias. Los autores no tienen en cuenta las morfologías de las galaxias en la determinación de su criterio. Concretamente, galaxias más rojas que

$$
\langle U-V\rangle=1,15-0,31 z-0,08\left(M_{\mathrm{V}}-5 \operatorname{logh}+20\right)
$$

pertenecen a la secuencia roja y las restantes a la secuencia azul. Bell et al. (2004) otorgan validez a esta relación dentro del rango de redshift $0<z<1$.

Aunque el estudio de Bell et al. (2004) no está restringido a ambientes de cúmulos de galaxias, encontramos que su criterio es adecuado para seleccionar galaxias de la RCM en este tipo de ambientes, dada la uniformidad de la RCM en entornos de distinta densidad, es decir, cúmulos, grupos y campo (v.g López-Cruz et al., 2004; Hogg et al., 2004; McIntosh et al., 2005; Stott et al., 2009; Martínez et al., 2010). La pendiente y el punto de cero de la RCM de Bell et al. (2004) están en concordancia con las determinaciones previas obtenidas a partir de observaciones de cúmulos de galaxias en el universo local realizadas por Bower et al. (1992) y Terlevich et al. (2001), así como también con los valores determinados por Schweizer \& Seitzer (1992) para galaxias de campo E y S0. El punto cero de la RCM es consistente con el hallado para galaxias de tipo temprano pertenecientes a un gran rango de ambientes según se obtiene del Nearby Field Galaxy Survey (Jansen et al. 2000; McIntosh et al. 2004).

Procedemos entonces a aplicar el criterio de Bell et al. (2004) adoptando el valor $z=0$ para hallar la RCM de los cúmulos simulados en el universo local y realizar la posterior comparación con datos observacionales locales. A partir de los resultados de SAG, analizamos los cúmulos de galaxias más masivos que forman parte del set C15.

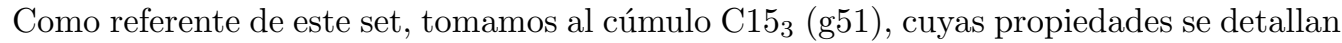
en el capítulo 2. En la figura 3.1 puede verse la RCM para el cúmulo $\mathrm{C} 15_{3}$ en el sistema de magnitudes fotométricas de Washington. Los símbolos de diferentes colores indican distintos rangos de metalicidad alcanzados por las galaxias. Podemos ver que las galaxias más luminosas de la RCM corresponden a los valores máximos de metalicidad $([\mathrm{Fe} / \mathrm{H}] \approx$ $0,43)$, mientras que las menos luminosas presentan valores menores de metalicidad, de manera que se hace evidente una relación del tipo "masa metalicidad". Esta relación es consistente con la idea de que las galaxies más luminosas, y por lo tanto más masivas, tienen profundos pozos de potencial gravitatorio que les permite retener los metales liberados por eventos de supernovas y vientos estelares, resultantes de la evolución de sus poblaciones estelares. En la figura también se muestran las rectas de ajuste a distintas secciones de la RCM y a la relación completa, realizados por la técnica de mínimos cuadrados. El detalle de estos ajustes y su significancia para este trabajo se discuten más adelante, en la sección 3.2 .

\subsubsection{Criterio de selección por tipo morfológico}

El modelo SAG provee información que nos permite clasificar las galaxias de acuerdo a su morfología y construir la RCM usando exclusivamente galaxias de tipo temprano, 


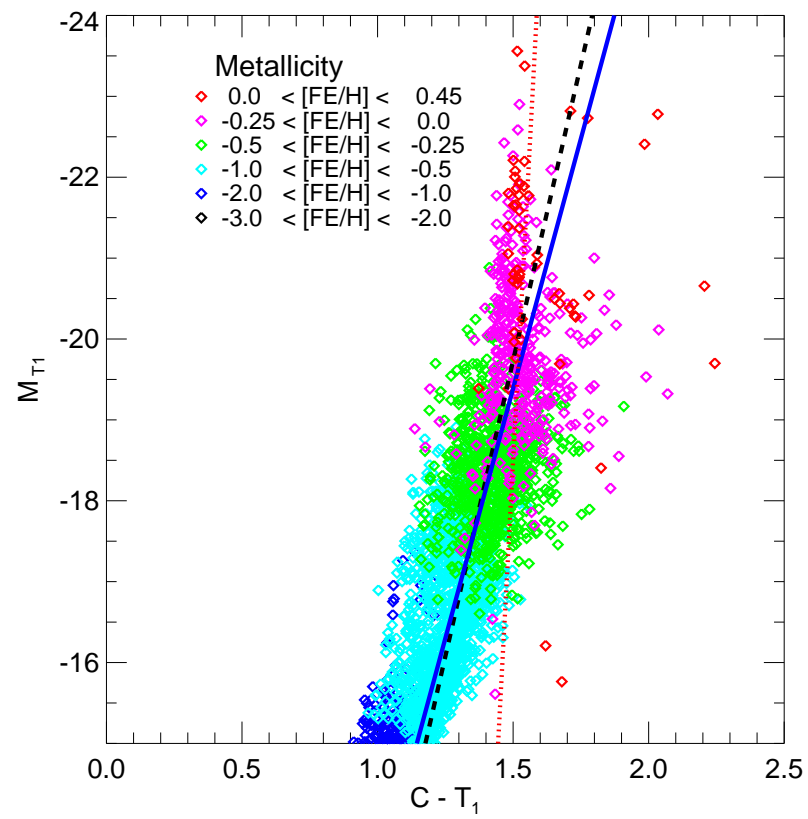

C15

Figura 3.1: RCM obtenida por el modelo semianalítico SAG, aplicado al cúmulo de galaxias simulado C15_3, en el sistema de Washigton (plano $T_{1}$ vs. $\left(C-T_{1}\right)$ ). El criterio de selección de galaxias de la RCM utiliza los colores de las mismas como discriminante (Bell et al., 2004). Los símbolos están coloreados de acuerdo a la abundancia en metales que posee la componente estelar de las galaxias. Se muestran las rectas de ajustes mediante el método de mínimos cuadrados estimadas para el extremo brillante de la RCM (línea puntuada, roja), al extremo débil (línea llena, azul) y a toda la relación (línea de trazos, negra).

en el sentido del criterio de clasificación dado por Hubble Jeans. Como indicador de la morfología se emplea el cociente $B / T$, donde $B$ representa la masa del bulbo de la galaxia y $T$ la masa total de la galaxia. Este cociente está correlacionado con la clasificación de Hubble de tipo T, que consiste en una selección de galaxias basada en la identificación de características tales como brazos espirales, colas de estrellas debidas a las interacciones entre galaxias, barras y bulbos (ver capítulo 1.2). Mientras que los tipos morfológicos se pueden distinguir con alguna certeza en las galaxias cercanas observadas con buena resolución, esto no es posible para las galaxias más débiles y distantes. Por ello, la relación del cociente $B / T$ y la morfología de las galaxias resulta, en mayor o menor medida, subjetiva y exhibe una dispersión considerable (Simien\& deVaucouleurs, 1986; Baugh, 1996). Por otro lado, en la banda $B$, las galaxias con un cociente $B / T<0.4$ corresponden aproximadamente a galaxias de tipo tardío o galaxias espirales, y aquéllas con $B / T>0.6$ coinciden mayormente con galaxias elípticas. Para identificar en el modelo galaxias dominadas por bulbos, fijamos el cociente en $B / T>0.85$. Con esta elección, podemos reproducir las diferentes distribuciones de las fracciones morfológicas en función de la masa estelar de las galaxias (Conselice, 2006).

Con el fin de estudiar las variaciones de la distribución de las galaxias en la RCM causadas por esta selección morfológica, definimos el parámetro $(B / T)_{\mathrm{RCM}}$ por encima del cual los cocientes $B / T$ corresponden a galaxias elípticas que pueblan la RCM. Hacemos variar este parámetro extendiendo el rango de estudio a $0.2<(B / T)_{\mathrm{RCM}}<1.0$. En la figura 3.2, se muestra la RCM del cúmulo C15_3 en el sistema de Washington, donde las galaxias se han seleccionado de acuerdo al criterio morfológico considerando 

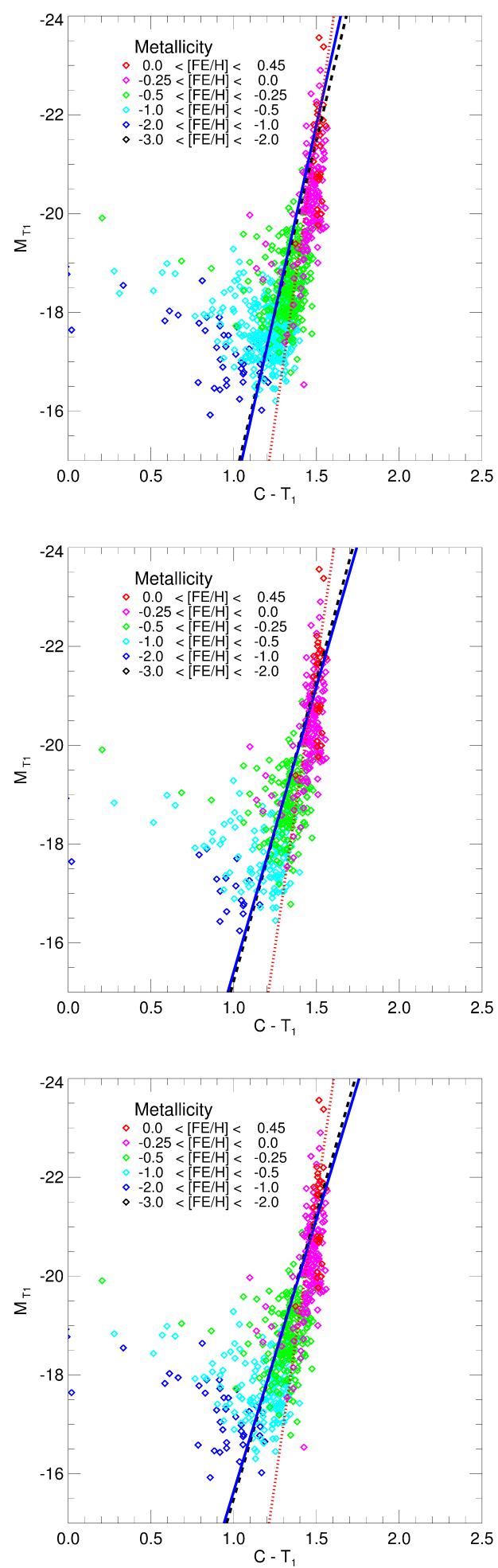

Figura 3.2: RCM del cúmulo C15_3 en el sistema de Washington, donde las galaxias se seleccionan de acuerdo al criterio morfológico de Hubble utilizando distintos valores del parámetro $(B / T)_{\mathrm{RCM}}$ : 0,6 (panel superior), 0,97 (panel medio), y 0,85 (panel inferior). Las rectas de ajustes por mínimos cuadrados a distintas secciones de la relación, así como la codificación de los colores, es igual a la de la figura 3.1. 
tres valores distintos de $(B / T)_{\mathrm{RCM}}$. Los símbolos de colores presentan la misma codificación usada en la figura 3.1 para indicar las metalicidades de las galaxias. En los paneles superior y medio de esta figura se muestran las RCM resultantes de aplicar los criterios $B / T>(B / T)_{\mathrm{RCM}}=0,6$ y $B / T>(B / T)_{\mathrm{RCM}}=0,97$, respectivamente. Estos son valores extremos tales que $(B / T)_{\mathrm{RCM}}=0,6$ incluye tanto galaxias con bulbos pequeños como grandes, mientras que $(B / T)_{\mathrm{RCM}}=0,97$, selecciona casi exclusivamente galaxias dominadas por bulbos. En el panel inferior, la RCM es obtenida considerando $(B / T)_{\mathrm{RCM}}=0,85$ que resulta más adecuado para lograr un mejor acuerdo con datos observados, como se mencionó anteriormente. Como puede verse, en todos los casos, la distribución de galaxias en el extremo débil de la RCM $\left(M_{T 1}>-20\right)$ evidencia un déficit de galaxias, si se compara con la RCM obtenida con el criterio de colores de Bell et al. de la figura 3.1. A la vez, las RCM presentan una dispersión de la distribución de galaxias hacia colores más azules que forman la "nube azul".

Como primera hipótesis, suponemos que las galaxias que se distribuyen hacia el azul en la figura 3.2 son de tipo tardío que han sido mal clasificadas como galaxias de tipo temprano. Sin embargo, al variar los valores del parámetro $(B / T)_{\mathrm{RCM}}$, encontramos que no hay cambios en la dispersión de la distribución de galaxias, ni se obtiene un extremo débil más poblado. De hecho, no reviste de sentido físico considerar como elípticas a galaxias que posean un cociente $B / T$ por debajos de valores límite en el rango $0,2<$ $(B / T)_{\mathrm{RCM}}<0,5$. Hemos considerado estos valores bajos con el sólo propósito de analizar la dependencia de la distribución de galaxias débiles con los valores que toma el parámetro $(B / T)_{\mathrm{RCM}}$. En general, hallamos que las distribuciones de fracciones de diferentes tipos morfológicos en función de la masa estelar de las galaxias se comportan de manera similar para $0,5<(B / T)_{\mathrm{RCM}}<1$, aunque para valores de $(B / T)_{\mathrm{RCM}}$ entre 0,5 y 0,8 se producen fracciones de galaxias espirales menores que las reportadas por Conselice (2006).

Del análisis anterior inferimos que la mayoría de las galaxias que están presentes en el extremo débil de la RCM seleccionada por color (figura 3.1) y que no aparecen en el extremo débil de las RCM seleccionadas por morfología (figura 3.2), son galaxias sin bulbo que evolucionaron pasivamente. Aunque las observaciones indican que las galaxias de tipo tardío pueden contaminar la RCM, la fracción de galaxias encontradas en nuestro modelo es mucho mayor que la reportada en las observaciones. Esto podría ser un defecto del código semianalítico SAG relacionado con el modelado de la inestabilidad del disco galáctico y con la falta de fusiones entre sistemas pequeños, consecuencia de la resolución numérica limitada de la simulación de base de $N$-cuerpos/SPH. Es notable que, aun cuando los criterios morfológicos presentan problemas para poblar el extremo inferior de la RCM, el extremo brillante queda conformado por galaxias de tipo temprano $((B / T>$ $0,85))$ y presenta características similares a las que muestra la RCM seleccionada por el criterio de Bell et al.

\subsubsection{Criterio de selección por formación estelar específica}

La selección de galaxias pertenecientes a la RCM también puede hacerse utilizando una o varias características combinadas, distintivas de las ETGs. En este sentido, cabe destacar el trabajo de Weinmann et al. (2006), quienes estudian la población de grupos de galaxias locales basándose en datos provistos por SDSS, en particular, usando el catálogo New York University Value-Added Galaxy Catalogue (NYU-VAGC) (Blanton et al., 2005). Haciendo uso de la bimodalidad encontrada tanto en la distribución de colores de las galaxias como en la tasas de formación estelar, diferencian dos poblaciones de galaxias, la de tipo tardío y la de tipo temprano, siendo ésta última formada por aquellas galaxias que presentan colores rojos y formación estelar específica (SSFR, por sigla en inglés, Specific Star Formation Rate) baja. Se define la cantidad SSFR como el cociente entre la formación estelar de una galaxia y su masa total. Weinmnann et al. proponen un corte en la distribución de colores para distinguir las galaxias rojas de las azules, dado por la relación 


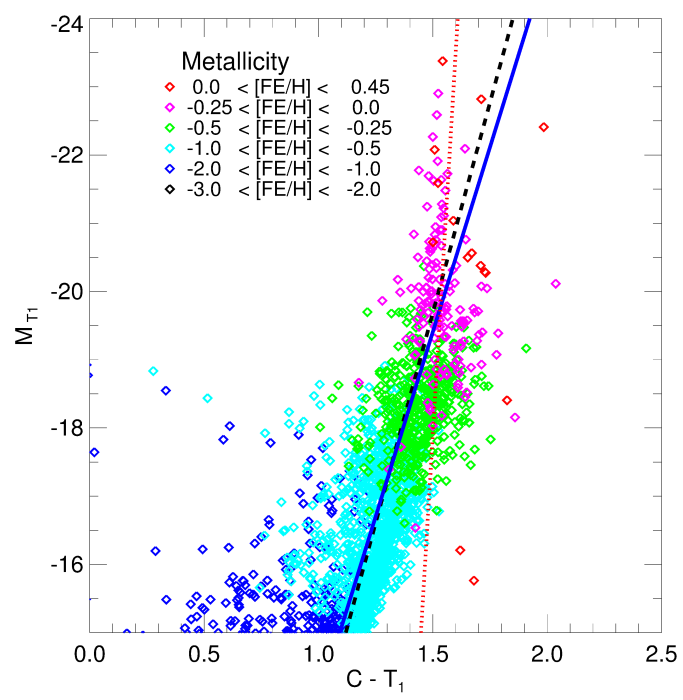

Figura 3.3: RCM del cúmulo C15_3, en el sistema de Washington, obtenida con el criterio de selección de galaxias pasivas dado por Weinmann et al. (2006). Las rectas de ajustes por mínimos cuadrados a distintas secciones de la relación, así como la codificación de los colores, es igual a la de la figura 3.1.

$$
(g-r)=0,7-0,032\left(M_{\mathrm{r}}-5 \operatorname{logh}+16,5\right),
$$

donde $M_{\mathrm{r}}$ es la magnitud absoluta de las galaxias en la banda $r$ de SDSS, corregidas por el efecto $K$, a redshift $z=0,1$. Por otro lado, el corte para diferenciar galaxias activas de pasivas establece que las galaxias serán pasivas si su SSFR es menor que

$$
\log (S S F R)=-10,0+0,094\left(M_{\mathrm{r}}-5 \operatorname{logh}+15,0\right) .
$$

Este criterio nos provee de una forma novedosa de selección de galaxias de la RCM. En la figura 3.3 se muestra la RCM obtenida de nuestro modelo SAG a partir de la implementación de este criterio de selección de galaxias pasivas propuesto por Weinmann et al. (2006). En cuanto a su criterio por color dejamos de lado el análisis, pues ya hemos considerado un criterio similiar en la sección 3.1.1, por lo que sólo presentamos la RCM obtenida de aplicar el criterio que vincula a las galaxias que forman la relación con su actividad de formación estelar. Al comparar esta RCM con la obtenida a partir del criterio de Bell et al., notamos que hay más galaxias brillantes en esta última. Las mismas corresponden a un conjunto de galaxias masivas, rojas y activas que presentan formación estelar residual a bajo redshift.

\subsubsection{Discusión sobre los criterios de selección de galaxias}

Del análisis de la RCM según diferentes criterios de selección de galaxias descriptos en las secciones anteriores, podemos concluir que, independientemente del criterio utilizado, la RCM muestra características comunes en todas los casos. En particular, es posible distinguir un apartamiento de las galaxias más brillantes de la RCM con respecto a la tendencia mostrada por las galaxias más débiles. Este quiebre ocurre en la magnitud 
$M_{T 1}^{\text {quiebre }} \approx-20$. Las galaxias más brillantes que esta magnitud de quiebre presentan un color casi constante $\left(C-T_{1} \approx 1,5\right)$. Tomamos como referencia este quiebre para referirnos a las galaxias "brillantes/débiles" como aquéllas que tienen magnitudes mayores/menores que la magnitud del quiebre. También usaremos la expresión "extremo brillante/extremo débil" para aludir a las respectivas secciones de la RCM. La masa media de las galaxias con la magnitud del quiebre es $\sim 10^{10} M_{\odot}$. El entendimiento de los procesos físicos que dan lugar a esta particular característica de la RCM motiva la investigación que desarrollamos en este trabajo.

Dentro del conjunto de características de la RCM que resultan comunes independientemente del criterio de selección empleado encontramos, además del cambio de pendiente de la RCM alrededor de la magnitud $M_{\mathrm{T} 1} \simeq-20$, que las metalicidades se incrementan con la masa y la luminosidad de las galaxias, y que el extremo brillante está presente, mientras que el extremo débil muestra una dispersión que varía según el criterio de selección adoptado. La intersección de los tres criterios de selección vistos en este capítulo tiene como factor común galaxias rojas, con poca formación estelar y dominada por bulbos que pueblan el extremo brillante de la RCM. Por ello, indistintamente del criterio utilizado, la selección de galaxias engloba las propiedades que distinguen a una población prototípicamente de tipo temprano. Las conclusiones del estudio, por tanto, pueden aplicarse a las ETGs.

Dado nuestro interés en desentrañar las causas físicas que explican el quiebre de la RCM en el extremo brillante, proseguimos el estudio utilizando una selección de galaxias que sea lo menos restrictiva posible. La selección mediante un corte que explota la distribución de color de las galaxias en el diagrama color magnitud parece la más adecuada ya que no introduce ninguna nueva hipótesis sobre los procesos que ocurren en las galaxias, como por ejemplo una tasa de formación estelar baja, o una restricción sobre las morfologías de las mismas. Los colores, además, tienen la ventaja de ser asociados naturalmente con la SED de las galaxias, la extinción por polvo interestelar y la evolución química de las poblaciones estelares que las componen.

Dado que, en las RCM simuladas, el quiebre en el extremo brillante emerge independientemente del criterio usado para seleccionar galaxias, elegimos para este trabajo el criterio de selección propuesto por Bell et al. (2004). Éste, además de resultar poco restrictivo, es de gran utilidad para seleccionar galaxias a más alto redshift de manera simple sin hacer uso de hipótesis ad hoc, permitiendo extender a futuro la investigación desarrollada en este Trabajo de Tesis.

Vale recordar que la denominación "secuencia roja" presente en la literatura responde a una motivación de tipo operacional, surgida a la luz de relevamientos observacionales de alta resolución y de gran cantidad de datos, como el SDSS, junto con el descubrimiento de la bimodalidad en la distribución de los colores en el diagrama color magnitud (Strateva et al., 2001; Blanton et al., 2003; Baldry et al., 2004, 2006), donde resulta más sencillo y natural clasificar los objetos por su color. Los trabajos pioneros en el tema se referían a la ajustada correlación entre los colores y las luminosidades de las galaxias de tipo temprano en el diagrama color magnitud como "relación color magnitud", para distinguirla de la nube de galaxias tardías y azules del diagrama. En este capítulo, demostramos que las galaxias seleccionadas por color definen una RCM que presenta diferencias leves y localizadas, con respecto a la RCM seleccionada por otros criterios. Las diferencias se hallan principalmente, en el extremo débil de la relación. Por ello, de manera más abarcativa, en este trabajo nos referimos a las galaxias que forman la "relación color magnitud", aun cuando las mismas han sido seleccionadas mediante un criterio de corte por color, y tal vez resultase, por ello, más natural referirse a la relación que forman como "secuencia roja". 


\subsection{Transformaciones entre sistemas fotométricos}

Para cuantificar la presencia del quiebre en la RCM, nuestro estudio se centra en el análisis de los cambios de pendiente entre el extremo brillante con respecto a la pendiente de la RCM completa. Por ello, dejamos de lado el ajuste de los puntos de cero y, por ende, no necesitamos convertir los datos observacionales de magnitudes absolutas en magnitudes aparentes y viceversa. Si bien las magnitudes obtenidas de SAG tienen en cuenta el efecto de extinción por polvo interestelar (ver el capítulo 2), las mismas no están influenciadas por la extinción debida a la presencia de polvo intergaláctico.

Las magnitudes de las galaxias del modelo SAG están dadas en el sistema JohnsonMorgan. Para comparar la RCM simulada con relaciones dadas por datos observacionales necesitamos transformar las magnitudes del sistema Johnson-Morgan a los diferentes sistemas fotométricos utilizados en la literatura.

Cada una de las bandas fotométricas elegidas para el estudio ofrece ventajas para medir con mayor precisión diferentes características de las galaxias observadas. Destacamos cuatro planos color magnitud que permiten obtener información de la comparación de las RCM en cada uno, y que a la vez, proveen un amplio set de testeos del modelo. Presentamos a continuación las bandas utilizadas.

- $(U, R)$ : Esta banda es sensible a la edad de las galaxias. Un ejemplo típico de la bimodalidad en la distribución de colores en el diagrama color magnitud, y de la presencia del quiebre puede verse en la figura 1.3, perteneciente al trabajo de Baldry et al. (2006).

- $(V-I)$ : Esta es un banda clásica en la que abundan datos observacionales de cúmulos de galaxias del universo local (Misgeld et al., 2008; Mieske et al., 2007; Misgeld et al., 2009; de Rijcke et al., 2009)

- $(B-R)$ : En esta banda, López-Cruz et al. (2004) presenta una muestra amplia de la RCM de 57 cúmulos de galaxias a bajo redshift, con diferentes densidades, tipos de cúmulos y luminosidades en rayos $\mathrm{X}$.

- $\left(C-T_{1}\right)$ : Sistema fotométrico de Washington, creado por Canterna\& Harris (1979). Los colores integrados $\left(C-T_{1}\right)$ han probado ser de gran sensibilidad a la metalicidad de las poblaciones estelares envejecidas, como cúmulos globulares (ver Harris \& Harris 2002; Forte et al. 2007). Cabe destacar el trabajo de Smith Castelli et al. (2008), quienes estudian en este sistema fotométrico la RCM del cúmulo de Antlia y otras relaciones de escala.

Las magnitudes $R$ e $I$ consideradas en los trabajos observacionales que discutiremos más adelante están dadas en el sistema fotométrico de Cousins. Las magnitudes $R_{\mathrm{J}}$ e $I_{\mathrm{J}}$ dadas por el modelo SAG en el sistema fotométrico de Johnson-Morgan son transformadas a $R_{\mathrm{C}}$ e $I_{\mathrm{C}}$ en el sistema de Cousins, usando relaciones para ETGs dadas por Fukugita et al. (1995). De su tabla 3b extraemos las diferencias entre las magnitudes para los tipos morfológicos E y S0,

$$
\begin{gathered}
R_{\mathrm{J}}-R_{\mathrm{C}}=\frac{-0,11-0,08}{2}=-0,095, \\
I_{\mathrm{J}}-I_{\mathrm{C}}=\frac{-0,14-0,11}{2}=0,125 .
\end{gathered}
$$

Convertimos la magnitud $V$ del sistema de Johnson-Morgan y la magnitud $I_{\mathrm{C}}$ al sistema de Washington $\left(C-T_{1}\right)$, a través de la transformación 

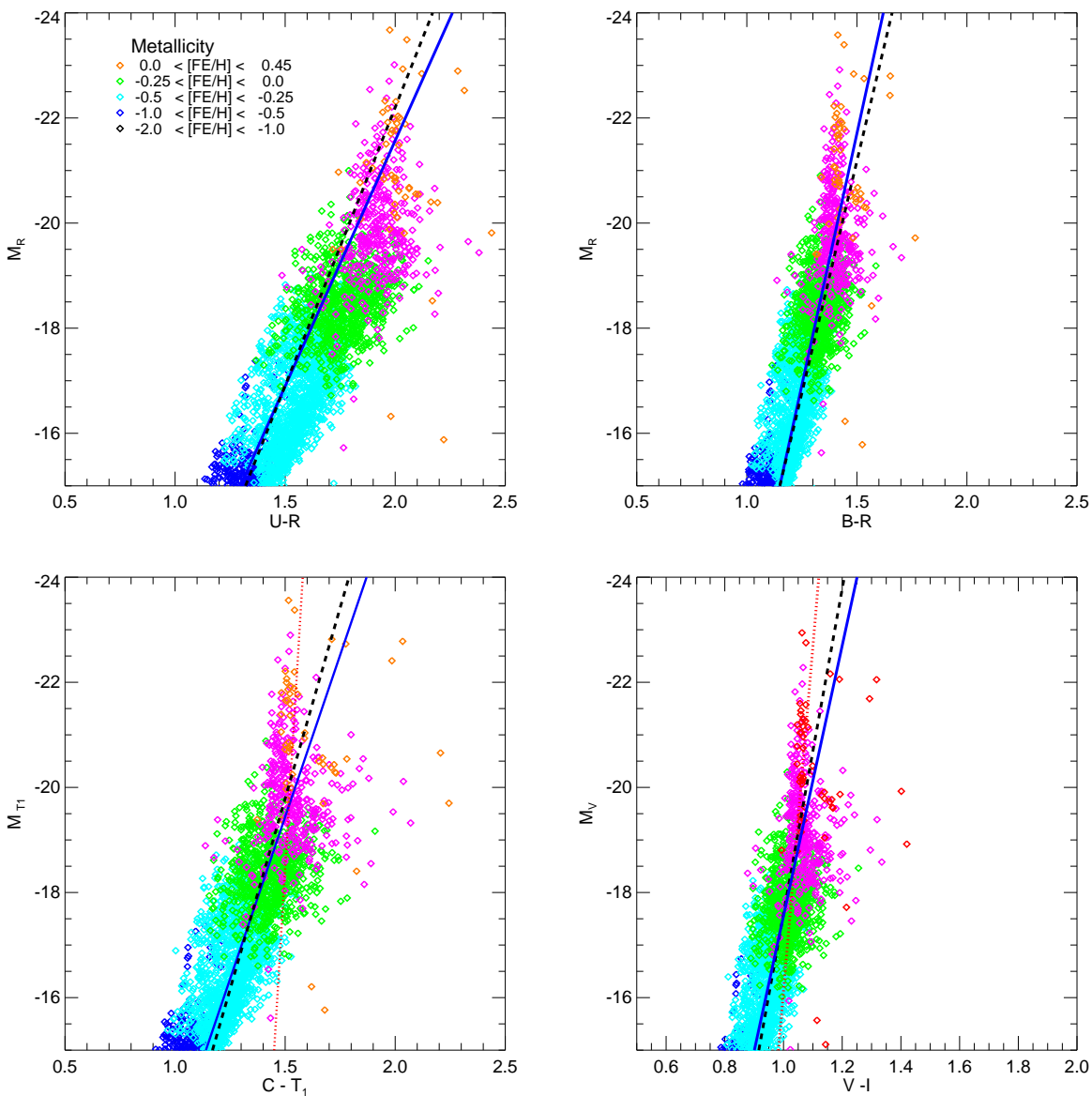

Figura 3.4: Relaciones color magnitud obtenidas por el modelo semianalítico SAG aplicado al cúmulo de referencia C15_3 (ver la tabla 3.1) en cuatro planos color magnitud: $R$ vs. $(U-R)$ (panel superior izquierdo), $R$ vs. $(B-R)$ (panel superior derecho), $T_{1}$ vs. $\left(C-T_{1}\right)$ (panel inferior izquierdo), y $I$ vs. $(V-I)$ (panel inferior derecho). Los símbolos se colorean de acuerdo a las abundancias químicas de las galaxias. Se muestran las rectas de ajustes por mínimos cuadrados al extremo débil de la RCM (línea llena azul), al extremo brillante (línea roja punteada), y a toda la relación (línea negra de trazos).

$$
\left(V-I_{\mathrm{C}}\right)=0,49\left(C-T_{1}\right)+0,32
$$

dada por Forbes \& Forte (2001) para cúmulos globulares. Finalmente, obtenemos la magnitud $T_{1}$ transformando la magnitud $R$ de Johnson-Morgan a $R_{\mathrm{C}}$ de Cousins, mediante la ecuación 3.4. Luego pasamos al sistema de Washington por la relación

$$
R_{\mathrm{C}}-T_{1} \sim-0,02
$$

dada por Geisler (1996).

En la figura 3.4 mostramos la RCM del cúmulo de galaxias de refencia (C15_3) en los diferentes planos color magnitud, esto es, $R$ vs. $(U-R), R$ vs. $(B-R), I$ vs. $(V-I), \mathrm{y}$ $T_{1}$ vs. $\left(C-T_{1}\right)$. Se muestra en la figura 3.5 la RCM de uno de los cúmulos menos masivos (C14_1) en los mismos planos color magnitud. 

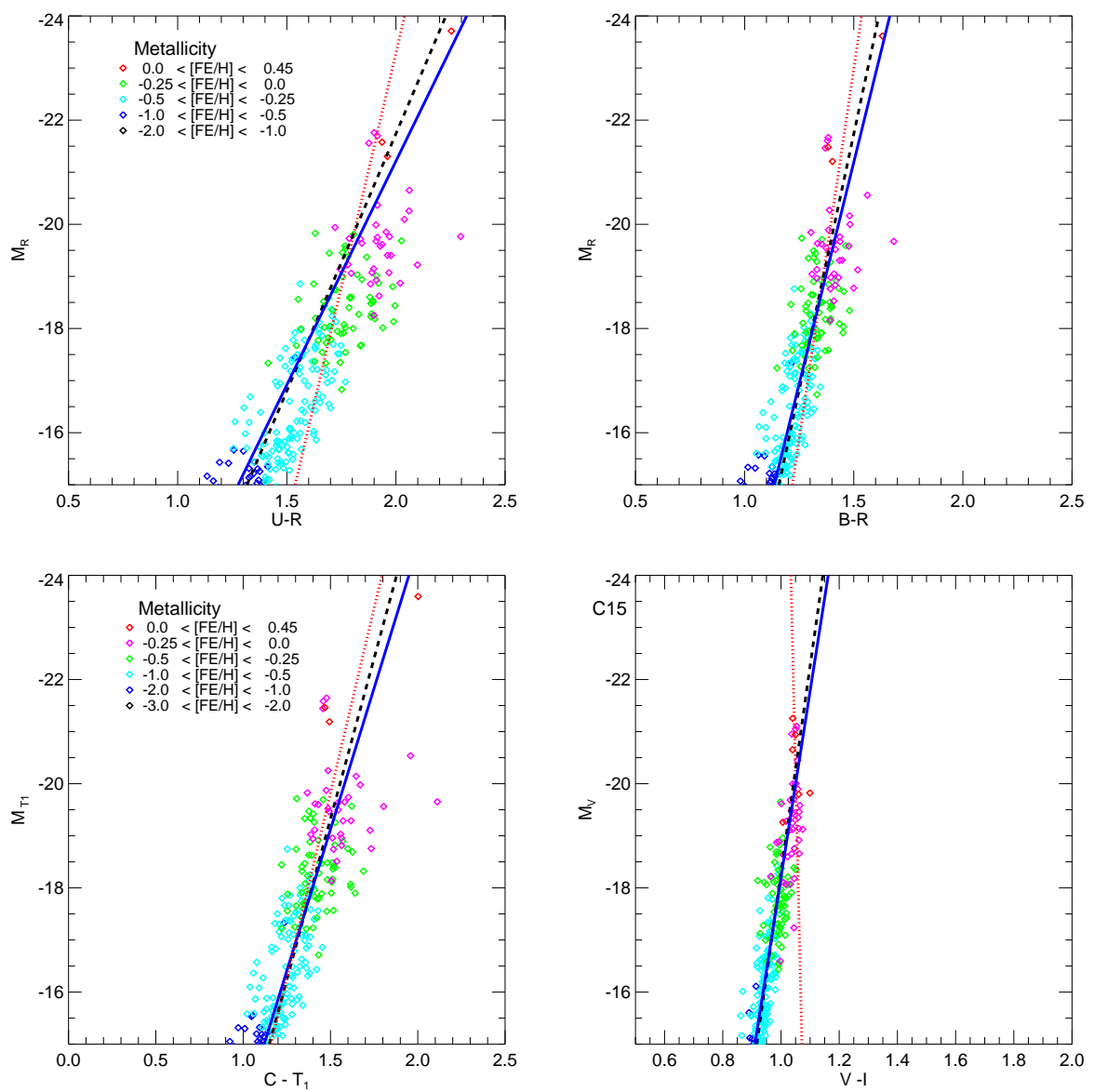

Figura 3.5: Relaciones color magnitud obtenidas por el modelo semianalítico SAG aplicado al cúmulo C14_1 (ver la tabla 3.1) en cuatro planos color magnitud: $R$ vs. $(U-R)$ (panel superior izquierdo), $R$ vs. $(B-R)$ (panel superior derecho), $T_{1}$ vs. $\left(C-T_{1}\right)$ (panel inferior izquierdo), y $I$ vs. $(V-I)$ (panel inferior derecho). Los símbolos se colorean de acuerdo a las abundancias químicas de las galaxias. Se muestran las rectas de ajustes por mínimos cuadrados al extremo débil de la RCM (línea llena azul), al extremo brillante (línea roja punteada), y a toda la relación (línea negra de trazos). 


\subsection{La pendiente de la RCM en diferentes bandas fotométricas}

Una vez que estamos en condiciones de pasar del sistema fotométrico usado en SAG a otras bandas, obtenemos la RCM del cúmulo de referencia, $\mathrm{C} 15_{3}$, en los cuatro planos de color magnitud detallados en la sección anterior; las RCM se muestran en en la figura 3.4. En todos los planos es posible distinguir el apartamiento de las galaxias brillantes de la RCM con respecto a la tendencia mostrada por las galaxias más débiles, tal como se resaltó al comparar las RCM obtenidas mediante distintos criterios de selección (ver discusión en sección 3.1.4). Este quiebre ocurre aproximadamente a la misma magnitud en todas las bandas fotométricas $\left(M_{R}^{\text {quiebre }} \sim M_{V}^{\text {quiebre }} \sim M_{T 1}^{\text {quiebre }} \approx-20\right)$, siendo más evidente en el sistema de Washington.

A fin de describir cuantitativamente la forma de la RCM, calculamos ajustes a la distribución de galaxias de la RCM en cada una de las bandas fotométricas consideradas. El método utilizado es la técnica de mínimos cuadrados que minimiza la distancia entre la posición de las galaxias del modelo en el plano color magnitud y la recta de mejor ajuste; las diferencias son tomadas en sentido vertical, correspondientes a las magnitudes de las galaxias. Los ajustes son calculados con la rutina "Nonlinear least-squares" del Lenguaje de Programación IDL (David Stern \& (ITT VIS), 1977). Nuestro objetivo es testear el modelo SAG mediante la comparación de las pendientes de las RCM en las diferentes bandas fotométricas con aquéllas disponibles en estudios observacionales. No buscamos establecer una predicción del valor último de la pendiente de la RCM.

De este modo, considerando la RCM en distintas bandas de cada uno de los ocho cúmulos de galaxias descriptos en la sección 3 , se realizan los ajustes mediante mínimos cuadrados al extremo débil de la RCM, al extremo brillante y a toda la relación, para lo cual consideramos los rangos de magnitudes [-16, -20], [-20,-24], y [-15, -24], respectivamente; estos rangos son válidos para cualquiera de las bandas fotométricas consideradas. Los valores de las pendientes de los ajustes realizados a toda la relación y al extremo débil son volcados en la tabla 3.1. Los valores de las pendientes de las rectas de ajuste al extremo brillante de la RCM no aparecen en esta tabla pues intervienen en el análisis desarrollado en el capítulo 6. En las figuras 3.1, 3.2 y 3.3, que muestran las $\mathrm{RCM}$ para el cúmulo $\mathrm{C} 15_{3}$ obtenidas mediante diferentes criterios de selección de galaxias (sección 3.1), se grafican las correspondientes rectas de ajuste; allí la línea negra de trazos representa el ajuste a toda la relación, mientras que las líneas sólida azul y roja punteada corresponden al extremo débil y brillante de la RCM, respectivamente.

Notése que las pendientes en la tabla 3.1, designadas como $b$, corresponden a relaciones color magnitud donde la variable independiente está dada por el color de las galaxias. Para poder comparar las pendientes obtenidas con resultados observacionales debemos invertir las pendientes observadas $(1 / b)$ en los casos en los que la RCM se ha calculado tomando al color como variable dependiente. Esto es equivalente a rotar en $90^{\circ}$ los diagramas color magnitud de, por ejemplo, la figura 3.1.

Los valores de las pendientes de la RCM que resultan de nuestros cálculos son comparados con valores obtenidos de estudios observacionales existentes en la literatura. Consideramos el trabajo de López-Cruz et al. (2004), quienes presentan una muestra amplia de la RCM de 57 cúmulos de galaxias a bajo redshift, con diferentes densidades, tipos de cúmulos y luminosidades en rayos X. Los ajustes a las RCM se calculan en el plano $R$ vs. $(B-R)$, como muestran en la tabla 1 de su trabajo. Extrayendo los datos de dicha tabla, calculamos un promedio simple para hallar el valor medio de la pendiente de los 57 cúmulos. Éste resulta de $1 / b_{\mathrm{BR}}=-0,051 \pm 0,002$, o bien, $b_{\mathrm{BR}}=-19,61$ para comparar con nuestras simulaciones. Este valor pertenece al rango de valores correspondiente al ajuste de "Toda la RCM", presentado en nuestra tabla 3.1.

En el trabajo de Misgeld et al. (2008), se ajusta la RCM de una muestra de galaxias enanas de tipo temprano $\left(M_{\mathrm{V}}>-17\right)$ del cúmulo de Hydra I en el plano $(V-I)$ vs. $V$, obteniendo una pendiente $1 / b_{\mathrm{VI}}=-0,039$ ( $\mathrm{rms}$ del ajuste igual a 0,12$)$, o $b_{\mathrm{VI}}=-25,64$. Así mismo, en el mismo plano color magnitud, los autores estiman la pendiente de la 
Cuadro 3.1: Pendientes de las rectas de ajuste al extremo débil y a toda la RCM, calculadas mediante el método de mínimos cuadrados para los cúmulos C15 y C14, en diferentes bandas fotométricas.

\begin{tabular}{l|cc|cc|ccc|c} 
& \multicolumn{2}{|c|}{$R$ vs. $B-R$} & \multicolumn{2}{c|}{$V$ vs. $V-I$} & \multicolumn{2}{c}{$T_{1}$ vs. $C-T_{1}$} & \multicolumn{2}{c}{$R$ vs. $U-R$} \\
\hline Cúmulo & Ext. débil & Toda la RCM & Ext. débil & Toda la RCM & Ext. débil & Toda la RCM & Ext. débil & Toda la RCM \\
\hline C14_1 (g1542) & -16.92 & -19.90 & -20.12 & -25.22 & -10.79 & -12.29 & -8.55 \\
C14_2 (g3344) & -18.55 & -20.00 & -19.97 & -27.85 & -12.24 & -13.77 & -12.24 \\
C14_3 (g6212) & -19.23 & -20.88 & -24.65 & -30.37 & -12.36 & -13.80 & -9.58 \\
C14_4 (g676) & -17.43 & -20.38 & -26.47 & -28.95 & -11.45 & -13.83 & -8.75 \\
C14_5 (g914) & -16.39 & -18.99 & -21.48 & -25.31 & -10.47 & -12.35 & -8.30 \\
\hline C15_1 (g1) & -18.10 & -21.36 & -24.78 & -29.72 & -11.48 & -13.96 & -9.00 \\
C15_2 (g8) & -18.11 & -20.69 & -24.12 & -28.84 & -11.55 & -13.71 & -9.79 \\
C15_3 (g51) & -18.92 & -21.96 & -25.71 & -30.91 & -12.35 & -14.63 & -9.25 \\
\hline
\end{tabular}




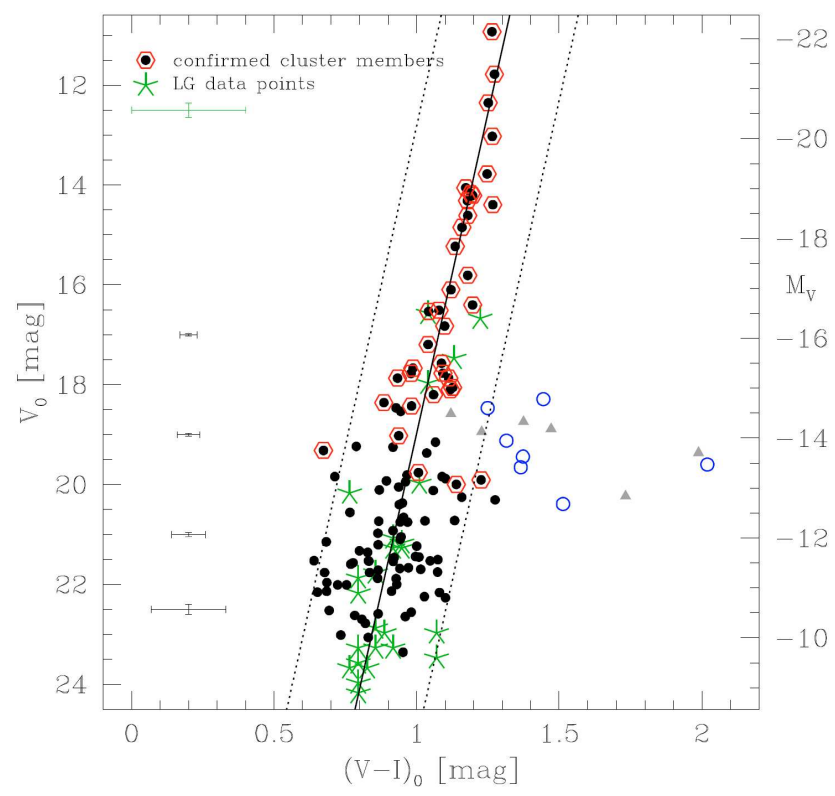

Figura 3.6: Relación color magnitud de las galaxias de tipo temprano del cúmulo de Hydra I, tomada del trabajo de Misgeld et al. (2008) (ver su figura 10). El quiebre en el extremo brillante se ve claramente, aunque los autores no hacen mención al mismo y ajustan una recta a toda la relación.

RCM del Grupo Local de galaxias elípticas enanas (dE, según siglas en inglés, dwarf ellipticals) y de las enanas esferoidales (dSph, según siglas en inglés, dwarf spheroidals). Los valores para las pendientes son $1 / b_{\mathrm{VI}}=-0,038$ ( $\mathrm{rms}$ del ajuste igual a 0,09 ), o $b_{\mathrm{VI}}=-26,32$.

Mieske et al. (2007), por su parte, calculan en el mismo plano la pendiente de la RCM para una muestra de galaxias $\mathrm{dE}\left(M_{\mathrm{V}}>-17\right)$ en el cúmulo de Fornax, obteniendo los valores $1 / b_{\mathrm{VI}}=-0,033 \pm 0,004$, o $b_{\mathrm{VI}}=-30,30$.

El análisis de Misgeld et al. (2009) de la RCM mostrada por ETGs enanas en el cúmulo de Centaurus les permite obtener una pendiente $1 / b_{\mathrm{VI}}=-0,042$ ( $\mathrm{rms}$ del ajuste igual a 0,10$)$, o $b_{\mathrm{VI}}=-23,81$. Excepto la pequeña diferencia del valor mostrado por Mieske et al., todas las pendientes se hallan dentro del rango cubierto por las pendientes del ajuste al extremo débil de las RCM, listados en la tabla 3.1.

El cúmulo de Perseo es estudiado por de Rijcke et al. (2009) con datos de HST/ACS. Estos autores pesentan una RCM global que incluye varios grupos y cúmulos, obteniendo un ajuste común con pendiente $1 / b_{\mathrm{VI}}=-0,033 \pm 0,004$, o $b_{\mathrm{VI}}=-30,30$, que también está de acuerdo con los valores de "Toda la RCM" de la tabla 3.1. Finalmente, Smith Castelli et al. (2008) obtienen la RCM de una muestra de galaxias de tipo temprano ubicadas en la región central de Antlia, en el sistema fotométrico de Washington. Los autores realizan un ajuste de la RCM por el método de mínimos cuadrados que otorga a la relación la siguiente forma funcional

$$
T_{1}=(38,4 \pm 1,8)-(13,6 \pm 1,0)\left(C-T_{1}\right) .
$$

Este ajuste comprende a todas las galaxias de la RCM observada. Las galaxias de la muestra estudiada por Smith Castelli et al. poseen magnitudes más débiles en relación a 
otros cúmulos de galaxias. Debido a esto, no es posible distinguir el quiebre de la RCM para el cúmulo de Antlia.

El ajuste lineal al extremo brillante de la RCM del cúmulo $\mathrm{C} 15_{3}$, en el sistema de Washington, mostrado en la Figura 3.1 en línea roja punteda, tiene la siguiente expresión analítica

$$
M_{T_{1}}=83,65-67,92\left(C-T_{1}\right)
$$

Claramente, la pendiente de este último ajuste difiere de la pendiente del ajuste al extremo débil, que puede expresarse mediante la relación

$$
M_{T_{1}}=0,82-12,38\left(C-T_{1}\right) .
$$

Este ajuste se asemeja al realizado para toda la RCM, dado por

$$
M_{T_{1}}=2,19-14,62\left(C-T_{1}\right) .
$$

La pendiente obtenida por mínimos cuadrados para la RCM del cúmulo C14_3 es -13,77 (como puede verse en la tabla 3.1), en muy buen acuerdo con el valor observado de la pendiente de Antlia dada en la ecuación 3.8. Hallamos resultados similares para todos los cúmulos simulados, como puede verse en la tabla 3.1. Inferimos, pues, que la universalidad de la RCM sugerida por diferentes observaciones de un gran rango de ambientes de cúmulos, es una hipótesis viable (v.g López-Cruz et al., 2004; de Rijcke et al., 2009).

El apartamiento del extremo brillante de la $\mathrm{RCM}\left(-24 \lesssim M_{T_{1}} \lesssim-20\right)$ con respecto al ajuste lineal al extremo débil es claro en los tres cúmulos masivos simulados (C15) (ver figura 3.1, que muestra la RCM del cúmulo de referencia C153), aunque el cambio de pendiente en la RCM de los cúmulos menos masivos (C14) es menos evidente. Esto es principalmente debido a la falta de objetos brillantes en rangos de alta luminosidad en los cúmulos C14, y a la dispersión intrínseca de la RCM que dificulta la visualización de una magnitud de quiebre definida. De todos los cúmulos C14 simulados, sólo dos (C14_1, C14_3) presentan una relación más definida alrededor de la magnitud $M_{T_{1}} \leq-20$, donde el cambio de pendiente se vuelve perceptible; como ejemplo, mostramos en la figura 3.5 los planos color magnitud del cúmulo C14_1. Como mencionamos anteriormente, el quiebre en el extremo brillante está presente en algunas de las RCM observadas. Por ejemplo, la RCM del cúmulo de Hydra I mostrada por Misgeld et al. (2008) (ver figura 3.6) evidencia el quiebre, aunque los autores no hacen mención de ello. Los estudios del cúmulo de Virgo llevados a cabo por Janz \& Lisker (2009) y la muestra de ETGs de Skelton et al. (2009), ambos basados en datos de SDSS, claramente muestran un quiebre en el extremo brillante hacia colores más azules que los que resultarían de extrapolar la tendencia seguida por las galaxias del extremo débil.

Del análisis descripto en esta sección, se desprende que existe un buen acuerdo entre las pendientes de las RCM simuladas y las observadas, especialmente para las galaxias con magnitudes menores (más débiles) que la del quiebre $M_{T 1}^{\text {quiebre }} \sim-20$. Es notable que este acuerdo, así como el quiebre del extremo brillante de la RCM, surgen naturalmente luego de calibrar el código SAG para satisfacer varios observables simultáneamente, como se explicita en el capítulo 2. 

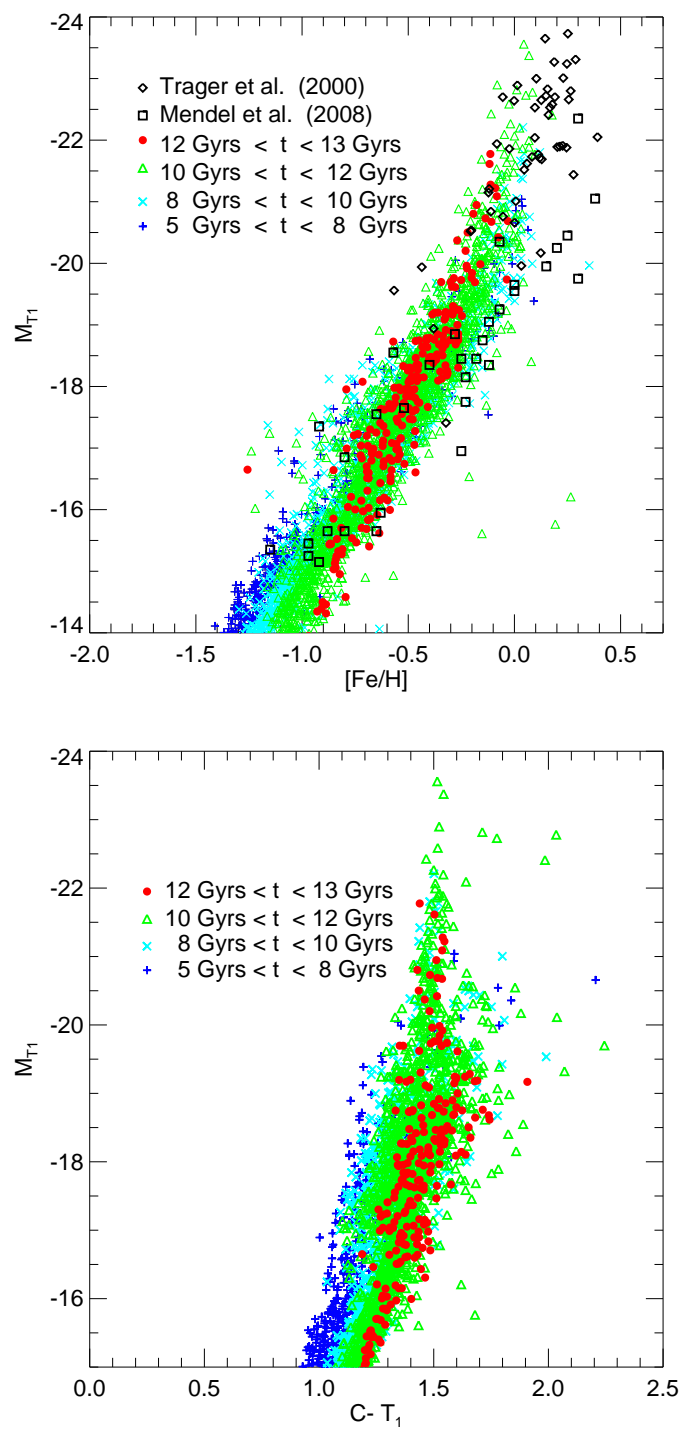

Figura 3.7: Panel superior: Relación luminosidad metalicidad para las mismas galaxias simuladas pertenecientes a la RCM del cúmulo C15_3, mostrada en la figura 3.1. Los símbolos de colores identifican diferentes rangos de edades de las galaxias; los diamantes negros representan las abundancias $[\mathrm{Fe} / \mathrm{H}]$ de la muestra de ETGs presentada por Trager et al. (2000), mientras que los cuadrados negros corresponden a ETGs del grupo NGC 5044 (Mendel et al., 2009). Panel inferior: RCM de las galaxias simuladas en el plano $T_{1}$ vs. $\left(C-T_{1}\right)$, con símbolos coloreados identificando el rango de edades de las galaxias. 


\subsection{Metalicidad de las galaxias de la RCM}

La metalicidad de las galaxias es un marcador sensible a los procesos físicos involucrados en el desarrollo de la RCM. La metalicidad de cada galaxia simulada está dada por la abundancia de hierro de su componente estelar definida mediante el índice $[\mathrm{Fe} / \mathrm{H}]$, considerando el valor solar de la abundancia de hierro por número $(\mathrm{Fe} / \mathrm{H})_{\odot}=2,82 \times 10^{-5}$ (Asplund et al., 2005); la masa de Fe y H contenida en la componente estelar de las galaxias están directamente dadas por el código SAG. En la figura 3.1, así como en el resto de las figuras de este capítulo que muestran la RCM de los cúmulos simulados, los galaxias son identificadas con distintos colores de acuerdo al rango de metalicidad en el cual se encuentran. Podemos ver que las galaxias en el extremo brillante son las más metálicas, con abundancias de $[\mathrm{Fe} / \mathrm{H}] \gtrsim-0,25$, algunas incluso alcanzan valores altos como $[\mathrm{Fe} / \mathrm{H}] \approx 0,43$. El valor medio de su distribución de metalicidades es $\langle[\mathrm{Fe} / \mathrm{H}]\rangle=-0,03 \pm 0,05$. A medida que nos movemos hacia magnitudes más débiles en la RCM, las galaxias se vuelven menos luminosas, más azules y sus valores de metalicidad descienden. En la figura se ve claramente que la RCM puede interpretarse como una relación entre la masa y la metalicidad de las galaxias, como ya se discutió en la sección 3.1.1.

Teniendo en cuenta la presencia de una relación entre la masa y la metalicidad de las galaxias, evaluamos si existe una relación entre la luminosidad y la metalicidad. En el panel superior de la figura 3.7 se muestra la relación luminosidad metalicidad (RLM) para las mismas galaxias que forman la RCM del cúmulo de referencia, $\mathrm{C} 15_{3}$, mostrada en la figura 3.1. Para poder comparar nuestros resultados con datos observacionales, incluimos las abundancias de hierro $[\mathrm{Fe} / \mathrm{H}]$ de una muestra de ETGs pertenecientes al grupo NGC 5044, extraidas del trabajo de (Mendel et al., 2009), y de una muestra descripta por Trager et al. (2000). Esta última contiene miembros de cúmulos (seis del cúmulo de Virgo, once de Fornax, y uno de Abell 194, uno de los cúmulos más ricos), pero la mayoría de las galaxias se encuentra en pequeños grupos de variada riqueza. La metalicidad de este conjunto de galaxias es redeterminado por Trager et al. (2008); las abundancias $[\mathrm{Fe} / \mathrm{H}]$ son obtenidas a partir de estos valores de metalicidad usando una transformación dada por Trager et al. (2000) (ver su ecuación 1). En todos los casos hemos transformado las magnitudes absolutas a la banda $T_{1}$ a través de las relaciones de Fukugita et al. (1995). Como puede verse, los valores observados, los cuales cubren un amplio rango de magnitudes y abundancias, se superponen a la relación simulada. El acuerdo es particularmente bueno para galaxias en el extremo débil de la RCM $\left(M_{T_{1}} \gtrsim-20\right)$, aunque notamos que las abundancias observadas de algunas galaxias en el extremo brillante son levemente más grandes que aquéllas obtenidas de nuestro modelo. Esta discrepancia puede ser debida al hecho de que la mayoría de las galaxias en la muestra observada pertenecen a entornos de relativamente baja densidad, y tienen diferentes historias de formación estelar y enriquecimiento químico que aquéllas que habitan en cúmulos masivos. El hecho de que las metalicidades de las galaxias a $z=0$ satisfagan restricciones impuestas por las observaciones es particularmente relevante para nuestro estudio. El mismo avala el uso de las historias químicas de las galaxias como una herramienta que ayude a entender el desarrollo de la RCM y su especial característica en el extremo brillante.

Las galaxias en la RLM de la figura 3.7 (panel superior) han sido identificadas con diferentes colores de acuerdo al rango de edad al cual pertenecen. Las edades han sido estimadas considerando el promedio pesado con la masa estelar del tiempo de nacimiento de cada población estelar individual. Para las galaxias menos luminosas $\left(M_{T_{1}} \gtrsim-16\right)$, resulta evidente la clara correlación existente entre edad y metalicidad. Las galaxias más jóvenes son más pobres en metales, por lo que se encuentran en el lado izquierdo de la RLM, mientras que las galaxias más viejas tienen abundancias de hierro más altas y se localizan hacia la derecha de esta relación. A medida que nos movemos a lo largo de la RLM hacia galaxias más brillantes, las edades y las metalicidad pasan a estar anticorrelacionadas. Este hecho es consistente con la tendencia encontrada por Gallazzi et al. (2006) para galaxias del SDSS para una dispersión de velocidades fija. Esta anticorre- 
lación podría explicar la modesta dispersión del extremo brillante de la RCM (Trager et al., 2000), que se caracteriza por tener galaxias dentro de un rango de edades muy acotado, consistente también con resultados observacionales de Gallazzi et al. (2006). La mayoría de las galaxias del extremo brillante de la RCM simulada presentan edades similares $\left(1,0 \times 10^{10} \mathrm{yr}<t<1,2 \times 10^{10} \mathrm{yr}\right)$. Esto puede verse en el panel inferior de la figura 3.7, que muestra la RCM en el plano $\left(C-T_{1}\right)$ versus $T_{1}$, donde las galaxias están identificadas con símbolos de diferentes colores de acuerdo a su edad. De esta figura, resulta claro que en el extremo débil, la dispersión en color alrededor de la RCM para una luminosidad fija se debe enteramente a las variaciones de edad de las galaxias, tal como supone Bernardi et al. (2005), donde las galaxias más jóvenes correlacionan con colores más azules. A medida que nos desplazamos hacia luminosidades mayores, las galaxias de una cierta edad se vuelven más rojas debido a que son más ricas en metales. Las diferencias de edad de las galaxias del extremo brillante de la RCM no producen un efecto significativo en los colores finales de las galaxias (ver figura 2.4), por lo que el comportamiento de las galaxias del extremo brillante de la RCM se puede atribuir a los efectos de la metalicidad. En los capítulos siguientes, analizamos las razones físicas que dan lugar a que las galaxias viejas que se ubican en el extremo brillante de la RCM alcancen un valor máximo de metalicidad. Nuestro objetivo es demostrar que este efecto de la metalicidad es responsable de los colores alcanzados por las galaxias masivas, que son más azules que los que se obtendrían a partir de una extrapolación del extremo débil de la RCM. 
$\Gamma_{\text {Capítulo }}$

\section{Procesos físicos involucrados en el desarrollo de la RCM}

Las propiedades de las galaxias resultan de la compleja combinación de todos los procesos físicos incluídos en el código semianalítico (ver capítulo 3) que permiten modelar la formación y evolución de las galaxias. Todos estos procesos influyen directa o indirectamente en la determinación de la evolución de la tasa de formación estelar de las galaxias que finalmente define las masas, los colores y las metalicidades de las mismas. A su vez, estas propiedades determinan la RCM de las galaxias. A fin de identificar qué mecanismos son responsables de las diferentes características mostradas por la RCM y, en particular del quiebre en el extremo brillante, en este capítulo presentamos el estudio de la evolución de las masas y metalicidades de la componente estelar de cada galaxia como resultado de diferentes tipos de eventos que conducen a la formación y acreción de estrellas.

\subsection{Formación y acreción de la masa estelar}

En el modelo SAG, las estrellas se originan mediante:

- Formación estelar en modo pasivo, que consume en forma gradual el gas frío disponible en los discos galácticos de acuerdo a la formulación dada en el capítulo 2 .

- Brotes de formación estelar desatados por la compresión del gas frío de las galaxias, debida a diferentes tipos de interacciones sufridas por las mismas:

- Interacciones que dan lugar a la formación estelar en galaxias cuyo disco es dinámicamente inestable (criterio dado por la ecuación 2.8).

- Fusiones entre galaxias. Las fusiones pueden ser mayores o menores dependiendo del cociente entre las masas bariónicas de las galaxias involucradas. En el caso de una fusión mayor, todo el gas frío disponible en el remanente es consumido en un brote de formación estelar. En el caso de una fusión menor, la presencia de un brote estelar dependerá del gas frío disponible en el remanente y de la relación de masas entre las galaxias central y satélite; estas cantidades deben ser superiores a un dado valor umbral, tal como se describe en detalle en el capítulo 3 . 
A su vez, la masa estelar en las galaxias puede aumentar debido a la acreción de estrellas provenientes de las galaxias satélites que se fusionan con las primeras. En lo que sigue, la masa estelar generada durante cualquier tipo de evento de formación estelar será denotada con el término "stars", mientras que la masa estelar agregada durante las fusiones galácticas (que ya se encontraba presente como estrellas en la galaxia satélite), será referida como "sat". Por lo tanto, las etiquetas "stars" y "sat" indican la clase de componente estelar que se incorpora por cada proceso. En consecuencia, la masa estelar de una dada galaxia $g$, a un cierto redshift $z$, que resulta de la incorporación de una dada componente ("comp"), de un dado proceso ("proc"), se indicará como $S M_{g, z}^{\text {proc, comp }}$.

Teniendo en cuenta las definiciones anteriores, implementamos en el modelo semianalítico SAG un conjunto de variables que tienen en cuenta todas las contribuciones a la masa estelar de una dada galaxia debidas a los procesos físicos involucrados. Cabe destacar que estas variables no afectan el funcionamiento del código sino que fueron introducidas con el objetivo de realizar un análisis detallado de los resultados del modelo, especialmente referente al desarrollo de la RCM. Específicamente consideramos:

(I) Masa estelar proveniente de formación estelar pasiva (quiescent):

- $S M_{g, z}^{\text {quiescent, stars }}$

(II) Masa estelar proveniente de formación estelar en eventos de inestabilidades de disco:

- $S M_{g, z}^{\text {disc, stars }}$

(III) Masa estelar proveniente de fusiones galácticas de diferentes tipos:

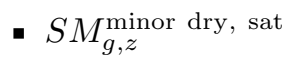

- $S M_{g, z}^{\operatorname{minor} \text { wet, sat }}$

- $S M_{g, z}^{\operatorname{minor} \text { wet, stars }}$

- $S M_{g, z}^{\text {major dry, sat }}$

- $S M_{g, z}^{\text {major dry, stars }}$

- $S M_{g, z}^{\text {major wet, sat }}$

- $S M_{g, z}^{\text {major wet, stars }}$

Todas estas variables nos permiten realizar un seguimiento detallado de la historia del ensamblado de las galaxias y la evolución con el redshift de las fracciones de masa estelar aportadas por los diferentes procesos. Además de las variables anteriores, se definieron variables análogas para seguir la evolución de las metalicidades de las galaxias, dadas por la relación de abundancias del contenido de hierro $(\mathrm{Fe})$ e hidrógeno $(\mathrm{H})$ de las estrellas, v.g., cociente $\mathrm{Fe} / \mathrm{H}$. Para cada proceso considerado se hace un seguimiento de las masas de $\mathrm{Fe}$ y de $\mathrm{H}$ recibidas por la galaxia, ya sea por la agregación de estrellas de otras galaxias o por la formación estelar in situ. Por lo tanto, para cada galaxia a un dado valor de redshift (i.e. tiempo), tenemos cantidades $\mathrm{Fe}_{g, z}^{\mathrm{proc}, \text { comp }}$ y $\mathrm{H}_{g, z}^{\text {proc, comp }}$.

Los mecanismos antes mencionados que directamente dan lugar a la formación y acreción de estrellas pueden ser agrupados en "procesos internos" y "procesos externos". Los primeros no involucran intercambio de material con otras galaxias sino que son propios de las galaxias mismas, mientras que los segundos se caracterizan por la presencia de fusión con otras galaxias. Los procesos indicados en los ítems $(i)$ e (ii) son considerados procesos internos. Cabe destacar que el segundo caso referente a la formación estelar debida a inestabilidades de disco, se requiere la presencia de una galaxia cercana que actúe como perturbadora a través de su interacción y debido a ello, se desate la formación estelar del disco que se torna inestable. Por lo que en tal sentido, no sería un proceso interno, estríctamente; sin embargo, el mismo es considerado como tal debido a que el brote de formación estelar consume sólo el gas contenido en la galaxia perturbada 


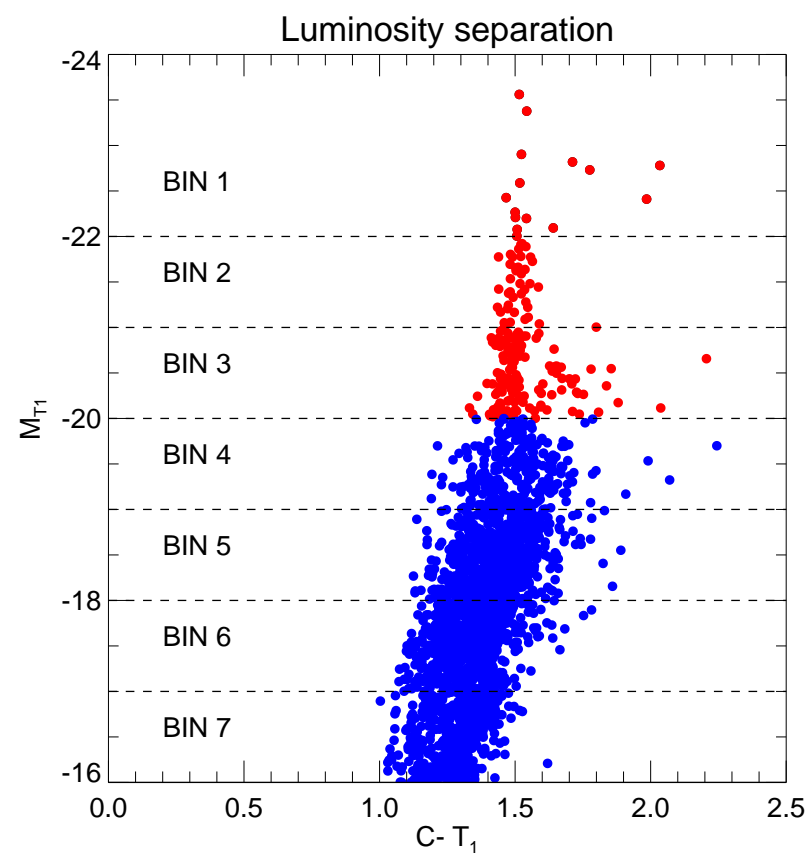

Figura 4.1: RCM del cúmulo $\mathrm{C}_{15} 5_{3}$ ( $g 51$ ), dividida en intervalos de magnitud. Las galaxias del extremo débil se presentan en azul, mientras que las del extremo brillante se muestran en rojo.

que sufre las inestabilidades en el disco. En los procesos externos se produce formación o agregación de estrellas debido a fusiones galácticas donde sí hay incorporación de material perteneciente a las galaxias satélites. Estos procesos son los agrupados en el ítem (iii), que comprende fusiones mayores y menores tanto húmedas como secas.

A fin de alcanzar una mejor visualización de los mecanismos que determinan las propiedades de las galaxias a lo largo de la RCM, dividimos la relación en siete intervalos de magnitud. En particular, utilizamos la RCM simulada en el sistema de Washington: plano color magnitud dado por $\left(C-T_{1}\right)$ vs. $T_{1}$. Si bien en todos los planos color magnitud estudiados en el capítulo 3 puede distinguirse el quiebre de la RCM en el extremo brillante, el mismo resulta más claro en este plano dada la sensibilidad del sistema de Washington a la metalicidad de las galaxias (se puede apreciar la comparación de la RCM en distintos planos en la figura 3.4). En nuestro estudio consideramos seis intervalos desde $M_{\mathrm{T}_{1}}=$ -16 a $M_{\mathrm{T}_{1}}=-22$ de una magnitud de ancho cada uno $\left(\Delta M_{\mathrm{T}_{1}}=1\right)$ y un séptimo intervalo para las galaxias más brillantes de la relación, con un ancho de dos magnitudes $\left(-22<M_{\mathrm{T}_{1}} \leq-24\right)$. Este último intervalo involucra dos magnitudes a fin de mejorar el análisis estadístico dado el bajo número de galaxias extremadamente luminosas en los cúmulos de galaxias simulados. Luego, en lo que sigue, denominaremos como intervalos de magnitud (bin) $=1$ e intervalo (bin) $=7$, a aquéllos correspondientes a los rangos de magnitudes $-24<M_{\mathrm{T}_{1}} \leq-22$ y $-17<M_{\mathrm{T}_{1}} \leq-16$, respectivamente (ver Figura 4.1). Con esta división, analizamos la evolución con el redshift de las fracciones de masa estelar aportadas por diferentes procesos y componentes a las galaxias en un dado intervalo de magnitudes. Así mismo, analizamos la metalicidad promedio de estas contribuciones de masa estelar. 


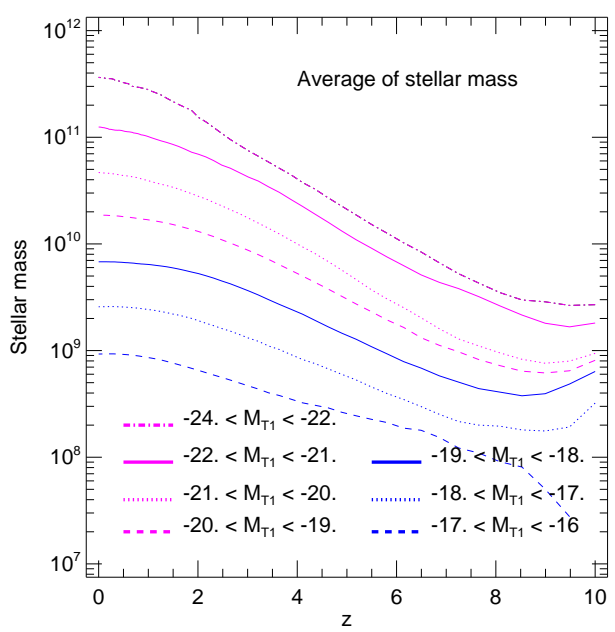

Figura 4.2: Promedio de la masa de la componente estelar de las galaxias en los diferentes intervalos de magnitud de la RCM, en función del redshift. Los promedios se calculan sobre los cúmulos simulados del set C15.

\subsection{Definición de nuevas variables}

Definimos a continuación nuevas variables que se incluyen en nuestros cálculos. La masa total adquirida a redshift $z$ por galaxias que se encuentran en el intervalo de magnitudes $b$, a $z=0$, proveniente de una componente particular ("comp"), aportada por un dado proceso ("proc"), es

$$
S M_{b, z}^{\text {proc, comp }}=\sum_{g=1}^{N_{b, z}} S M_{g, z}^{\text {proc, comp }},
$$

donde $N_{b, z}$ es el número de galaxias dentro del intervalo $b$, en el paso temporal de la simulación caracterizado por el redshift $z$. Luego, considerando la contribución combinada de las dos componentes de masa estelar ("stars" y "sat") aportadas por diferentes procesos que fueron definidas previamente, se obtiene la cantidad

$$
S M_{b, z}^{\text {proc }}=S M_{b, z}^{\text {proc, stars }}+S M_{b, z}^{\text {proc, sat }} .
$$

Finalmente, la masa estelar total acumulada por todas las galaxias en un dado intervalo de magnitudes que resulta de la contribución de todos los procesos involucrados es

$$
S M_{b, z}^{(\mathrm{i})+(\mathrm{ii})+(\mathrm{iii})}=\sum_{\text {proc }=1}^{N_{\text {proc }}} S M_{b, z}^{\text {proc }},
$$

donde $N_{\text {proc }}$ es el número total de procesos considerados en nuestro análisis, los cuales se agrupan en los conjuntos (i), (ii) y (iii) descriptos más arriba.

Con el fin de estudiar la evolución de la metalicidad de las galaxias a lo largo de toda su vida, dada por el cociente $\mathrm{Fe} / \mathrm{H}$, definimos a continuación variables similares a las correspondientes a la masa estelar. Para cada proceso considerado y en cada redshift, el modelo registra la cantidad de masa de hierro e hidrógeno que cada galaxia recibe, proveniente de estrellas agregadas a las galaxias a través de fusiones o bien, estrellas creadas in situ. Así pues, podemos definir las variables $\mathrm{Fe}_{g, z}^{\mathrm{proc}, \mathrm{comp}}$ y $\mathrm{H}_{g, z}^{\mathrm{proc}, \text { comp }}$, correspondientes al Fe y al H, respectivamente. 
La metalicidad promedio pesada por masa de las estrellas adquiridas como resultado de un dado proceso, a un dado redshift, por las galaxias pertenecientes a un cierto intervalo de magnitud en la RCM es

$$
\langle(\mathrm{Fe} / \mathrm{H})\rangle_{b, z}^{\mathrm{proc}, \mathrm{comp}}=\frac{\sum_{g=1}^{N_{b, z}}\left(\frac{F e_{g, z}^{\mathrm{proc}, \mathrm{comp}}}{H_{g, z}^{\text {proc comp }}}\right) S M_{g, z}^{\mathrm{proc}, \text { comp }}}{S M_{b, z}^{\mathrm{proc}, \mathrm{comp}}} .
$$

Dada esta cantidad para cada componente podemos ahora estimar la metalicidad promedio, pesada por masa, de las estrellas adquiridas en un dado proceso, teniendo en cuenta las dos componentes "sat" y "stars". Esto se expresa de la siguiente manera

$$
\begin{aligned}
\langle(\mathrm{Fe} / \mathrm{H})\rangle_{b, z}^{\mathrm{proc}} & =\frac{\langle(\mathrm{Fe} / \mathrm{H})\rangle_{b, z}^{\mathrm{proc}, \text { stars }} S M_{b, z}^{\text {proc }, \text { stars }}}{S M_{b, z}^{\text {proc }}} \\
& +\frac{\langle(\mathrm{Fe} / \mathrm{H})\rangle_{b, z}^{\text {proc }, \text { sat }} S M_{b, z}^{\text {proc }, \text { sat }}}{S M_{b, z}^{\text {proc }}} .
\end{aligned}
$$

Luego, el promedio pesado por masa, de todos los procesos considerados resulta

$$
\langle(\mathrm{Fe} / \mathrm{H})\rangle_{b, z}^{(\mathrm{i})+(\mathrm{ii})+(\mathrm{iii})}=\frac{\sum_{\mathrm{proc}=1}^{N_{\text {proc }}}\langle(\mathrm{Fe} / \mathrm{H})\rangle_{b, z}^{\mathrm{proc}} S M_{b, z}^{\text {proc }}}{S M_{b, z}^{(\mathrm{i})+(\mathrm{ii})+(\mathrm{iii})}} .
$$

En estas tres últimas definiciones hemos hecho uso de las cantidades dadas por las ecuaciones $4.1,4.2$ y 4.3 .

Las dos siguientes secciones se enfocan en el análisis de la evolución de las fracciones de masa resultantes de los procesos responsables de la fomación y acreción de masa estelar, y de la evolución de las correspondientes metalicidades.

\subsection{Evolución de las fracciones de masa}

Utilizando las definiciones dadas en la sección anterior estimamos la fracción de masa estelar agregada a las galaxias en cada intervalo de magnitudes, para un dado valor de $z$, proveniente del aporte de cada uno de los procesos considerados. Distinguimos entre las dos posibles componentes "sat" y "stars" definidas previamente, las fracciones se definen como

$$
\mathrm{SMFrac}_{b, z}^{\text {proc, comp }}=\frac{S M_{b, z}^{\text {proc, comp }}}{S M_{b, z}^{(\mathrm{i})+(\mathrm{ii})+(\mathrm{iii})}} .
$$

Agruparemos estas fracciones de masas estelares de acuerdo con los aspectos que deseamos analizar.

Comenzamos por considerar las fracciones de la masa estelar de las estrellas recientemente formadas, por un lado, y la masa de estrellas que provienen de las galaxias satélites acretadas, por el otro. Hacemos esto sin distinguir que proceso particular aporta a cada una de las componentes. Tenemos, entonces, 


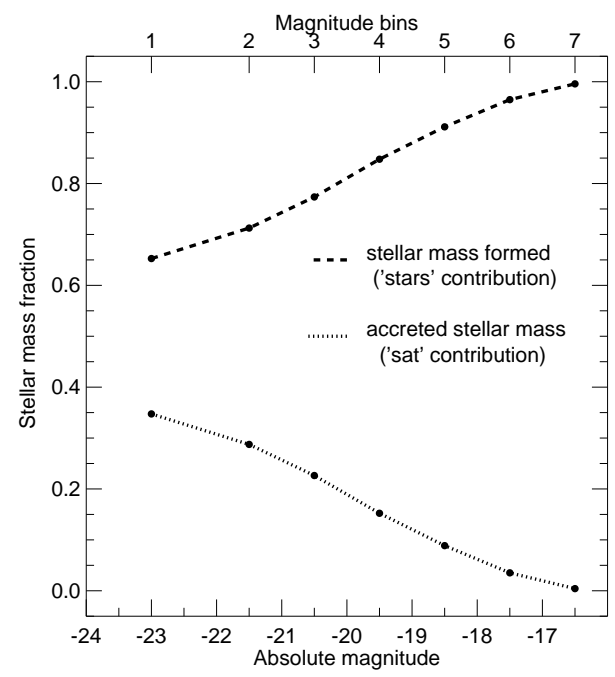

Figura 4.3: Fracciones de masa estelar resultantes de los aportes de las componentes "stars" y "sat" acumulados a $z=0$ por las galaxias de los cúmulos C15, para los diferentes intervalos de magnitud definidos.

$$
\operatorname{SMFrac}_{b, z}^{\text {comp }}=\sum_{\text {proc }=1}^{N_{\text {proc }}} \operatorname{SMFrac}_{b, z}^{\text {proc, comp }}
$$

para las componentes "stars" y "sat". La dependencia de estas fracciones con el intervalo de magnitudes de la RCM en el cual se encuentran las galaxias se presenta en la figura 4.3 para los cúmulos C15. Como puede verse, la masa estelar de todas las galaxias que forman la relación, está principalmente dada por la componente "stars", la cual varía desde un 95 por ciento para las galaxias menos luminosas $\left(M_{T_{1}} \geq-17\right.$, en el intervalo de magnitudes intervalo 7 ) a un $\sim 65$ por ciento para las galaxias más luminosas $\left(M_{T_{1}} \leq-24\right.$, intervalo 1). Esta reducción gradual de la fracción de masa aportada por la componente "stars" para las galaxias más luminosas ocurre como consecuencia de la agregación de estrellas provenientes de galaxias satélites, indicando que las fusiones entre galaxias se vuelven más importantes para las galaxias más masivas, y que estas fusiones son principalmente secas, es decir, con poco contenido de gas en el remanente, lo cual restringe la formación estelar in situ. Esta inferencia se vuelve evidente en el análisis detallado que presentamos a continuación.

Analizamos ahora la evolución con el redshift de las contribuciones acumuladas de masa estelar, aportadas por los distintos procesos considerados (conjuntos $i$, ii, iii). Discriminamos en diferentes intervalos de magnitud de la RCM. La evolución de las correspondientes fracciones medias de masa estelar se muestra en las figuras 4.4 y 4.5 para galaxias con magnitudes en las intervalos 1, 3 y 6, en los cúmulos C15 y C14, respectivamente. Estas fracciones de masa (valores medios de cada proceso en cada intervalo de magnitud) están dadas respecto a la contribución total a la masa estelar de las galaxias en cada redshift considerado. Si bien hemos tenido en cuenta tanto las componentes "stars" como "sat" provenientes de fusiones galácticas mayores secas, hemos encontrado que la contribución de las estrellas formadas in situ (componente "stars") a la masa total de las galaxias es despreciable. Por lo tanto, en lo que sigue simplemente nos referiremos a este proceso como fusión galáctica mayor seca, indicando que sólo se está considerando la masa estelar proveniente de la componente "sat" de dicho proceso.

De la inspección de estos gráficos vemos que la formación estelar en modo pasivo 

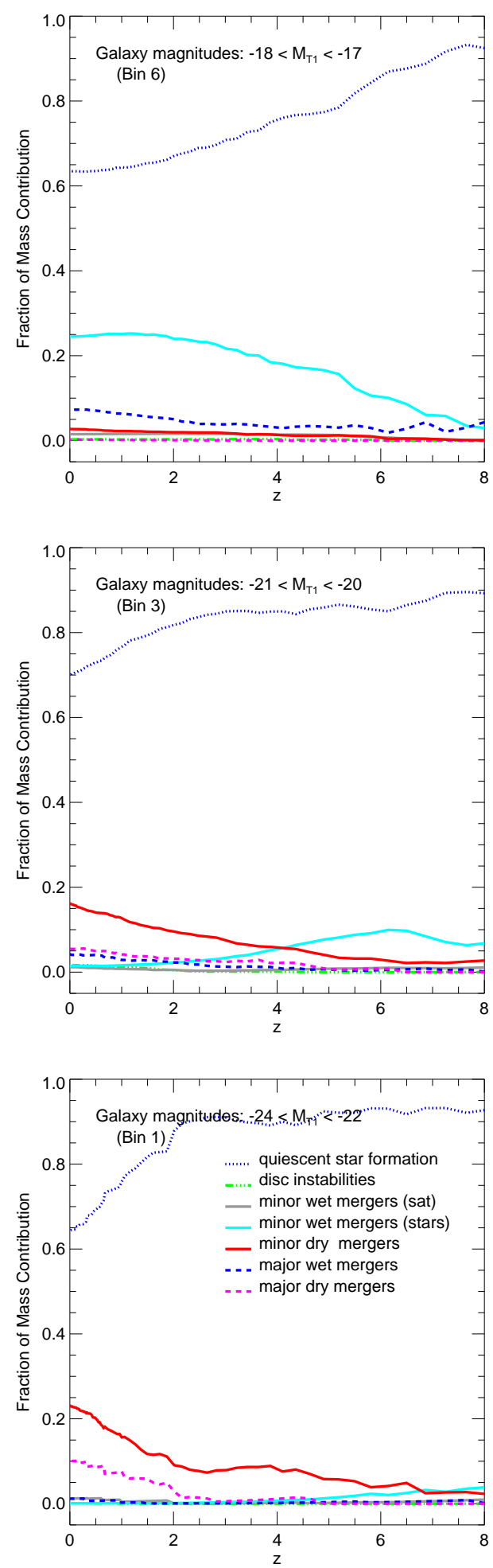

Figura 4.4: Evolución de las fracciones de masa estelar media de los cúmulos C15 debidas a los diferentes procesos de formación y agregación de estrellas considerados. Se muestran las fracciones de masa de las galaxias de los intervalos de magnitud 1,3 y 6 , normalizados respecto del aporte total de masa estelar para cada redshift. Panel Superior: Galaxias con magnitudes en el rango $-18<M_{T_{1}} \leq-17$ (intervalo 6). Panel del medio: Galaxias con magnitudes en el rango $-21<M_{T_{1}} \leq-20$ (intervalo crítico 3). Panel Inferior: Galaxias con magnitudes en el rango $-24<M_{T_{1}} \leq-22$ (intervalo 1 .) 

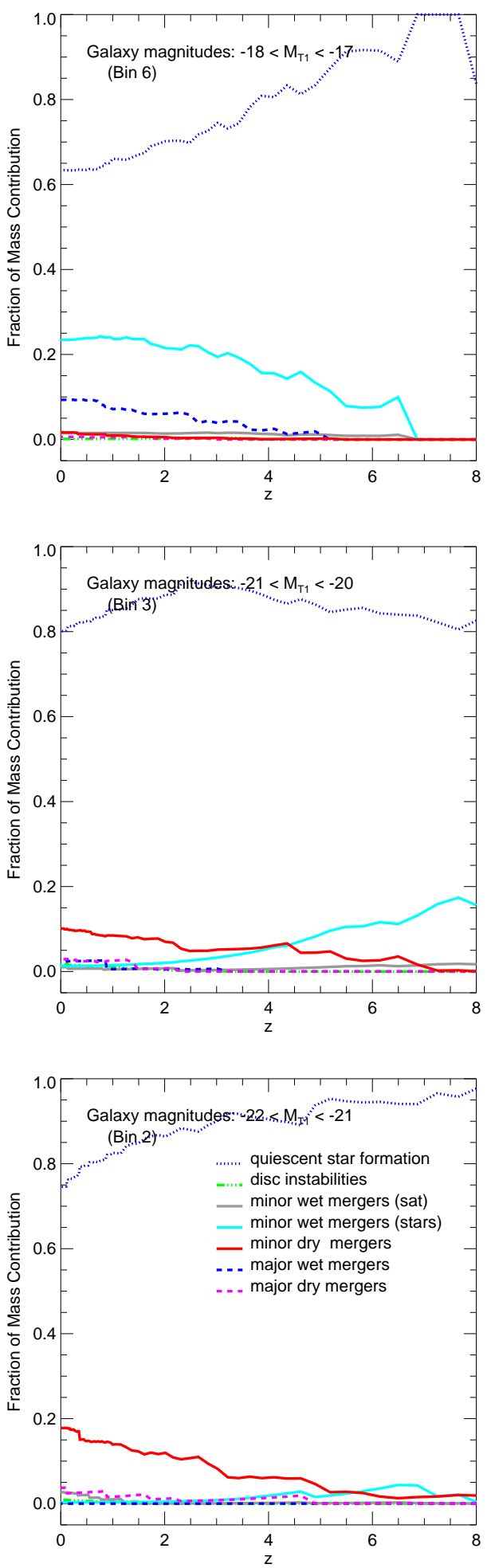

Figura 4.5: Evolución de las fracciones de masa estelar media de los cúmulos C14 debidas a los diferentes procesos de formación y agregación de estrellas considerados. Se muestran las fracciones de masa de las galaxias de los intervalos de magnitud 2, 3 y 6 , normalizados respecto del aporte total de masa estelar para cada redshift. Panel Superior: Galaxias con magnitudes en el rango $-18<M_{T_{1}} \leq-17$ (intervalo 6). Panel del medio: Galaxias con magnitudes en el rango $-21<M_{T_{1}} \leq-20$ (intervalo 3). Panel Inferior: Galaxias con magnitudes en el rango $-22<M_{T_{1}} \leq-21$ (intervalo 2 ). 
aparece como el proceso dominante que contribuye a la masa estelar de las galaxias en todos los intervalos de magnitudes a todo redshift (i.e. tiempo). Sin embargo, podemos ver que la fracción de masa estelar correspondiente a este proceso decrece monótonamente a redshifts decrecientes a medida que el reservorio de gas frío de las galaxias se va agotando. La contribución relativa del resto de los procesos, agrupados en inestabilidades de disco y fusiones galácticas, cambia a medida que nos movemos a lo largo de la RCM. El panel superior de la figura 4.4 se muestra la fracción media de masa para galaxias en el intervalo de magnitud correspondiente a luminosidades bajas $\left(-18<M_{T_{1}} \leq-17\right.$, intervalo 6). Allí puede apreciarse claramente que la segunda contribución más importante a la masa estelar proviene de las estrellas formadas durante fusiones menores húmedas, es decir, de la componente "stars" de las fusiones galácticas menores húmedas. Esta fracción de masa aumenta al disminuir el redshift, alcanzando un valor del 25 por ciento a $z=0$. Estrictamente, este valor del 25 por ciento se alcanza ya a $z=2$, indicando que las fusiones menores húmedas no juegan un rol importante desde esa época, ya que estamos considerando cantidades acumuladas. La siguiente contribución en importancia para estas galaxias es aquélla correspondiente a las fusiones galácticas mayores húmedas. Las contribuciones provenientes de inestabilidades de disco y otros tipos de fusiones galácticas son despreciables para las galaxias poco luminosas.

A medida que nos movemos a luminosidades mayores en la RCM, la situación cambia notablemente. Las fracciones de masa en el intervalo de magnitudes crítico, donde ocurre la separación de la RCM respecto del ajuste lineal, se muestran en el panel intermedio de la figura 4.4. A estas magnitudes, la contribución más relevante a la masa estelar (luego de la formación estelar pasiva) proviene de las fusiones galácticas menores de tipo seco, mientras que la relevancia de la contribución de la componente "stars" de las fusiones galácticas menores húmedas, decrece considerablemente desde $z \sim 4$ al presente, volviéndose despreciable a $z=0$. Esto muestra un fuerte contraste con las relevancias de los procesos observados más arriba para las galaxias menos luminosas (intervalo 6). Nótese que en el intervalo crítico aparece también una contribución secundaria, a bajo redshift proveniente de fusiones galácticas tanto mayores húmedas como fusiones mayores secas.

Todas estas fusiones galácticas consideradas en conjunto son responsables de aproximadamente el 20 por ciento de la masa estelar recibida por las galaxias correspondientes al intervalo de magnitudes $-21<M_{T_{1}} \leq-20$ (intervalo crítico) a $z=0$.

Para las galaxias más luminosas $\left(-24<M_{T_{1}} \leq-22\right.$, (intervalo 1), la importancia de la contribución a la masa estelar de las fusiones galácticas menores secas es aun más importante que la observada en el intervalo crítico, ganando importancia ya desde épocas más tempranas $(z \approx 6)$ y alcanzando su valor máximo de $\sim 22$ por ciento en $z=0$. La contribución de las fusiones galácticas mayores secas también se incrementa notablemente desde $z \approx 2$ llegando a una fracción de $\approx 10$ por ciento en $z=0$. Por lo tanto, la contribución conjunta de estos dos procesos en la época actual alcanza un porcentaje de $\approx 32$ por ciento, consistente con el resultado mostrado en la figura 4.3.

Si bien la descripción previa está hecha sobre la base de las fracciones de masa medias de las galaxias en los cúmulos C15, en los cuales se observa claramente el cambio de pendiente en la RCM, resultados similares se muestran en la figura 4.5 para el caso de los cúmulos más pequeños, C14. En estos últimos, sin embargo, la estadística se vuelve muy pobre a grandes luminosidades, con sólo unas pocas (o ninguna) galaxias en el intervalo de mayores luminosidades (por ello en el panel superior de la figura 4.5 se muestra el intervalo 2 en lugar del 1). Este comportamiento está de acuerdo con el escenario inferido por Martínez et al. (2010) en base a estudios de galaxias en grupos y en el núcleo de cúmulos emisores de rayos $\mathrm{X}$, en el cual establecen que estas galaxias pueblan casi la misma relación color magnitud. $\mathrm{Su}$ resultado da validez a trabajos anteriores que indican que la relación color magnitud es independiente de la sobredensidad del ambiente (Hogg et al., 2004; López-Cruz et al., 2004). 


\subsubsection{Influencia de la resolución numérica}

Como puede apreciarse en la figura 4.4, la contribución de las fusiones galácticas menores, a la masa estelar, es relevante para las galaxias de todos los intervalos de magnitud. El cociente de masas de las fusiones galácticas menores que son resueltas por el modelo semianalítico SAG depende de la masa de las galaxias involucradas. Dada la resolución de los halos de materia oscura de la simulación de $N$-cuerpos/SPH utilizada como base, un límite superior conservador para la resolución de masa de las galaxias sería $\sim 10^{9} h^{-1} M_{\odot}$ (i.e. la mínima masa de halo resuelta multiplicada por la fracción bariónica). Sin embargo, dada la compleja interacción de los procesos considerados en el modelo semianalítico como el enfriamiento del gas, feedback y la estrangulación (ver capítulo 2), encontramos que nuestro modelo produce objetos con masas estelares tan pequeñas como $1.5 \times 10^{5} h^{-1} M_{\odot}$. Por lo tanto, el cociente de masas mínimo resuelto por el modelo es $\sim 10^{-7}$, correspondiente a la fusión galáctica de un objeto de ese tipo con la galaxia más brillante de un cúmulo - Brightest Cluster Galaxy, BCG - de masa estelar $\simeq 1.5 \times 10^{12} h^{-1} M_{\odot}$ en nuestras simulaciones. Sin embargo, es muy probable que para masas tan pequeñas nuestro modelo sea incompleto y por lo tanto la mínima masa límite sea mayor.

Comparando la función de luminosidad que resulta del SAG con una función de luminosidad observada (v.g. DePropris et al. 2003) se obtiene que nuestro modelo es completo para galaxias con magnitudes absolutas menores a -15 en la banda $b_{J}$, como se puede ver en la figura 2.5. Esto corresponde a galaxias con una masa estelar media de $\simeq 2 \times 10^{8} h^{-1} M_{\odot}$. Consecuentemente, para el caso de las galaxias BCG esperamos que nuestro modelo sea completo en fusiones con cocientes de masas mayores a $10^{-4}$. Para las galaxias en los cúmulos C15, los cocientes de masas mínimos resueltos por el modelo son del orden de $4 \times 10^{-5}, 10^{-4}$ y $6 \times 10^{-3}$, para las galaxias en los intervalos de magnitud 1, 3 y 6 , respectivamente. Estos cocientes aumentan a medida que uno considera galaxias menos luminosas. En el caso de las galaxias más luminosas de los cúmulos $C 14$ (i.e. intervalo 1 ), el cociente de masas mínimo es mayor, $\sim 2,5 \times 10^{-5}$, pero del mismo orden de magnitud que en el caso anterior.

En general, el número de fusiones que sufren las galaxias a lo largo de su historia aumenta de una manera bastante monótona a medida que se consideran fusiones de galaxias con cocientes de masa menores. Esto puede verse en la figura 4.6, donde se presentan los resultados para galaxias de los cúmulos $C 15_{3}$ (g51) (panel superior) y $C 14_{1}(g 1542)$ (panel inferior). En el primer caso se consideran las galaxias contenidas en los intervalos de magnitud 1, 3 y 6, mientras que para el cúmulo menos masivo las galaxias son agrupadas según su pertenencia a los extremos débil y brillante de la RCM. Las fusiones menores (definidas como aquéllas con cocientes de masa menores a 0,3 , ver capítulo 3), son dominantes para todas las galaxias de la RCM independientemente de la masa del cúmulo. El mayor número de galaxias del cúmulo más masivo permite analizar la situación por intervalos de magnitud, a partir de lo cual se observa que, para el intervalo con galaxias de menor luminosidad, el máximo número de fusiones no se alcanza para el menor valor del cociente de masas, como ocurre en el caso de las galaxias más luminosas. Este hecho refleja la influencia del límite en la resolución de masa de las simulaciones. Cada uno de estos intervalos de magnitud contiene un número diferente de galaxias, como se indica en el gráfico, siendo mayor en el caso de los intervalos menos luminosos. Teniendo en cuenta este aspecto, el número promedio de fusiones es mayor para las galaxias más luminosas. Este resultado respalda la conclusión obtenida a partir del análisis de la figura 4.3, donde se aprecia un aumento gradual del dominio de la componente "sat" en el aporte de masa estelar para galaxias más luminosas. 

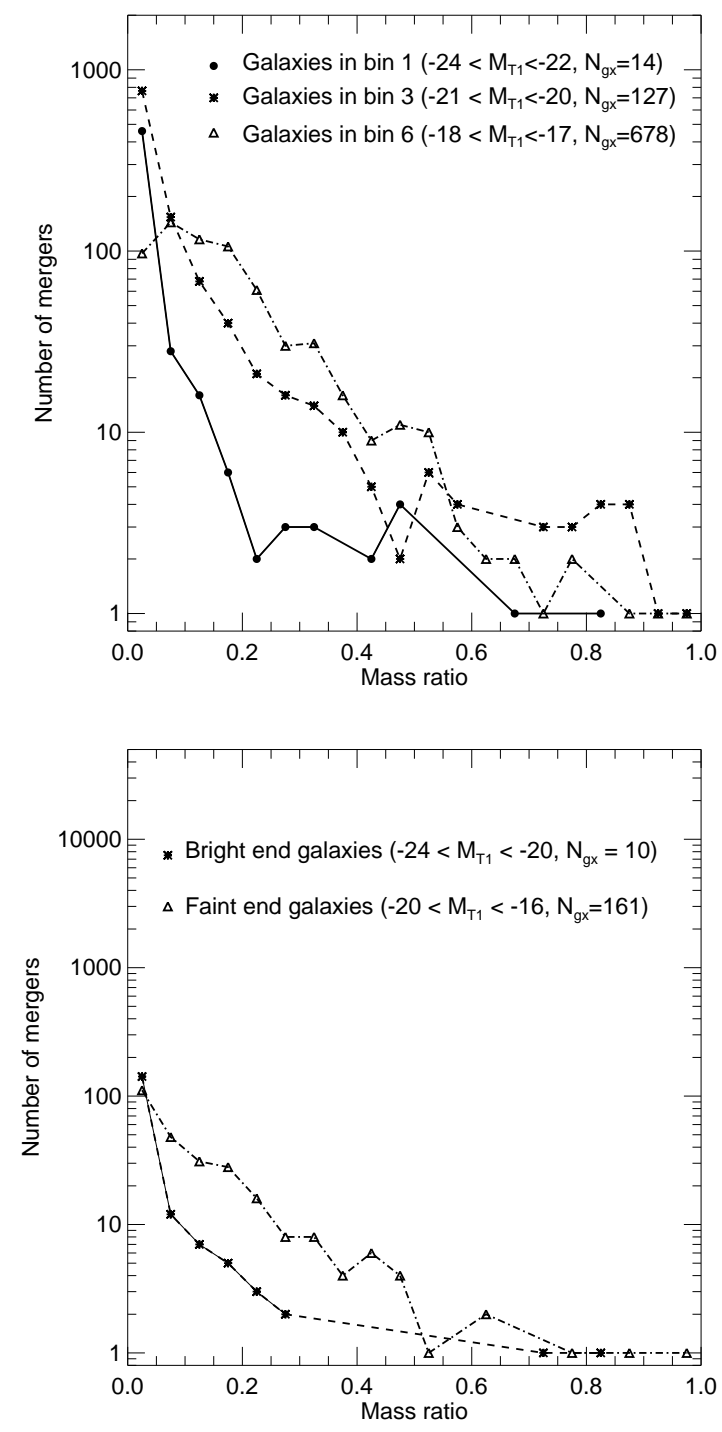

Figura 4.6: Número de fusiones galácticas sufridas por las galaxias del cúmulo $\mathrm{C} 15_{3}$ (g51) (panel superior) y C14 1 (g1542) (panel inferior) en toda su vida, en función del cociente de masa bariónica de las galaxias que se fusionan. Las diferentes curvas corresponden a galaxias contenidas en los intervalos de magnitudes 1,3 y 6 en el caso del cúmulo $\mathrm{C} 15_{3}$, y en los extremos débil y brillante en el caso del cúmulo $\mathrm{C} 14_{1}$. 


\subsubsection{Influencia del umbral de la fracción de gas}

Del análisis de las fracciones de masa estelar discutido en las secciones anteriores es claro que la evolución de las galaxias del extremo brillante de la RCM (i.e. aquellas más brillantes que el valor crítico: $M_{T_{1}} \approx-20$ ), es debida a la contribución seca tanto de las fusiones mayores como menores. Por el otro lado, las galaxias más débiles son afectadas por la componente húmeda de las fusiones.

Estas conclusiones están de acuerdo con el trabajo de Skelton et al. (2009), quienes encontraron que la mayoría de las galaxias del extremo brillante de la RCM (galaxias elípticas de tipo temprano) han sufrido fusiones secas, en contraste con lo que ocurre para las galaxias más débiles. Sus resultados están basados en un modelo simplificado que aisla los efectos de las fusiones secas en los colores de la RCM. El historial de fusiones y las fracciones de gas de las galaxias fueron extraidos del modelo semianalítico de Somerville et al. (2008). Este modelo es capaz de reproducir el cambio de pendiente de la secuencia roja, tal como se observa en las galaxias elípticas de tipo temprano del Sloan Digital Sky Survey (SDSS). Skelton et al. (2009) sostienen que este cambio en la pendiente y la magnitud a la cual ocurre dicho quiebre, dependen fuertemente del umbral de fracción de gas elegido, por debajo el cual las fusiones galácticas son consideradas secas. Este umbral varía entre un 10 y un 30 por ciento.

En nuestro modelo, por el contrario, la inclinación de la RCM hacia colores más azules tiene sólo una dependencia débil con la elección del umbral de fracción de gas elegido para distinguir entre fusiones húmedas y secas $\left(f_{\text {gas,minor }}\right.$ and $f_{\text {gas,major }}$, tal como fueron definidos previamente en el capítulo 2). El parámetro $f_{\text {gas,major no posee ninguna }}$ influencia en el efecto de las fusiones mayores. Esto es debido a que esas fusiones siempre dan lugar a la formación de un esferoide y al agotamiento del gas frío disponible en un brote de formación estelar, sin importar la cantidad de gas disponible en las galaxias fusionadas. En este sentido, el parámetro $f_{\text {gas,major }}$ sólo tiene como objetivo la clasificación de las fusiones mayores entre húmedas y secas, afectando solamente los porcentajes de las componentes "stars" y "sat" aportadas por estos procesos a la masa estelar de las galaxias. Hemos verificado que variando este umbral en el rango $0,2 \leq f_{\text {gas,major }} \leq 0,6$ no se obtienen cambios significativos con respecto a los resultados presentados en la figura 4.4, los cuales fueron obtenidos utilizando $f_{\text {gas,major }}=0,4$. Por otro lado, el valor $f_{\text {gas,minor }}=0,6$, utilizado para las fusiones menores ha sido ajustado junto al resto de los parámetros libres del modelo para reproducir varias de las propiedes observadas de las galaxias, tal como fuera descripto en el capítulo 3.

Es importante enfatizar que nuestro modelo no ha sido construido con el fin de producir el quiebre en la RCM, sino que éste surge naturalmente como consecuencia de los procesos físicos incluídos en el modelado. En particular, alterando el valor $f_{\text {gas,major }}$ dentro del rango mencionado previamente, siempre aparece un quiebre en el extremo brillante de las RCM simuladas. Esto ocurre sin un cambio apreciable en el valor de la pendiente y la magnitud crítica. En este sentido nuestros resultados difieren de aquéllos obtenidos por Skelton et al. (2009), quienes afirman encontrar una fuerte dependencia del quiebre con el valor adoptado del umbral para la fracción de gas. Más aun, nosotros no encontramos una dependencia significativa tampoco, en los resultados presentados por ellos (ver la figura 1 de Skelton et al. (2009)). En cualquier caso, tanto nuestros resultados como los obtenidos por Skelton et al. (2009) dan un fuerte sustento al hecho de que las componentes secas de las fusiones galácticas son relevantes para la formación de las galaxias más brillantes de la RCM, tanto en cúmulos como para galaxias de campo. Se ha sugerido que las fusiones secas sólo aumentan la masa de las galaxias sin alterar sus colores debido a la ausencia de una formación estelar asociada a estas fusiones, causando entonces la separación del extremo brillante de la RCM, respecto de la tendencia lineal observada a menores luminosidades (Bernardi et al. 2007; Skelton et al. 2009). El análisis de la evolución de las metalicidades de las estrellas formadas y agregadas es fundamental para explicar esta afirmación. Parte de los resultados referentes a las metalicidades se presentan en la siguiente sección, y dan lugar a un estudio más exhaustivo que se detalla 
en el siguiente capítulo.

\subsection{Evolución de la metalicidad}

Con el fin de analizar la evolución de la metalicidad de las galaxias con el redshift y su dependencia con el intervalo de magnitudes en el que las mismas se encuentran a $z=0$, se estima el promedio pesado por masa de la abundancia de hierro de las estrellas. Para ello usamos la ecuación 4.6. Mediante la misma, podemos calcular el promedio pesado de la abundancia de hierro de las estrellas resultante de la contribución de todos los procesos, tanto internos como externos (conjuntos $i$, ii, iii). Estas cantidades se calculan para las galaxias en cada intervalo de magnitud $b$, en un dado tiempo $z$. La misma ecuación nos permite identificar las contribuciones a la metalicidad de las galaxias de los procesos internos y externos, considerando separadamente los conjuntos $(i, i i)$ e (iii), respectivamente.

El cálculo de las metalicidades medias se realiza para las estrellas pertenecientes a las galaxias de los cúmulos C14 y C15, por separado. La evolución de los valores medios de la metalicidad de galaxias que a $z=0$ se encuentran en un dado intervalo de magnitudes en la RCM, se muestra en las figuras 4.7 y 4.8 para los cúmulos C15 y C14, respectivamente. En el panel superior de estas dos figuras podemos ver las abundancias de la componente estelar resultante del contenido de hierro de las estrellas generadas y/o acretadas en todas las fusiones galácticas. La metalicidad crece a medida que el redshift decrece. El valor final alcanzado a $z=0$ para las galaxias en los diferentes intervalos de magnitud corresponde a valores de metalicidad subsolares, tanto para los cúmulos C15 como C14, siendo mucho menores en este último caso, como consecuencia de la menor metalicidad de las galaxias satélites que estos cúmulos acretan. En el panel del medio de ambas figuras se presenta la abundancia media de hierro originada como resultado de la acción de procesos internos, es decir, debida a la formación estelar pasiva y a aquélla proveniente de eventos de inestabilidad de disco. En este caso, el crecimiento de la metalicidad es suave y monótono, a medida que disminuye el redshift. Esto es así para los diferentes intervalos de magnitud, alcanzando valores finales que superan la metalicidad solar en el intervalo más luminoso, tanto para los cúmulos C15 como C14. En ambos grupos de cúmulos puede verse que el rango de metalicidad final alcanzado por las galaxias en los intervalos más luminosos, por encima del quiebre de la RCM $\left(M_{\mathrm{T} 1}>-20\right)$, es mucho más angosto $(\approx 0,15-0,2$ dex $)$, comparado con al rango de metalicidades finales de las galaxias de menor luminosidad. Este comportamiento es más pronunciado para las galaxias del cúmulo C15. En el panel inferior de las figuras se muestra la metalicidad media total alcanzada por las galaxias de cada intervalo de magnitud, donde se ha considerado la contribución de todos los procesos internos y externos. Del análisis de la contribución de estos procesos por separado se ve claramente que el comportamiento de la metalicidad media total está principalmente determinado por los procesos internos. Al promediar la metalicidad media resultante de estos procesos con las abundancias proporcionadas por los procesos externos, resulta que estos últimos contribuyen a reducir la metalicidad total que caracteriza a las galaxias en los distintos instantes de tiempo.

Deseamos enfatizar una característica notable que se pone en evidencia estos gráficos y que resulta sumamente relevante para este estudio; las metalicidades finales que alcanzan las galaxias más luminosas, con magnitudes que están por encima del quiebre del RCM, no difieren demasiado entre sí, sino que comprenden un rango pequeño en relación a las metalicidades finales alcanzadas por las galaxias menos luminosas de la RCM. Este fenómeno está directamente relacionado con la similitud de colores que presentan las galaxias luminosas, llevándolas a separarse de la tendencia lineal general de la RCM.

Otra característica importante que extraemos del análisis previo es que las abundancias químicas medias totales parecen mantener un valor casi constante desde $z=1$ en adelante, especialmente para las galaxias en el extremo brillante. Para las galaxias menos luminosas se detecta un aumento suave de la metalicidad como consecuencia de la 

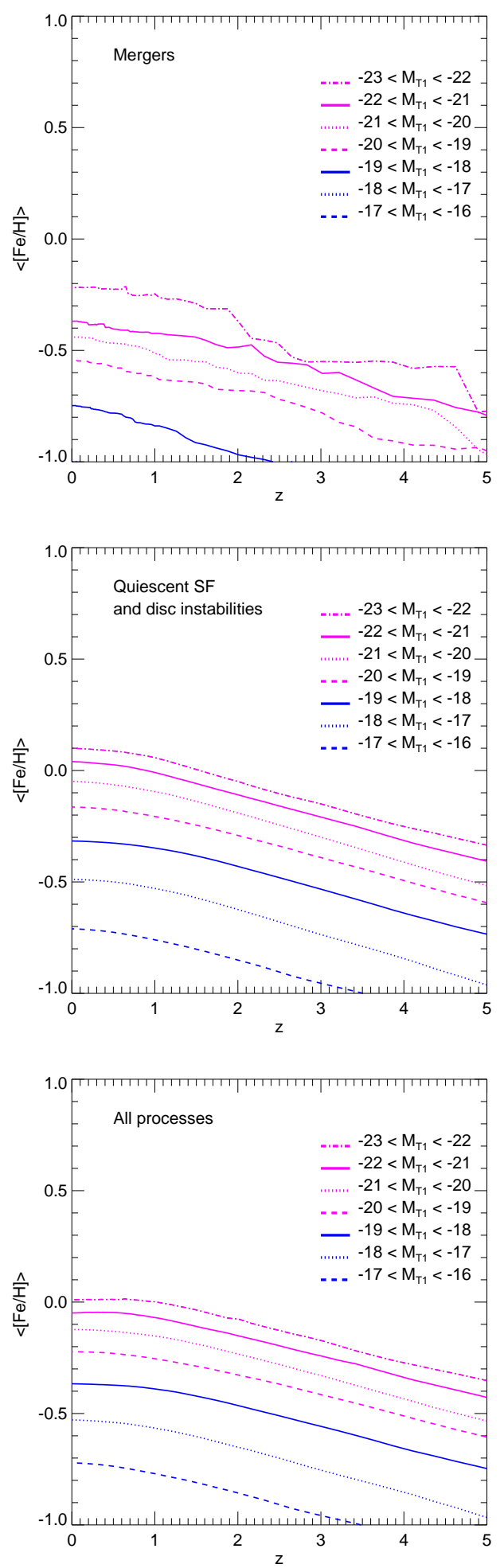

Figura 4.7: Evolución con el redshift de la abundancia media de hierro de la componente estelar, de las galaxias que a $z=0$, forman parte de la RCM en un dado intervalo de magnitud. Los promedios se estiman utilizando las galáxias de los cúmulos C15. Panel superior: Metalicidad de la masa estelar acretada y/o generada en fusiones galácticas. Panel medio: Metalicidad debida a la componente estelar generada por los procesos internos de las galaxias, v.g. formación estelar pasiva e inestabilidades de disco. Panel inferior: Metalicidad resultante de la combinación de todos los procesos de formación estelar incluídos en SAG . 

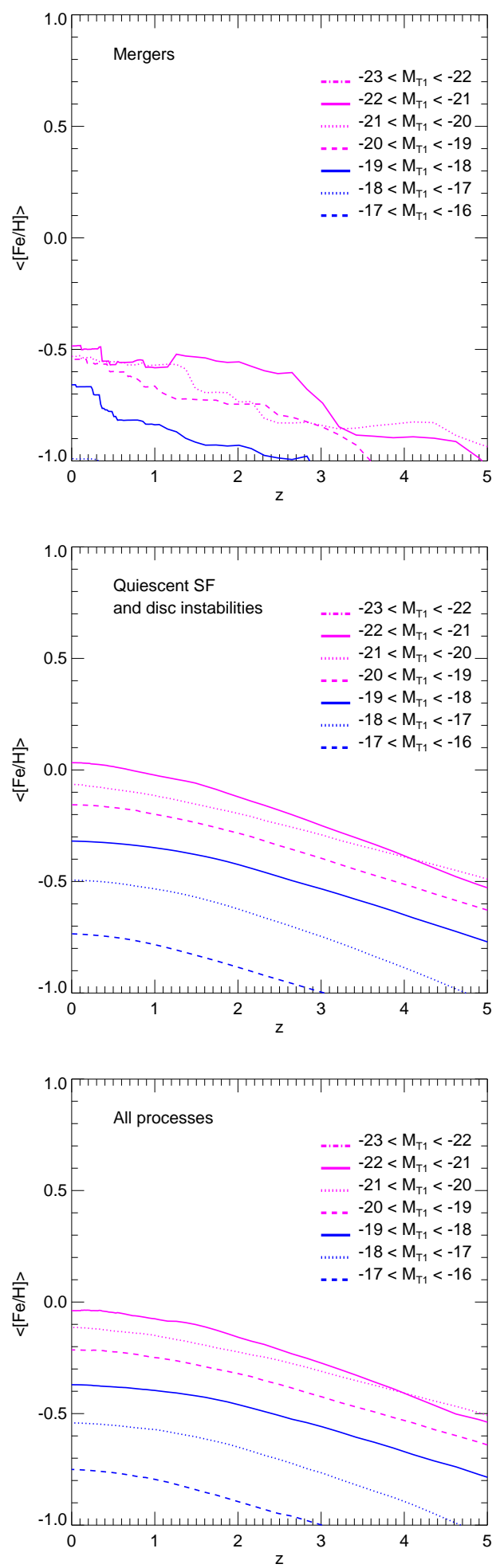

Figura 4.8: Evolución con el redshift de la abundancia media de hierro de la componente estelar de las galaxias de los cúmulos C14, separando la contribución de procesos internos y externos, y considerando la acción conjunta de los mismos, como en la figura 4.7. Los diferentes tipos de líneas representan el valor medio obtenido para las galaxias dentro de los diferentes intervalos de magnitud. 
formación estelar a partir de gas químicamente enriquecido, este fenómeno continúa a bajos redshifts.

Es interesante notar que este resultado es sustentado por observaciones de cúmulos de galaxias a alto redshift que muestran que la pendiente y la dispersión de la RCM, correspondiente a galaxias de tipo temprano seleccionadas morfológicamente, presenta poca o casi ninguna señal de evolución desde $z \approx 1,2$ al presente (Blakeslee et al., 2003; Mei et al., 2006; Jaff et al., 2010). 


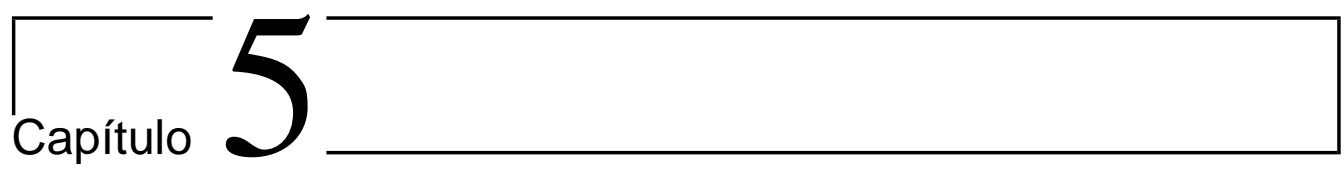

\section{Factores que determinan el quiebre de la RCM}

En el capítulo anterior se analizó la importancia relativa de las fracciones de masa estelar contribuida a las galaxias, en diferentes intervalos de magnitud de la RCM, por los diferentes procesos internos y externos de evolución, considerados en el modelo semianalítico. Se investigó la dependencia con el redshift de estas fracciones, como asi también, la dependencia de la metalicidad asociada a las respectivas componentes estelares, tanto las generadas dentro de cada galaxia como las acretadas durante los eventos de fusión. A partir de este análisis se desprende que la evolución de las galaxias en el extremo brillante de la RCM es conducido por la contribución seca tanto de fusiones menores como mayores. Esta situación ya se encuentra establecida en $z \approx 1$. Los valores finales (a $z=0$ ) de metalicidad de las galaxias en el extremo brillante no cambian desde este momento (redshift). Teniendo en cuenta este hecho, resulta interesante evaluar las caraterísticas de la masa estelar acumulada desde $z=1$ al presente, a fin de poder explicar claramente la razón física de la presencia del quiebre de la RCM. Este es el objetivo de discusión de este capítulo. La validéz de los resultados obtenidos se basa en el buen acuerdo existente con aspectos observacionales inherentes a este análisis.

\subsection{Consideraciones respecto de las variables introducidas en SAG}

A fin de evaluar la influencia de la masa estelar acumulada en las galaxias desde $z=1$ al tiempo presente, en las propiedades de las mismas a $z=0$, consideramos las masas estelares aportadas por las componentes "stars" y "sat" de los procesos de de formación estelar internos (formación estelar pasiva y por inestabilidad de disco) y externos (brotes estelares y agregación de masa estelar de los sistemas satélites durante las fusiones), y los referimos a la masa estelar total alcanzada por las galaxias en $z=0$. Para realizar una correcta interpretación de los resultados que se obtienen de esta estimación, se debe tener en cuenta que la masa estelar actual (en $z=0$ ) de las galaxias es el resultado de la evolución completa seguida por el código semianalítico, incluyendo su reducción como consecuencia del reciclado de materia debido a la evolución estelar. Sin embargo, por construcción, las variables introducidas en SAG que hemos utilizado en el capítulo anterior para analizar las fracciones de masas mostradas en las figuras 4.3 y 4.4, así como las que se usarán en los cálculos que se detallarán en este capítulo, guardan la información de las estrellas generadas y/o agregadas en una galaxia durante procesos de formación estelar internos y externos, pero no tienen en cuenta la disminución de la masa estelar como consecuencia del reciclado de masa producido por la evolución estelar. Este hecho 
afecta especialmente a la masa estelar contribuida por las nuevas estrellas formadas, es decir, a la componente "stars" como se muestra a continuación.

Con el propósito de evaluar el impacto de la falta de consideración de reciclado de masa estelar en las variables "stars" y "sat", en los resultados de fracciones de masa acumulada desde un cierto redshift, estimamos el tiempo de vida de las estrellas formadas en distintos rangos de masa estelar, mediante el uso de un modelo de evolución estelar. De esta manera, obtenemos información sobre la fracción de masa estelar formada en distintos instantes de tiempo que sobrevive al día de hoy. La fracción de estrellas formadas $N(m)$ dentro de un rango de masas $(m, m+d m)$ está dada por la función inicial de masa. El modelo SAG utlizado en esta investigación adopta la función inicial de masa de Salpeter, tal como se detalla en el capítulo 2. La misma puede expresarse como

$$
N(m)=\mathbb{C} m^{-2,35}
$$

Multiplicando esta expresión por la masa $m$ encontramos la distribución $M(m)$, función que da el porcentaje de masa de estrellas formadas dentro de un determinado rango de masas, esto es

$$
M(m) d m=N(m) m d m=\mathbb{C} m m^{-2,35}=\mathbb{C} m^{-1,35} d m
$$

Normalizando esta función y tomando como límites de integración los extremos del rango de masas $(0,1-100) M_{\odot}$, la constante $\mathbb{C}$ y la función $M(m)$ resultan

$$
\begin{gathered}
\mathbb{C}=0,171636 \\
M(m) d m=0,171636 m^{-1,35} d m .
\end{gathered}
$$

Por otro lado, consideramos el modelo de evolución estelar dado por Pietriferni et al. (2004). En su trabajo, los autores proveen una base de datos que contiene una grilla de tracks evolutivos que se encuentra publicada en BASTI (Bag of Stellar Tracks and Isochrones). La tabla 5.1 reproduce un fragmento de la tabla en BASTI. Los valores obtenidos por el modelo de evolución estelar y presentados en la tabla 5.1 están caracterizados por una abundancia de helio $Y=0,256$ y por una metalicidad total $Z=0,008$. Esta última cantidad es equivalente a $[\mathrm{Fe} / \mathrm{H}]=-0,27$, abundancia de hierro que resulta representativa del valor medio de las galaxias de la $\mathrm{RCM}$ (ver valores de metalicidad a $z=0$ en el panel inferior de la figura 4.3.)

A modo de ejemplo, calculamos la proporción de masa estelar encerrada en estrellas que viven un tiempo menor o igual al tiempo de Hubble. De la tabla 5.1, se desprende que una estrella de masa $0,9 M_{\odot}$ vivirá un tiempo equivalente a la edad del Universo (13,4694 Gyr según datos de WMAP (Dunkley et al., 2009)). Utilizando la ecuación 5.4, calculamos la fracción de masa cuyos tiempos de vida resultan menores a la edad del Universo; tomamos el valor $0,9 M_{\odot}$ como límite inferior en nuestros cálculos, pues cuanto mayor es la masa de las estrellas más rápida es su evolución, resultando

$$
\int_{0,9 M_{\odot}}^{100 M_{\odot}} 0,171636 m^{-1,35} d m=0,4109 .
$$




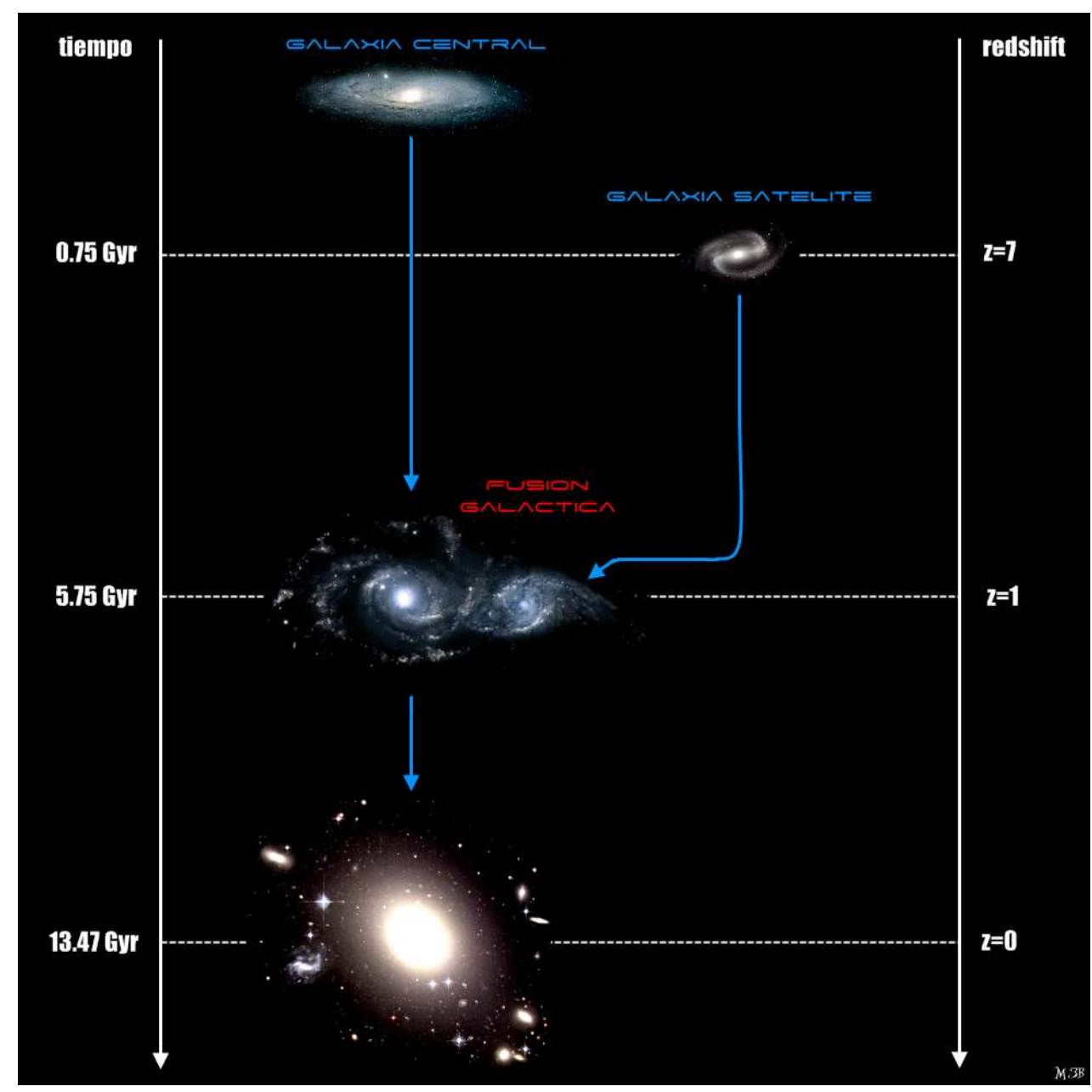

Figura 5.1: Esquema de una situación hipotética en la que una galaxia satélite nacida en $z=7$, que evoluciona aisladamente y se fusiona con una galaxia central en $z=1$. La galaxia remanente evoluciona hasta nuestros días. En este escenario cabe preguntarse qué proporción de la masa original de la galaxia satélite está viva a $z=0$ en la galaxia remanente, y cuánta es la proporción de masa estelar presente a $z=0$ originada debido a la fusión galáctica.

Luego, el 41,09 por ciento de la masa de estrellas formada en un brote estelar, según la ley de Salpeter, tiene un tiempo de vida menor que la edad del Universo, mientras que el 58,91 por ciento restante se aloja en estrellas de masas pequeñas caracterizadas por tiempos de vida mayores que la edad del Universo.

Consideremos ahora un escenario típico de la evolución de las galaxias. Supongamos que en una galaxia satélite se forman, a un dado redshift, todas las estrellas con la misma metalicidad y al mismo tiempo (sea por ejemplo, $z=7$ ). La distribución del número de estrellas nacidas en determinado rango de masas sigue la Ley de Salpeter. Supongamos también que la galaxia formada en $z=7$, continúa su evolución de manera aislada y luego se fusiona con una galaxia central en $z_{\text {merger }}=1$. Representamos esquemáticamente la situación en la figura 5.1. En este escenario, es natural preguntarse qué porcentaje de la

masa estelar formada en la galaxia satélite formada a $z=7$ está viva al momento de la fusión con la galaxia central $(z=1)$ y, de ese porcentaje, cuánto sobrevive hasta $z=0$ 
Cuadro 5.1: Resultados de modelos evolutivos canónicos para estrellas de baja masa, obtenidos de las tablas de BASTI (Pietriferni et al., 2004).

\begin{tabular}{c|c|c}
$Z=0,008 \Leftrightarrow[\mathrm{Fe} / \mathrm{H}]=-0,27$ & Masa estelar $\left(M_{\odot}\right)$ & Tiempo de vida $(\mathrm{Gyr})$ \\
\hline & 0,9 & 13,604 \\
& 1,0 & 9,210 \\
& 1,1 & 6,501 \\
& 1,2 & 4,767
\end{tabular}

y es responsable de los colores que observamos en las galaxias. Por otro lado, si durante la fusión galáctica se forman estrellas, es importante saber cuál es la proporción de esta masa estelar que sobrevive al día de hoy.

Para responder a la primera pregunta, calculamos el intervalo de tiempo transcurrido entre $z=7$ y $z=1$. En el modelo cosmológico $\Lambda \mathrm{CMD}$, con los parámetros usuales descriptos en el capítulo $2, z=7$ corresponde a $t=5,7527$ Gyr, y $z=1$ a $t=0,751$ Gyr. La diferencia de tiempo entre ambos redshifts resulta

$$
\Delta t=5,7527-0,751=5,0017 \mathrm{Gyr} .
$$

En la tabla 5.1 puede verse que una estrella de masa comprendida en el rango $1,1-1,2 M_{\odot}$ tendrá un tiempo de vida comparable al dado por $\Delta \mathrm{t}$. Interpolando linealmente entre estos valores obtenemos una masa límite $M=1,1866 M_{\odot}$. Entonces, todas las estrellas de masas mayores a ésta estarán muertas al momento de la fusión. Para saber qué porcentaje de la masa original creada en $z=7$ representan estas estrellas muertas, integramos la ecuación 5.4 con límites de integración dados por la masa límite encontrada y el valor máximo de la distribución, obteniendo

$$
\int_{1,1866 M_{\odot}}^{100 M_{\odot}} 0,171636 m^{-1,35} d m=0,3640 .
$$

Resulta, entonces, que si una galaxia satélite forma toda su componente estelar en épocas tan tempranas como $z=7$ y luego se fusiona con otra galaxia en $z_{\text {merger }}=1$, la galaxia satélite ya habrá perdido, al momento en que ocurre la fusión, un 36,40 por ciento de su masa estelar original, reteniendo sólo a las estrellas de menor masa que evolucionan más lentamente. Estas estrellas que sobreviven hasta el momento de la fusión representan una fracción de masa de 63,60 por ciento de la masa original, lo cual podemos expresar como

$$
M_{\mathrm{sat}_{\mathrm{z}=1}}=0,636 M_{0}
$$

donde $M_{0}$ es la masa estelar originalmente "nacida" en la galaxia satélite a $z=7$.

Para calcular cuánta de esta masa estelar forma parte de las galaxias a $z=0$, que denotamos como $M_{\mathrm{sat}_{\mathrm{z}=0}}$, estimamos primero la fracción de masa estelar generada en $z=7$ que sobrevive hasta $z=0$. Para ello, tenemos en cuenta que el intervalo de tiempo considerado $(z=7$ a $z=0)$ es $\Delta t=12,7184 \mathrm{Gyr}$, e interpolando entre los valores de la tabla 5.1, obtenemos una masa límite de $M=0,92 M_{\odot}$. Integrando la ecuación 5.5 entre la masa inferior límite encontrada y $100 M_{\odot}$, obtenemos

$$
M_{\mathrm{sat}_{\mathrm{z}=0}}=0,5929 M_{0} .
$$


Combinando este resultado con la ecuación 5.8 se obtiene la fracción de la masa estelar presente en la galaxia satélite al momento de la fusión que forma parte de la galaxia remanente a $z=0$, esto es

$$
M_{\mathrm{sat}_{\mathrm{z}=0}}=(0,5929 / 0,636) M_{\mathrm{sat}_{\mathrm{z}=1}}=0,932 M_{\mathrm{sat}_{\mathrm{z}=1}} .
$$

Por otro lado, en el momento de la fusión, $z=1$, puede tener lugar un brote de formación estelar. Para conocer la fracción de la masa estelar formada, denotada como $M_{\text {star }_{\mathrm{z}=1}}$, que permanece presente a $z=0$, consideramos el tiempo transcurrido entre $z=1$ y $z=0$, esto es

$$
\Delta t=7,7167 \mathrm{Gyr} .
$$

De la interpolación de los valores de la tabla 5.1 correspondientes a tiempos de vida similares al intervalo considerado obtenemos una masa estelar límite de $M=1,055 M_{\odot}$. Luego, calculamos la integral de la expresión 5.5 tomando el valor obtenido como límite inferior, y obtenemos como resultado la masa estelar que forma parte de la galaxia en el presente

$$
M_{\text {stars }_{\mathrm{z}=0}}=0,616 M_{\text {star }_{\mathrm{z}}=1} .
$$

Es decir, la masa formada en el brote estelar ocurrido en $z=1$ se ve reducida en $\approx 38,4$ por ciento como resultado de la evolución de estrellas pertenecientes al extremo masivo de la función inicial de masa de Salpeter.

Estos resultados sirven de guía al momento de interpretar los valores de masa estelar acumulada desde $z=1$ hasta el presente que serán discutidos en la siguiente sección. Como ya hemos mencionado, las variables "sat" y "stars" incluidas en SAGño tienen en cuenta la disminución de la masa estelar que resulta del proceso evolutivo de las estrellas. Este hecho no afecta demasiado a las cantidades acumuladas en el intervalo $0<z<1$ correspondientes a la componente "sat", que registra la masa estelar perteneciente a sistemas satélites que es acretada durante las fusiones, debido a que las poblaciones estelares de los satélites ya han sufrido del proceso de reciclado para la época en que los mismos son acretados; esta afirmación resulta válida si se supone que la mayor parte de la población estelar se formó a $z>1$, según demuestran las estimaciones de fracciones de masa presentes en $z=0$ discutidas anteriormente. Por cierto, si una galaxia satélite acretada durante el intervalo de tiempo $0<z_{\text {merger }}<1$ es creada en épocas tan tempranas como $z=7$, entonces, para el momento en que la misma se fusiona con la galaxia central habrá perdido $\approx 36$ por ciento de su masa estelar original, reteniendo sólo las estrellas de baja masa que evolucionan muy lentamente. Por lo tanto, el $\approx 93$ por ciento de la masa estelar agregada en la fusión ocurrida en $z=1$ continuará viva al día de hoy $(z=0)$. Aún para el caso de una galaxia satélite con componente estelar relativamente jóven formada en $z=2$, que se fusiona con la galaxia central en $0<z_{\text {merger }}<1$, encontramos que más del 90 por ciento de la masa estelar asimilada por la galaxia central, seguirá viva en $z=0$. En cambio, la estimación de la masa de nuevas estrellas formadas acumuladas desde un dado redshift es considerablemente sobreestimada al usar las componentes "stars", ya que las mismas no tienen en cuenta el reciclado de material, que puede llegar a ser del orden de $\approx 38$ por ciento para estrellas formadas en $z=1$. 


\subsection{Agregación de masa desde $z=1$}

Luego de analizar las limitaciones que presentan las variables "stars" y "sat" en cuanto al seguimiento adecuado de la masa estelar generada y acretada, respectivamente, hacemos uso de las mismas para calcular la masa acumulada en el rango de redshifts $0<z<1$, contribuida por ambas componentes. La contribución relativa de estas dos componentes se muestra en la figura 5.2 para las galaxias en los cúmulos C15 en diferentes intervalos de magnitud. Estas fracciones de masas están normalizadas con la masa estelar total promedio de las galaxias a $z=0$ dentro de un dado intervalo de magnitud. Esta elección se debe a que estamos interesados en la importancia relativa de la masa acumulada desde $z=1$ respecto de la masa estelar total de la galaxia en el presente. Nótese que esta última es la masa estelar resultante de la evolución completa trazada por el código semianalítico, incluyendo su reducción debido al reciclado de masa inherente al proceso de evolución estelar. Como puede verse en la figura, la fracción de masa estelar correspondiente a la componente "stars" posee valores que van desde $\approx 0,05$ para las galaxias más luminosas, $\mathrm{a} \approx 0,15$ para las galaxias más débiles. Sin embargo, estos valores son menores si se tiene en cuenta que las componentes estelares se normalizan respecto de la masa total de la galaxia a $z=0$, la cual ha perdido 38,4 por ciento de la masa, como consecuencia de la evolución estelar de las estrellas pertenecientes al extremo masivo de la función inicial de masas de Salpeter. La componente "stars" no tiene en cuenta esta reducción de masa, tal como se discutió al final de la sección anterior. Las fracciones de masa estelar contribuidas por la masa estelar acretada durante las fusiones de galaxias, tenidas en cuenta por la componente "sat", no son tan afectadas por la definición de las cantidades acumuladas debido a que la población estelar de las galaxias satélites ya han sufrido el proceso de reciclado para el momento en que son acretadas, suponiendo que la mayor parte de sus estrellas se formaron en $z>1$. De este modo, más de un 90 por ciento de la masa estelar agregada durante las fusiones permanece viva en $z=0$, tal como se discutió en la sección anterior. Teniendo en cuenta estas consideraciones, podemos concluir del análisis de la figura 5.2 que $\approx 10-20$ por ciento de la masa de las galaxias en el extremo brillante de la RCM proviene de la acreción de galaxias satélites desde $z=1$ (componente "sat"), con muy pocas estrellas formadas in situ en ése período (componente "stars").

Un aspecto interesante a resaltar de la figura 5.2 es que los aportes de las componentes "stars" y "sat" se cruzan en la magnitud de quiebre de la RCM, $M_{T_{1}}=-20$, indicando claramente que la población estelar agregada durante las fusiones tardías determina las propiedades de las galaxias en el extremo brillante de la RCM. Esta magnitud de quiebre en la cual cambia apreciablemente la pendiente de la RCM, corresponde también a galaxias con masa de $M_{\star} \sim 10^{10} h^{-1} \mathrm{M}_{\odot}$. Es notable la coincidencia de estos valores con los resultados observacionales que reporta Baldry et al. (2006), tanto para el quiebre de la RCM, como para la masa característica en la que el mismo sucede. Esta masa también marca la diferencia entre dos regímenes en la dependencia de los gradientes de metalicidad con la masa de las galaxias, encontrado por Spolaor et al. (2009). Sus resultados sugieren que las galaxias de cúmulos de masas mayores que este umbral podrían haberse formado inicialmente por fusiones de galaxias de disco ricas en gas y luego haber evolucionado mediante eventos de fusión seca, en acuerdo con nuestras conclusiones.

\subsection{Comparación con resultados observacionales}

\subsubsection{Fracciones de masa formada in situ}

Las fracciones de masa que han sido formadas in situ desde $z=1$, que tienen en cuenta todos los procesos físicos que contribuyen con gas frío a partir del cual se originan las nuevas estrellas, pueden ser comparadas directamente con las estimaciones de Kaviraj et al. (2008). Estos autores calculan la formación estelar reciente (FER; definida como la 
fracción de masa estelar formada en la galaxia en el último Gyr de look-back time en el marco de referencia en reposo) en la población de galaxias tipo temprano,a alto redshift $(0,5>z>0,1)$, basándose en los colores UV y ópticos en el marco de referencia en reposo y combinando estos resultados con estudios similares en el universo local (Kaviraj et al., 2007). Este análisis les permite estimar la historia de formación estelar de la población de ETG entre $z=0$ y $z=1$. Para el rango $1>z>0,5$, las ETGs de la secuencia roja utilizada en su estudio $(N U V-r>4)$ muestran valores de FER menores al 5 por ciento. Más aun, las ETGs más rojas, que son también las más luminosas, son prácticamente inactivas con una FER de apenas un $\sim 1$ por ciento.

Por otro lado, Kaviraj et al. (2007) encuentran que la actividad de formación estelar en las ETGs más activas (aquéllas que forman el pico azul en las distribuciones de color magnitud), se ha reducido a la mitad desde $z=0,7$ a $z=0$, pasando de un valor promedio de FER de $\sim 11$ por ciento, en $z=0,7$, al valor actual de sólo $\sim 6$ por ciento. De estos resultados los autores infieren que $\sim 10-15$ por ciento de la masa estelar en las ETGs luminosas $\left(-24<M_{V}<-20,5\right)$ pudo haberse formado despues de $z=1$, mientras que las galaxias menos luminosas pueden haber formado $\sim 30-60$ por ciento de su masa en el mismo intervalo de tiempo.

Las fracciones de masa estelar formadas in situ (componente "stars") desde $z=1$ dadas por nuestro modelo, mostradas por la línea a rayas en la figura 5.2, son aproximadamente un factor 2 más bajas que los resultados observacionales de Kaviraj et al. (2007), con valores en los rangos $\sim 0,05-0,09$ por ciento y $\sim 0,09-0,15$ por ciento para galaxias en los extremos brillante y débil de la RCM, respectivamente. Sin embargo, esta diferencia es esperable si se tiene en cuenta que el estudio de (Kaviraj et al., 2008) abarca galaxias en una variedad de entornos muy diferentes, mientras que nuestras simulaciones corresponden a cúmulos de galaxias, estructuras caracterizadas por una evolución más rápida que los grupos de galaxias y las distribuciones de galaxias de campo, en los cuales el pico de la tasa de formación estelar tiene lugar en momentos más tempranos que lo acontecido para galaxias en medios de menor densidad. En consecuencia, consideramos que nuestros resultados se encuentran en muy buen acuerdo con las inferencias observacionales.

\subsubsection{Tasas de fusiones}

La cuantificación observacional del impacto de las fusiones galácticas en el ensamblado de las ETGs arroja mayores incrementos en la masa de las galaxias desde $z=1$, respecto de los valores de fracciones de masa estelar acumulada contribuidos por la componente "sat" que hemos encontrado, dados por la línea punteada en el panel superior de la figura 5.2. Sin embargo, debe tenerse en mente que nuestros resultados corresponden a galaxias en cúmulos, caracterizados por una rápida evolución, como ya se mencionó. En particular, Lopez-Sanjuan et at. (2010) encuentran que las ETGs más brillantes aumentan su masa alrededor de $42 \pm 8$ por ciento, como consecuencia de las fusiones galácticas sufridas desde $z=1$ al día de hoy. En este estudio los autores utilizan pares cercanos confirmados cinemáticamente encontrados en el relevamiento de espectroscopía profunda realizado con el Visible MultiObject Spectrograph del Very Large Telescope (VIMOS-VLT), para estudiar el rol de las fusiones menores en el ensamblado de galaxias normales $L_{B} \gtrsim L_{B}^{*}$. Es importante notar que en este estudio las galaxias pueden pertenecer a diferentes ambientes (campo, grupos o cúmulos). El hecho de que la fracción de masa recibida por las ETGs masivas en la muestra de Lopez-Sanjuan et at. (2010) sea un factor 2 más grande que el encontrado para las galaxias más brillantes en nuestras simulaciones es una consecuencia del menor número de fusiones que sufren las galaxias pertencientes a cúmulos desde $z=1$.

La estimación del número promedio de fusiones por cada galaxia roja desde $z=1$ realizada por Lopez-Sanjuan et at. (2010) es 1,3 $\pm 0,3$, discriminado en valores de 0,7 $\pm 0,1$ para fusiones mayores y de $0,6 \pm 0,2$ para fusiones menores. El criterio utilizado por estos 

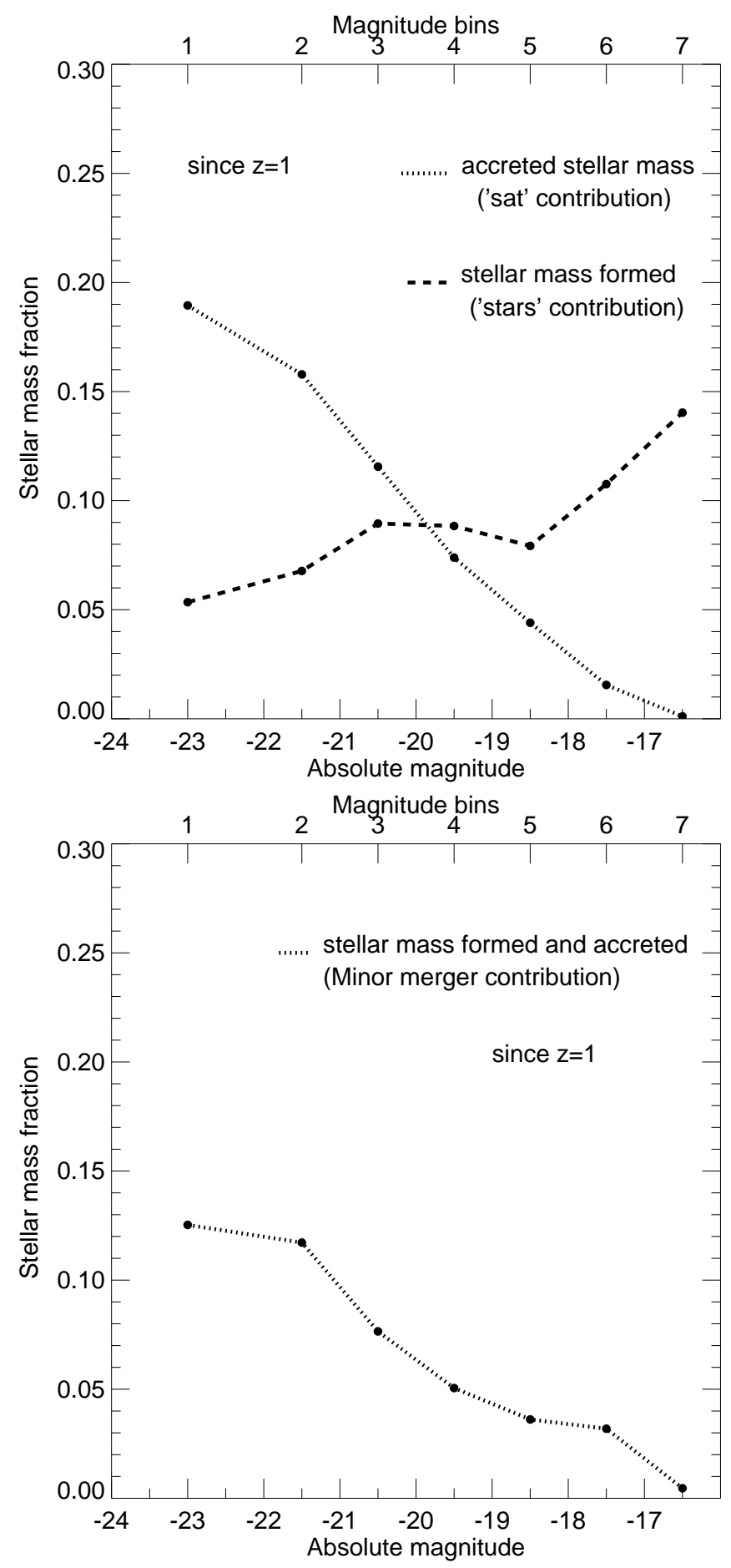

Figura 5.2: Panel superior: Fracciones de masa estelar contribuidas por las componentes "stars" (línea a rayas) y "sat" (línea punteada), acumuladas desde $z=1$, para cada intervalo de magnitud. Nótese como las fracciones de masa contribuidas por los procesos "sat" y "stars" se cruzan en la magnitud crítica correspondiente al quiebre de la RCM $\left(M_{T 1} \sim-20\right)$. Panel inferior: Fracciones de masa estelar contribuida por la componente "sat" para cada intervalo de magnitud, acumulada desde $z=1$, debida a fusiones menores únicamente. En ambos casos, las fracciones de masa han sido normalizadas con la masa estelar total de las galaxias en $z=0$ en cada intervalo de magnitud; se consideran las galaxias en los cúmulos C15. 
autores para discriminar entre fusiones galácticas mayores y menores está basado en el cociente de luminosidades en la banda $B$ de las galaxias que se fusionan; se supone que ésta luminosidad es un buen representante de la masa de las galaxias. Específicamente, clasifican como fusiones menores a aquéllas caracterizadas por cocientes de luminosidades en el rango de 0,1 a 0,25. En nuestro modelo, las fusiones menores se definen como aquéllas que poseen cocientes de masa bariónica menores a 0,3, sin la imposición de ningún tipo de límite inferior. A partir de nuestro modelo hallamos que las galaxias del extremo brillante de la $\operatorname{RCM}\left(M_{T 1} \leq-20\right)$ han sufrido un número promedio de fusiones de $\sim 4,88$ desde $z=1$. Este número es aproximadamente un factor 4 más grande que el encontrado por Lopez-Sanjuan et at. (2010), posiblemente debido a la falta de consideración de un límite inferior en el cociente que interviene en nuestra definición de fusiones menores. Por lo tanto, hemos repetido la estimación del número de fusiones restringiendo el conteo de fusiones menores a aquéllas caracterizadas por cocientes de masa mayores a 0,1, de acuerdo con el límite inferior tomado por Lopez-Sanjuan et at. (2010). En ese caso, encontramos que el número de fusiones promedio sufridas por galaxias en el extremo brillante se reduce considerablemente a un valor de $\sim 0,33$ fusiones menores por galaxia. Este valor es un factor 2 menor que el inferido por Lopez-Sanjuan et at. (2010), pero debemos tener en cuenta que, como dijimos antes, nuestra muestra está restringida a cúmulos de galaxias donde la evolución ocurre más rápidamente que en otros ambientes.

La fracción de masa estelar acumulada aportada por las fusiones menores desde $z=1$ obtenida a partir de nuestro modelo se muestra en el panel inferior de la figura 5.2. Esta fracción alcanza valores de $\sim 0,12$ y $\sim 0,07$ para las galaxias más y menos luminosas del extremo brillante de la RCM, respectivamente. Estos valores están en muy buen acuerdo con el valor $\sim 0,1$ estimado por Lopez-Sanjuan et at. (2010). Los valores que se muestran en el panel inferior de la figura 5.2 se han obtenido de la misma manera que aquéllos mostrados en el panel superior de dicha figura, pero considerando solamente la masa contribuida por la componente "sat" de las fusiones menores, las cuales incluyen aquellas fusiones con cocientes de masas bariónicas menores a 0,1. Esto indica que las fusiones menores caracterizadas por tales cocientes de masas, si bien son muy numerosas, agregan un porcentaje de masa muy pequeño a la masa estelar de la galaxia central.

Por otro lado, el número promedio de fusiones mayores sufrido por las galaxias más brillantes en la RCM desde $z=1$ es de $\sim 0,19$. Este valor es un factor 3 más pequeño que el valor 0,7 $\mp$ 0,1 obtenido por Lopez-Sanjuan et at. (2010), dando lugar al hecho de que la fracción de masa aportada por todos los tipos de fusiones a las galaxias más brillantes de la RCM ( 0,2; ver panel superior de la figura 5.2) sea un factor 2 más pequeño que el valor $\sim 0,42$ observado por Lopez-Sanjuan et at. (2010). Sin embargo, el valor del número de fusiones mayores inferido a partir de nuestro modelo está en mejor acuerdo con otras determinaciones empíricas de tasas de fusiones mayores, lo cual indica que la actividad de esta clase de fusiones en galaxias con masas comparables a las que resultan en nuestro modelo se vuelven menos frecuentes desde $z=1$ (v.g., Conselice et al. 2009; Bundy et al. 2009; Jogee et al. 2009).

Así mismo, comparamos nuestros resultados con aquéllos obtenidos por Nierenberg et al. (2011). Estos autores estudian la distribución espacial de galaxias satélites poco luminosas de galaxias centrales de tipo temprano a redshifts intermedios $(0,1<z<0,8)$. Seleccionan la muestra de galaxias del campo de GOODS, Great Observatories Origins Deep survey. Las galaxias satélites son detectadas en imágenes del Hubble space Telescope de alta resolución, considerando aquéllas que son hasta 5,5 veces más débiles que la galaxia anfitriona y que se encuentran a una distancia mínima de hasta $0^{\prime \prime} 5 / 2,5 \mathrm{Kpc}$ de la misma. La mayoría de las galaxias anfitrionas de ese estudio son galaxias elípticas masivas con masas estelares típicas de $\approx 10^{10,5} M_{\odot}$ que poseen, en promedio, $1,7_{-0,8}^{+0,9}$ satélites. Este número inferido de galaxias satélites puede dar cuenta fácilmente del número de fusiones menores $(0,4-0,5)$ que se espera que sufran el 60 por ciento de sus galaxias anfitrionas en el intervalo de tiempo abarcado por su estudio. Este número de fusiones ha sido obtenido utilizando la expresión del ajuste para la tasa promedio de fusiones por halo 


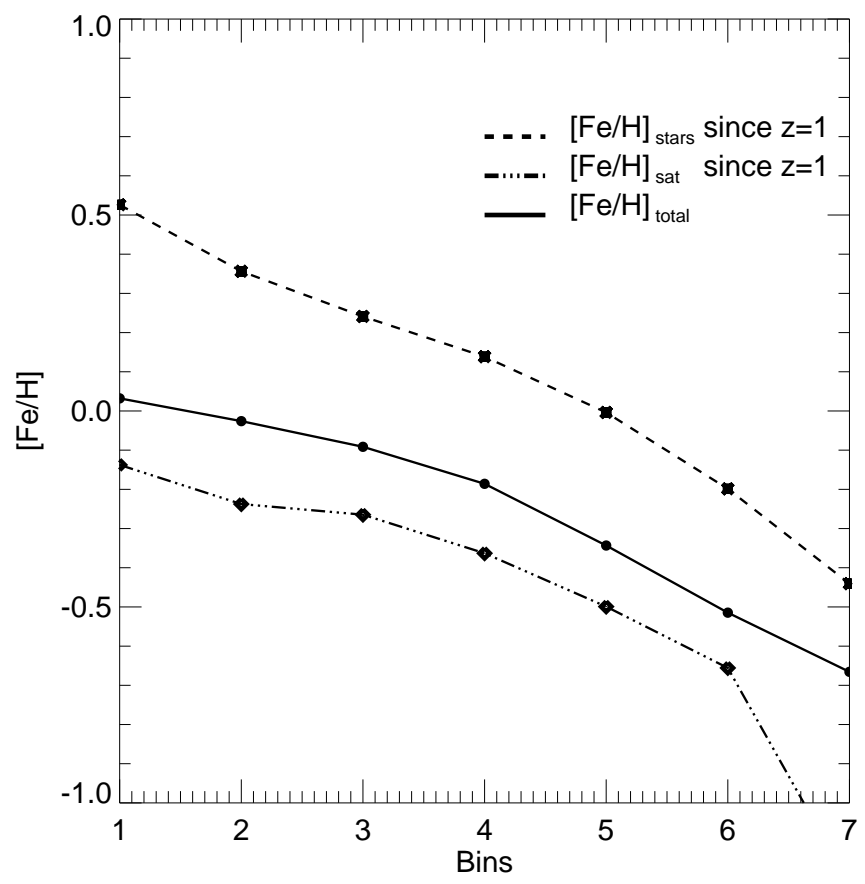

Figura 5.3: Abundancia media de hierro de las galaxias en $z=0$ tal como surge de la evolución completa computada en el modelo semianalítico (línea continua), para galaxias en los diferentes intervalos de magnitud. Estos valores aparecen comparados con la metalicidad del material aportado por las estrellas formadas desde $z=1$ (línea de trazos) y con la de aquellas estrellas que ya están presentes en las galaxias satélites que fueron acretadas desde $z=1$ (línea de punto y raya).

como función de la masa del halo, del cociente de masas del progenitor y del redshift, derivada por Fakhouri et al. (2010) a partir de estadísticas de fusiones de halos. Si bien los resultados de nuestro modelo ( $\sim 0,33$ fusiones menores por galaxia desde $z=1$ ) son un poco menores que estos valores inferidos, los mismos resultan consistentes con estos últimos.

\subsection{Contribución de metales desde $z=1$}

A partir del análisis de la figura 5.2 detallado en la sección concluimos que la población estelar acretada durante las fusiones ocurridas en $z<1$ juega un papel dominante en la determinación de las propiedades de las galaxias en el extremo brillante de la RCM. Podemos suponer con seguridad que más del 93 por ciento de la masa estelar agregada y más del 61 por ciento de la masa formada in situ desde $z=1$ está aún viva en $z=0$, influyendo en la metalicidad promedio y el color de las galaxias de la RCM al día de hoy. A fin de evaluar el impacto de las componentes "stars" y "sat" en estas propiedades, estimamos la metalicidad de la masa estelar aportada por cada una de estas dos componentes, a las que denotamos como $[\mathrm{Fe} / \mathrm{H}]_{\text {stars }}$ y $[\mathrm{Fe} / \mathrm{H}]_{\text {sat }}$. La estimación se realiza mediante el cómputo de la masa de hierro, $\mathrm{Fe}_{g, z}^{\mathrm{proc} \text { comp }}$, y la masa de hidrógeno, $\mathrm{H}_{g, z}^{\text {proc comp }}$, que fueron definidas en la sección 4.2 del capítulo anterior. El valor promedio de estas metalicidades para las galaxias en los cúmulos $\mathrm{C} 15$ en diferentes intervalos de magnitud se presenta en la figura 5.3. Estas cantidades se comparan con la metalicidad 
promedio alcanzada por la masa estelar de las galaxias a $z=0$ debida a todos los procesos evolutivos considerados en SAG (línea continua), la cual yace en el rango de $-0,65 \lesssim[\mathrm{Fe} / \mathrm{H}]_{\text {total }} \lesssim 0,05$, cuyos extremos inferior y superior corresponden a las galaxias menos y más luminosas de la RCM, respectivamente.

Nótese que el rango de metalicidades de las estrellas formadas desde $z=1$ (línea de trazos) se extiende desde $[\mathrm{Fe} / \mathrm{H}]_{\text {star }} \approx-0,45$ para las galaxias menos luminosas, hasta $\approx 0,5$ para las más luminosas. En particular, para las galaxias en el extremo brillante de la RCM, con magnitudes entre $-24 \leq M_{\mathrm{T}_{1}} \leq-20$ (i.e. intervalos 1,2 y 3 ) la metalicidad de la componente "stars" está por encima de la metalicidad solar $\left(0,25 \lesssim[\mathrm{Fe} / \mathrm{H}]_{\text {stars }} \lesssim 0,5\right)$. Esto es consecuencia de la alta metalicidad del gas frío disponible para la formación estelar en las galaxias más masivas. Sin embargo, como hemos visto, la fracción de masa aportada por los procesos que involucran formación estelar es muy baja para estas galaxias (ver figura 5.2), y por lo tanto esta contribución de alta metalicidad difícilmente altere la metalicidad promedio de toda la galaxia. Por otro lado, la componente "sat" de los satélites acretados desde $z=1$ representa una fracción apreciable de la masa de la galaxia a $z=0$ para aquellas galaxias correspondientes al extremo más brillante $(\approx 10-20$ por ciento), pero los valores de $[\mathrm{Fe} / \mathrm{H}]_{\text {sat }}$ (línea de punto y raya en el panel inferior de la figura 5.3), son subsolares para las galaxias en todos los intervalos de magnitud, siendo $\approx 0,15$ dex menores que la correspondiente metalicidad final de las galaxias.

En consecuencia, podemos concluir que las fusiones secas ayudan a aumentar la masa de las galaxias en el extremo brillante de la RCM, sin afectar considerablemente sus metalicidades promedio. El límite superior en la metalicidad alcanzada por las galaxias en el extremo brillante de la RCM fija sus colores, resultando más azules de lo que sería esperable si fuese relevante la formación estelar a partir de un gas altamente enriquecido en metales en la fase evolutiva final de estas galaxias. Esto se traduce en un apartamiento del extremo brillante de la RCM respecto del ajuste lineal usualmente usado para toda la relación. Explicamos asi la razón del quiebre de la RCM, que motiva esta investigación.

\subsection{Discusión de los resultados: Influencia del ambiente}

Parte de los resultados presentados a lo largo de este capítulo muestran que las galaxias satélites acretadas a bajos redshifts $(z<1)$ están formadas por poblaciones estelares caracterizadas por bajos valores de metalicidad con respecto a las abundancias químicas encontradas en las galaxias remanentes. Este hecho está en acuerdo con los resultados de Lagos et al. (2009), quienes muestran una clara correlación entre la metalicidad de las galaxias y la masa de los halos de materia oscura que éstas habitan, siendo las galaxias centrales más enriquecidas químicamente que los satélites sobrevivientes. Esta tendencia es un efecto directo del proceso de crecimiento jerárquico de estructuras, donde las galaxias más masivas se situan en picos de inicialmente más alta densidad y, en consecuencia, comienzan su formación en épocas más tempranas, en promedio. Por lo tanto, las galaxias más masivas alcanzan un contenido de metales mayor que las menos masivas.

Nuestros resultados concuerdan con el escenario propuesto por Martínez et al. (2010) para la formación de la RCM en sistemas de alta masa. Su análisis de resultados observacionales indica que la RCM está poblada, por un lado, por ETGs rojas que formaron el grueso de sus estrellas durante las etapas tempranas del ensamblado de los halos masivos y, por otro lado, por galaxias rojas que pasaron la mayor parte de sus vidas en ambientes de baja densidad de galaxias, como son los grupos o el campo, y caen luego dentro de los sistemas masivos, a bajos redshifts. Nuestro estudio sugiere que parte de esta última población se identifica con las galaxias satélites que intervienen en las fusiones galácticas secas, las cuales juegan un papel importante en la evolución tardía de las galaxias masivas.

El hecho de que las galaxias masivas de los cúmulos sean alimentadas por fusiones secas, mientras que galaxias menos luminosas de la RCM sean principalmente afectadas 
por las fusiones menores húmedas, es debido a la influencia del entorno en el cual residen las galaxias satélites y las anfitrionas. Galaxias más masivas tienden a habitar regiones más densas donde es altamente probable que las satélites acretadas sean desprovistas de su gas antes de fusionarse con las galaxias centrales como consecuencia de efectos ambientales. Por lo tanto, el modelado de los procesos ambientales que modifica el contenido del gas frío de las galaxias puede afectar nuestras conclusiones respecto de la fracción de masa recibida por las galaxias en fusiones húmedas y secas. Así mismo, pueden verse afectadas también las conclusiones sobre el número de fusiones galácticas de distinto tipo.

No hay consenso general sobre la tasa de fusiones predicha por los modelos semianalíticos y las simulaciones hidrodinámicas autoconsistentes. Dependiendo del método usado en los modelos para convertir la historia de crecimiento de los halos de materia oscura en la historia de crecimiento de las galaxias se encuentran diferencias en las predicciones de la tasa de fusión, aún cuando se usa el mismo modelo cosmológico. Hopkins et al. (2010) estudia la dependencia de las tasas de fusión de las galaxias en función de la tasa de fusión de los halos de materia oscura, y de la manera en que las galaxias pueblan estos halos, en diferentes modelos de formación de galaxias. Los autores concluyen que la tasa de fusión galáctica depende fuertemente de éste último factor, es decir, de la manera en que las galaxias pueblan los halos de materia oscura. De su análisis concluyen que la mayoría de los modelos predice para galaxias luminosas, un número similar en la tasa de fusiones galácticas y todos los modelos concuerdan en que el proceso primario de formación de bulbos galácticos es el de la fusión mayor entre galaxias (Hopkins et al., 2010; Parry et al., 2009; Cattaneo et al., 2011).

En nuestro modelado, como en la mayoría de los códigos semianalíticos, los halos de gas caliente de las galaxias son removidos de manera completa e inmediata tan pronto como las mismas se transforman en galaxias satélite de un cúmulo o grupo. La galaxia afectada no puede luego reponer la reserva de gas frío, ya que sólo las galaxias centrales reciben cooling flows, lo que causa el agotamiento de su gas frío disponible en unos pocos Gyr (manteniendo una tasa típica de formación estelar, Gallagher et al. 1989). Este proceso, conocido como estrangulación (Larson et al., 1980), proporciona una explicación para los gradientes en las tasas de formación estelar de los cúmulos de galaxias (Kodama \& Bower, 2001). Sin embargo, trabajos recientes (Weinmann et al., 2006, v.g) han mostrado que, comparados con las observaciones del SDSS, los modelos semianalíticos producen una fracción excesiva de galaxias rojas debido a que ven truncado su proceso de formación estelar al consumir el gas frío disponible. A este efecto se lo conoce en la literatura con el nombre de "overquenching" (Kimm et al., 2007). Por otro lado, existe evidencia observacional que establece que la fracción de galaxias satélites rojas que han sufrido la remoción del gas es una función dependiente de la masa estelar de las galaxias satélites. Esta fracción muestra una dependencia débil con la densidad local o la masa del halo de materia oscura, tanto a $z=0$ como a alto redshift $(z \sim 1-1,5)$ (Guerke et al., 2007; Haines et al., 2007). Así mismo, se ecuentra evidencia observacional de una dependencia débil de la fracción de satélites rojas con la distancia al centro del halo Weinmann et al. (2006).

El overquenching de las galaxias satélites es muy probablemente debido al modelado altamente simplificado de la remoción de gas caliente incluido en los modelos semianalíticos. Si el gas caliente fuese removido de manera más gradual, las galaxias satélites prolongarían su formación estelar, lo que resultaría en colores integrados más azules. Si bien los procesos físicos responsables de la remoción de gas caliente no son conocidos con certeza, los candidatos más probables son la "ram-pressure stripping" (RPS), combinada con la "tidal stripping", tal como ha sido mostrado por McCarthy et al. (2008), Bekki (2009) y Kimm et al. (2011).

El problema del overquenching de las galaxias satélites trae a colación el hecho de que quizás los modelos semianalíticos sobreestiman el número de fusiones galácticas menores secas, ya que las galaxias satélites del modelo resultarían demasiado empobrecidas en gas. Con respecto a esto, el trabajo de Font et al. (2008) muestra que al considerar una remoción gradual del gas caliente por RPS se obtiene un mejor acuerdo con las fracciones 
de galaxias rojas observadas. Uno debería concluir que, con una modificación similar en nuestro modelo, el número de fusiones húmedas debería aumentar. Sin embargo, la situación no es tan simple. Si el contenido de gas frío de las galaxias satélites está regulado por la RPS de su gas caliente (que sirve de reserva para dar lugar al aumento de gas frío), entonces el color de las galaxias satélites sería más azul como resultado de una formación estelar más prolongada. Pero esto no significa que una mayor cantidad de gas sea finalmente aportada a las galaxias centrales en las fusiones. En las regiones centrales de los cúmulos de galaxias la RPS ejercida por el medio intracúmulo es suficientemente fuerte como para afectar significativamente el contenido de gas frío de las galaxias (Tecce et al., 2010) y, para el tiempo en que las galaxias satélites se fusionan con las galaxias centrales, no sólo el gas caliente sino también el gas frío han sido removidos como consecuencia de la RPS, transformándose en parte del medio intracúmulo. Por lo tanto, el contenido de gas de las galaxias satélites sería removido casi completamente antes de la fusión (Sofue, 1994; Grebel et al., 2003) y el número de fusiones húmedas no aumentaría significativamente.

El impacto del efecto de la RPS del gas frío en las propiedades de galaxias satélites en cúmulos de galaxias fue investigado en el Trabajo de Tesis de Tomás E. Tecce. En particular, se analiza la influencia de este proceso en las características del diagrama color magnitud, encontrándose que la RPS comienza a ser importante a $z<1$, cuando la secuencia roja en el cúmulo ya está establecida, y que las distribuciones de colores obtenidas a partir de los modelos con y sin RPS son muy similares para las galaxias más masivas (ver figura 5 de su Tesis). Esto se debe a que el proceso de estrangulación actúa en todos los halos y para todo redshift, por lo que las galaxias simuladas ya son relativamente pobres en gas y con baja SFR para cuando son afectadas por RPS. Por lo tanto, nuestras conclusiones sobre los procesos relevantes que conducen al desarrollo del quiebre de la RCM no se verían mayormente afectados por el efecto de la RPS cuando se considera el proceso de estrangulación, que es el caso del modelo semianalítico utilizado en el presente Trabajo de Tesis. A futuro, planeamos explorar el efecto de la RPS en la RCM cuando el gas caliente de las galaxias satélites es removido en forma gradual, considerando una reciente actualización del SAG donde se incluye un nuevo modelo del proceso de RPS del gas caliente (Tecce et al. 2012, en prep.). 


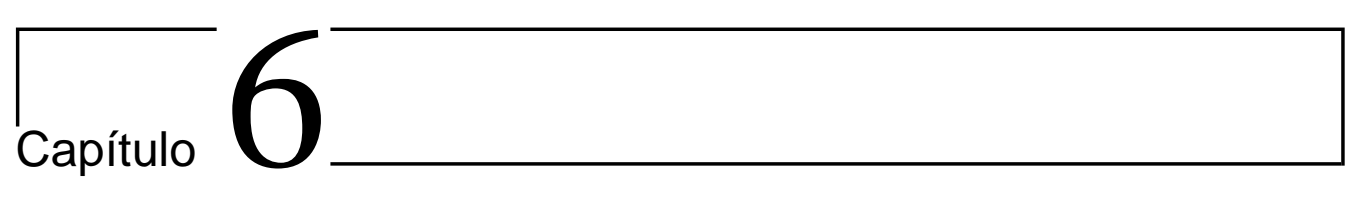

\section{Nuevas implementaciones en SAG: Efectos sobre la RCM}

En el presente capítulo estudiamos el impacto sobre las propiedades de la RCM de nuevas implementaciones incluídas en el modelo semianalítico SAG, que alteran los colores, las edades y las metalicidades de las galaxias que pueblan la RCM. Estas implementaciones incluyen el efecto de la extinción por polvo, la inclusión de la emisión de estrellas en la fase TP-AGB, y una formulación alternativa para describir el proceso de formación estelar.

El nuevo modelo de extinción por polvo que aquí utilizamos es descripto por Guo \& White (2009). Para tener en cuenta el efecto de las estrellas en la fase TP-AGB, adoptamos los modelos evolutivos de síntesis de poblaciones desarrollados por Charlot \& Bruzual (2007). Por último, consideramos el modelo de formación estelar propuesto por Croton et al. (2006). Analizamos la influencia sobre la RCM de cada implementación por separado y el efecto combinado de la acción simultánea de todas ellas, manteniendo siempre los parámetros de calibración de SAG utilizado en todo el análisis previo. Esto se hace a fin de evaluar el impacto de estos cambios en los resultados y conclusiones descriptos en los capítulos anteriores.

\subsection{Nuevo modelo de extinción por polvo}

El código semianalítico SAG aplicado en este trabajo, incluye el modelado de la extinción causada por el polvo interestelar propuesto por Kauffmann et al. (1999), tal como se describe en la sección 2.3.8. Este modelo de extinción es simple y está basado en una correlación empírica entre cantidades macroscópicas de las galaxias, como su luminosidad y la profundidad óptica del ISM. A bajo redshift, el modelo permite obtener un buen acuerdo con distintos observables que involucran la luminosidad y el color de las galaxias, como por ejemplo, la función de luminosidad. Notablemente, el acuerdo es bueno aun en la banda $B$ donde la absorción por polvo es predominante. En adelante, llamamos " $\mathrm{SAG}_{\mathrm{E} 1}$ " a la versión del código semianalítico que usa este modelado de extinción por polvo.

Claramente, la extinción por polvo interestelar debe ser tenida en cuenta en los modelos de galaxias a fin de comparar los resultados con los datos observados. Numerosos intentos por mejorar las formulaciones para la extinción por polvo incluyen modelos basados en aquél propuesto originalmente por Guideroni \& Rocca-Volmerange (1987). Este modelo supone una relación entre la profundidad óptica del polvo a una dada longitud de onda, $\lambda$, y la metalicidad del gas frío. Teniendo en cuenta la gran cantidad de datos actualmente disponibles de relevamientos profundos es importante contar con una ley de 
extinción que dependa también del redshift. Esta necesidad surge de la evidencia observacional de que galaxias con la misma luminosidad bolométrica y metalicidad que a bajo redshift, presentan un cociente entre polvo y gas menor a alto redshift (Quadri et al., 2008). La inclusión de la dependencia con el redshift en los modelos de extinción permite reproducir adecuadamente la abundancia y el clustering de galaxias a redshifts $z \sim 2$ y $z \sim 3$ (Guo \& White, 2009).

Por lo tanto, de acuerdo a lo propuesto por Guo \& White (2009), implementamos en SAG un nuevo modelo de extinción donde la opacidad óptica media dependende de $\lambda$, la metalicidad del gas frío y el redshift. La forma matemática que adopta para un disco visto de frente es

$$
\tau_{\lambda}^{\mathrm{Z}}=\left(\frac{A_{\lambda}}{A_{V}}\right)_{Z_{\odot}}\left(\frac{Z_{\mathrm{gas}}}{Z_{\odot}}\right)^{s}(1+z)^{-0,4}\left(\frac{\left\langle N_{H}\right\rangle}{2,1 \times 10^{21} \mathrm{~cm}^{-2}}\right)
$$

donde $\left(A_{\lambda} / A_{V}\right)_{Z_{\odot}}$ es la curva de extinción obtenida por Cardelli et al. (1989), $Z_{\text {gas }}$ es

la metalicidad del gas frío, y $\left\langle N_{H}\right\rangle$ es la densidad columnar promedio de hidrógeno dada por

$$
\left\langle N_{H}\right\rangle=\frac{M_{\mathrm{gas}}}{1,4 m_{\mathrm{p}} \pi r^{2}} \mathrm{~cm}^{-2} .
$$

En esta última expresión, la cantidad $m_{\mathrm{p}}$ es la masa del protón, $M_{\text {gas }}$ es la masa de gas frío del disco galáctico y $r$ es el radio exponencial de escala del disco; el factor 1,4 tiene en cuenta la presencia de helio. El exponente al cual se eleva la metalicidad del gas frío, $s$, varía con la longitud de onda. De acuerdo a la interpolación de las curvas de extinción medidas en el vecindario solar y en la Nube Menor y Mayor de Magallanes, el valor del exponente es $s=1,35$ para $\lambda<2000$, y $s=1,6$ para $\lambda>2000$. Finalmente, para calcular la extinción efectiva, se toman inclinaciones al azar para cada disco galáctico y, de acuerdo a la aproximación de una geometría de capas planas paralelas, se aplica la fórmula 2.3.8.

Es importante enfatizar que las cantidades involucradas en la ecuación (6.1) se obtienen del modelo semianalítico SAG para cada galaxia y en cada redshift. La versión del código SAG que incluye esta nueva implementación del modelado de extinción por polvo se llamará $\mathrm{SAG}_{\mathrm{E} 2}$. La principal ventaja de esta nueva versión del código reside en que el modelo de extinción depende de numerosas propiedades que caracterizan a las galaxias, mientras que el modelo implementado en $\mathrm{SAG}_{\mathrm{E} 1}$ sólo vincula la profundidad óptica del ISM con la luminosidad de la galaxia en una determinada banda. En el presente estudio, nos limitamos al análisis de la RCM a $z=0$, por lo que no hacemos uso de la dependencia con el redshift del modelo de extinción, que fue la mejora introducida por Guo \& White (2009). A futuro, la implementación de esta formulación permitirá extender los métodos de análisis aquí aplicados al estudio de las características de la RCM a alto redshift.

\subsubsection{Efectos de la extinción sobre la RCM}

El primer paso para caracterizar las diferencias entre los colores de las galaxias que forman la RCM debidas a los diferentes modelos de extinción utilizados en SAG consiste en extraer totalmente cualquier correción por polvo de las galaxias del modelo y analizar la RCM resultante. Esto se realiza con el sólo fin de evidenciar los efectos de los dos modelos de extinción sobre los colores de las galaxias, pero debemos tener en cuenta que un modelo que no incluya el efecto de extinción por polvo sería incompleto y resultaría incorrecto, ya que no podemos ignorar la existencia ubicua de la componente de polvo en las galaxias. En este sentido, cabe destacar el trabajo de Kauffmann et al. (1999), 

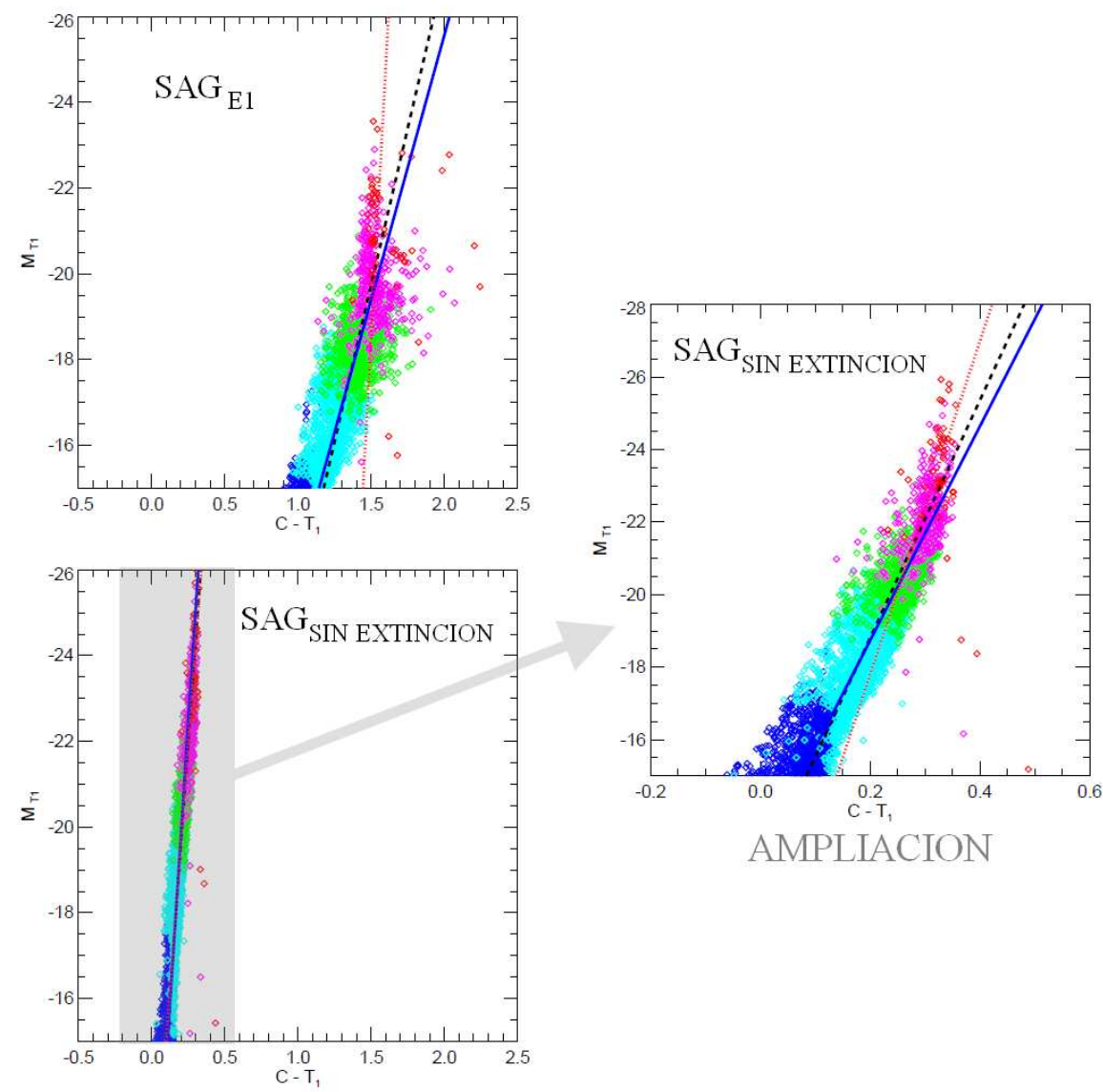

Figura 6.1: Relación Color Magnitud del cúmulo de referencia, $\mathrm{C} 15_{3}$ (g51), en el sistema de Washington, con el efecto de extinción por polvo original adoptada en SAG y sin corrección por polvo. Panel superior izquierdo: La RCM resultante de aplicar la versión original del código, $\mathrm{SAG}_{\mathrm{E} 1}$; las líneas azul llena, roja punteada y negra de trazos, corresponden a ajustes por mínimos cuadrados al extremo débil, al brillante y a toda la relación, respectivamente. Panel inferior izquierdo: La RCM sin efectos de extinción por polvo; las líneas de colores marcan los ajustes por mínimos cuadrados de la misma manera que en el panel superior. Panel medio derecho: Se muestra una ampliación de la misma RCM sin extinción por polvo presentada en el panel inferior izquierdo, con cada una de las rectas de ajustes, en una escala menor en los colores a fin de visualizar la presencia del quiebre en el extremo brillante de la relación. 

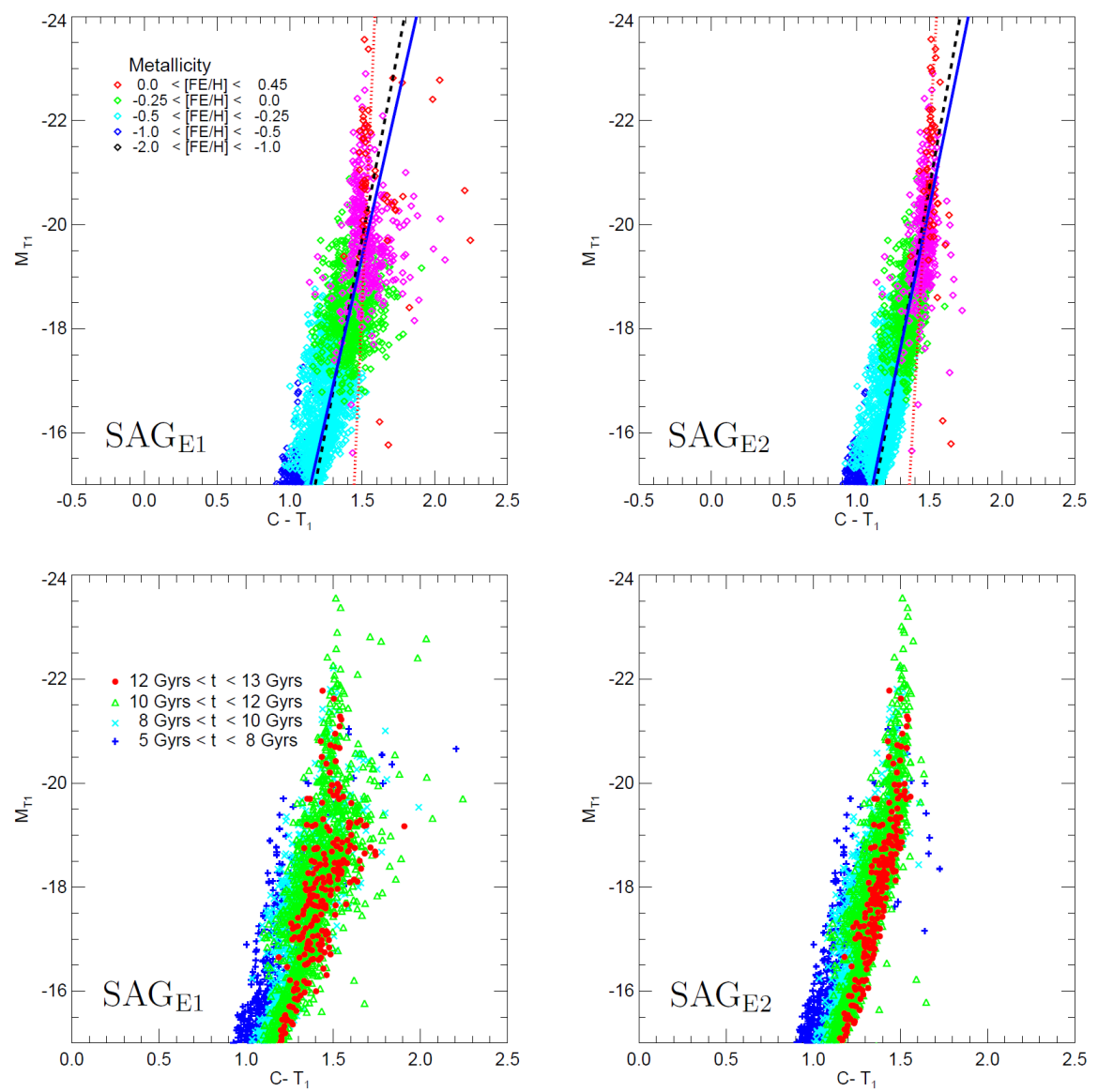

Figura 6.2: Comparación de las RCM del cúmulo de referencia, C153, en el sistema de Washington $\left(C-T_{1}\right)$ vs. $T_{1}$, obtenidas a partir de $\mathrm{SAG}_{\mathrm{E} 1}$, con el modelo de extinción por polvo original (paneles izquierdos). Las RCM resultantes de $\mathrm{SAG}_{\mathrm{E} 2}$, con la nueva formulación adoptada (paneles derechos). Los paneles superiores e inferiores muestran la misma comparación pero, los símbolos en colores siguen la distribución de metalicidad y de edad, respectivamente. 
quienes estudian el efecto del modelo de extinción sobre las distribuciones de galaxias seleccionadas por color, la función de luminosidad en la banda $B$ y las propiedades de clustering de las galaxias. Estos autores concluyen que un modelo con extinción reduce el número de galaxias azules. La función de correlación $\xi(r)$ presenta mayor amplitud y una pendiente más empinada. Hallan también, un mejor ajuste en el extremo brillante de la función de luminosidad en la banda $B$, obteniéndose en general, un mejor acuerdo con las observaciones que en el caso de un modelo sin extinción.

En la comparación de la RCM con y sin extinción, usamos como referencia la RCM obtenida por la versión del modelo $\mathrm{SAG}_{\mathrm{E} 1}$, que considera el efecto de extinción por polvo descripto en la capítulo 2.3.8 y que hemos utilizado a lo largo de este trabajo. Luego, en una segunda etapa, comparamos las RCM obtenidas según las distintas implementaciones del efecto de extinción, a las que llamamos " $\mathrm{RCM} \mathrm{E}_{\mathrm{E} 1}$ " y " $\mathrm{RCM}_{\mathrm{E} 2}$ " según hayan sido construidas a partir de las galaxias generadas por los modelos $\mathrm{SAG}_{\mathrm{E} 1}$ y $\mathrm{SAG}_{\mathrm{E} 2}$, respectivamente. A fin de identificar los efectos del polvo en los colores y magnitudes, la población de galaxias sinéticas en los distintos casos estudiados se genera a partir de SAG sin modificar los parámetros que caracterizan el modelo, presentados en el captítulo 2.

En lo que resta de este capítulo se muestra la influencia de los distintos aspectos que aquí analizamos (extinción por polvo, estrellas en la fase TP-AGB, formación estelar), sobre el cúmulo de referencia $\mathrm{C} 15_{3}$ (g51). Si bien en todos los cúmulos masivos se observan las mismas características, elegimos uno particular donde los cambios se visualizan claramente.

En la figura 6.1 se compara la $\mathrm{RCM}_{\mathrm{E} 1}$ del cúmulo $\mathrm{C} 15_{3}$ en el sistema de Washington (panel superior izquierdo) con la RCM resultante de excluir el efecto de extinción por polvo en SAG (panel inferior izquierdo). Como cabía esperar, los colores de las galaxias de la RCM sin corrección por polvo tienden a ser más azules y las magnitudes más negativas (lo que corresponde a galaxias más brillantes), ya que la luminosidad no está atenuada; por consiguiente, la RCM se desplaza hacia arriba y hacia la izquierda con respecto a $\mathrm{RCM}_{\mathrm{E} 1}$. La escala de magnitudes está extendida hasta el valor $\mathrm{M}_{\mathrm{T}_{1}}=-26$ para poder visualizar las galaxias más brillantes de la RCM cuando no se aplica el efecto de extinción por polvo. Con el fin de apreciar el comportamiento en el extremo brillante, se muestra la RCM sin extinción por polvo en una escala menor en los colores (panel inferior izquierdo). Es interesante notar que el quiebre en el extremo brillante está presente, aunque el mismo corresponde a otra magnitud de quiebre distinta de $\mathrm{M}_{\mathrm{T}_{1}}=-20$, dado el desplazamiento de la RCM hacia luminosidades mayores.

Las diferentes líneas, azul llena, roja punteada y negra de trazos que aparecen en la figura 6.1 corresponden a los ajustes por mínimos cuadrados realizados al extremo débil, brillante y a toda la RCM, respectivamente (ver capítulo 3). Estos ajustes son también trazados para las distintas RCM que se muestran en el resto de este capítulo. Como ya se mencionó oportunamente, en el sistema de Washington el color $\left(C-T_{1}\right)$ se caracteriza por una gran sensibilidad a la metalicidad de las galaxias (Forbes \& Forte, 2001). En las RCM mostradas en esta figura, las galaxias se representan mediante símbolos de colores codificados de acuerdo a la abundancia de hierro, $[\mathrm{Fe} / \mathrm{H}]$, de las mismas.

En la figura 6.2, se comparan las relaciones $\mathrm{RCM}_{\mathrm{E} 1}$ (panel superior izquierdo) y $\mathrm{RCM}_{\mathrm{E} 2}$ (panel superior derecho), ambas correspondientes al sistema de Washington. Como puede verse, el modelo $\mathrm{SAG}_{\mathrm{E} 2}$ reduce la dispersión de la relación color magnitud, especialmente en el extremo débil. Nuevamente, los símbolos de colores representan galaxias en distintos rangos de metalicidad. En los paneles inferiores de esta figura, se muestran las mismas RCM presentadas en los paneles superiores, pero los símbolos de distintos colores representan galaxias en distintos rangos de edad. Como se puede apreciar, al aplicar el modelo $\mathrm{SAG}_{\mathrm{E} 2}$ surge una estrecha correlación entre la edad y los colores de las galaxias, que resulta más definida que con el modelo $\mathrm{SAG}_{\mathrm{E} 1}$. En el extremo brillante de la $\mathrm{RCM}_{\mathrm{E} 2}$, encontramos que la mayoría de las galaxias comparte un mismo rango de edades $\left(1,0 \times 10^{10} \mathrm{yr}<t<1,2 \times 10^{10} \mathrm{yr}\right)$, de acuerdo con los resultados presentados en el capítulo 3. 
Cuadro 6.1: Pendientes de las rectas de ajuste al extremo débil de la RCM, al brillante y a toda la relación, calculadas mediante el método de mínimos cuadrados. Las RCM corresponden al cúmulo $\mathrm{C}_{1} 5_{3}$, obtenidas en diferentes bandas fotométricas a partir de los códigos $\mathrm{SAG}_{\mathrm{E} 1 \text { y }} \mathrm{SAG}_{\mathrm{E} 2}$ que consideran los modelos de extinción por polvo original y nuevo, respectivamente. En todos los casos, se calcula el ángulo de separación entre las rectas de ajuste al extremo débil y al brillante, $\Delta_{D-B}$

\begin{tabular}{l|cccc|cccc} 
& \multicolumn{4}{|c|}{ RCM $_{\mathrm{E} 1}$} & \multicolumn{4}{c}{$\mathrm{RCM}_{\mathrm{E} 2}$} \\
\hline & Ext. débil & Ext. brillante & Toda la RCM & $\Delta_{D-B}$ & Ext. débil & Ext. brillante & Toda la RCM & $\Delta_{D-B}$ \\
\hline$R$ vs. $(B-R)$ & -18.92 & -69.01 & -21.96 & $2^{\circ} 11^{\prime}$ & -19.96 & -64.35 & -22.21 & $1^{\circ} 58^{\prime}$ \\
$V$ vs. $(V-I)$ & -25.71 & -65.58 & -30.91 & $1^{\circ} 21^{\prime}$ & -28.15 & -109.79 & -32.13 & $1^{\circ} 30^{\prime}$ \\
$T_{1}$ vs. $\left(C-T_{1}\right)$ & -12.35 & -63.20 & -14.63 & $3^{\circ} 43^{\prime}$ & -13.67 & -48.12 & -15.51 & $2^{\circ} 59^{\prime}$ \\
$R$ vs. $(U-R)$ & -9.31 & -25.58 & -10.48 & $3^{\circ} 53^{\prime}$ & -9.56 & -24.86 & -10.43 & $3^{\circ} 40^{\prime}$
\end{tabular}


Debido a la sensibilidad de las diferentes bandas fotométricas a propiedades de las galaxias, tales como la edad y la metalicidad, visualizamos el efecto de los dos modelos de extinción implementados en SAG en todos los planos color magnitud estudiados en el capítulo 3. Las comparaciones correspondientes en los planos $R$ versus $(U-R), R$ versus $(B-R)$, y $V$ versus $(V-I)$ se muestran, respectivamente, en los paneles superiores, medios e inferiores de la figura 6.1.1. Cuantificamos las diferencias existentes en las RCM debidas a los distintos efectos de extinción, mediante la comparación de las pendientes de las rectas de ajustes por mínimos cuadrados al extremo débil, al brillante y a toda la relación. Calculamos el ángulo $\Delta_{D-B}$ entre las rectas de ajuste al extremo débil y brillante para cada banda fotométrica. Los resultados de los ajustes se vuelcan en la tabla 6.1. Observando los valores del ángulo $\Delta_{D-B}$ de la tabla 6.1 , notamos que la nueva implementación reduce el ángulo en todas las bandas, salvo en el plano $V$ vs. $V-I$, donde hay un aumento de 0,1 minutos de arco. La disminución más significativa ocurre en el plano $T_{1}$ vs. $\left(C-T_{1}\right)$ (ver figura 6.2 ). En todos los casos, el quiebre de las $\mathrm{RCM}_{\mathrm{E} 2}$ en el extremo brillante está presente, aunque es menos pronunciado que en las correspondientes $\mathrm{RCM}_{\mathrm{E} 1}$. Cabe destacar que encontramos el quiebre aún cuando no se aplica ningún modelo de extinción por polvo a las galaxias, tal como se ve en el panel inferior de la figura 6.1 .

\subsubsection{Efectos sobre la Relación Luminosidad Metalicidad}

Luego de analizar los efectos del nuevo modelo de extinción sobre los colores de las galaxias que forman la RCM, nos interesa estudiar las diferencias causadas por este modelo sobre la relación luminosidad metalicidad (RLM) de las mismas galaxias que forman la RCM. En la figura 6.1.1 ACA QUEDO MAL TAMBIEN, se muestra la comparación entre las RLM según resultan de la aplicación del modelo $\mathrm{SAG}_{\mathrm{E} 1}$ y $\mathrm{SAG}_{\mathrm{E} 2}$. Como puede verse, la RLM no resulta muy sensible al modelo de extinción utilizado, manteniendo en ambos casos las mismas caraterísticas generales, en buen acuerdo con las observaciones de Trager et al. (2000) y Mendel et al. (2009). La correlación entre las edades y la metalicidad de las galaxias es clara en el extremo más débil de la relación $\left(M_{T_{1}} \gtrsim-16\right)$, mientras que para galaxias pertenecientes al extremo brillante, es notoria la anticorrelación entre edad y metalicidad. Esta anticorrelación ya ha sido descripta en el capítulo 3; la misma está en concordancia con el análisis de resultados observacionales (Gallazzi et al., 2006).

En resumen, concluimos que la nueva implementación del modelo de extinción no afecta la presencia del quiebre en el extremo brillante de la RCM. De hecho, el quiebre está presente aun cuando no se aplica un modelo de extinción por polvo en SAG. Cuantificamos las diferencias entre las RCM resultantes de aplicar los modelos $\mathrm{SAG}_{\mathrm{E} 1}$ y $\mathrm{SAG}_{\mathrm{E} 2}$ en diferentes bandas fotométricas, mediante el cálculo de las pendientes de las rectas de ajuste por mínimos cuadrados a cada sección de la RCM, y mediante el ángulo de separación entre los ajustes al extremo brillante y débil. Encontramos que $\mathrm{RCM}_{\mathrm{E} 2}$ muestra una menor dispersión en los colores en todas las bandas, salvo en $V-I$, donde la dispersión es ligeramente mayor. Así mismo, la anticorrelación entre la edad y la metalicidad hallada también en la RLM de las galaxias pertenecientes al extremo brillante de la $\mathrm{RCM}_{\mathrm{E} 2}$ permite concluir, como hiciéramos en el capítulo 3, que el factor de mayor peso en la determinación de los colores de las galaxias que pueblan la RCM por encima del quiebre es la metalicidad de la mismas.

\subsection{Efectos de la fase TP-AGB en las galaxias}

Los modelos evolutivos de síntesis de poblaciones utilizados para reproducir la SED de las estrellas, cuentan con una serie de mejoras recientes en el tratamiento de fases evolutivas rápidas. Este es el caso de las estrellas pertenencientes a la Rama Asintótica 
de las Gigantes (AGB, según siglas en inglés, Asymptotic Giant Branch), en el diagrama HR (ver figura 6.5).

Luego de la quema central del hidrógeno en la secuencia principal y la posterior quema central del helio en la rama horizontal, las estrellas de masas menores a $8 M_{\odot}$ entran en la fase AGB. En esta etapa, las estrellas están caracterizadas por un núcleo inerte de carbono $(\mathrm{C})$ y oxígeno $(\mathrm{O})$, rodeado de dos capas concéntricas que queman helio $(\mathrm{He})$ e hidrógeno $(\mathrm{H})$. Las estrellas experimentan una serie de inestabilidades en la capa que quema He, conocidas como pulsos térmicos. En la fase de pulsos térmicos en las AGB, (TP-AGB, de su sigla en inglés, Thermally Pulsating), tiene lugar una serie de procesos de interés para el enriquecimiento químico de las galaxias.

Las estrellas en la fase AGB pierden una cantidad de masa mayor que en etapas anteriores de la evolución. La pérdida de masa gobierna la duración de la fase AGB, siendo las estrellas más luminosas aquéllas que pierden mayores fracciones de masa. La síntesis de elementos en las estrellas durante la fase AGB refleja la coexistencia de las capas que queman $\mathrm{H}$ y He y la interacción entre las mismas. Esta síntesis es dominada por un complejo ciclo de mezclas convectivas y cambios periódicos en las condiciones termodinámicas, inducidas por eventos de quema en flash (flash-burning) en la capa de He. Las interacción entre las dos capas que queman $\mathrm{H}$ y He llevan al enriquecimiento de la superficie estelar en nitrógeno $(\mathrm{N})$ y/o carbono. A su vez, esta misma interacción permite la generación de neutrones y la posterior producción de elementos más pesados que el hierro (Fe), mediante procesos de captura lenta de neutrones (slow processes, s-processes).

En las etapas finales de su evolución, la pérdida de masa puede desprender casi completamente el manto de la estrella; la presión de radiación sobre los granos de polvo de carbono en la superficie lleva a la generación de fuertes vientos estelares que devuelven parte de la masa estelar al medio interestelar. Esto culmina en la formación de una Nebulosa Planetaria, dejando como remanente el cadáver de una estrella enana blanca C-O. De este modo, las estrellas AGB producen diferentes elementos químicos, contribuyendo al enriquecimiento químico del ISM por acción de los vientos y las grandes pérdidas de masa, contaminando el ISM con elementos de producción primaria sintetizados a partir del $\mathrm{H}$ y He, como el ${ }^{12} \mathrm{C},{ }^{14} \mathrm{~N}$ y ${ }^{22} \mathrm{Ne}$. Elementos de producción secundaria, como el ${ }^{26} \mathrm{Al}$ (radiactivo) resultan de interés por su importancia en la astronomía de rayos $\gamma$ y para la interpretación de granos presolares (Forestini et al., 1991). Como resultado de la pérdida de masa, el espectro de las AGB en el infrarrojo está dominado por distintas clases de partículas de polvo (Blommaert et al., 2006).

Muchas son las razones por las cuales es importante considerar las estrellas AGB en los modelos de evolución de galaxias. Por un lado, las estrellas AGB de C son trazadoras de las distribuciones estelares del halo (Demens et al., 2004), indicando de esta manera el tamaño y la dinámica del sistema. Se identifican poblaciones estelares de edades intermedias a través de ellas, proporcionando así un mapa de la formación estelar reciente (0,5-1 Gyrs) en la región donde se encuentran. Ha sido demostrado que para poblaciones de edades en el rango 0,2-2 Gyrs, hasta el 40 por ciento de la luminosidad bolométrica es proporcionada por estrellas en la fase TP-AGB. Más del 80 por ciento de la luminosidad infrarroja más allá de 1 micrón, donde la SED alcanza su máximo, proviene de estas estrellas y domina el espectro en la banda $K$ (Grebel et al., 1999). El correcto modelado de los elementos químicos que forman parte del ISM es crucial en los modelos de formación de galaxias, debido a que las tazas de enfriamiento del gas dependen sensiblemente de la metalicidad (Sutherland \& Dopita, 1993). Por otro lado, la influencia de las pérdidas de energía se reflejan en las tasas de formación estelar. La energía liberada podría contribuir a regular la SFR aumentando la temperatura y desmantelando las nubes de gas frío de la galaxia, eventualmente reforzando la formación de vientos galácticos que transportan material enriquecido al medio intergaláctico (Hopkins et al., 2011).

Para nuestro estudio, la importancia de considerar las estrellas en la AGB reside en que poblaciones estelares en un pequeño rango de edades ( $\sim 1$ Gyr) forman una AGB 
Cuadro 6.2: Pendientes de las rectas de ajuste al extremo débil de la RCM, al brillante y a toda la relación, calculadas mediante el método de mínimos cuadrados.

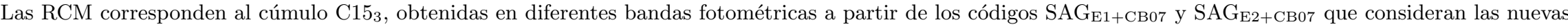
librerías de síntesis poblacional combinadas con los modelo de extinción por polvo original y nuevo, respectivamente. En todos los casos, se calcula el ángulo de separación entre las rectas de ajuste al extremo débil y al brillante, $\Delta_{D-B}$.

\begin{tabular}{|c|c|c|c|c|c|c|c|c|}
\hline & \multicolumn{4}{|c|}{$\mathrm{RCM}_{\mathrm{E} 1+\mathrm{BC} 07}$} & \multicolumn{4}{|c|}{$\mathrm{RCM}_{\mathrm{E} 2+\mathrm{BC} 07}$} \\
\hline & Ext. débil & Ext. brillante & Toda la RCM & $\Delta_{D-B}$ & Ext. débil & Ext. brillante & Toda la RCM & $\Delta_{D-B}$ \\
\hline$R$ vs. $(B-R)$ & -23.02 & -76.92 & -26.33 & $1^{\circ} 44^{\prime}$ & -24.55 & -65.86 & -26.72 & $1^{\circ} 27^{\prime}$ \\
\hline$V$ vs. $(V-I)$ & -31.16 & -88.58 & -38.51 & $1^{\circ} 11^{\prime}$ & -34.83 & -206.68 & -40.33 & $1^{\circ} 22^{\prime}$ \\
\hline$T_{1}$ vs. $\left(C-T_{1}\right)$ & -15.07 & -151.66 & -18.27 & $3^{\circ} 25^{\prime}$ & -16.90 & -79.34 & -19.49 & $2^{\circ} 39^{\prime}$ \\
\hline$R$ vs. $(U-R)$ & -10.09 & -25.23 & -11.23 & $3^{\circ} 23^{\prime}$ & -10.36 & -23.47 & -11.14 & $3^{\circ} 40^{\prime}$ \\
\hline
\end{tabular}


brillante y bien poblada. Por ello, esta fase constituye un excelente indicador de la edad, que puede ser usado para romper la degeneración edad/metalicidad que afecta el análisis de los espectros estelares.

\subsubsection{Nuevos modelos evolutivos de síntesis de poblaciones}

La estimación de la edad y la masa de las poblaciones estelares depende de manera crítica de los ingredientes usados en los modelos evolutivos de síntesis poblacional. Maraston et al. (2006) ha demostrado que el tratamiento de la fase TP-AGB es fuente de enormes dicrepancias a la hora de predecir la edad y la masa de poblaciones estelares a alto redshift $(1,4<z<2,7)$. El modelado de la fase AGB es difícil debido a las inestabilidades numéricas que surgen como consecuencia de los pulsos térmicos y los intensos vientos estelares.

Como hemos visto en el capítulo 2, las librerías sintéticas incorporadas originalmente en el modelo SAG pertenecen a Bruzual \& Charlot (2003). Alternativamente, el trabajo de Maraston (2005) (M05) constituye un ejemplo de la implementación de un modelo de síntesis de poblaciones con el tratamiento detallado de la etapa TP-AGB. Dicho modelo predice edades menores en las poblaciones estelares y una reducción de hasta el 60 por ciento en las masas de las mismas, con respecto al modelo de BC03. Las galaxias, pues, resultan más brillantes y menos enrojecidas en la banda del infrarojo cercano. Debido a que la etapa TP-AGB es dominante para los primeros Gyrs de las poblaciones estelares, su inclusión en el modelado provoca que las galaxias se vean más rojas a edades más tempranas que lo que usualmente se predice en modelos sin esta implementación (v.g. el modelo de BC03). Por ello, la conocida correlación "rojo-viejo", debe reinterpretarse a la luz de estos nuevos resultados.

El trabajo presentado por Charlot \& Bruzual (2007) es una actualización reciente del modelo BC03 con la incorporación de los tracks evolutivos provistos por Marigo \& Girardi (2007), que incluyen el tratamiento de la fase TP-AGB. En adelante nos referiremos a este modelo como CB07. En este nuevo modelo, las masas y edades de las poblaciones estelares resultan menores que en el modelo $\mathrm{BC} 03$, en concordancia con los resultados hallados por Maraston (2005).

Bruzual (2007) analiza extensivamente las diferencias entre su nuevo y viejo modelo, CB07 y BC03, respectivamente. Encuentra que los colores $B-V$ resultantes de los modelos BC03 y CB07 son idénticos para todas las edades de las poblaciones estelares modeladas. En $V-K$, a edades tempranas y tardías, los modelos coindicen, pero en edades intermedias los colores del modelo CB07 son considerablemente más rojos que los hallados mediante la aplicación de BC03. A edades tardías, estos modelos parecen ajustar bien las observaciones de ETGs cercanas, mientras el modelo M05 predice colores muy azules (ver figura 6.6).

Dado que los modelos evolutivos de síntesis de poblaciones que incluyen la fase TPAGB predicen edades, masas y contenidos de polvo menores en las galaxias, estamos interesados en estudiar el impacto de los mismos en la RCM resultante de acoplar las nuevas librerías espectrales de BC07 al modelo SAG.

\subsubsection{Fase TP-AGB en modelos semianalíticos}

Los trabajos de Tonini et al. $(2009,2010)$ constituyen una de las primeras aplicaciones de los modelos evolutivos de síntesis de poblaciones con la inclusión de la etapa TP-AGB en los códigos semianalíticos. En ambos trabajos, los autores utilizan el código GalICS (Hatton et al., 2005), que incluye el modelo de poblaciones estelares de M05. Para aislar el efecto de la TP-AGB en los colores, no se incluye en el modelo semianalítico la extinción por polvo (Tonini et al., 2009). En este trabajo, Tonini et al. muestran que a alto redhisft $(z=3)$ los colores $V-K$ están desplazados hacia el rojo en valores de hasta 2 magnitudes, 
comparativamente con los colores obtenidos por su mismo código sin la implementación de poblaciones estelares con TP-AGB. Este desplazamiento en los colores disminuye a media que el redshift aumenta, pero lo hace de manera no lineal. El cociente entre la masa y la luminosidad en la banda $K$ se desplaza hacia luminosidades mayores de hasta una magnitud, especialmente en las galaxias masivas. Si se deseara reproducir esta emisión en la banda $K$ sin incluir la fase TP-AGB, serían necesarias masas estelares mayores y poblaciones más envejecidas, produciendo, por ende, una SFR más elevada a alto $z$. Uno de los resultados más interesantes de estos trabajos es la predicción de que a alto redshift las galaxias con formación estelar activa tienen colores $V-K$ y luminosidades en $I R$ similares a las poblaciones locales que evolucionaron pasivamente.

Henriques et al. (2011) usan distintos modelos semianalíticos equipados con modelos de poblaciones estelares con y sin la fase TP-AGB, para explorar la discrepancia hallada con las observaciones de la función de luminosidad en el IR cercano de galaxias a alto redshift. Al parecer, dicha discrepancia con las observaciones desaparece al utilizar modelos que incluyen la implementación de M05. A su vez, Fontanot \& Monaco (2010) hallan que los colores observados de los objetos extremadamente rojos (EROs, por su sigla en inglés, Extremely Red Objects) se ajustan mejor a las predicciones de los modelos teóricos que implementan la etapa TP-AGB en la síntesis de poblaciones.

\subsubsection{Efectos de la fase TP-AGB en la RCM}

Para aislar la influencia de las nuevas librerías sintéticas de BC07 en los colores, edades y metalicidades de las galaxias que pueblan la RCM, utilizamos como referencia a la RCM obtenida de la aplicación del modelo SAG original. Esto quiere decir que en cada realización del modelo mantenemos la formulación de extinción por polvo descripta en la sección 2.3.8, y las librerías sintéticas de BC03. Etiquetamos a esta relación como

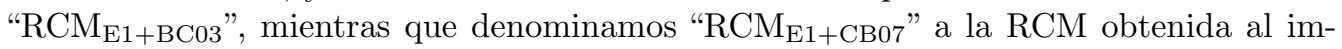
plementar las nuevas librerías de BC07 en el SAG, manteniendo el modelo de extinción original. Luego, acoplando al modelo SAG las dos nuevas implementaciones (modelo de extinción descripto en el capítulo 6.1 y nuevas librerías de BC07), resulta una RCM que se distingue con el rótulo " $\mathrm{RCM}_{\mathrm{E} 2+\mathrm{CB} 07}$ ". La comparación de estas tres relaciones en distintas bandas fotométricas $\left(C, T_{1} ; U, R ; V, I ; B, R\right)$ se presenta en las figuras 6.7 y 6.8 , donde los símbolos están codificados de acuerdo a la metalicidad de las galaxias. La figura 6.9 muestra el mismo conjunto de RCM en el sistema de Washington, identificando las galaxias por su edad. Cuantificamos las diferencias halladas entre las distintas relaciones obtenidas mediante el cálculo del ángulo $\Delta_{D-B}$ que forman las rectas de ajuste por mínimos cuadrados al extremo brillante y al débil de las RCM, para cada banda fotométrica. Los resultados de este cálculo se muestran en la tabla 6.2.

Del análisis de los valores de la tabla 6.2 y de las figuras 6.7 y 6.8 , resulta claro que la $\mathrm{RCM}_{\mathrm{E} 1+\mathrm{CB} 07}$ mantiene las características generales encontradas en el modelo original $\mathrm{RCM}_{\mathrm{E} 1+\mathrm{BC} 03}$, presentando el quiebre en el extremo brillante de la relación hacia colores más azules. Los valores de las pendientes correspondientes al cálculo de las rectas de ajuste de esta relación original se encuentran en la primera columna de la tabla 6.1. Comparando esta columna con la primera columna de la tabla 6.2 , podemos ver las diferencias en las pendientes de las rectas de ajuste resultantes de aplicar dos versiones del modelo $\mathrm{SAG}\left(\mathrm{SAG}_{\mathrm{E} 1+\mathrm{BC} 03}\right.$ y $\left.\mathrm{SAG}_{\mathrm{E} 1+\mathrm{CB} 07}\right)$, que sólo difieren en las librerías espectrales utilizadas. Notamos que el efecto de las nuevas librerías de síntesis poblacional, es el ángulo de diferencia entre las pendientes de ajuste del extremo débil y brillante. Este comportamiento es común en todas las bandas fotométricas, siendo más significativa en la banda $(B, R)$, como puede verse en la reducción del correspondiente ángulo $\Delta_{D-B}$. El mismo efecto se aprecia al comparar la segunda columna de la tabla 6.1 con la segunda columna de la tabla 6.2, donde se ponen en evidencia las diferencias debidas a las nuevas librerías espectrales, ahora con la implementación de la nueva extinción por polvo descripta en la sección 6.1. En esta comparación, se encuentra que el ángulo de separación 
$\Delta_{D-B}$ disminuye en todas las bandas, menos en la banda $(U, R)$, donde el ángulo $\Delta_{D-B}$ no cambia. En general, las variaciones de ángulo son tan pequeñas que las diferencias de pendiente no son estadísticamente significativas. Finalmente, al comparar la primera columna de la tabla 6.1 con la segunda columna de la tabla 6.2, vemos las diferencias reflejadas en la RCM que surgen de la aplicación del modelo semianalítico original, y de aquél que incluye las dos nuevas implementaciones vistas en este capítulo. El efecto sobre la RCM es una reducción en la diferencia entre los extremos de la RCM en las bandas $(B, R)$ y $(U, R)$. La misma se refleja en una reducción promedio del ángulo $\Delta_{D-B}$ de 0,40 segundos de arco. En la banda $(V, I)$ el ángulo no muestra ningún cambio, pero sí existe una reducción significativa en la banda $\left(C, T_{1}\right)$ de $1^{\circ} 40^{\prime}$.

\subsection{Nueva formulación para la formación estelar}

En el código semianalítico SAG, la formación estelar en los discos galácticos se produce a partir del gas frío disponible en ellos, ya sea en forma de brotes estelares durante procesos de fusiones o inestabilidades de disco, o en el modo pasivo. La versión de SAG con la que se obtuvieron los resultados presentados en los capítulos anteriores simula el proceso de formación estelar pasiva mediante la formulación presentada en el capítulo 2.3 (ecuaciones (2.3) y (2.4)). A este modelo se lo distingue con las siglas "SF${ }_{1}$ ". Teniendo en cuenta que éste considera además el modelo de extinción por polvo " $\mathrm{E}_{1}$ " y las librerías de síntesis poblacional de $\mathrm{BC} 03$, nos referimos a esta versión de $\mathrm{SAG}$ como $\mathrm{SAG}_{\mathrm{E} 1+\mathrm{BC} 03+\mathrm{SF} 1}$. Basándose en observaciones de Kennicutt et al. (1989), Croton et al. (2006) proponen un modelo alternativo de SF que establece una masa límite de gas frío debajo de la cual no tiene lugar la formación de estrellas. Esta masa crítica viene dada por la siguiente expresión

$$
m_{\text {crit }}=3,8 \times 10^{9}\left(\frac{V_{\text {vir }}}{200 \mathrm{kms}^{-1}}\right)\left(\frac{r_{\mathrm{disc}}}{10 \mathrm{kpc}}\right) M_{\odot}
$$

siendo $r_{\text {disc }}$ el radio de escala del disco de la galaxia y $V_{\text {vir }}$ la velocidad virial del halo de materia oscura en el que reside la misma. Cuando la masa de gas frío en la galaxia es mayor que el valor crítico $m_{\text {crit }}$, la tasa de formación estelar es

$$
\dot{m}_{*}=\alpha_{\mathrm{SF}}\left(m_{\text {cold }}-m_{\text {crit }}\right) / t_{\text {dyn,disc }} .
$$

El valor de la eficiencia $\alpha_{\mathrm{SF}}$ es un parámetro libre del modelo que se ajusta para que una fracción del 5 al 15 por ciento del gas se convierta en estrellas durante el tiempo dinámico, $\mathrm{t}_{\mathrm{dyn} \text {,disc }}=\mathrm{r}_{\text {disc }} / \mathrm{V}_{\text {vir }}$. Este nuevo modelado de la $\mathrm{SF}$ es identificado con con el rótulo $\mathrm{SF}_{2}$, para distinguirlo del $\mathrm{SF}_{1}$ original. $\mathrm{Al}$ mantener las implementaciones de extinción por polvo y librerías sintéticas originales, y agregar la nueva formulación de $\mathrm{SF}$, obtenemos la versión $\mathrm{SAG}_{\mathrm{E} 1+\mathrm{BC} 03+\mathrm{SF} 2}$. Cabe destacar que al aplicar esta versión del código no se altera el valor del parámetro de eficiencia de formación estelar que permite lograr una buena calibración de la versión original de SAG. La ventaja de esta nueva implementación es que el gas frío de las galaxias con masas menores que $10^{10} M_{\odot}$ no resulta tan altamente contaminado, permitiendo obtener una relación masa metalicidad en muy buen acuerdo con tendencia marcada por las observaciones, que no era logrado con la formulación original de SF (ver figura 6.10). Este hecho es debido a la supresión de la formación estelar en galaxias con poco contenido de gas frío. 


\subsubsection{Influencia de la nueva formulación de SF en la RCM}

Aplicando la misma metodología de trabajo utilizada en las secciones anteriores, se analizan las RCM resultantes de combinar la nueva implementación considerada para simular el proceso de formación estelar, con los dos conjuntos de librerías de síntesis poblacional presentadas en este trabajo, es decir, las RCM que se obtienen de los modelos semianalíticos $\mathrm{SAG}_{\mathrm{E} 1+\mathrm{BC} 03+\mathrm{SF} 2}$ y $\mathrm{SAG}_{\mathrm{E} 1+\mathrm{CB} 07+\mathrm{SF} 2}$. Calculamos las pendientes de las rectas de ajustes a las dos secciones de la RCM, y a la relación completa, y el ángulo $\Delta_{D-B}$ de separación entre las rectas de ajuste a los extremos débil y brillante. Los resultados obtenidos para cada banda fotométrica se presentan en la tabla 6.3. Al comparar los valores presentados en las dos columnas de esta tabla, se aprecia que, al combinar la nueva formulación de formación estelar con las nuevas librerías de síntesis poblacional, se reduce el ángulo $\Delta_{D-B}$ en todas las bandas en un valor promedio de $0^{\circ} 35$.

Las diferencias entre las RCM obtenidas con el modelo semianalítico original y las resultantes de implementar en SAG la nueva formulación de SF, manteniendo el resto de las implementaciones originales, pueden visualizarse comparando la primera columna de la tabla 6.1 con la primera columna de la tabla 6.3, que muestran los resultados de los

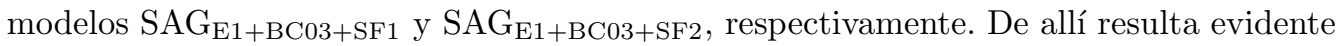
que el ángulo $\Delta_{D-B}$ aumenta en todas las bandas, siendo más significativo el aumento en la banda $(U, R)$. Este aumento entre las rectas de ajuste a los extremos débil y brillante radica en que el extremo brillante presenta un quiebre más pronunciado al aplicar la nueva formulación de SF.

Analizamos ahora la RCM que resulta de la incorporación de la nueva fórmula de SF al código SAG que a su vez considera las nuevas librerías de síntesis poblacional, esto es, $\mathrm{RCM}_{\mathrm{E} 1+\mathrm{CB} 07+\mathrm{SF} 2}$. La comparación de esta relación con la obtenida por el modelo original se presenta en la figura 6.11 para las bandas fotométricas $\left(C, T_{1}\right),(U, R)$ y $(V, I)$. De la inspección de los valores de la primera columna de la tabla 6.1 y la segunda columna de la tabla 6.3, podemos ver las diferencias entre el modelo original SAG y el modelo equipado con la nueva implentación de SF y las librerías de BC07. Las RCM obtenidas presentan una reducción del ángulo $\Delta_{D-B}$ en la banda $\left(C, T_{1}\right)$ y un aumento de la misma en el resto de las bandas analizadas. En la banda $(U, R)$, el aumento del ángulo es marcado $\left(\Delta_{F-B}=1^{\circ} 40^{\prime}\right)$.

Las propiedades de las galaxias tales como masas, metalicidades, edades y radios de escala de los discos que porporciona el código SAG constituyen los datos requeridos para la aplicación subsecuente del modelo de extinción por polvo y de las librerías espectrales que incluyen con detalle la emisión de estrellas en la fase TP-AGB. En tal sentido, estas implementaciones se aplican de manera "externa" a los cálculos inherentes a la evolución de las galaxias realizados por el código SAG, si bien están incluídas en el mismo. Dicho de otro modo, la aplicación de la extinción por polvo y el uso de librerías espectrales sólo modifican las magnitudes de las galaxias que ya son un resultado del código. A diferencia de este tipo de implementaciones "externas", las modificaciones en el proceso de formación estelar afectan cantidades básicas de las galaxias del modelo, tales como masas y metalicidades, las cuales son cantidades fundamentales que intervienen como condiciones iniciales en las ecuaciones diferenciales que son resueltas "dentro" del código. En particular, al considerar la formulación de formación estelar propuesta por Croton et al. (2006), encontramos que las galaxias en los intervalos de magnitud 1 y 2 de la RCM comparten la misma metalicidad a $z=0$. Este valor de metalicidad resulta $\sim 1$ dex más bajo que el obtenido del modelo original, como puede verse al comparar la figura $4.7 \mathrm{con}$ la figura 6.12 .

De lo anteriormente expuesto resulta claro que al cambiar la historia de formación estelar también se ven afectados la masa de gas y los radios de escala de los discos galácticos, que son cantidades de las cuales depende el nuevo modelo de extinción. Por lo tanto, al combinar este modelo con la formulación alternativa de formación estelar se producen cambios apreciables en los colores de las galaxias, lo cual conduce a una RCM que se aleja de la tendencia observada. En consecuencia, esta relación y otras propiedades 
Cuadro 6.3: Pendientes de las rectas de ajuste al extremo débil de la RCM, al brillante y a toda la relación, calculadas mediante el método de mínimos cuadrados.

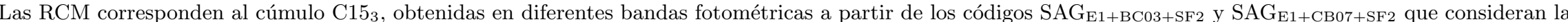
formulación alternativa de SF, combinada con el modelo de extinción por polvo original, y las librerías de síntesis poblacional original y nuevas, respectivamente, En todos los casos, se calcula el ángulo de separación entre las rectas de ajuste al extremo débil y al brillante, $\Delta_{D-B}$.

\begin{tabular}{|c|c|c|c|c|c|c|c|c|}
\hline & \multicolumn{4}{|c|}{$\mathrm{RCM}_{\mathrm{E} 1+\mathrm{BC} 03+\mathrm{SF} 2}$} & \multicolumn{4}{|c|}{$\mathrm{RCM}_{\mathrm{E} 1+\mathrm{BC} 07+\mathrm{SF} 2}$} \\
\hline & Ext. débil & Ext. brillante & Toda la RCM & $\Delta_{D-B}$ & Ext. débil & Ext. brillante & Toda la RCM & $\Delta_{D-B}$ \\
\hline$R$ vs. $(B-R)$ & -18.00 & 199.34 & -22.55 & $2^{\circ} 53^{\prime}$ & -21.34 & 191.13 & -26.74 & $2^{\circ} 22^{\prime}$ \\
\hline$V$ vs. $(V-I)$ & -25.85 & -889.84 & -33.74 & $2^{\circ} 09^{\prime}$ & -30.68 & 572.14 & -41.56 & $1^{\circ} 46^{\prime}$ \\
\hline$T_{1}$ vs. $\left(C-T_{1}\right)$ & -11.64 & 51.41 & -15.06 & $3^{\circ} 47^{\prime}$ & -13.64 & 38.90 & -18.35 & $2^{\circ} 72^{\prime}$ \\
\hline$R$ vs. $(U-R)$ & -8.89 & -147.36 & -10.70 & $6^{\circ} 01^{\prime}$ & -9.47 & -99.07 & -11.34 & $5^{\circ} 26^{\prime}$ \\
\hline
\end{tabular}


fundamentales de las galaxias (observables utilizados usualmente para calibrar el modelo; ver capítulo 2) no pueden ser reproducidos adecuadamente por la versión de SAG que accione simultáneamente todas las modificaciones presentadas en este capítulo (modelo de extinción, librerías de síntesis poblacional y modelo de formación estelar). Para obtener un buen acuerdo con propiedades y relaciones observadas, es necesario recalibrar el modelo, modificando el valor de los parámetros libres involucrados en los distintos procesos considerados.

Comparar modelos con diferentes parámetros no resulta de mucha utilidad a la hora de aislar el impacto en la RCM de diversos procesos físicos que afectan los colores, edades y metalicidades de las galaxias, como son la interacción de la SED de las galaxias con el polvo del ISM, la evolución estelar y la formación estelar. Por ello, creemos prudente concluir aquí este análisis y proyectar el trabajo futuro con una nueva versión del modelo SAG. 

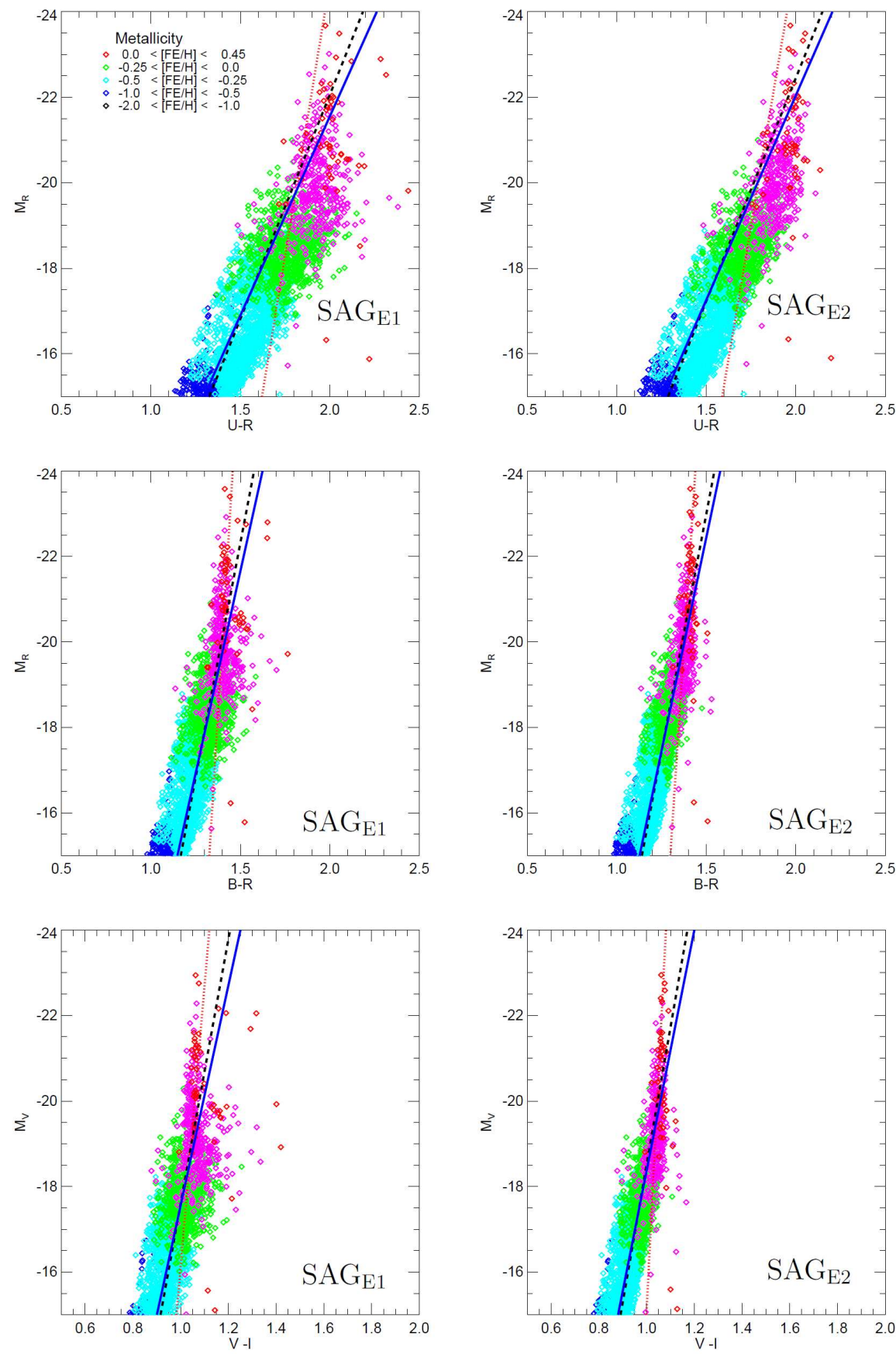

Figura 6.3: RCM del cúmulo $\mathrm{C} 15_{3}$ en los planos $R$ vs. $(U-R)$ (paneles superiores), $R$ vs. $(B-R)$ (paneles medios), $V$ vs. $(V-I)$ (paneles inferiores), obtenidas de aplicar el modelo SAG $\mathrm{E}_{\mathrm{E}}$ $\left(\mathrm{RCM}_{\mathrm{E} 1}\right.$, paneles izquierdos), y $\mathrm{SAG}_{\mathrm{E} 2}\left(\mathrm{RCM}_{\mathrm{E} 2}\right.$, paneles derechos), que consideran el modelo de extinción por polvo original y nuevo, respectivamente. En todas las figuras se grafican las rectas de ajustes por mínimos cuadrados realizados al extremo débil de la RCM (línea azul llena), brillante (línea roja punteada) y a toda la relación (línea negra de trazos). Los colores representan la distribución de metalicidad de las galaxias, como se indica en la figura. 

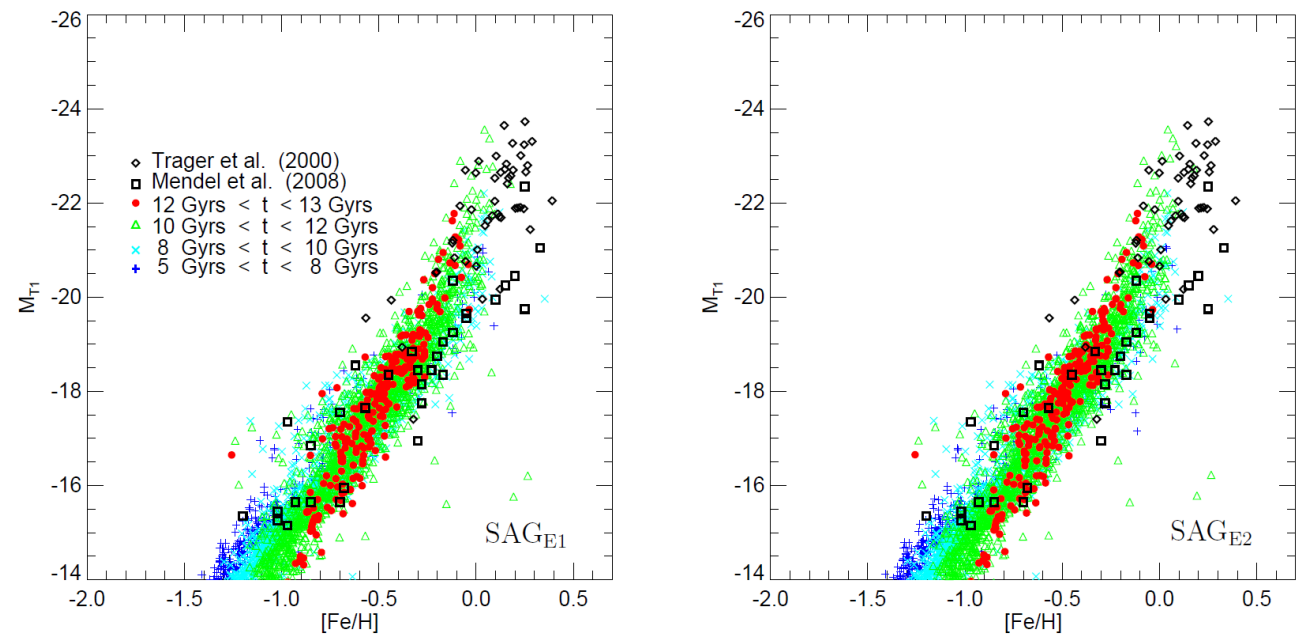

Figura 6.4: Comparación de las relaciones Luminosidad Metalicidad del cúmulo de refencia $\mathrm{C} 15_{3}$ resultantes de aplicar los modelos $\mathrm{SAG}_{\mathrm{E} 1}$ (panel izquierdo) y $\mathrm{SAG}_{\mathrm{E} 2}$ (panel derecho). Estas relaciones son construidas a partir de las mismas galaxias simuladas pertenecientes a la $\mathrm{RCM}_{\mathrm{E} 1}$ y $\mathrm{RCM}_{\mathrm{E} 2}$, respectivamente. Los símbolos de colores identifican diferentes rangos de edades de las galaxias; los diamantes negros representan las abundancias $[\mathrm{Fe} / \mathrm{H}]$ de la muestra de ETGs presentada por Trager et al. (2000), mientras que los cuadrados negros corresponden a ETGs del grupo NGC 5044 (Mendel et al., 2009).

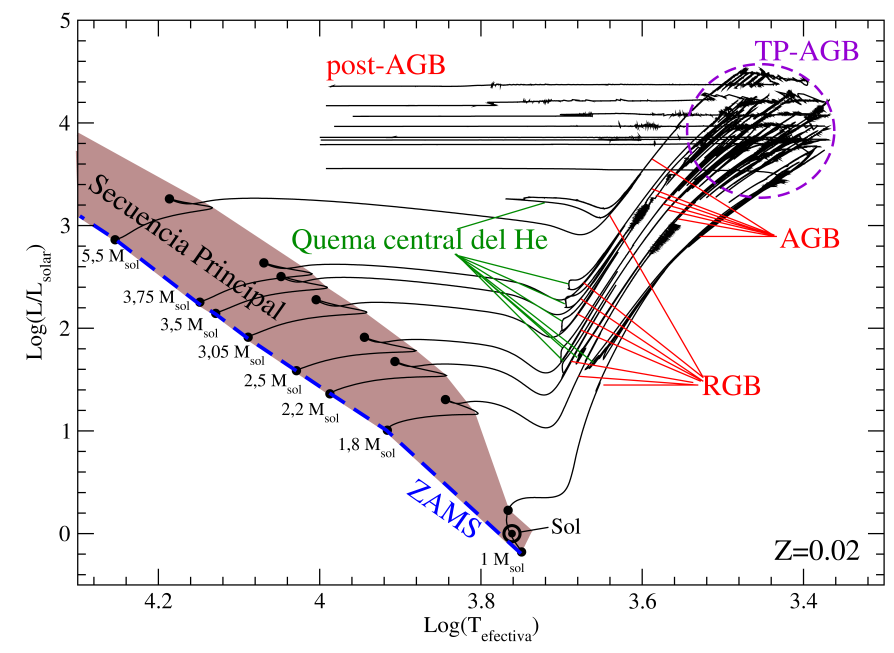

Figura 6.5: Diagrama Hertzsprung-Russell, donde se muestran diferentes etapas de la evolución de las estrellas que típicamente atraviesan la Rama Asintótica de las Gigantes; estas estrellas poseen masas bajas e intermedias $1 M_{\odot} \leq M \leq 9 M_{\odot}$, (Miller Bertolami, 2009). 


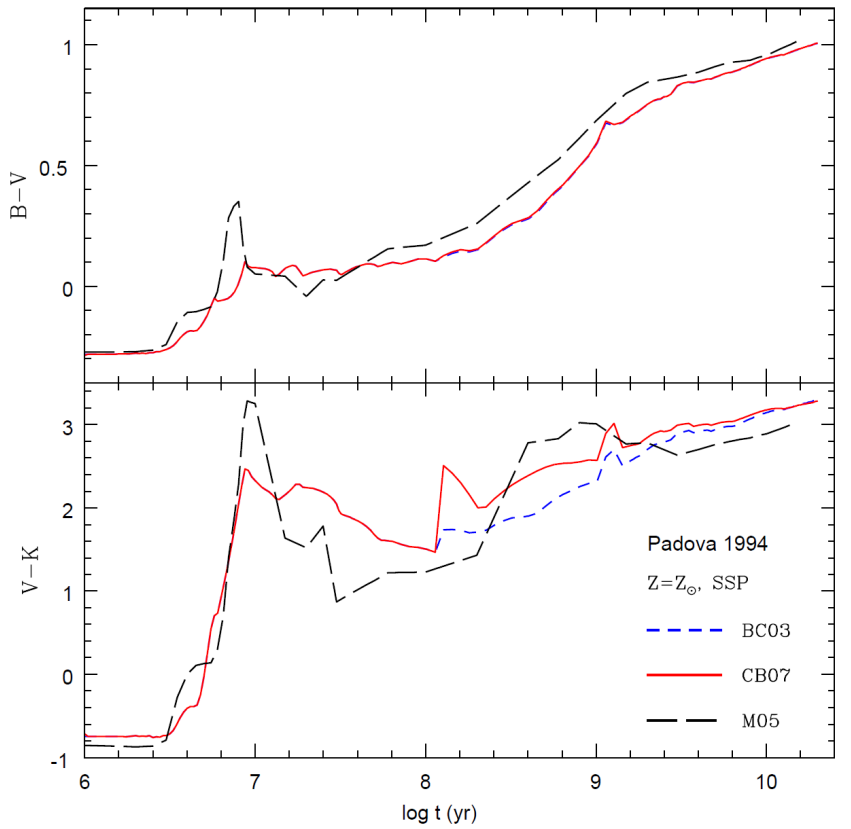

Figura 6.6: Comparación entre la evolución de los colores en las bandas $B-K$ y $V-K$ para los modelos de Charlot \& Bruzual (2007) (en línea roja) usando los tracks deMarigo \& Girardi (2007) que incluyen la implementación de la fase TP-AGB, con los modelos de Bruzual \& Charlot (2003) (en línea de trazos, azul), y el modelo de M05 (línea negra de trazos). 

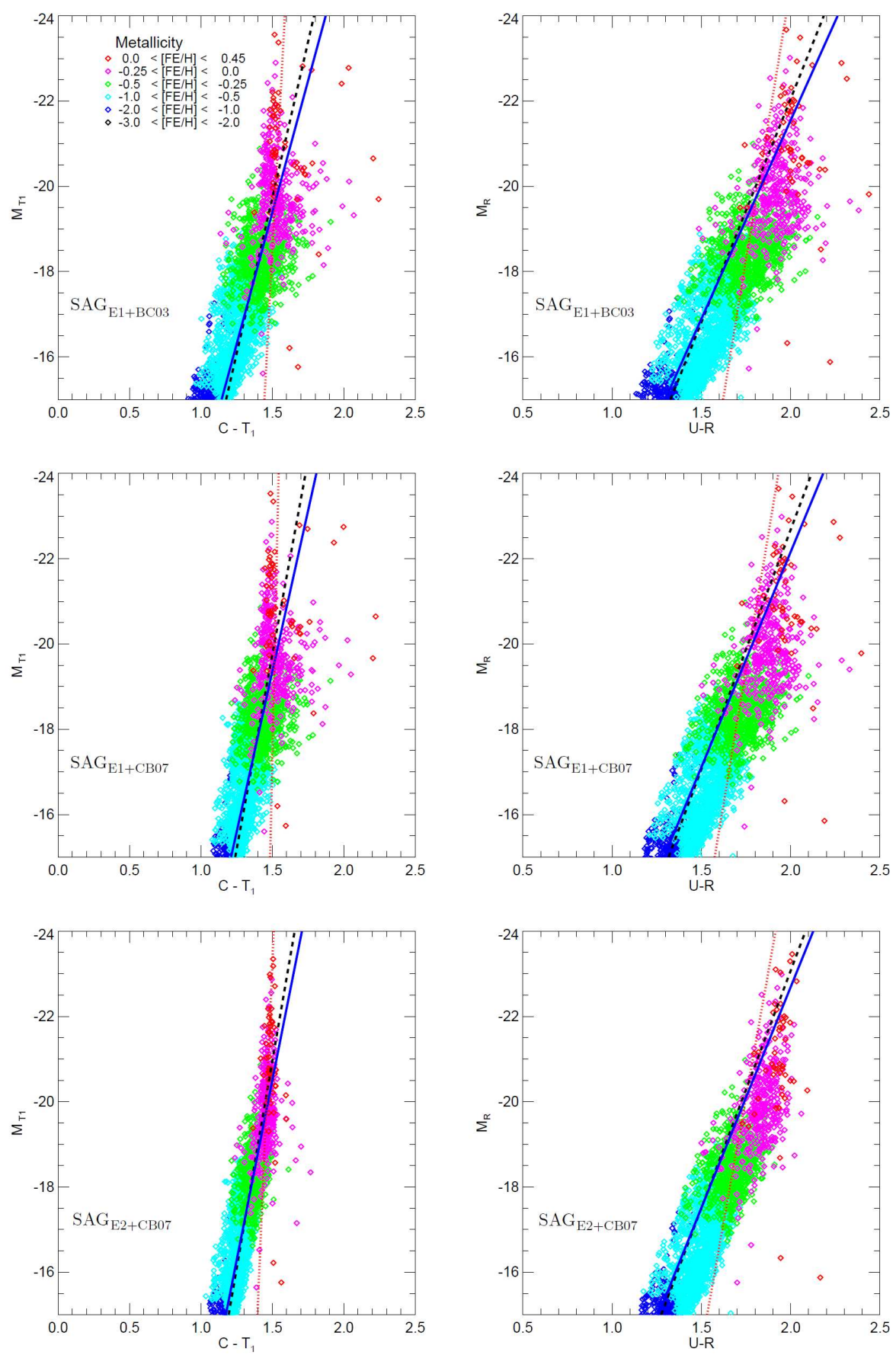

Figura 6.7: Se comparan las RCM del cúmulo de referencia, $\mathrm{C} 15_{3}$, en las bandas fotométricas $\left(C, T_{1}\right)$ (columna izquierda) y $(U, R)$ (columna derecha) obtenidas a partir de la aplicación de tres versiones de SAG: la original, sin ninguna de las nuevas implementaciones descriptas en este capítulo ( $\mathrm{RCM}_{\mathrm{E} 1+\mathrm{BC} 03}$, paneles superiores), la que considera las nuevas librerías de síntesis poblacional de $\mathrm{CB} 07$ ( $\mathrm{RCM}_{\mathrm{E} 1+\mathrm{CB} 07}$, paneles medios), y la que combina estas nuevas librerías con el nuevo modelo de extinción por polvo detallado en la seccion $6.1, \mathrm{RCM}_{\mathrm{E} 2+\mathrm{CB} 07}$ (panel inferior). 

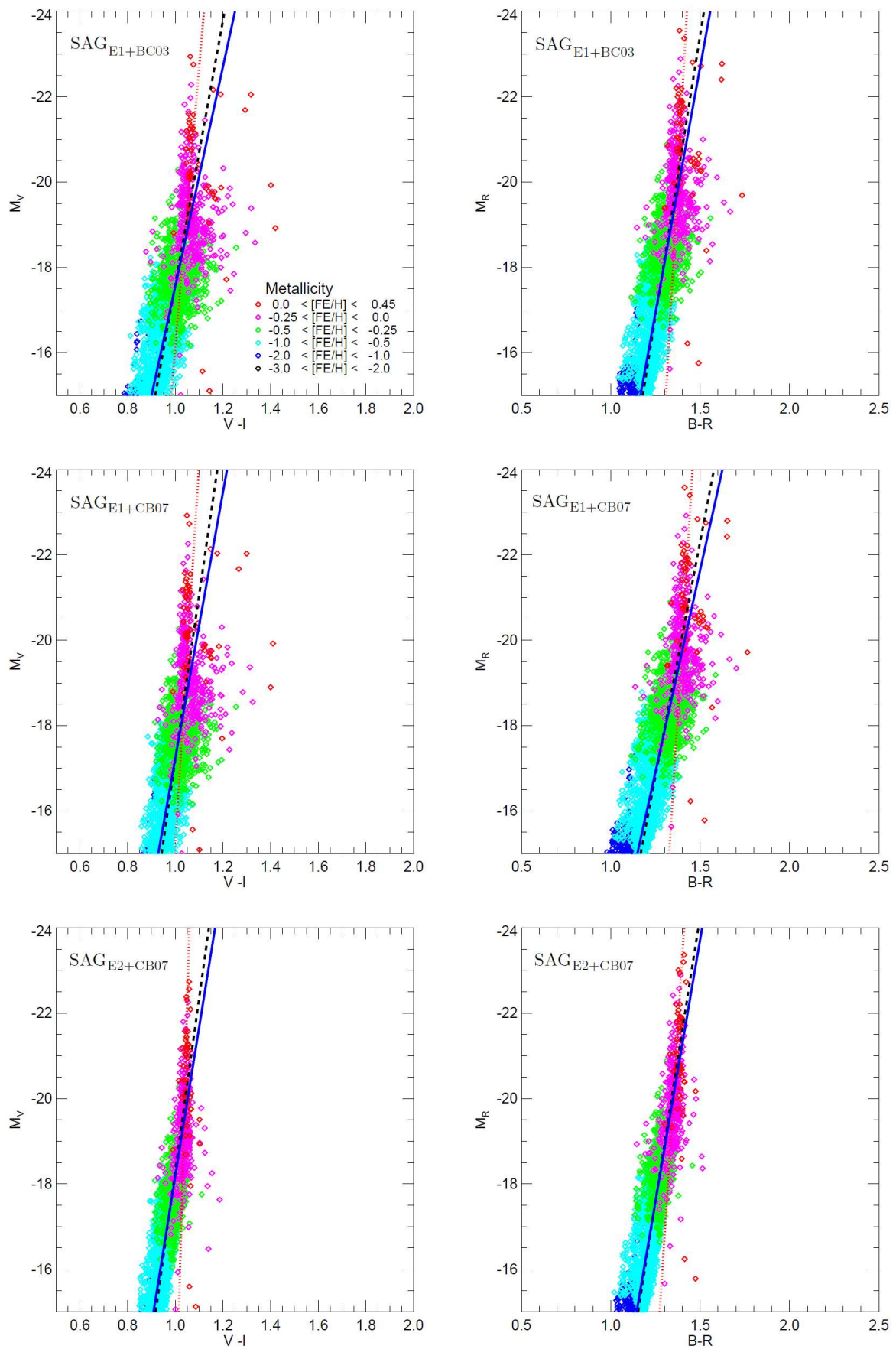

Figura 6.8: Idem a la figura 6.7 en las bandas fotométricas $(V, I)$ (columna izquierda) y $(B, R)$ (columna derecha). 

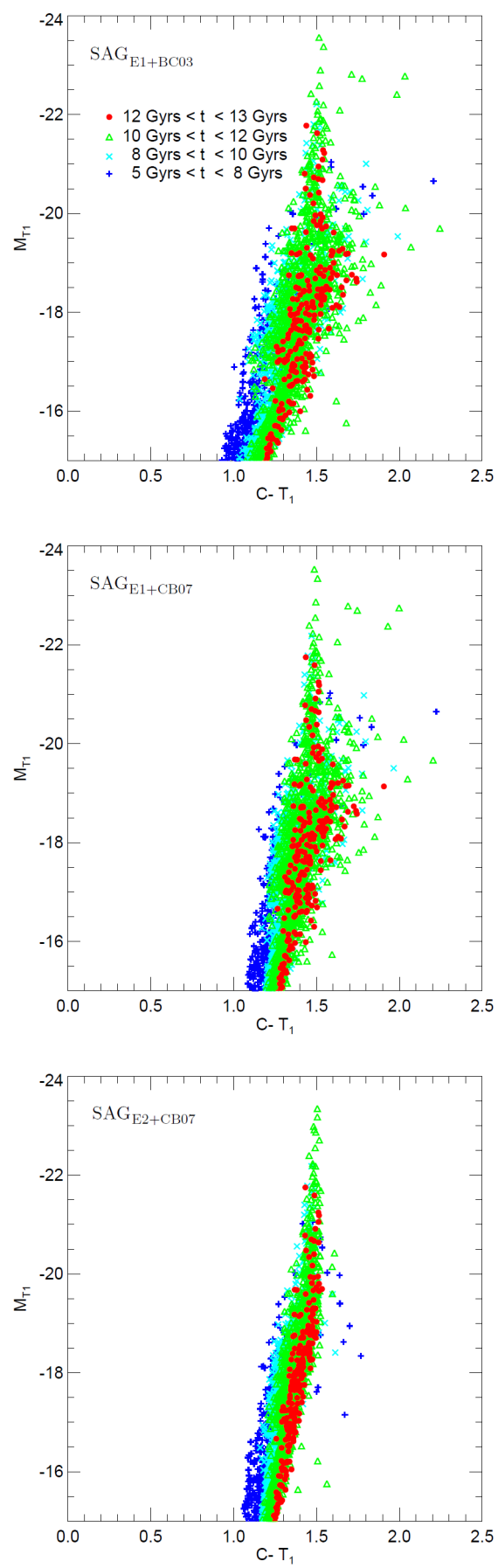

Figura 6.9: Se muestra la columna izquierda de la figura 6.7 (RCM en el sistema de Washington) con los símbolos que representan las galaxias coloreados de acuerdo a la edad de las mismas. 

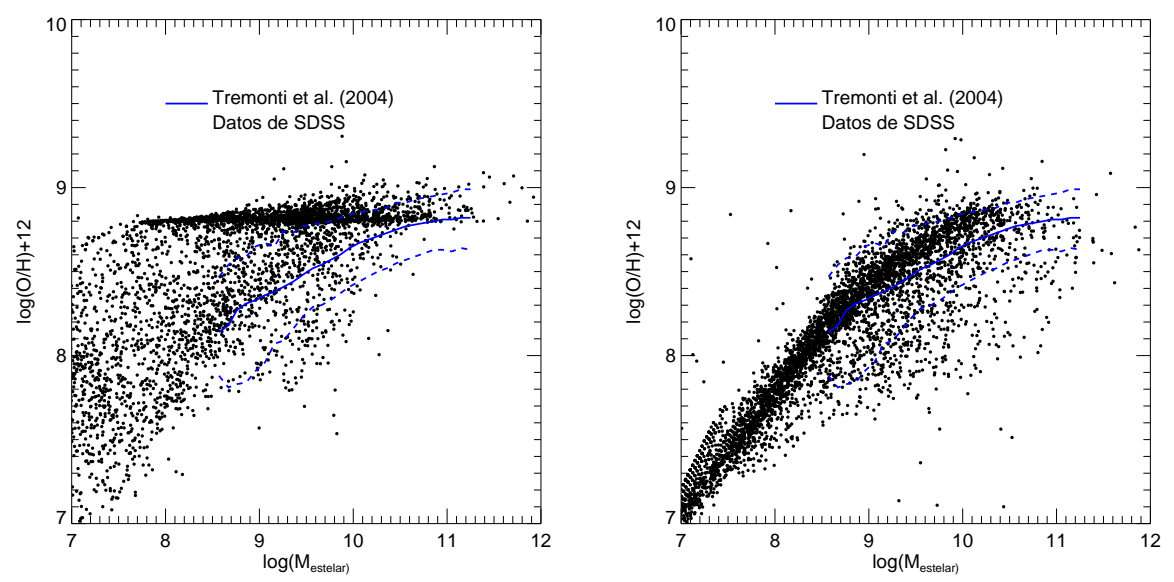

Figura 6.10: Relación masa metalicidad del cúmulo de referencia C15_3 obtenida a partir de la versión original de SAG: $\mathrm{SAG}_{\mathrm{E} 1+\mathrm{BC} 03+\mathrm{SF} 1}$ (panel izquierdo), y la que considera el modelo alternativo de SF dado por Croton et al. (2006): $\mathrm{SAG}_{\mathrm{E} 1+\mathrm{BC} 03+\mathrm{SF} 2}$ (panel derecho). 

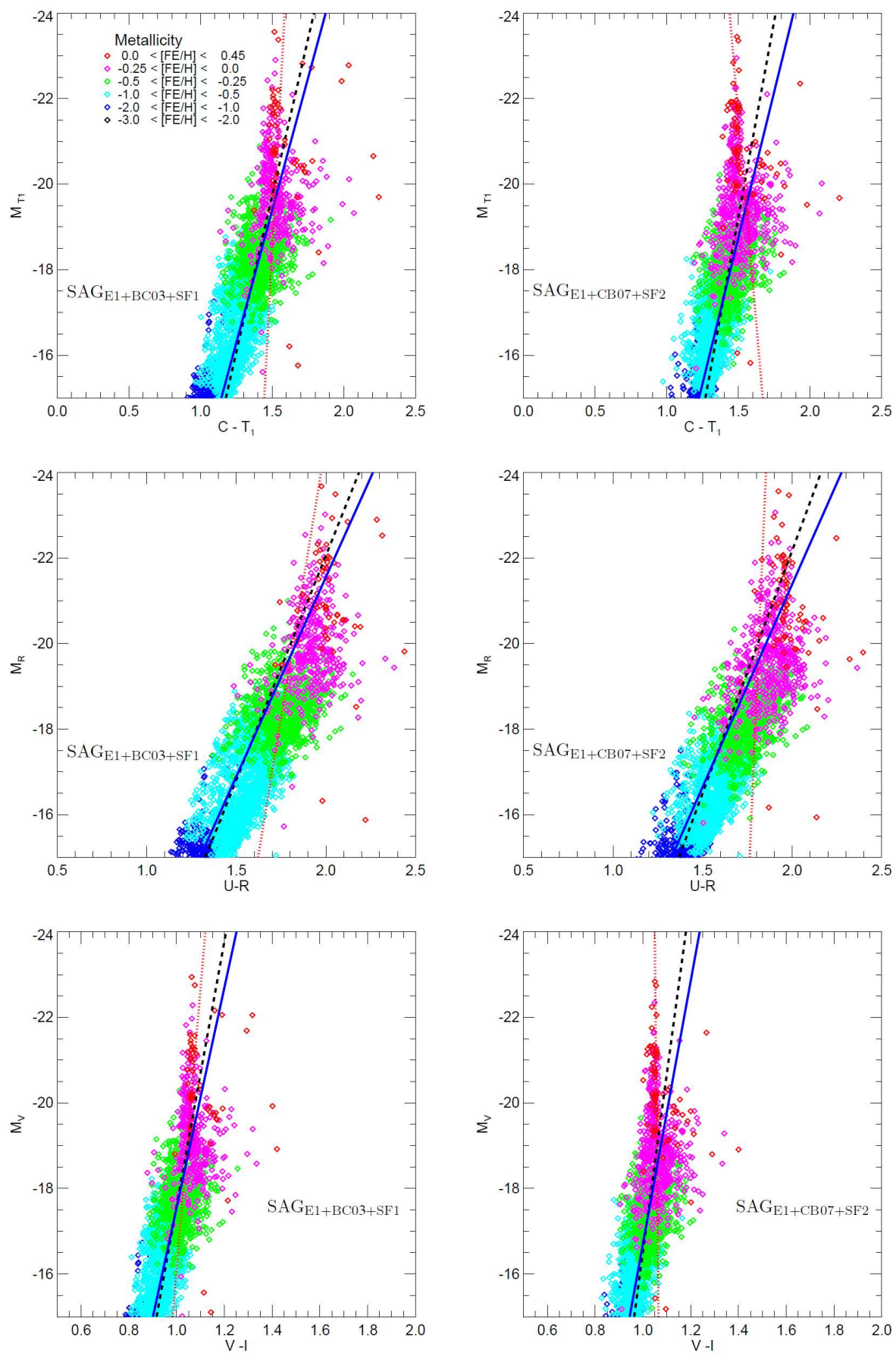

Figura 6.11: Comparación de las RCM del cúmulo $\mathrm{C} 15_{3}$ obtenidas a partir de la código semi-

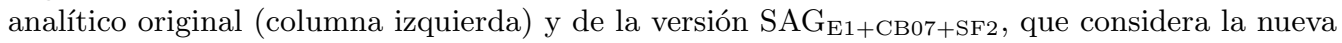
formulación de SF, combinada con las librerías de CB07 (columna derecha). Las RCM se muestran en las bandas fotométricas $(C, T 1)$ (paneles superiores), $(U, R)$ (paneles medios), y $(V, I)$ (paneles inferiores). 

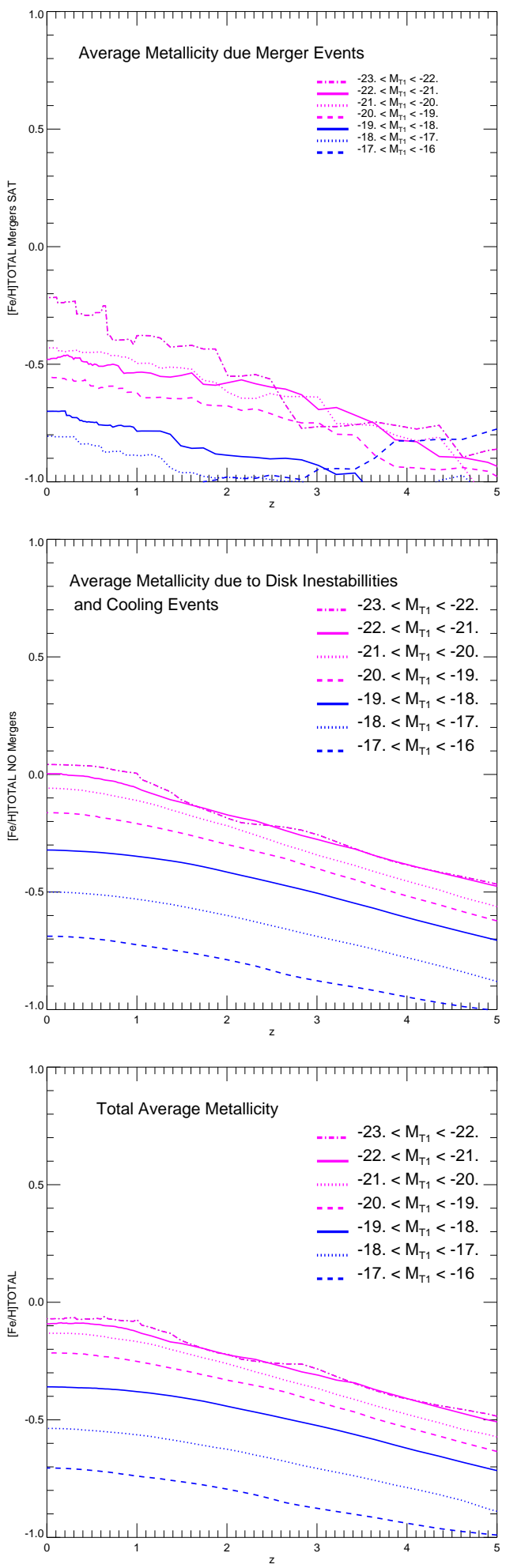

Figura 6.12: Gráficos equivalentes a los de la figura 4.7 para la versión con la nueva formulación de formación estelar: $\mathrm{SAG}_{\mathrm{E} 1+\mathrm{BC} 03+\mathrm{SF} 2}$. Se muestra la evolución con el redshift de metalicidad de la componente estelar de las galaxias de la RCM. Los promedios han sido estimados sobre los cúmulos C15. Panel superior: Metalicidad correspondendiente a la masa estelar acretada y/o generada en fusiones galácticas. Panel medio: Metalicidad debida a la componente estelar generada por los procesos internos de las galaxias. Panel inferior: Metalicidad resultante de la combinación de todos los procesos de formación estelar incluidos en el modelo semianalítico. 


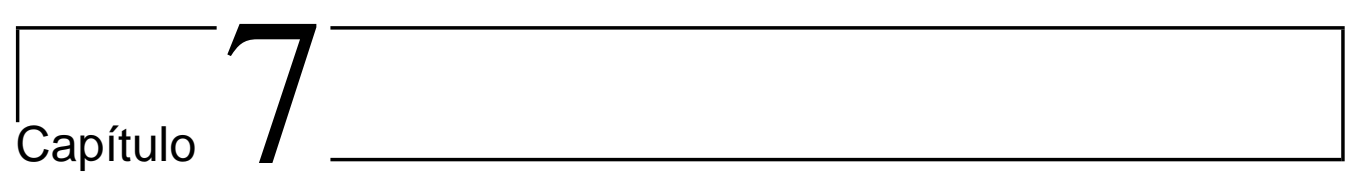

\section{Conclusiones}

La Relación Color Magnitud (RCM) constituye una de las herramientas más importantes para evaluar los modelos de formación de galaxias ya que la evolución de las mismas se evidencia a través de esta relación. Al tratar de entender el modo en que la RCM se desarrolla, y de dilucidar las razones físicas que definen sus características, obtenemos información sobre los procesos evolutivos a los que se ven sometidas las galaxias de distinta masa que pueblan esta relación.

En este Trabajo de Tesis investigamos los procesos físicos que determinan las propiedades de la RCM de cúmulos de galaxias, enfocando nuestra atención en el comportamiento del extremo brillante de esta relación. El estudio se aborda desde un punto de vista teórico, utilizando como herramienta una técnica numérica híbrida que combina simulaciones cosmológicas no radiativas $N$-Body/SPH (Smoothed Particle Hydrodynamics) de cúmulos de galaxias con el modelo semianalítico de formación de galaxias SAG (Cora, 2006; Lagos et al., 2008; Tecce et al., 2010; Jiménez et al., 2011) en el marco cosmológico del modelo de Universo $\Lambda$ CDM. Hemos considerado dos conjuntos de cúmulos simulados con masas en los rangos $\simeq(1,1-1,2) \times 10^{14} h^{-1} \mathrm{M}_{\odot}($ cúmulos $\mathrm{C} 14) \mathrm{y} \simeq(1,3-2,3) \times 10^{15} h^{-1} \mathrm{M}_{\odot}$ (cúmulos C15).

Con el fin de definir el modo más adecuado para identificar las galaxias pertenecientes a la RCM, analizamos distintos criterios de selección existentes en la literatura. Por un lado, estudiamos un criterio basado en la bimodalidad de la distribución de los colores en el diagrama color magnitud de las galaxias, propuesto por Bell et al. (2004). Estudiamos también la RCM resultante de la aplicación de un criterio que tiene en cuenta la morfología de las galaxias, propuesto por Hubble-Jeans. Por último, seleccionamos galaxias pasivas según la tasa de formación estelar específica (Weinmann et al., 2006). Del análisis de la RCM según los diferentes criterios de selección, podemos concluir que, independientemente del criterio utilizado, la RCM muestra características comunes en todos los casos. Es posible distinguir un apartamiento de las galaxias más brillantes de la RCM con respecto a la tendencia mostrada por las galaxias más débiles. Este quiebre ocurre aproximadamente en la misma magnitud en distintas bandas fotométricas, esto es, $M_{R}^{\text {quiebre }} \sim M_{V}^{\text {quiebre }} \sim M_{T 1}^{\text {quiebre }} \approx-20$, que corresponde a una masa media de $1 \times 10^{10} M_{\odot}$. Las galaxias más brillantes que esta magnitud de quiebre presentan un color casi constante $\left(C-T_{1} \approx 1,5\right)$, y la masa media de las galaxias con la magnitud del quiebre es $\sim 10^{10} M_{\odot}$. Este comportamiento del extremo brillante de la RCM simulada está en acuerdo con el ajuste no lineal de la RCM que se reporta en diferentes observaciones (Baldry et al., 2006; Janz \& Lisker, 2009; Baldry et al., 2006; Skelton et al., 2009). El entendimiento de las razones que provocan el apartamiento del extremo brillante de la RCM con respecto a la tendencia marcada por el ajuste lineal a toda la relación constituyó la principal motivación de este Trabajo de Tesis.

La intersección de los tres criterios de selección vistos en el capítulo 3 tiene como factor común galaxias rojas, con poca formación estelar y dominada por bulbos que 
pueblan el extremo brillante de la RCM. Por ello, indistintamente del criterio utilizado, encontramos que la selección de galaxias engloba las propiedades que distinguen a una población prototípicamente de tipo temprano, lo que representa un aval para poder aplicar las conclusiones que se desprenden de esta investigación a las Early Type Galaxies (ETGs). Dado que, en las RCM simuladas, el quiebre en el extremo brillante emerge independientemente del criterio usado para seleccionar galaxias, se adoptó el criterio de selección propuesto por Bell et al. (2004) para llevar a cabo el análisis restante en este trabajo. Este criterio presenta la ventaja de depender del redshift, lo que resulta de gran utilidad para seleccionar galaxias a más alto redshift de manera simple sin hacer uso de hipótesis ad hoc, permitiendo extender a futuro la investigación desarrollada en este trabajo. Una de las relaciones observacionales con las que las galaxias simuladas muestran un muy buen acuerdo es la relación luminosidad metalicidad. Este resultado sustenta el uso de la historia química de las galaxias simuladas como una herramienta para facilitar el entendimiento del desarrollo de la RCM y de su quiebre en el extremo brillante.

Con este objetivo, nos enfocamos en el análisis de la masa de gas, estrellas y metales aportados a cada galaxia por los diferentes procesos físicos involucrados en su evolución, esto es, la formación estelar en modo pasivo y los brotes de formación estelar durante las inestabilidades de disco y las fusiones galácticas. Estudiamos las propiedades medias de las galaxias en siete intervalos de magnitud diferentes, donde las galaxias son agrupadas de acuerdo con su valor de $M_{T_{1}}$ (en el sistema fotométrico de Washington) en $z=$ 0. A partir del análisis de las masas estelares acumuladas aportadas por los diferentes procesos evolutivos, hemos obtenido información sobre la importancia relativa de los mismos evolucionando con el redshift, para galaxias en un dado intervalo de magnitudes. También se obtiene información sobre la relevancia de estos procesos según la luminosidad de las galaxias. A continuación, se puntualizan los principales resultados hallados respecto a la relevancia de los distintos procesos físicos que determinan las propiedades de las galaxias de la RCM; la interpretación de los mismos nos permite arribar a las conclusiones finales de este Trabajo de Tesis, referentes al entendimiento del quiebre del extremo brillante de la RCM. A saber:

- La formación estelar en modo pasivo aparece como el proceso dominante que contribuye a la masa estelar de las galaxias, en todos los intervalos de magnitud y a todo redshift. La fracción de masa aportada por este proceso disminuye monótonamente con el redshift (decreciente) a medida que el reservorio de gas frío de las galaxias se va agotadando (capítulo 4 ).

- Las galaxias con bajas luminosidades $\left(-18<M_{T 1} \leq-17\right.$, intervalo 6$)$ reciben un aporte importante de masa estelar formada durante las fusiones húmedas mayores y menores. Las primeras dan lugar a una mayor fracción de masa que aumenta con el redshift y alcanza valores de $\approx 25$ por ciento a $z \approx 2$. Esto indica que las fusiones húmedas menores no juegan un rol importante a partir de esa época.

- Las galaxias más luminosas $\left(-24<M_{T 1} \leq-22\right.$, intervalo 1$)$ poseen una contribución considerable de fusiones secas menores, la cuales se vuelven importantes a partir de $z \approx 6$. La fracción de masa correspondiente alcanza un valor de $\approx 22$ por ciento a $z=0$. La contribución de las fusions secas mayores es también relevante para estas galaxias a partir de $z \approx 2$, llegando a contribuir con una fracción de masa de $\approx 10$ por ciento a $z=0$.

- La transición entre la importancia relativa de las contribuciones de las fusiones húmedas y secas ocurre para las galaxias con magnitudes en el rango $-21<M_{T 1} \leq$ -20 (intervalo 3), i.e. para las galaxias con magnitudes en el intervalo donde se produce el quiebre hacia colores más azules de la RCM. La masa promedio de éstas galaxias es $\sim M_{T_{1}}^{\text {quiebre }} \approx-20$. En esta transición, las fusiones secas menores empiezan a ganar importancia, mientras que la relevancia de las estrellas formadas durante los brotes estelares asociados a las fusiones húmedas menores decrece considerablemente a partir de $z \approx 4$, volviéndose despreciable a $z=0$. Esto está en 
fuerte contraste con lo observado para este mismo proceso en las galaxias menos luminosas (intervalo 6). Encontramos, también, una contribución moderada de las fusiones húmedas mayores a bajos redshifts. Todos estos eventos de fusiones galácticas son responsables del $\approx 20$ por ciento de la masa total recibida por estas galaxias.

A partir del análisis de la importancia relativa de las fracciones de masa estelar aportadas por los diferentes procesos, es claro que la evolución de las galaxias en el extremo brillante de la RCM está dominada por la contribución seca de las fusiones tanto mayores como menores, mientras que en las galaxias más débiles la evolución está influenciada por la componente húmeda de las fusiones. Esta situación queda establecida ya a $z \approx 1$. Las metalicidades de las galaxias en el extremo brillante no cambian a partir de ese momento (redshift), mientras que un aumento moderado de la metalicidad es detectado para las galaxias menos luminosas, donde sigue teniendo lugar la formación estelar a partir de gas enriquecido en metales (capítulos 4,5).

Considerando la masa acumulada desde $z=1$, tanto de las estrellas formadas in situ, como de la masa estelar que ya estaba presente en las galaxias satélites acretadas, encontramos que la última representa una fracción importante de la masa estelar actual de las galaxias en el extremo brillante de la RCM, con valores que oscilan entre el 10 y el 20 por ciento. Esta fracción es mayor que la fracción de estrellas generadas debido a la formación estelar reciente. Si bien la metalicidad de las estrellas formadas desde $z \approx 1$ es alta $(\approx 0,2-0,5$ dex mayor que la abundancia de hierro promedio en las galaxias a $z=0)$, la masa aportada por estas estrellas es tan baja $(\approx 0,05)$ que no afecta la metalicidad media de las galaxias. Por el contrario, las fusiones secas proveen una fracción más alta de masa estelar que la aportada por la formación estelar reciente, pero con un contenido bajo de metales $(\approx 0,2$ dex menor que la metalicidad media de las galaxias). En consecuencia, las fusiones secas contribuyen al aumento de la masa estelar sin alterar su metalicidad promedio. Por lo tanto, las galaxias en el extremo brillante, las cuales poseen muy poca actividad de formación estelar a partir de $z \approx 1$, alcanzan un límite superior en sus abundancias químicas. Teniendo en cuenta la pequeña dispersión en la edad de estos sistemas, al fijarse el contenido de metales de estas galaxias, se fijan también sus colores. El apartamiento del extremo brillante de la RCM con respecto a la tendencia lineal observada a menores luminosidades puede, entonces, ser explicado por el efecto de la contribución de las fusiones mayores y menores secas, las cuáles aumentan la masa/luminosidad de estas galaxias sin alterar sus colores significativamente.

Para testear la robustez de nuestras conclusiones sobre la explicación del comportamiento del extremo brillante de la RCM, estudiamos el impacto sobre las propiedades de la RCM de nuevas implementaciones incluidas en el modelo semianalítico SAG, que comprenden: el efecto de la extinción por polvo, la inclusión de la emisión de estrellas en la fase TP-AGB, y una formulación alternativa para describir el proceso de formación estelar. Estas nuevas implementaciones alteran los colores, las edades y las metalicidades de las galaxias que pueblan la RCM. Analizamos la influencia sobre la RCM de cada implementación por separado, y luego, el efecto de la acción simultánea de todas ellas, manteniendo siempre los parámetros de calibración de SAG utilizados en todo el análisis previo. Encontramos variaciones en la dispersión de la RCM según la modificación aplicada, siendo éstas más significativas por la influencia del nuevo modelo de extinción por polvo. Cabe destacar que el quiebre en el extremo brillante de la RCM se mantiene presente cuando aplicamos las nuevas implementaciones, tanto en forma separada como combinada (capítulo 6).

Gran parte de estas conclusiones han sido volcadas en el trabajo de Jiménez et al. (2011), y son válidas para las galaxias pertenecientes a cúmulos de alta y baja masa. Hemos demostrado que las propiedades locales de las galaxias parecen haber sido establecidas alrededor de $z \approx 1$, en acuerdo con resultados de estudios observacionales (Strateva et al., 2001; Blanton et al., 2003; Baldry et al., 2004, 2006; Menci et al., 2008; Mei et al., 2009; Eliche-Moral et al., 2010). Un análisis detallado de los rasgos de la RCM, 
como su dependencia con el ambiente y el redshift, forma parte del trabajo proyectado a futuro. 


\section{Bibliografía}

Allard France \& Hauschildt Peter H., 1995, ApJ, 445, 433A

Asplund M., Grevesse N., Sauval A. J., 2005, in T.G. Barnes III, F.N. Bash, eds, ASP Conf. Ser. Vol. 36, Cosmic Abundances as Records of Stellar Evolution and Nucleosynthesis. Astron. Soc. Pac., San Francisco, p. 25

Avila-Reese, V, 2007, Ap\& SS Proceedings, "Solar, stellar and galactic connections between particle physics and astrophysics", Springer , pp. 115-165

Baldry I. K., Glazebrook K., Brinkmann J., Ivezić Z., Lupton R. H., Nichol R. C., Szalay A. S., 2004, ApJ, 600, 681

Baldry I. K., Balogh M. L., Bower R. G., Glazebrook K., Nichol R. C., Bamford S. P., Budavari T., 2006, MNRAS, 373, 469

Balogh M. L., Navarro J. F., Morris S. L., 2000, ApJ, 540, 113

Baugh C. M., 1996,MNRAS,280,267B

Bekki K., 2009, MNRAS, 399, 2221

Bell F. E., Wolf C., Meisenheimer K., Rix H.-W. et al., 2004, ApJ, 608, 752

Bell F. E., Naab T., McIntosh D. H. et al., 2006, ApJ, 640, 241

Bell F. E., Phleps S., Somerville R., Wolf C., Borch A., Meisenheime K., 2006, ApJ, 652, 270

Bernardi M., Hyde J. B., Sheth R. K., Miller J. C., Nichol R. C., 2007, ApJ, 133, 1741

Bernardi M., Sheth R. K., Nichol R. C., Schneider D. P., Brinkmann J., 2005, AJ, 129, 61

Bernardi M., 2009, MNRAS, 395, 1491

Bernardi M., Roche N., Shankar F., Sheth R. K., 2010, MNRAS, submitted (arXiv:1005.3770)

Bessell M. S., 2001, PASP, 113, 66

Bezanson R., van Dokkum P. G., Tal T., Marchesini D., Kriek M., Franx M., Cpi P., 2009, Apj, 697, 1290

Binney J., \& Tremaine S.,1987, Princeton, NJ, Princeton University Press, 1987, 747 p.

Binney J., \& Tremaine S., 2008, "Galactic Dynamics: Second Edition", Princeton University Press 
Blakeslee J. P., Franx M., Postman M., Rosati P., Holden B., Illingworth G. D., Ford H. C., Cross N. J. G., et al., 2003, ApJL, 596, 143

Blanton M.R., Hogg D.W., Bahcall N.A., Baldry I.K., Brinkmann J., et al., 2003,ApJ,594,186B

Blanton M.R., Schlegel D.J., Strauss M.A., Brinkmann J., Finkbeiner D., et al., 2005 AJ, $129,2562 \mathrm{~B}$

Blommaert J., Groenewegen M., Okumura K., Ganesh, S., Omont A., 2006, A\&A,460

Miller Bertolami M. M., Tesis Doctoral, 2009, Universidad Nacional de La Plata, FCAG

Borgani S., Murante G., Springel V., Diaferio A., Dolag K., et al., 2004,MNRAS, $348,1078 \mathrm{~B}$

Bournaud F., Jog C. J., Combes F., 2007, A\&A, 476, 1179

Bower R., Lucey J., Ellis R., 1992, MNRAS, 254, 601

Bower R. G., Kodama T., Terlevich A., 1998, MNRAS, 299, 1193

Bruzual G., Charlot S., 2003, MNRAS, 344, 1000

Bruzual G., Charlot S., 2003, MNRAS, 344, 1000

Bruzual G., Charlot S., 2003, MNRAS, 344, 1000

Bruzual G., 2007,ASPC,374,303B

Bundy K., Fukugita M., Ellis R. S., Targett T. A., Belli S., Kodama T., 2009, ApJ, 697, 1369

Canterna R., Harris H.C., 1979,DudOR 14,199C

Cappellari Michele; di Serego Alighieri S., Cimatti A., 2009, ApJL, 704, 34

Cardelli, J., A., Clayton G., Mathis, J., 1989 ApJ,345

Cattaneo A., Mamon G. A., Warnick K., Knebe A., 2011, A\&A,533,A,5C

Cenarro A. J., Trujillo I., 2009, ApJL, 696, 43

Charlot S. \& Bruzual G., 2007, Modelos no publicados, cedidos por pedido. (Sofía Cora, comunicacion privada).

Cole S., Aragon-Salamanca A., Frenk C., Navarro J., \& Zepf S.E., 1994, MRAS 271, 781-806

Cole S., Lacey C., Baugh C., Frenk C., 2000, MNRAS,319,168C

Conselice C., 2006, MNRAS, 373, 1389

Conselice C., Gallagher J. S., Wyse R. F., 2003, AJ, 125, 66

Conselice C., Yang C..,Bluck A. F., 2009, MNRAS, 394, 1956

Cora S. A., 2006, MNRAS, 368, 1540

Croton D. J., Springel V., White S. D. M., et al., 2006, MNRAS, 365, 11

Interactive Data Language, David Stern \& ITT Visual Information Solutions (ITT VIS), 1977, Versión IDL 7.1, Licencia para la Universidad Nacional de La Plata. URL:www.exelisvis.com/language/en-US/ProductsServices/IDL.aspx 
de Vaucouleurs G., 1961, ApJS, 5, 233

Daddi E., Renzini A., Pirzkal N., et al., 2005, ApJ, 626, 680

De Lucia G., Kauffmann G., White S., 2004, MNRAS,349,1101D

De Lucia G., Blaizot J., 2007, MNRAS, 375, 2

De Propris R., Colless M., Driver S. P., Couch W., Peacock J. A., Baldry I., Baugh C. M., Bland-Hawthorn J., et al., 2003, MNRAS, 342, 725

de Rijcke S., Penny S. J., Conselice C. J., Valcke S., Held E. V., 2009, MNRAS, 393, 798

Devriendt J.E.Gn., Guiderdoni B., Sadat, R., 1999, A\&A, 350, 381

Demers S., Battinelli P., Letarte B.,2004, A\&A, 424, 125D

Dirsch B., Richtler T., Bassino L.P., 2003, A\&A, 408, 929

Dolag K., Vazza F., Brunetti G., Tormen G., 2005, MNRAS, 364, 753

Dunkley J., Komatsu E., Nolta M., Spergel D., Larson D., et al, 2009, APJSS, 180, 306-329

Eliche-Moral M., Prieto M., Gallego J., Zamorano J., 2010, ApJ, submitted (arXiv:1003.0686)

Fan L., Lapi A., Bressan A., Bernardi M., De Zotti G., Danese L., 2010, ApJ, 718, 1460

Fakhouri O., Ma C.-P., Boylan-Kolchin M., 2010, MNRAS, 406, 2267

Ferrarese L., Coté P., Jordan A. et al., 2006, ApJS, 164, 334

Merritt D., \& Ferrarese L.,2001,MNRAS, 320, L30-L34

Font A. S. et al., 2008, MNRAS, 389, 1619

Fontanot Fabio, Monaco Pierluigi, 2010, MNRAS,405,705F

Forbes D., Forte J. C., 2001, MNRAS, 322, 257

Forestini M., Arnould M., Paulus G., 1991, A\&A, 252, 597F

Forte J. C., Faifer F. R., Geisler D., 2007, MNRAS, 382, 1947

Fukugita M., Shimasaku K., Ichikawa T., 1995, PASP, 107, 945

Gallagher J., Hunter D., Bushouse H., 1989, AJ, 97, 700

Gallazzi A., Charlot S., Brinchmann J., White S. D. M., 2006, MNRAS, 370, 1106

Geisler D., 1996, AJ, 111, 480

Gerke B.F., Newman J. A., Faber S. M., Cooper M. C. et al. 2007, MNRAS, 376, 1425G

Grebel E. K., Gallagher J. S. III, Harbeck D., 2003, AJ, 125, 1926

Grebel E., Seitzer P., Dolphin A., Geisler D., Guhathakurta P., et al., 1999, AAS, 195, $0803 \mathrm{G}$

Guiderdoni B. \& Rocca-Volmerange B., 1987, A\&A, 186, 1

Guo, Qi, White, Simon D. M., 2009, MNRAS, 396, 39G

Haines C. P., Gargiulo A., La Barbera F., Mercurio A., Merluzzi P., Busarello G., 2007, MNRAS,381,7H 
Haring N., \& Rix H.W.. 2004, ApJL, 604,L89-L92

Harris W. E., Harris G. L., 2002, AJ, 123, 3108

Hatton S., Devriendt J., Ninin S., Bouchet F., Guiderdoni B., et al., 2003, MNRAS, $343,75 \mathrm{H}$

Hernquist, L. , Mihos, J. C. , 1995, ApJ , 448, 41H

Henriques B., Maraston C., Monaco P., Fontanot F., Menci N., et al. 2011, MNRAS, 415,3571

Hilker M., Mieske S., Infante L., 2003, A\&A, 397, L9

Hogg, D. W., et al. 2004, ApJL, 601, L29

Hopkins P. F., Hernquist L., Cox T. J., Keres D., Wuyts S., 2009, ApJ, 691, 1424

Hopkins P. F., Croton D., Bundy K., Khochfar S., van den Bosch F., et al. 2010, ApJ,724,915H

Hopkins P. F., Quataert E., Murray N., 2011,MNRAS,417,950H

Iwamoto K., Brachwitz F., Nomoto K., Kishimoto N., Umeda H., et al. 1999, APJSS, $125,439-462$

Jaff, Y. L., Aragon-Salamanca A., De Lucia G., Jablonka P., Rudnick G., Saglia R., Zaritsky D., 2010, MNRAS, in press (arXiv:1007.1425)

Jansen R., Fabricant D., Franx M., Caldwell N., 2000, ApJS,126,331J

Janz J., Lisker T., 2009, ApJ, 696, L102

Janz J., Lisker T., 2008, ApJ, 689, L25

Jiménez N., Cora S. A., Bassino L. P., Tecce T. E., Smith Castelli A. V., 2011, MNRAS, 417,785

Jogee S., Miller S. H. , Penner K. , et al., 2009, Apj, 697, 1971

Karick A. M., Drinkwater M. J., Gregg M., 2003, MNRAS, 344, 188

Kauffmann G., White S., \& Guiderdoni B., 1993,MNRAS 264, 201-218

Kauffmann G., Colberg J. M., Diaferio A., White S. D. M., 1999, MNRAS, 303, 188

Kaviraj S., Devriendt J. E. G., Ferreras I., Yi S. K., 2005, MNRAS, 360, 60

Kaviraj S., Schawinski K., Devriendt J. E. G., Ferreras I., Khochfar S., Yoon S.-J., Yi S. K., Deharveng J.-M., et al., 2007, APJS, 173, 619

Kaviraj S., Khochfar S., Schawinski K., Yi S. K., Gawiser E., et al., 2008, MNRAS, 388, 67

Kaviraj S., Tan K.-M., Ellis R. S., Silk J., 2011, MNRAS, 411, 2148

Kawata D., Mulchaey J. S., 2008, ApJ, 672, L103

Kennicutt Robert C. Jr., 1998, ApJ,498,541K

Khochfar S., Burkert A., 2003, ApJL, 597, 117

Khochfar S., Silk J., 2006, ApJ, 648, L21 
Kimm T., Somerville R. S., Yi S.K., van den Bosch F., Salim S., Fontanot F., Monaco P., Mo H., et al., 2009, MNRAS,394,1131K

Kimm T., Yi S. K., Khochfar S., 2011,ApJ,729,11K

Kodama T., Bower R., 2001, MNRAS, 321, 18

Kurucz, R. L., 1992,RMxAA,23,45K

Lagos C., Cora S. A., Padilla N. D., 2008, MNRAS, 388, 587

Lagos C., Padilla N. D., Cora S. A., 2009, MNRAS, 397, L31

Lanzoni B., Guiderdoni B., Mamon G. A., Devriendt J., Hatton S., 2005, MNRAS, 361, 369

Larson R. B., Tinsley B. M., Cadwell C. N., 1980, ApJ, 237, 692

Lin L., Cooper M. C., Jian H.-Y. et al., 2010, ApJ, 718, 1158

Lisker T., Grebel E. K., Binggeli B., 2008, AJ, 135, 380

Lopez-Sanjuan,C., Le Favre, O., de Ravel, L., Cucciati, O., Ilbert, O., Tresse, L., et al. 2010, accepted for publication in A\&A

Liu F. S., Mao S., Deng Z. G., Xia X. Y., Wen Z. L., 2010, MNRAS, 396, 2003

López-Cruz O., Barkhouse W. A., Yee H. K. C., 2004, ApJ, 614, 679

Maiolino, R., Schneider, R., Oliva, E., Bianchi, S., Ferrara, A., Mannucci, F., Pedani, M., Roca Sogorb, M., 2004, Natur, 431, 533M

Malbon R. K., Baugh C. M., Frenk C. S., Lacey C. G., 2007, MNRAS, 382, 1394

Mancini C., Daddi E., Renzini A., Salmi F., McCracken H. J., Cimatti A., Onodera M., Salvato M., et al., 2010, MNRAS, 401, 933

Maraston C.,2005,MNRAS,362,799

Maraston C., Daddi E., Renzini A., Cimatti A., Dickinson M., et al., 2006, ApJ,652,85M

Marigo P., Girardi L, 2007, A\&A, 469, 239M

Marigo P., 2001, A\&A,370,194-217

Marconi A., \& Hunt L., 2003, ApJ 589,L21-L24,

Martinez H. J., Coenda V., Muriel H., MNRAS, 403, 748

McCarthy I. G. et al., 2008, MNRAS, 383, 593

McIntosh D. H., Guo Y., Hertzberg J., Katz N., Mo H. J., van den Bosch F. C., Yang X., 2008, MNRAS, 388, 1537

McIntosh D., Rix H.W., Caldwell N., 2004,ApJ,610,161M

McIntosh D.H., Zabludoff A.I., Rix H.W., Caldwell N., 2005,ApJ,619,193M

Mei S., Holden B., Blakeslee J. P., Ford H. C., Franx M., Homeier N. L., Illingworth G. D., Jee M. J., et al., 2009, APJ, 690, 42

Mei S., Holden B. P., Blakeslee J. P., Rosati P., Postman M., Jee M. J., Rettura A., Sirianni M., et al., 2006, ApJ, 644, 759 
Menci N., Rosati P., Gobat R., Strazzullo V., Rettura A., Mei S., Demarco R., 2008, ApJ, 685, 863

Mendel J. T., Proctor R. N., Rasmussen J., Brough S., Forbes D. A., 2009, 396, 2103

Mieske S., Hilker M., Infante L., Mendes de Oliveira C., 2007, A\&A, 463, 503

Misgeld I., Mieske S., Hilker M., 2008, A\&A, 486, 697

Misgeld I., Hilker M., Mieske S., 2009, A\&A, 496, 683

Mowlavi N. \& Meynet G., 2000, A\&A, 361, 959M

Mo H., Mao S., White S., 1998, MNRAS, 295, 319M

Mo H., Van den Bosch F.\& White S., 2010, Cambridge University Press, New York, USA.

Moore B., Lake G., Quinn T., Stadel J., 1999, MNRAS, 304, 465

Naab T., Johansson P. H., Ostriker J. P., Efstathiou G., 2007, ApJ, 658, 710

Naab T., Johansson P. H., Ostriker J. P., 2009, ApJ, 699, L178

Navarro J.,\& White S.,1994,MNRAS,267,401N

Nakazawa K., Makishima K., Fukazawa Y., Tamura T., 2000, PASJ, 52 ,623

Nierenberg, A. M.; Auger, M. W.; Treu, T.; Marshall, P. J.; Fassnacht, C. D., 2011,ApJ, $731,44 \mathrm{~N}$

Padovani P., \& Matteucci F., 1993, ApJ, 416,26

Parry O. H., Eke V. R., Frenk C. S., 2009, MNRAS,396,1972P

Pietrinferni A., Cassisi S., Salaris M., Castelli F., 2004 ApJ,612,168P

Pedersen K., Yoshii Y., Sommer-Larsen J., 1997, ApJ, 485, L1

Press W.,\& Schechter P., 1974,ApJ 187, 425-438

Prescott M., Baldry I.K., James P.A., Bamford S.P., Bland-Hawthorn J., et al. 2011, MNRAS, 417,1374P

Portinari L., Chioisi C., Bressan A., A\&A,26A,334,505P

Quadri N., Otro N., NN., 2008, MNRAS, 456,89 COMPLETAR

Reda F., Forbes D., Beasley M., O’Sullivan E., Goudfrooij P., 2004, MNRAS, 354, 851

Reda F., Forbes D., Hau G., 2005, MNRAS, 360, 693

Robaina A. R., Bell E. F., van der Wel A., Somerville R. S., Skelton R. E., McIntosh D. H., Meisenheimer K., Wolf C., 2010, ApJ, 719, 844

Roediger E., 2009, AN, 330, 888

Romeo A. D., Napolitano N. R., Covone G., Sommer-Larsen J., Antonuccio-Delogu V., Capaccioli M., 2008, MNRAS, 389, 13

Ruszkowski M., Springel V., 2009, ApJ, 696, 1094

Sandage A., Visvanathan N., 1978, ApJ, 223, 70 
Saro A., Borgani S., Tornatore L., Dolag K., Murante G., Biviano A., Calura F., Charlot S., 2006, MNRAS, 373, 397

Secker J., Harris W. E., Plummer J. D., 1997, PASP, 109, 1377

Schweizer F.,\& Seitzer P., 1992, AJ,104,1039S

Simien F., de Vaucouleurs G., 1986,ApJ,302,564S

Skelton R., Bell E., Somerville R., 2009 , ApJ, 699, L9

Smith Castelli A. V., Bassino L. P., Richtler T., Cellone S., Aruta C., Infante L., 2008, MNRAS, 386, 2311

Sofue Y., 1994, ApJ, 423, 207

Somerville R., \& Primack J., 1999, MNRAS,310, 1087-1110

Somerville R.S., Hopkins P. F., Cox T. J., Robertson B. E., Hernquist L., 2008, MNRAS, 391,481

Spergel, D. N. et al., 2003, First-Year WilkinsonMicrowave Anisotropy Probe (WMAP) Observations: Determination of Cosmological Parameters. ApJ, Suppl. 148, 175-194

Springel V., White S., Tormen G., Kauffmann G., 2001, MNRAS, 328, 726

Springel V., 2005, MNRAS, 364, 1105

Springel V., White S. D. M., Jenkins A. et al., 2005, Natur, 435, 629

Spolaor M., Proctor R. N., Forbes D. A., Couch W. J., 2009, ApJL, 691, 138

Stott J. P., Pimbblet K. A., Edge A. C., Smith G. P., Wardlow J. L., 2009, MNRAS,394, 2098

Strateva I., Ivezic Z., Knapp G.R., Narayanan V.K., Strauss M.A., et al., 2001 ,AJ, 122 , $1861 \mathrm{~S}$

Sutherland R., Dopita M., 1993, ApJS, 88, 253

Tecce T. E., Cora S. A., Tissera P. B., Abadi M. G., Lagos C. del P., 2010, MNRAS, 408, 2008

Terlevich A., Caldwell N., Bower R., 2001, MNRAS,326,1547T

Tonini C., Maraston C., Thomas D., Devriendt J., Silk J., 2010, MNRAS, 403, 1749T

Tonini C., Maraston C., Devriendt J., Thomas D., Silk J.,2009, MNRAS,396L,36T

Tormen G., Bouchet F., White S. D. M., 1997, MNRAS, 286, 865

Trager S. C., Faber S. M., Worthey G., Gonzalez J. J., 2000a, AJ, 120, 165

Trager S. C., Faber S. M., Dressler A., 2008, MNRAS, 386, 715

van der Wel A., Bell E. F., van den Bosch F. C., Gallazzi A., Rix H.-W., 2009, ApJ, 698, 1232

Visvanathan N., Sandage A., 1977, ApJ, 216, 214

Whitaker K. E., van Dokkum P. G., 2008, ApJL, 676, 105

van Dokkum P. G., Whitaker K. E., Brammer G., Franx M., Kriek M., Labbe I., Marchesini D., Quadri R., et al., 2010, Apj, 709, 1018 
Wang, B., \& Heckman, T., 1996, ApJ, 457, 645

Weinmann S. M., van den Bosch F. C., Yang X., Mo H. J., 2006, MNRAS, 366, 2

Whitaker K. E., van Dokkum P. G., 2008, ApJL, 676, 105

White S., \& Frenk C., 1991, Apj,379,52-79

Woosley S.E., Weaver Thomas A, 1995,ApJS,101,181W

York D., Adelman J., Anderson J.E., Jr. Anderson S.F., 2000, AJ,120,579Y

Yoshida N., Sheth R. K., Diaferio A., 2001, MNRAS, 328, 669 


\subsection{Agradecimientos}

El trabajo de volverse una astrónoma profesional lleva, en la Universidad Nacional de La Plata, teóricamente 5 años de carrera, que en mi caso fueron un par más, sumados a los 5 años de doctorado. Los plazos son establecidos también por CONICET, quien solventó mi formación de doctorado con dos becas sucesivas. A estas dos instituciones les agradezco por la formación y el apoyo recibidos. De la misma manera, quedo eternamente agradecida a todo el pueblo de la Nación Argentina, que mantiene un sistema único en el mundo de educación (y salud) de calidad y gratuita. Este sistema fué el que permitió que mis abuelos recién llegados de una agonizante España franquista, pudieran aún trabajando de verduleros, tener un hijo egresado de la Universidad Nacional de Salta. Mi mamá por su parte, también egresada de la misma Universidad, accedió a estudios superiores que de haber sido pagos hubieran resultado prohibitivos para una familia numerosa, donde el único profesional era mi abuelo médico boliviano. La movilidad social que aún hoy genera el paso por la Universidad hacen de Argentina un gran país. Gracias al esfuerzo y estudio de mis padres, accedí yo a una Universidad aún más grande y más prestigiosa.

En mi camino por los claustros tuve siempre el desafío de sobrevivir, aprender y hacer de esto un camino gustoso, al fin. En los primeros años la ayuda y la dedicación de Juan Racker, excelente ayudante de cuánta materia le asignaran, hicieron mella en la forma de encarar los problemas y aplicar el método científico. A Juan, por la paciencia, la guía y la amistad, muchas gracias.

Los amigos de aquellos primeros años, muchos ya fuera de la Universidad o el sistema científico recordarán conmigo las interminables tardes/noches de mates y ecuaciones. Muchas gracias a Angeles Moliné y a toda su familia por haberme adoptado, prácticamente. Hicieron de La Plata un hogar en los primeros años. A la salteña Natalia Galván, a Anabella Araudo, Mariana Orellana, Hernán Lugea, Laura Suad, Cecilia Garrafo, Natalia Rahmer y todo el grupo que de chicos con los que cursé hasta tercer año. Durante el segundo año tuve la suerte de conocer a dos de los aún hoy son mis mejores amigos; Soledad Montenegro y (por intermedio de Demian Slobinsky), a mi querido Agustín García. Con ellos estudiamos y discutimos muchas de las materias en común de las tres carreras (Geofísica, Matemática y Astronomía) y otros muchos temas.

Mi tesis de licenciatura fue realizada bajo la dirección del Profesor Angel L. Plastino, a quien agradezco la (inmerecida) confianza que depositó en mi en aquellos primeros pasos en la investigacion. Supo ver más allá de mis inseguridades y augürarme un buen camino en la Ciencia. Muchas gracias por la generosidad en los elogios, la confianza y el respeto a las mujeres, que luego vi, son un bien escaso en el mundo (también académico). La Tesis de Licenciatura está dedicada a mi querida hermana Rocío y a Soledad Montenego, con quienes vivimos muchos años felices en La Plata.

Durante los años de doctorado nada hubiese podido hacerse sin el apoyo y el amor de quien fuera mi pareja, Marcelo Miller Bertolami. Lo sabe y lo sé. Gracias por toda la ayuda, comentarios, dicusiones, y cariño que rodearon este proceso.

Mis nuevos amigos del fondo le dieron nuevo color a cada día. Muchas gracias al Dr. Cascotes (Gonzalo De Elia), doña Ileana Adruchow (extensivo a esbozo Alejandro), Mariana Orellana y Anabella Araudo. A las chicas de la oficina, Lucila Kraiselburg, Mercedes Mosquera, Claudia Sccocola, especialmente a Florencia Anabella Teppa Pania (el alma de la fiesta). A Alejando Córsico, Leandro Althaus y Jorge Panei por discusiones y las risotadas que traspasaban paredes. Un cálido reconocimiento a mis compañeros de doctorado, entre todos todo era más divertido: Nicolás Maffione, Martín Mestre, Aleandra Romero, Octavio Guilera, Ignacio Gargiulo y a Tomás Tecce del IAFE. A Juan Pablo Caso y a mis compañeros de la cátedra de "Estadística Aplicada". También quiero destacar la generosidad, calidez y excelencia del Profesor Hector Vucetich y Susana Landau, con quienes gané la Beca Tipo I de CONICET, que permitió el comienzo del doctorado.

Agradecimientos también a la IAU por la ayuda económica muchas veces recibida, en especial por la que permitió mi participation en el IAU Symposium 284, en la Universidad of Central Lancashire (Inglaterra), donde pude discutir los resultados principales de mi 
tesis de doctorado con colegas especializados en el tema. Muchas gracias a la asociación Max Planck Institute fur Astronomy de Alemania, ya con base en la Universidad de Heidelberg, donde concurrí a la escuela de verano del año 2010, ya en Garching, Munich, por recibirme como visitante en el 2009.

Un reconocimiento especial a Patricia Tissera y todo el grupo de Astrofísica Numérica del IAFE, quienes organizan Workshops cada dos años y atraen al país a los más prestigiosos astrónomos de todo el mundo. De igual manera, a Nelson Padilla y a todo el grupo de la Universidad Católica de Chile, con quienes colaboramos cercanamente. Quedo en deuda con el Profesor Diego García Lambas, del Observatorio de Córdoba, quien accedió a dictar de manera no convencional el curso "Estructura a Gran Escala en el Universo" y además solventó parte de los gastos que hicieron posible los viajes al Observatorio de Córdoba. Mi reconocimiento para un gran profesor y una gran persona.

Las actividades fuera del Observatorio fueron un privilegio que ofrece la ciudad de La Plata y un placer necesario. El paso por el Conservatorio Gilardo Gilardi y los claustos de la Facultad de Humanidades, donde cursé parte de la carrera de Historia, fueron fundamentales para mi formación en su sentido más amplio. De la misma manera, gracias a los talleres de la Municipalidad de La Plata descubrí un mundo nuevo en las Danzas Clásicas. Muchas gracias a mis maestros: Raquel Rizzo (por su excelencia y sentido del humor), Sol Poli, Marisa Fontanta y a mi querido Juan Esteban Irigoyen. Junto con las chicas del Taller de Danzas de la UNLP hicimos de "Giselle" la coreografía más soñada. A mis amigas bailarinas, Laura Baez y Guillermina Gulo Tieri. En el plano musical, muchas gracias a Andrés Bugallo y a su gran familia. A toda la gente del Coro de La Catedral de La Plata, por los miles de los momentos compartidos y el disfrute de cantar juntos partituras de Brahms, Mozart, Bach, Mendelssohn y muchos más... sin todo este paquete artístico no hubiera podido terminar mis estudios y mi trabajo, en la aridéz del desierto exacto. No puedo olvidar la incondicionalidad de Vicky-Vicuña (Cuñita) y Cañita-Macchiata que estuvieron siempre y aún más cuando escribía esta tesis a altas horas de la madrugada. Así mismo no puedo dejar de agradecer a Gabriela. Y y a mi hermanos Santiago y Ezequiel.

Seguramente me estoy olvidando de mucha gente de la Universidad y CONICET y todos los organismos estatales, quienes con su trabajo de cada día posibilitaron mi camino y la llegada a buen puerto. A ellos, mi reconocimiento. 